\title{
MICROSCOPIC ANALYSIS OF
}

\section{HIGH FORCHHEIMER NUMBER FLOW IN POROUS MEDIA}

by

Huiping Ma

\author{
A Thesis \\ Submitted to the Faculty of Graduate Studies \\ in Partial Fulfillment of the Requirement \\ for the Degree of
} DOCTOR OF PHILOSOPHY

Department of Mechanical and Industrial Engineering

University of Manitoba

Winnipeg, Manitoba, R3T 2N2 Canada

(C) October, 1993 
Acquisitions and

Bibliographic Services Branch

395 Wellington Street

Ottawa. Ontario

K1A ON4
Bibliothèque nationale

du Canada

Direction des acquisitions et

des services bibliographiques

395, rue Wellington

Ottawa (Ontario)
Yourlite votre relèrence

Our hile Nolre rêtérence
The author has granted an irrevocable non-exclusive licence allowing the National Library of Canada to reproduce, loan, distribute or sell copies of his/her thesis by any means and in any form or format, making this thesis available to interested persons.
L'auteur a accordé une licence irrévocable et non exclusive permettant à la Bibliothèque nationale du Canada de reproduire, prêter, distribuer ou vendre des copies de sa thèse de quelque manière et sous quelque forme que ce soit pour mettre des exemplaires de cette thèse à la disposition des personnes intéressées.
The author retains ownership of the copyright in his/her thesis. Neither the thesis nor substantial extracts from it may be printed or otherwise reproduced without his/her permission.
L'auteur conserve la propriété du droit d'auteur qui protège sa thèse. Ni la thèse ni des extraits substantiels de celle-ci ne doivent être imprimés ou autrement reproduits sans son autorisation.

ISBN $\quad 0-315-92279-6$ 
Name HUIPING MA

Dissertation Abstracts International is arranged by broad, general subject categories. Please select the one subject which most nearly describes the content of your dissertation. Enter the corresponding four-digit code in the spaces provided.

Engineering, Mechamical
\begin{tabular}{|l|l|l|l|}
\hline 0 & 5 & 4 & 8 \\
\hline
\end{tabular} U.M.M.I SUBJECT CODE

\section{Subject Categories}

\section{THE HUMANITIES AND SOCIAL SCIENCES}

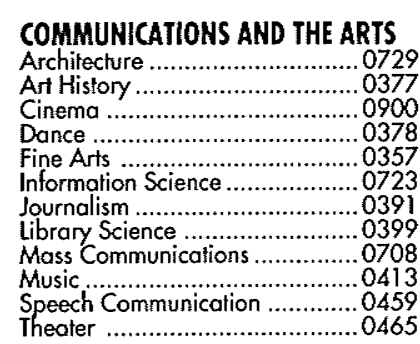

\section{EDUCATION}

General ..................................0515

Administration ...........................0514

Adult and Continuing …................0516 Agricultural ..............................0517

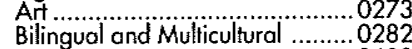
Business ................................068 Community College ...................0275 Earriculy Childhood and ........................0518 Elementary ..................................0524 Finance ....................................... Guidance and Counseling ….......0579 Higher .........................................0745 Hisłory of .............................

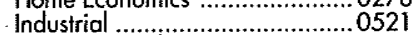
Language and Literature ................0279 Language and tilerature .............0280

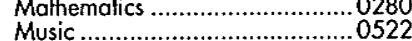

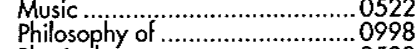
Physical ......................................0523
Psychology ………...................0525

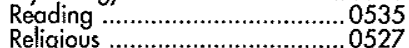

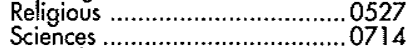
Secondary ....................................0533 Social Sciences ...........................0534 Sociology of .............................0340 Teacher Training .......................... 0530 Teacher Training .......................0530 Technology .............................0710
Tests and Measurements ...........0288 Vocational ..................................0747

\section{LANGUAGE, LITERATURE AND} LINGUISTICS

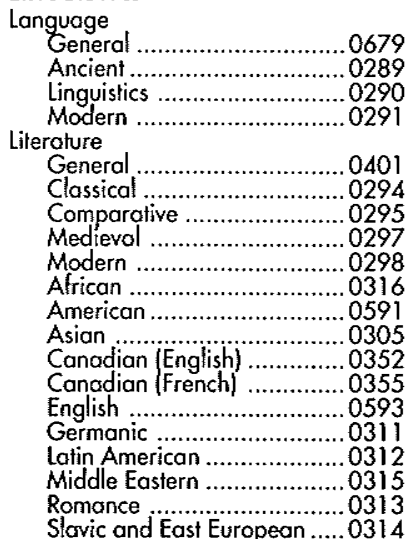

PHILOSOPHY, RELIGION AND THEOLOGY

Philosophy .................................0422

Religion

General ..............................0318

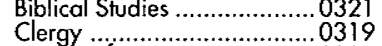

Philosophy of ....................... 0322

SOCIAL SCIENCES

Americon Studies ......................0323

Anthropology

Cullural ...................................0326

Business Administration

General .............................0310

Accounting …....................0272

Banking ….........................0770

Monagement ......................0454

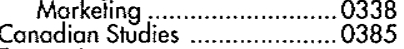

Economics

General .............................0501

Agricultural ............................0503

Commerce-Business ............0505

finance ............................0508

History ...............................................

Labor ………................................. 0510

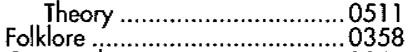

Geography ...................................... 0366

History

General

.0578
History of .............................0320

Archaeology ….....................0324

\section{THE SCIENCES AND ENGINEERINC}

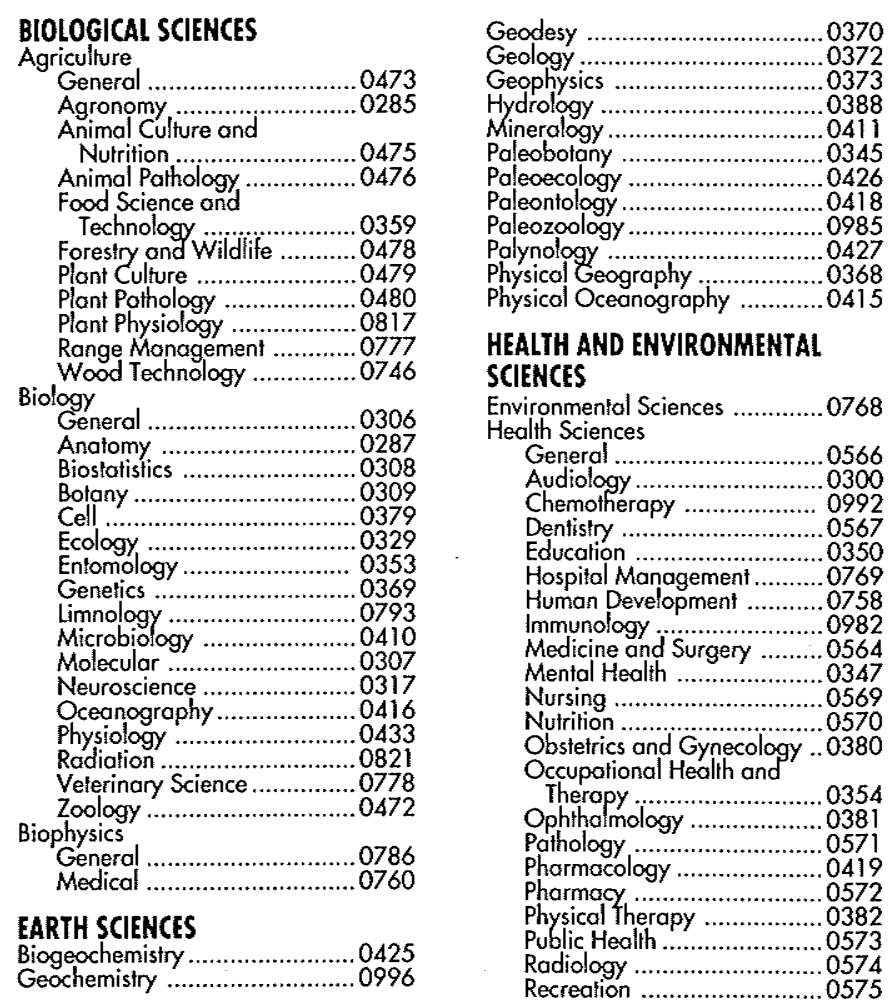

Applied Sciences

Applied Mechanics .....................0346

Computer Science .......................... 0984

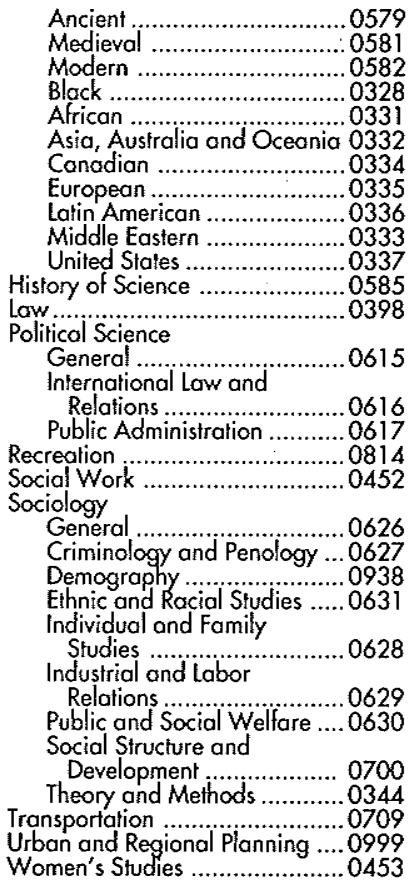

Engineering

General .... 0537

Aerospace ………….....0538

Agriculturol ..............................0539

Automotive ……......................... 0540

Biomedical .............................. 0541

Chemical .............................. 0542

Electronics and Electrical .......0544

Heat and Thermodynamics ... 0348

Hydraulic ............................0545

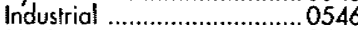

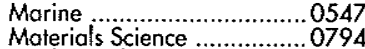

Mechanical ...........................0548

Metallurgy …….....................0743

Mining …….......................0551

Nuclear ............

Packaging ……................. 0549

Petroleum ..........................

System Science .....................0790

Geotechnology ..........................0428

Plastics Technology ……….... 0795

Textile Technology ........................0994

PSYCHOLOGY

General 0621

Behavioral ........................................038

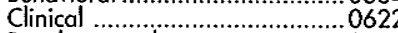

Developmental ................................ 0620

Experimental ...........................0623

Industrial ................................0624

Personality ..................................0625

Physiological .............................0989

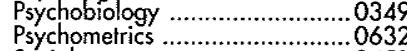

Social .............................................045 


\section{MICROSCOPIC ANALYSIS OF}

HIGH FORCHHEIMER NUMBER FLOW IN POROUS MEDIA

BY

HUIPING MA

A Thesis submitted to the Faculty of Graduate Studies of the University of Manitoba in partial fulfillment of the requirements of the degree of

\section{DOCTOR OF PHILOSOPHY}

(ㄷ) 1994

Permission has been granted to the LIBRARY OF THE UNTVERSITY OF MANTTO BA to lend or sell copies of this thesis, to the NATIONAL LIBRARY OF CANADA to microfilm this thesis and to lend or sell copies of the film, and LIBRARY MICROFILMS to publish an abstract of this thesis.

The author reserves other publication rights, and neither the thesis nor extensive extracts from it may be printed or other-wise reproduced without the author's written. permission. 


\section{ABSTRACT}

In order to achieve a better understanding and accurate predictions of high Forchheimer number flow ('high velocity flow' or 'high Reynolds number flow' as referred to in some literature) in porous media, the research work reported in this dissertation focuses on the quantitative analysis of the pore scale flow mechanisms and the macroscopic effects of microscopic phenomena.

The mathematical model of the problem is formulated using the volumetric averaging method which establishes a relationship between the microscopic and the macroscopic quantities. A new form of the Forchheimer equation and a new dimensionless parameter, the Forchheimer number, are proposed to analyze the nonlinearity in high Forchheimer number flow. Based on physical and mathematical considerations, the precise hydrodynamic definitions of the macroscopic coefficients are derived in terms of the porous microstructure and the pore flow variables. The flow through a periodic diverging-converging capillary is taken as a model problem for gaining further insight into many high Forchheimer number flow problems of practical interest.

In addition to the theoretical approach, this research has also made efforts to quantitatively analyze the relationship between the phenomena at the microscopic and the macroscopic levels. A numerical method and a general-purpose computer program have been developed for modelling pore flow and the transport parameters in porous media. To obtain reliable microscopic flow fields, two numerical schemes have been developed to deal efficiently with the corner vorticity singularity and the pressure computation. The validity of the algorithms is tested by comparing 
the numerical results with physical experiments available in the literature and conducted by this research.

The detailed information for the pore flow, in association with the macroscopic quantities, make it possible to quantitatively demonstrate that the microscopic inertial effect, which leads to distorted velocity and pressure fields, is the fundamental reason for the onset of nonlinearity in high Forchheimer number flow. The systematic computations for different porous configurations and flow conditions have revealed diversified flow patterns and, accordingly, different macroscopic transport behaviours. The correlations of the macroscopic quantities with microscopic level variables have been performed to improve the understanding of the physical mechanism and the constitutive simulation for high Forchheimer number porous media flow. 


\section{ACKNOWLEDGEMENTS}

The author wishes to thank many people who have helped bring this work to completion. First and foremost, he is grateful to his advisor, Dr. Douglas W. Ruth for his continuous guidance and inspiration throughout the course of this research. Thanks are also due to committee members Dr. Ram S. Azad and Dr. Allan D. Woodbury for reading the manuscript and offering valuable suggestions. He is highly appreciative of the external examiner Dr. Jacob H. Masliyah for his comments and suggestions. Mr. Terry Pohjoisrinne rendered helpful assistance in fabricating the experimental facility. The author held a University of Manitoba Post Graduate Fellowship during the research and this support is acknowledged. Last, but not least, thanks to my family, my friends, my wife, Ying, and my son, Jun, for their sustaining affection and support. 


\section{NOMENCLATURE}

\section{ENGLISH}

\begin{tabular}{ll}
$a_{i}$ & body force acceleration $\left(\mathrm{m} / \mathrm{s}^{2}\right)$ \\
$A$ & viscous integral term defined in $(3.34)$ \\
$A_{b}$ & surface bounding the averaging volume $\left(\mathrm{m}^{2}\right)$ \\
$A_{f}$ & area of entrance and exit of a diverging-converging RUC $\left(\mathrm{m}^{2}\right)$ \\
$A_{f f}$ & part of the surface $A_{b}\left(\mathrm{~m}^{2}\right)$ \\
$A_{f}$ & interfacial area between the fluid phase the solid phase $\left(\mathrm{m}^{2}\right)$ \\
$B$ & pressure integral term defined in $(3.32)$ \\
$C_{d}$ & drag coefficient in $(2.8)$ \\
$C_{f}$ & viscous coefficient in $(2.8)$ \\
$d$ & throat diameter of RUC $(\mathrm{m})$ \\
$d_{c}$ & average diameter in $(2.11)$ \\
$D$ & pore diameter of RUC $(\mathrm{m})$ \\
$D_{c}$ & characteristic length scale $(\mathrm{m})$ \\
$\mathbf{e}_{i}$ & microscopic unit vector \\
$F o$ & Forchheimer number defined in $(3.28)$ \\
$\mathbf{g}$ & gravitational acceleration $\left(\mathrm{m} / \mathrm{s}^{2}\right)$ \\
$G_{i}$ & decay function defined in $(5.15)$ \\
$G_{j}$ & decay function defined in $(5.15)$ \\
$i, j$ & finite difference grid indexes \\
$k_{v}$ & microscopic unit vector for RUC \\
$l$ & Darcy's law permeability $\left(\mathrm{m}^{2}\right)$ \\
& characteristic length for the fluid scale $(\mathrm{m})$ \\
\hline &
\end{tabular}




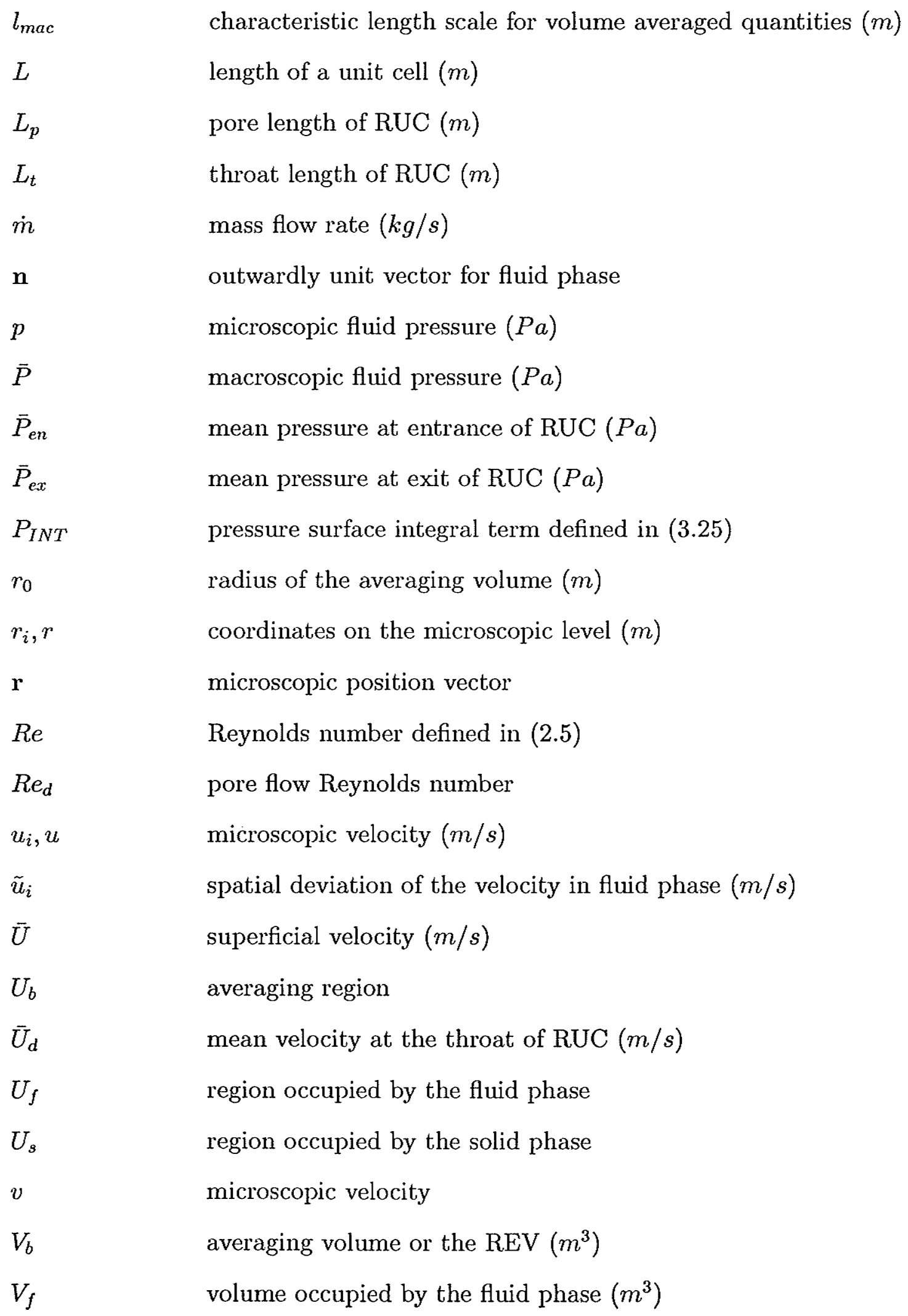




$\begin{array}{ll}V_{s} & \text { volume occupied by the solid phase }\left(\mathrm{m}^{3}\right) \\ V_{I N T} & \text { viscous surface integral term defined in }(3.24) \\ x_{i}, x & \text { coordinate on the microscopic level }(m) \\ X_{i}, X & \text { coordinate on the macroscopic level }(m) \\ \mathbf{X}_{0} & \text { macroscopic vector position of the centroid of the REV } \\ \mathbf{X} & \text { macroscopic position vector }\end{array}$

\section{GREEK}

$\begin{array}{ll}\alpha & \text { void distribution function } \\ \beta & \text { inertial coefficient }(1 / \mathrm{m}) \\ \Gamma^{*} & \text { dimensionless circulation defined in }(5.10) \\ \Delta p_{e}^{*} & \text { excess pressure drop } \\ \Delta \bar{P}^{*} & \text { average pressure loss through an RUC } \\ \epsilon_{p} & \text { error function for pressure computation } \\ \epsilon_{S} & \text { error function for vorticity computation } \\ \kappa & \text { Hagenbach correction } \\ \kappa^{\prime} & \text { Couette correction } \\ \mu & \text { dynamic viscosity of the fluid }\left(N s / \mathrm{m}^{2}\right) \\ \nu & \text { coordinate stretching parameter } \\ \xi & \text { areosity at the entrance and the exit cross section of RUC } \\ \xi_{p} & \text { excess momentum loss factor defined in }(8.1) \\ \rho & \text { fluid density }\left(\mathrm{kg} / \mathrm{m}^{3}\right) \\ \phi & \text { porosity } \\ \psi & \text { stream function } \\ \psi_{f} & \text { a general property of the fluid phase } \\ \Omega & \text { vorticity }\end{array}$


SYMBOLS

$<>\quad$ phase average defined in (3.3)

$<>^{f}$

intrinsic phase average defined in (3.4)

$\tilde{\psi}_{f}$

deviation from intrinsic phase average

$\psi_{f}^{*}$

dimensionless quantities

$\bar{\psi}_{f}$

mean values 


\section{CONTENTS}

ABSTRACT i

ACKNOWLEDGEMENTS

NOMENCLATURE IV

LIST OF FIGURES - xii

LIST OF TABLES Xviii

1. INTRODUCTION $\ldots \ldots \ldots \ldots \ldots \ldots \ldots \ldots \ldots \ldots \ldots \ldots$

1.1 Scaling Up Processes and Problems $\ldots \ldots \ldots \ldots \ldots \ldots \ldots \ldots$

1.2 High Forchheimer Number Flow $\ldots \ldots \ldots \ldots \ldots \ldots \ldots \ldots \ldots \ldots$

1.3 Objectives of the Present Study $\ldots \ldots \ldots \ldots \ldots \ldots \ldots \ldots \ldots$

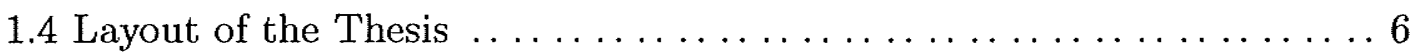

2. REVIEW OF LITERATURE $\ldots \ldots \ldots \ldots \ldots \ldots \ldots \ldots \ldots$

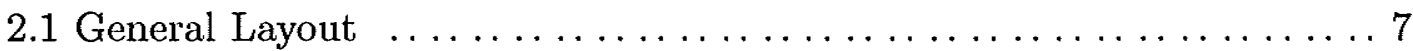

2.2 Phenomenological Models . . . . . . . . . . . . . . . . . . 8

2.3 The Volumetric Averaging Method ................... 14

2.3.1 Deterministic Derivation of the Forchheimer Equation $\ldots \ldots 16$

2.3.2 Mechanism of the Nonlinearity . . . . . . . . . . . . 17

2.3.3 Averaging Theorem and Scale Analysis .............. 19

2.3.4 Closure of the Averaging Procedure $\ldots \ldots \ldots \ldots \ldots \ldots \ldots$

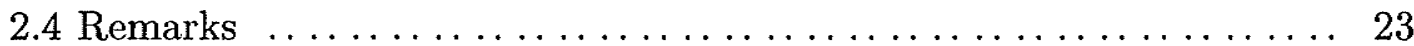

\section{MACROSCOPIC DESCRIPTIONS OF POROUS}

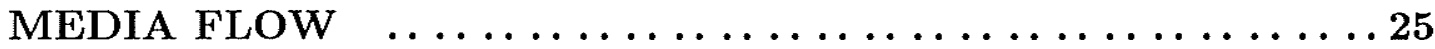

3.1 Introduction to the Volumetric Averaging Method .......... 25

3.2 Macroscopic Momentum Equations $\ldots \ldots \ldots \ldots \ldots \ldots \ldots \ldots \ldots$

3.2.1 Momentum Equation for Heterogeneous Media . . . . . . . . . . 31

3.2.2 Momentum Equation for Homogeneous Media . . . . . . . . . 34 
3.2.3 Momentum Equation for a Periodic Diverging-Converging Capillary Model ........................ 34

3.2.4 Expressions for the Interfacial Drag Force $\ldots \ldots \ldots \ldots \ldots \ldots . . \ldots 9$

3.3 The Forchheimer Number $\ldots \ldots \ldots \ldots \ldots \ldots \ldots \ldots \ldots \ldots \ldots \ldots$

3.4 Hydrodynamic Definitions of Macroscopic Coefficients $\ldots \ldots \ldots \ldots 42$

4. MATHEMATICAL MODEL FOR MICROSCOPIC

FLOW ANALYSIS $\ldots \ldots \ldots \ldots \ldots \ldots \ldots \ldots \ldots \ldots \ldots \ldots$

4.1 Geometry of the Solution Domain $\ldots \ldots \ldots \ldots \ldots \ldots \ldots \ldots \ldots 45$

4.2 Governing Equations for Microscopic Flow $\ldots \ldots \ldots \ldots \ldots \ldots \ldots 4$

4.2.1 Vorticity-Stream Function Approach . . . . . . . . . . . .. 49

4.2.2 Recovery of the Pressure Fields $\ldots \ldots \ldots \ldots \ldots \ldots \ldots \ldots 50$

4.3 Implementation of Boundary Conditions $\ldots \ldots \ldots \ldots \ldots \ldots \ldots . \ldots 2$

4.3.1 Fluid/Solid Interface Boundary Condition ............ 52

4.3.2 Entrance and Exit Condition $\ldots \ldots \ldots \ldots \ldots \ldots \ldots \ldots \ldots$

4.3.3 The Symmetry Condition $\ldots \ldots \ldots \ldots \ldots \ldots \ldots \ldots \ldots . \ldots 5$

4.3.4 Condition at the Sharp Convex Corner $\ldots \ldots \ldots \ldots \ldots \ldots .55$

\section{NUMERICAL SCHEME FOR PORE FLOW}

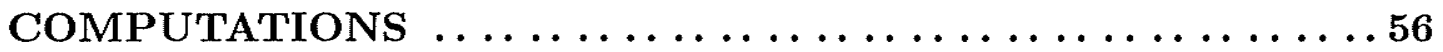

5.1 Treatment of the Corner Singularity $\ldots \ldots \ldots \ldots \ldots \ldots \ldots \ldots . \ldots 6$

5.1 .1 Traditional Corner Methods . ................. 57

5.1.1.1 Ad Hoc Method ........................ 58

5.1.1.2 Moffatt Expansion Method $\ldots \ldots \ldots \ldots \ldots \ldots 61$

5.1.2 The Vorticity-Circulation Method ............... 62

5.1.3 Validity of the Vorticity-Circulation Method ............ 64

5.2 Pressure Recovery: The Hybrid Method $\ldots \ldots \ldots \ldots \ldots \ldots \ldots .65$

5.2.1 The Conventional Methods $\ldots \ldots \ldots \ldots \ldots \ldots \ldots \ldots \ldots 65$

5.2 .2 The Hybrid Method $\ldots \ldots \ldots \ldots \ldots \ldots \ldots \ldots \ldots \ldots$

5.3 Computational Procedure for Flow Equations $\ldots \ldots \ldots \ldots \ldots \ldots 71$ 
5.3.1 The Finite Difference Formulation $\ldots \ldots \ldots \ldots \ldots \ldots$

5.3 .2 Procedures of the Iteration Process . . . . . . . . . . . . 73

5.3 .3 Outline of the Solution Procedure $\ldots \ldots \ldots \ldots \ldots \ldots \ldots 74$

6. COMPUTATIONAL DETAILS $\ldots \ldots \ldots \ldots \ldots \ldots \ldots \ldots$

6.1 Computational Grids $\ldots \ldots \ldots \ldots \ldots \ldots \ldots \ldots \ldots \ldots \ldots \ldots$

6.2 Accuracy of the Finite-Difference Solutions $\ldots \ldots \ldots \ldots \ldots \ldots$

6.2.1 Accuracy of the Vorticity-Circulation Method ... . . . . . . 79

6.2 .2 Accuracy Test for Pressure Computations . . . . . . . . . 81

6.2.3 Accuracy Confirmation of the Numerical Results ......... 83

6.3 Convergence of the Solutions $\ldots \ldots \ldots \ldots \ldots \ldots \ldots \ldots$

6.4 Computer Program $\ldots \ldots \ldots \ldots \ldots \ldots \ldots \ldots \ldots \ldots \ldots \ldots$

7. FLOW MEASUREMENTS $\ldots \ldots \ldots \ldots \ldots \ldots \ldots \ldots \ldots \ldots \ldots \ldots$

7.1 Introduction $\ldots \ldots \ldots \ldots \ldots \ldots \ldots \ldots \ldots \ldots \ldots \ldots \ldots \ldots$

7.2 Experimental Apparatus $\ldots \ldots \ldots \ldots \ldots \ldots \ldots \ldots \ldots \ldots$

7.2.1 Geometry of the Sudden Contraction Test Section . . . . . . 94

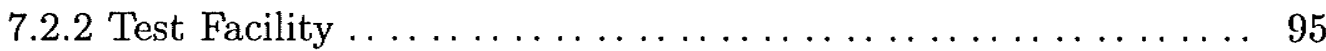

7.3 Experimental Accuracy $\ldots \ldots \ldots \ldots \ldots \ldots \ldots \ldots \ldots \ldots \ldots$

7.4 Result Analysis and Comparison ................... 98

7.4.1 Data Reduction $\ldots \ldots \ldots \ldots \ldots \ldots \ldots \ldots \ldots \ldots \ldots$

7.4.2 Pressure Distributions $\ldots \ldots \ldots \ldots \ldots \ldots \ldots \ldots \ldots \ldots$

7.4.3 Comparisons of the Excess Pressure Drop . . . . . . . . . . . . 100

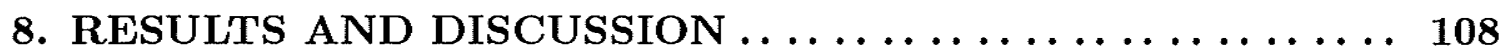

8.1 Microscopic Flow Mechanisms . . . . . . . . . . . . . . . . . 109

8.1.1 Streamlines and Vorticity Contours . . . . . . . . . . . . 109

8.1 .2 Velocity Fields .. . . . . . . . . . . . . . . . . . . . 110

8.1.3 Pressure Distributions $\ldots \ldots \ldots \ldots \ldots \ldots \ldots \ldots \ldots \ldots \ldots$

8.2 Behavior of Macroscopic Properties $\ldots \ldots \ldots \ldots \ldots \ldots \ldots \ldots$

8.2.1 Reynolds Number Effect on $k_{v}$ and $F_{o} \ldots \ldots \ldots \ldots \ldots$ 
8.2.2 Average Pressure Loss $\Delta \bar{P}^{*} \ldots \ldots \ldots \ldots \ldots \ldots \ldots \ldots \ldots \ldots \ldots \ldots$

8.2.3 Interfacial Drag Force........................ 115

8.2.4 Geometric Effects on $k_{v}, F o$ and $\beta \ldots \ldots \ldots \ldots \ldots \ldots \ldots$

8.3 Nonlinear Effect at High F-Number Flow . . . . . . . . . . . . 118

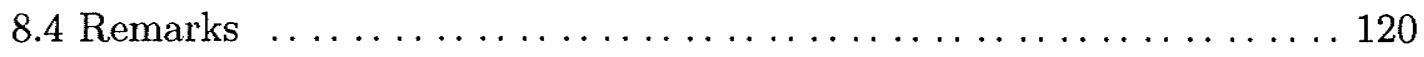

9. CONCLUSIONS AND FUTURE WORK $\ldots \ldots \ldots \ldots \ldots \ldots 177$

9.1 Accomplishments of the Present Research . . . . . . . . . . . . 177

9.2 Recommendations for Future Work $\ldots \ldots \ldots \ldots \ldots \ldots \ldots \ldots 179$ APPENDICES

A. AVERAGING OF THE VISCOUS TERM ......... 181

B. ANALYSIS OF THE DISPERSION TERM $\ldots \ldots \ldots \ldots 183$

C. DERIVATION OF THE PRESSURE TERM ........ 186

D. THE ITERATIVE COEFFICIENTS $\ldots \ldots \ldots \ldots \ldots \ldots 187$

E. UNCERTAINTY IN REYNOLDS NUMBER........ 190

REFERENCES ............................ 191 


\section{LIST OF FIGURES}

FIGURE

PAGE

2.1 Blick's capillary orifice model (Blick, 1966$) \ldots \ldots \ldots \ldots \ldots \ldots \ldots \ldots 12$

2.2 Definition of the different base cells (Coulaud et al., 1988) ......... 13

3.1 A schematic averaging volume comprising a fluid phase and

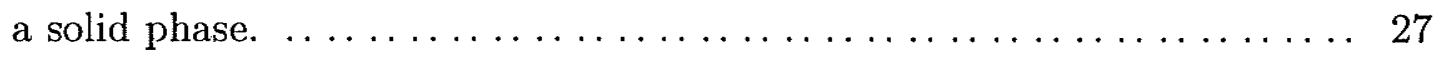

3.2 The periodic diverging-converging capillary model............. 36

3.3 The schematic of a periodic diverging-converging representative unit cell (RUC) $\ldots \ldots \ldots \ldots \ldots \ldots \ldots \ldots \ldots \ldots \ldots, 37$

4.1 A representative unit cell. ........................ 47

4.2 Geometry of the solution domain. $\ldots \ldots \ldots \ldots \ldots \ldots \ldots \ldots \ldots \ldots 48$

5.1 The notation for corner vorticity computations $\ldots \ldots \ldots \ldots \ldots \ldots \ldots 9$

5.2 A schematic of the vorticity-circulation scheme near

a convex corner. . . . . . . . . . . . . . . . . . . . . . . 63

5.3 A comparison of the axial velocity profiles, $R e_{d}=43 \ldots \ldots \ldots \ldots \ldots 66$

$5.4 \mathrm{~A}$ comparison of the axial velocity profiles, $R e_{d}=1813 \ldots \ldots \ldots \ldots 67$

5.5 Comparison of computed and measured profiles of axial velocity for $R e_{d}=698$.

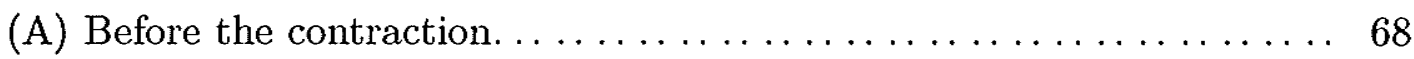

(B) After the contraction. $\ldots \ldots \ldots \ldots \ldots \ldots \ldots \ldots \ldots \ldots \ldots \ldots$

5.6 Flow chart of the numerical solution procedure. $\ldots \ldots \ldots \ldots \ldots \ldots 75$

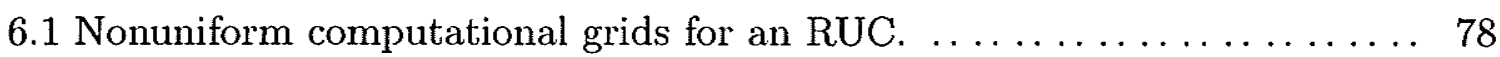

6.2 Variations of the terms in Equation (4.1).

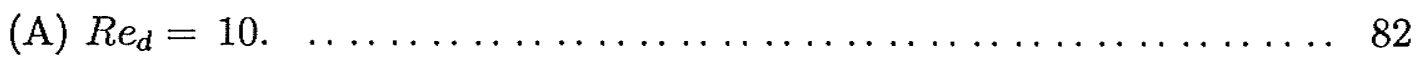

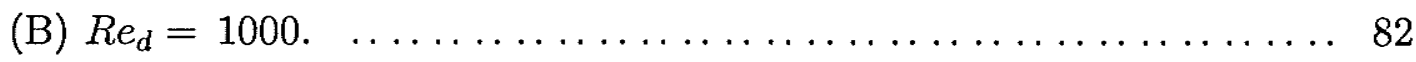

xii 
6.3 Wall vorticity distributions in an RUC for various grids.

(A) The upstream small pipe. $\quad \ldots \ldots \ldots \ldots \ldots \ldots \ldots \ldots \ldots \ldots$

(B) The large pipe. $\ldots \ldots \ldots \ldots \ldots \ldots \ldots \ldots \ldots \ldots \ldots \ldots \ldots \ldots$

(C) The downstream small pipe. $\ldots \ldots \ldots \ldots \ldots \ldots \ldots \ldots \ldots \ldots$

6.4 The converging characteristics of the vorticity-circulation method. . . . . 88

7.1 Apparatus used to measure pressure distributions across the

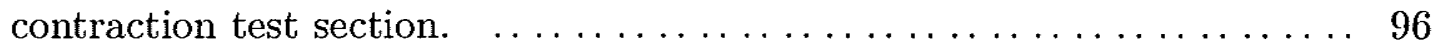

7.2 Representative wall pressure distributions in the small pipe

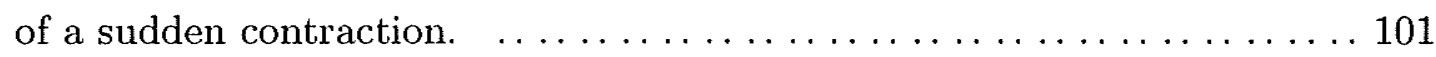

7.3 Comparisons of wall pressure distributions in the small pipe of a sudden contraction flow.

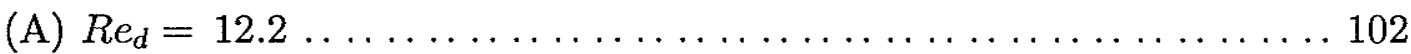

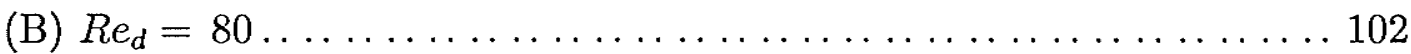

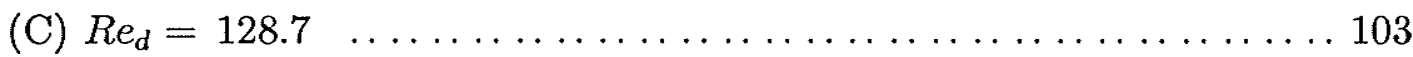

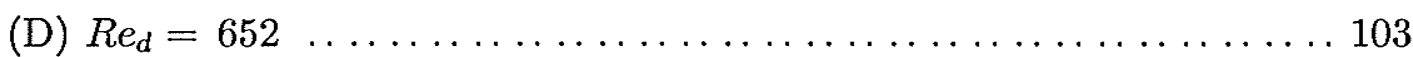

7.4 The excess pressure drop through a sudden contraction, $D / d=8.0$. $\ldots 104$ 7.5 The excess pressure drop through a sudden contraction, $D / d=2.5$. $\ldots 105$

8.1 Geometric configurations of the RUCs.

(A) RUCs with diameter ratio $(D / d)$ changes only. $\ldots \ldots \ldots \ldots \ldots \ldots 122$

(B) RUCs with length ratio $\left(L_{t} / L_{p}\right)$ changes only. $\ldots \ldots \ldots \ldots \ldots \ldots 122$

(C) RUCs with length to diameter ratio $(L / d)$ changes only. . . . . . 123

8.2 Streamlines and vorticity contours for the RUC of $D / d=2, L_{t} / L_{p}=2$ and $L / d=4.875$.

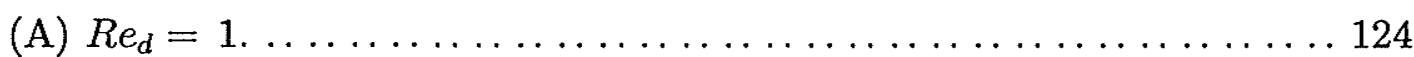

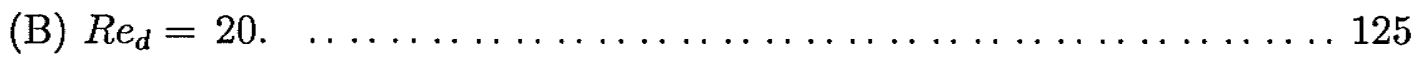

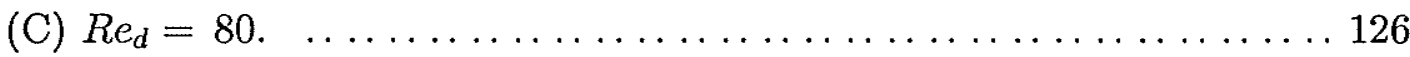

8.3 Flow patterns for the RUC of $D / d=4, L_{t} / L_{p}=2$ and $L / d=4.875$. 


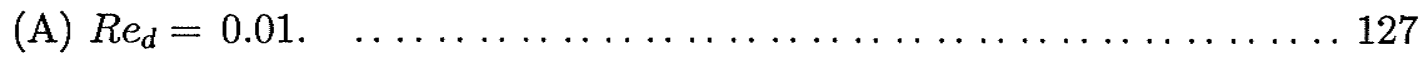

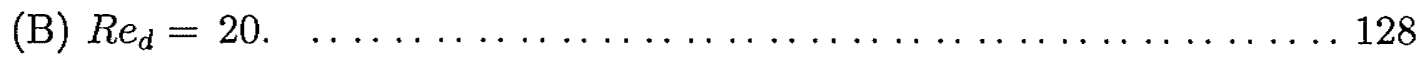

8.4 Flow patterns for the RUC of $D / d=2, L_{t} / L_{p}=4, L / d=4.875$

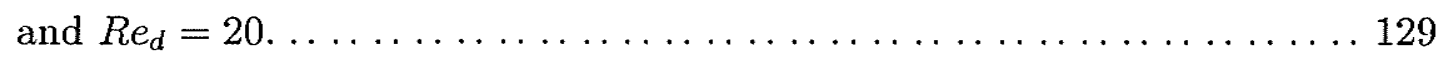

8.5 Distributions of interfacial vorticity for the RUC of $D / d=2$

and $L / d=4.875$.

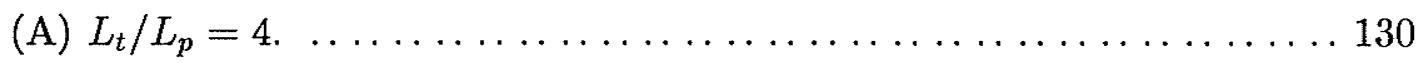

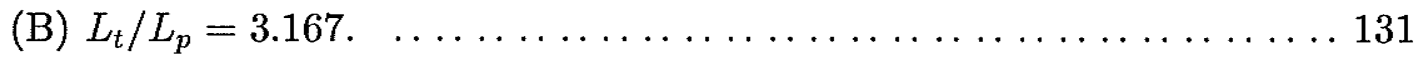

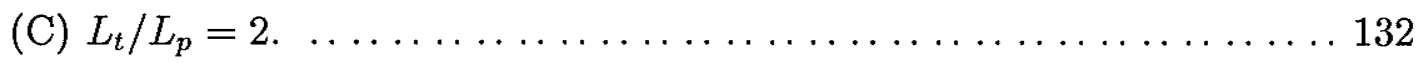

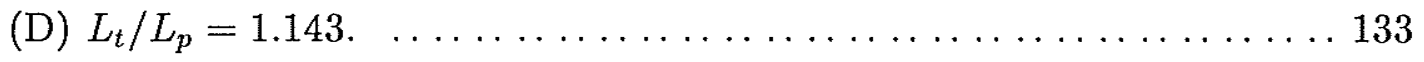

8.6 Centerline velocity distributions for the RUC of $D / d=2$

and $L / d=4.875$.

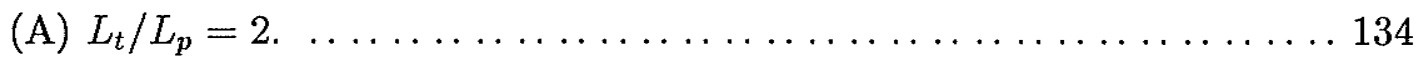

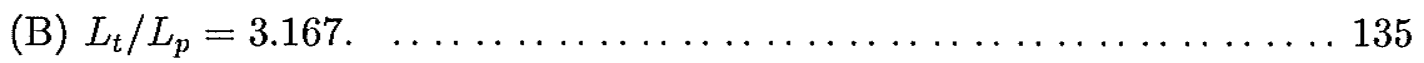

8.7 Velocity profiles at representative axial positions for the RUC of

$D / d=2, L_{t} / L_{p}=2, L / d=4.875$ and $R e_{d}=1$.

(A) Axial velocity profiles. ........................... 136

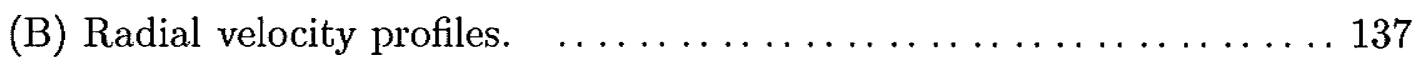

8.8 Velocity profiles at representative axial positions for the RUC of

$D / d=2, L_{t} / L_{p}=2, L / d=4.875$ and $R e_{d}=20$.

(A) Axial velocity profiles. . . . . . . . . . . . . . . . . . 138

(B) Radial velocity profiles. $\ldots \ldots \ldots \ldots \ldots \ldots \ldots \ldots \ldots \ldots \ldots \ldots$

8.9 Velocity profiles at representative axial positions for the RUC of

$D / d=2, L_{t} / L_{p}=2, L / d=4.875$ and $R e_{d}=80$.

(A) Axial velocity profiles. . . . . . . . . . . . . . . . . . 140

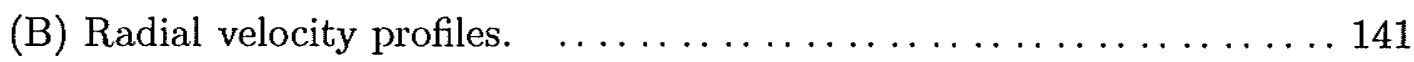

8.10 Velocity field for the RUC of $D / d=1.5, L_{t} / L_{p}=2$ and $L / d=4.875$. 


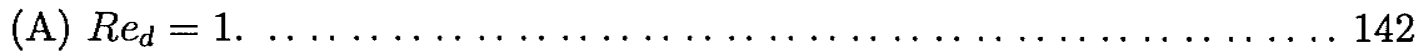

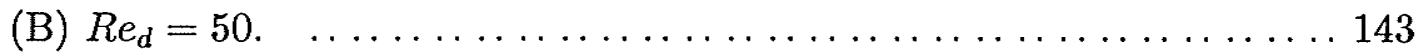

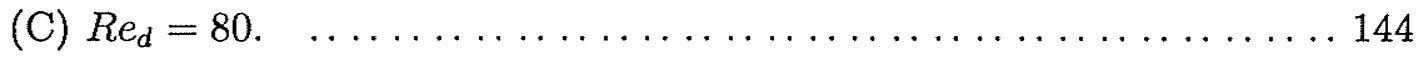

8.11 Velocity field for the RUC of $D / d=3, L_{t} / L_{p}=4$ and $L / d=4.875$.

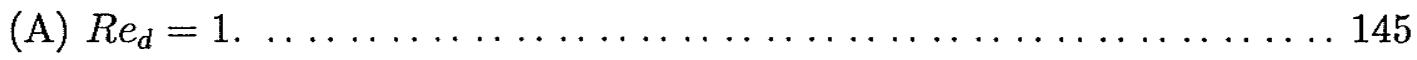

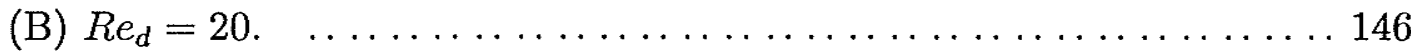

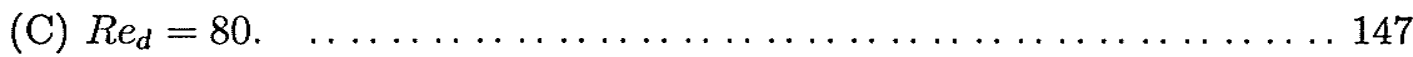

8.12 Velocity field for the RUC of $D / d=4, L_{t} / L_{p}=2$ and $L / d=4.875$.

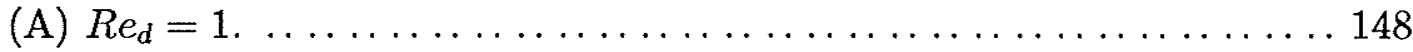

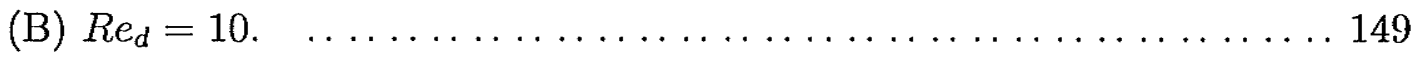

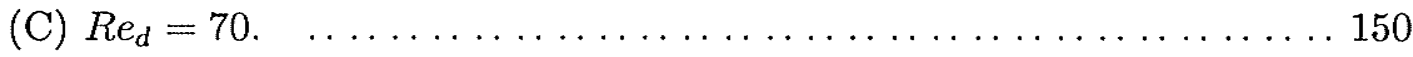

8.13 Pressure distributions at representative axial positions for the

$\mathrm{RUC}$ of $D / d=3, L_{t} / L_{p}=2, L / d=4.875$.

8.14 Mean pressure distributions.
(A) $R e_{d}=1$.
(B) $R e_{d}=10$. 153
(C) $R e_{d}=20$. 154
(D) $R e_{d}=80$. 155

8.15 Effects of length ratio and diameter ratio on $\xi_{p}$ for $R e_{d}=10$ and $L / d=4.875$.

8.16 Variations of Forchheimer number with Reynolds number for the RUC of $L_{t} / L_{p}=2$ and $L / d=4.875$.

8.17 Variations of the velocity dependent permeability with $R e_{d}$ for the RUC of $L_{t} / L_{p}=2$ and $L / d=4.875$. 
8.18 Variations of the average pressure loss for the RUC of $L / d=4.875$.
(A) $R e_{d}=1$.
(B) $R e_{d}=10$. 160
(C) $R e_{d}=20$. 161
(D) $R e_{d}=80$. 162

8.19 Effects of the pore parameters on the viscous integral term for $L / d=4.875$.
(A) $R e_{d}=1$. 163
(B) $R e_{d}=10$. 163
(C) $R e_{d}=20$. 164
(D) $R e_{d}=80$. 164

8.20 Effects of the pore parameters on the pressure integral term for $L / d=4.875$.
(A) $R e_{d}=1$. 165
(B) $R e_{d}=10$. 165
(C) $R e_{d}=20$. 166
(D) $R e_{d}=80$. 166

8.21 Effects of the pore Reynolds number on the interfacial drag force for $L / d=4.875$.
(A) $L_{t} / L_{p}=3.167$. 167
(B) $L_{t} / L_{p}=1.143$. 167

8.22 Effects of the length ratio $\left(L_{t} / L_{p}\right)$ on the interfacial drag force for $L / d=4.875$.
(A) $R e_{d}=1$. 168
(B) $R e_{d}=10$. 168

8.23 Geometric effects on the velocity dependent permeability for $D / d=4, L / d=4.875$ and $A_{b}^{*}=4.5 * 4.5$. 
8.24 Geometric effects on the Forchheimer number for $D / d=3$ and and $L / d=4.875$.

8.25 Geometric effects on the velocity dependent permeability for

$D / d=2, L / d=4.875$ and $A_{b}^{*}=4.5 * 4.5$.

8.26 Effects of the length to diameter ratio on the velocity dependent

permeability for $D / d=2$ and $L_{t} / L_{p}=2$.

8.27 Porosity effects on the velocity dependent permeability for $D / d=2$,

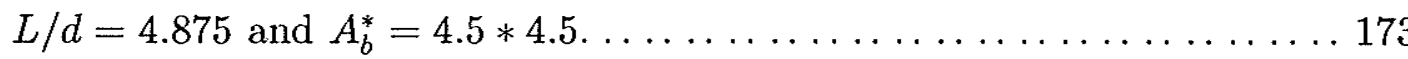

8.28 Balance of the forces along the capillary for the RUC of $D / d=2$,

$L_{t} / L_{p}=2$ and $L / d=4.875$.

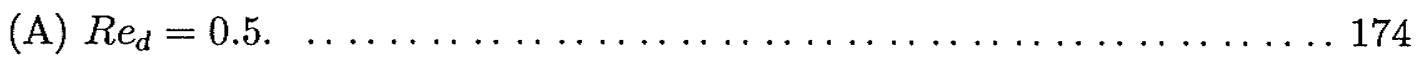

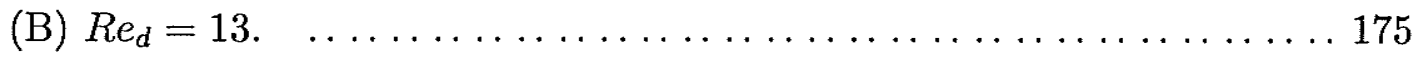

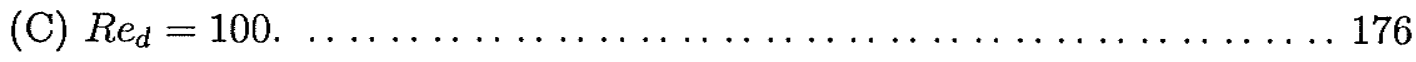

xvii 


\section{LIST OF TABLES}

TABLE

PAGE

6.1 Conservation test of the vorticity-circulation method for $R e_{d}=43 . \quad \ldots \quad 80$

6.2 Conservation test of the vorticity-circulation method for $R e_{d}=1813 . \quad$.. 80

7.1 Dimensions of the test sections. $\ldots \ldots \ldots \ldots \ldots \ldots \ldots \ldots \ldots \ldots \ldots$

7.2 Axial locations of the pressure taps. $\ldots \ldots \ldots \ldots \ldots \ldots \ldots \ldots \ldots 94$

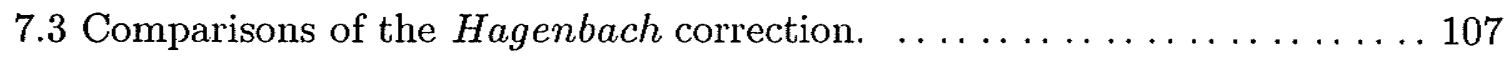

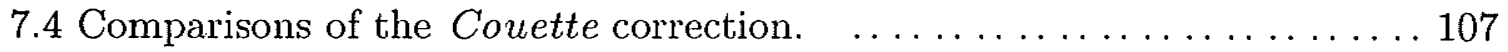

8.1 The geometric effects on the inertial coefficient $\beta$ for

the RUC of $L / d=4.875 \ldots \ldots \ldots \ldots \ldots \ldots \ldots \ldots \ldots \ldots \ldots \ldots \ldots$ 


\section{CHAPTER 1}

\section{INTRODUCTION}

The understanding of the nature of fluid flow in porous media is essential for many science and engineering applications. Such diversified fields as petroleum recovery, ground water hydrology, water purification, powder metallurgy, filtration and drying to name just a few, need to understand transport phenomena in porous media. One of the challenges associated with analyzing transport phenomena is the study of 'high velocity flow' (high Forchheimer number flow as defined more precisely in this study).

The present study, which mainly emphasizes numerical analysis of the transport phenomena at both microscopic and macroscopic levels and of the quantitative relations of the variables at these two levels, is aimed at improving the understanding of the physics of high Forchheimer flow in porous media, based on the volumetric averaging method. To facilitate a focused study, a porous medium is defined in this study as a multiphase material body:

- throughout which both a persistent solid matrix and a void space are present;

- within which the void space is at least partly interconnected and contains one or more fluid phases;

- over which the fluids are able to penetrate.

Flow of fluids through the porous medium defined above will give rise to exchanges of mass, momentum and energy between different phases and between the porous 
medium and the environment. A good knowledge of the physical nature of these transport phenomena and a correct, quantitative description of these processes will be beneficial to engineering and science applications which are subject to the same fundamental laws governing flow through porous media.

\subsection{Scaling Up Processes and Problems}

In principle, the above mentioned transport phenomena in a porous medium of practical interest might be treated at the level where the fluid phase(s) in the pores of the medium is (are) considered to be continuous. However, at this level (usually called the microscopic level or pore scale), the accurate description of the complicated geometry of the interphase boundaries is an insurmountable barrier for the mathematical tools and the computational resources presently available. To circumvent this difficulty, some scaling up methods have been developed in the last two decades (see Chapter 2 for details). According to these methods, the transport phenomena are described, in one way or another, at a level (usually called the macroscopic level or laboratory scale) with a characteristic length scale which is much larger than the corresponding pore scale. The quantities defined at the macroscopic level are thought to be differentiable at each mathematical point and to be more consistent with today's ability to observe the fluid movement.

As an expense of passing from the microscopic level to the macroscopic level, the particular details of the intricate variations of the fluid state variables associated with the microstructure of a medium are smoothed out, and as will be seen in the following chapters, the main characteristic effects of the interaction between different phases at the pore scale are retained in the form of coefficients at the higher level in various degrees depending on the scaling up processes. A full un- 
derstanding of the characteristics of these coefficient, such as their structures and relationships to the statistical properties of the pore configuration of the medium, is essential in the analysis of porous media flow. It is also obvious that to achieve any solutions of a problem at the macroscopic level, these coefficients, which represent the particularity of a specific system, must be first effectively modelled and quantitatively determined for the given problem. As a matter of fact, because of the strong dependence of the macroscopic phenomena on microscopic flow mechanisms and the extreme complexity of the fluid flow within the pore system, the analysis and determination of these coefficients constitutes one of the most challenging problems of transport in porous media.

In this study, the volumetric averaging method (continuum approach), one of the most often used scaling up processes, has been utilized for the reasons presented in Chapter 3.

\subsection{High Forchheimer Number Flow}

On the macroscopic level, fluid flow in a porous medium at low superficial velocity (specific discharge) is generally described by Darcy's law which presents a linear relationship between the driving force and the filtration velocity. However, as the filtration velocity is raised beyond a certain value, numerous experimental observations have confirmed that Darcy's law should be replaced by another timehonored empirical formula, the Forchheimer equation, to account for the nonlinear effects for the medium considered.

In the present research, the terminology, 'high Forchheimer number flow' (abbreviated to high $\mathrm{F}$-number flow in the following), is defined as the situations 
whenever the nonlinear effects become nonnegligible. In the literature, the terms 'high velocity flow' or 'high Reynolds number flow' have also been used to refer to the same circumstances. However, it will be demonstrated that 'high $\mathrm{F}$-number flow' is perhaps the most appropriate term to indicate the essence of the phenomenon.

To be sure, even for the linear case, a strict theory which predicts not only the form of Darcy's law but the macroscopic quantities (the coefficients) is a formidable problem if the real complexity of the pore geometry is considered. The introduction of the nonlinearity in high F-number flow implies that the full Navier-Stokes equations, instead of the Stokes equation in the linear flow, should be initiated and the inertial effects at both microscopic and macroscopic levels should be taken into account.

In the analysis of the high $\mathrm{F}-$ number flow in porous media, whether the physical explanations of the flow phenomena or the quantitative descriptions of the macroscopic properties, a clear picture of the microscopic flow mechanisms is fundamentally important. To acquire this invaluable information, an efficient numerical simulation method is developed in the present research to quantitatively analyze the microscopic flow in the high Forchheimer number regime and the macroscopic effects of the microscopic phenomena when the inertial nonlinearity becomes significant.

\subsection{Objectives of the Present Study}

By means of numerical and experimental analyses, this research aims at improving the understanding of the physical mechanisms and the quantitative descriptions for 
high $\mathrm{F}$-number flow in porous media. To this end, the following concrete objectives were set for the work:

1. To introduce a new form of empirical equation and a new dimensionless parameter, the Forchheimer number, in order to effectively analyze the nonlinear phenomena in high $\mathrm{F}$-number flow;

2. To derive the precise hydrodynamic definitions for the macroscopic coefficients in the Forchheimer equation, in terms of the pore scale quantities by applying the volumetric averaging method;

3. To develop a numerical simulation method along with the computer program which provides reliable numerical solutions for the pore scale variables associated with different microstructure;

4. To perform systematic numerical calculations for a spatially periodic porous media model in order to clarify the physical causes underlying the deviation from linearity of Darcy's law in high F-number flow;

5. To determine both the fields of pore scale quantities and the macroscopic properties in a systematic way for a spatially periodic porous media model in order to correlate the macroscopic quantities with the pore scale variables and microstructure;

6. To measure the flow rate-pressure drop relations experimentally for two cases of sudden contraction flow, with the aim of supporting the reliability of the numerical scheme and clarifying the long standing confusion on the pressure loss of such flow. 


\subsection{Layout of the Thesis}

The thesis is composed of nine chapters. A literature review of studies in high F-number flow is presented in Chapter 2. The general mathematical formulations at both macroscopic and microscopic levels for the present study are derived and analyzed in Chapters 3 and 4 , respectively. Chapters 5 and 6 present the details of the numerical schemes developed for the flow computations. Being an important part of this research, the experimental study of a sudden contraction flow is given in Chapter 7 where comparisons between measurement and prediction are made wherever possible. A full presentation of the numerical results and a discussion of them are the contents of Chapter 8. Finally, conclusions and recommendations for future work are given in Chapter 9. 


\section{CHAPTER 2}

\section{REVIEW OF LITERATURE}

\subsection{General Layout}

The literature review presented in this chapter is limited to the works on high $\mathrm{F}$-number flow in porous media and is intended to provide a clear picture of the background for the present research. For the sake of convenience in presentation, the review is divided into two sections which are reviews of: the phenomenological model (Section 2.2) and the volumetric averaging method (Section 2.3). Areas where further work is most desirable are identified in detail. Although some progress has also been made recently in other procedures in this field, such as the homogenization method (e.g., Sanchez-Palencia, 1980; Ene and Polisevski, 1987; Mei and Auriault, 1991) and the statistical method (e.g., Dagan, 1986, 1990; Gelhar and Axness, 1983), the review has not given much attention to these methods because they are less relevant to the present research. However, for the purpose of comparisons, some typical results of these methods have been discussed briefly in the relevant chapters. Reviews of several special topics, such as the numerical treatments for microscopic flow computations, are not included in this chapter. Instead, they have been given in the chapters where the specific problems are addressed. 


\subsection{Phenomenological Models}

Various empirical and semi-empirical methods have been in use since the end of the nineteenth century to uncover the correlations of porous media transport coefficients with the macroscopic geometric properties of the pore system. The correlations obtained as a result of such procedures are called 'phenomenological models' in this review.

Without concern about the microscopic flow mechanisms, Darcy (1856) pos-

tulated a linear relationship between the macroscopic pressure gradient, $\frac{d \bar{P}}{d X}$, and the filtration velocity, $\bar{U}$, for single phase, one-dimensional flow through a porous medium:

$$
-\frac{d \bar{P}}{d X}=\frac{\mu}{k} \bar{U}
$$

where $\mu$ denotes the dynamic viscosity of the fluid, and $k$ is Darcy's law permeability which represents a measure of the flow conductance of the solid matrix. As the experimental filtration velocity increases, deviations from Darcy's law (2.1) are observed. Various relationships have been proposed to describe the bulk hydrodynamic behavior of the fluid motion in a porous medium at higher filtration velocity (see, Scheidegger, 1960; Bear, 1972; Hannoura and Barends, 1981). The first of such relationships to account for the nonlinear effects was given by Forchheimer (1901) who suggested the following one-dimensional form:

$$
-\frac{d \bar{P}}{d X}=\frac{\mu}{k} \bar{U}+\beta \rho \bar{U}^{2}
$$

where $\rho$ represents the fluid density, and $\beta$ is an experimentally derived parameter called the inertial coefficient. Both $k$ and $\beta$ in Equation (2.2) are the material constants. Although other modified forms have been derived to make the equation fit experimental data better, being a phenomenological description of porous media 
flow, the Forchheimer equation generally agrees sufficiently well with experimental observations in certain velocity ranges. Nevertheless, because it is an empirical formula, the Forchheimer equation does not provide any more information about correlations of the permeability and the inertial coefficient with the geometric properties of porous media than what could be obtained from dimensional considerations. To have a better understanding of the nature of the phenomena and to predict the relations of various properties of the porous media, efforts have been directed toward finding such correlations based on empirical and theoretical considerations (Scheidegger, 1960; Bear, 1972; Dullien, 1979).

Among others, the two most widely accepted correlations are perhaps the Ergun equations for packed beds (Ergun, 1952) which were later modified by Macdonald et al. (1979) through examining much more data than was ever used before by others. The following expressions were found to give the best fit to most of the experimental data:

$$
-\frac{d \bar{P} / d X}{\rho \bar{U}^{2}} D_{c} \frac{\phi^{3}}{1-\phi}=\frac{180(1-\phi)}{R e}+1.8, \quad \text { (For smooth surfaces) }
$$

and

$$
\left.-\frac{d \bar{P} / d X}{\rho \bar{U}^{2}} D_{c} \frac{\phi^{3}}{1-\phi}=\frac{180(1-\phi)}{R e}+4.0, \quad \text { (For rough surfaces }\right)
$$

where $\phi$ is the porosity of the medium and $R e$, the medium Reynolds number, is given by

$$
R e=\frac{\rho \bar{U} D_{c}}{\mu}
$$

and $D_{c}$ is a characteristic length scale of the medium. By comparing Equation (2.3) with the Forchheimer equation (Equation (2.2)), expressions of the Darcy's law permeability and the inertial coefficient for smoothed surfaces, for example, 
can be deduced:

$$
\begin{aligned}
& k=\frac{\phi^{3} D_{c}^{2}}{180(1-\phi)^{2}}, \\
& \beta=\frac{1.8}{D_{c}} \frac{(1-\phi)}{\phi^{3}} .
\end{aligned}
$$

Both $k$ and $\beta$ seem to have been correlated with measurable quantities, but the characteristic length $D_{c}$ is not really defined for a porous medium and its determination cannot be achieved uniquely. Therefore, these semi-empirical equations are subject to the same limitations as those experienced by the hydraulic radius models (Carman-Kozeny theory) which assumed a length termed "hydraulic radius" to be characteristic for the permeability of a porous medium and linked such a length with the hypothetical channels which the porous medium was thought to be equivalent (Kozeny, 1927; Carman, 1937, 1938, 1956).

In addition to the proposed empirical and semi-empirical relations, attempts have been made to deduce the Forchheimer equation from theoretical considerations. For example, Irmay (1958) started from the Navier-Stokes equations, including all the inertial terms, and performed a space average of the equations over a homogeneous, isotropic model of spheres of equal diameters. By assuming that a correlation between microscopic velocity components exists at high filtration velocity, a formula containing the square of velocity was obtained for a macroscopically one-dimensional flow, where the macroscopic coefficients were derived and expressed in terms of geometric parameters of the pore structures. Within the framework of the Carman-Kozeny hydraulic radius theory, the equation given by Irmay (1958) reduced to the exact Carman-Kozeny form at low values of Reynolds number. 
In comparison, some other theoretical derivations of nonlinear flow are based on the microscopic analysis of the flow in an idealized geometrical description of a porous medium. An example of this type of model is that of Blick (1966) which represents a porous medium as a bundle of parallel capillary tubes with orifice plates spaced throughout the tubes at distances equal to the tube diameter (Figure 2.1). A static balance of forces over the control volume is applied to obtain an equation of the Forchheimer type for the case of one-dimensional Newtonian fluid in a rigid medium. The final form of the equation can be written as

$$
-\frac{d \vec{P}}{d X}=2 \frac{C_{f} R e}{\phi \delta^{2}} \mu \vec{U}+0.5 \frac{C_{d}}{\phi^{2} \delta} \rho \bar{U}^{2}
$$

and it follows that Darcy's law permeability and the inertial coefficient are

$$
\begin{gathered}
k=0.5 \frac{\phi \delta^{2}}{C_{f} R e} \\
\beta=0.5 \frac{C_{d}}{\phi^{2} \delta}
\end{gathered}
$$

where $C_{f}$ and $C_{d}$ are the viscous coefficient and the drag coefficient, and $\delta$ is the average pore diameter. To calculate $k$ and $\beta$ by Equations (2.9) and (2.10), the values of $C_{f}$ and $C_{d}$ must be known first, which implies that further assumptions about the interaction between the solid and the fluid phases have to be made. In another investigation, Coulaud et al. (1988) analyzed the nonlinear effects through the use of an empirically introduced term. Their numerical flow simulations at the pore scale were achieved for a porous medium modelled in terms of cylinders arranged in a regular pattern as illustrated in Figure 2.2. Through a data fitting process for the computed results of global pressure drop and average velocity, they arrived at the relationship: 

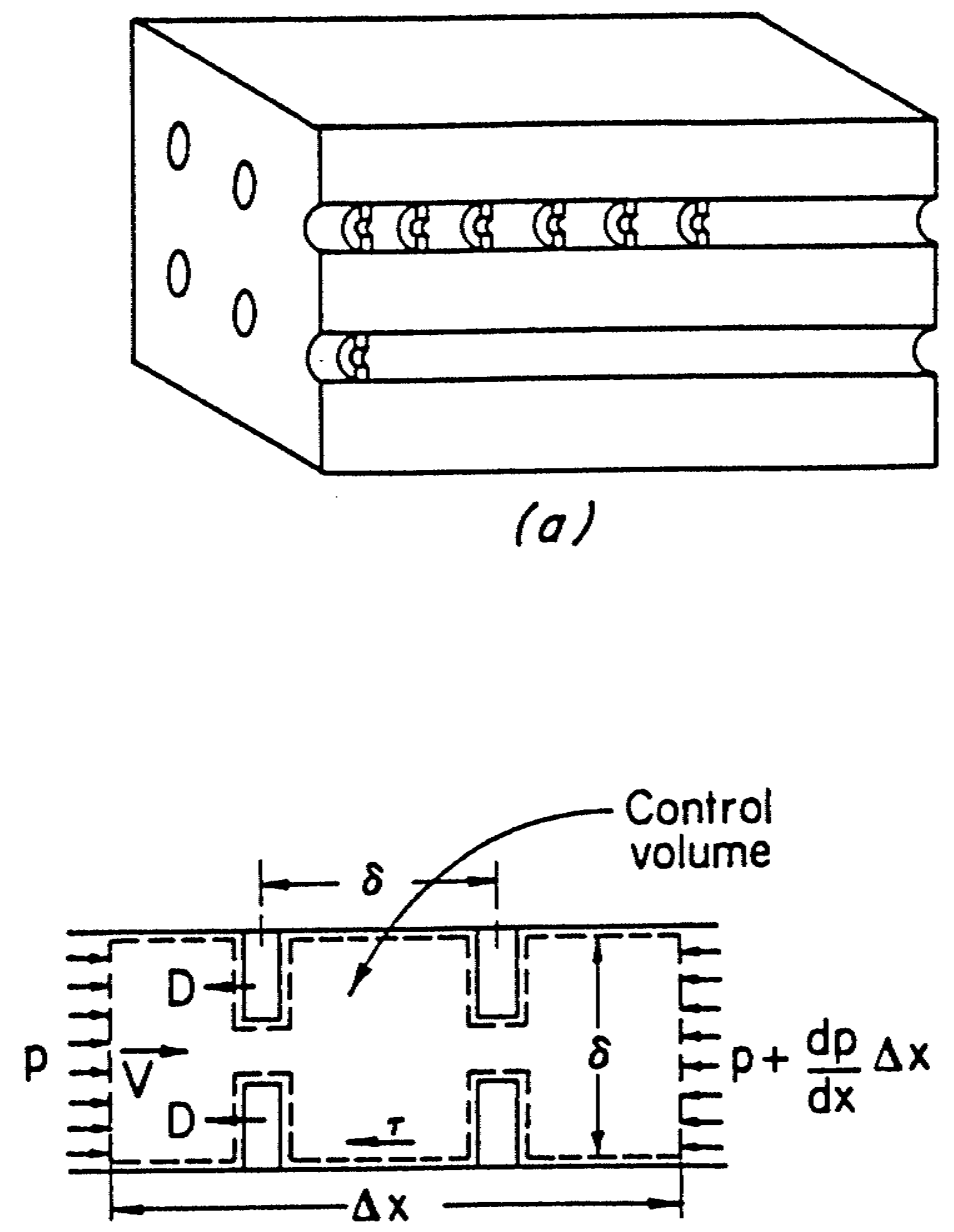

(b)

Fig. 2.1. Blick's capillary orifice model (Blick, 1966). 

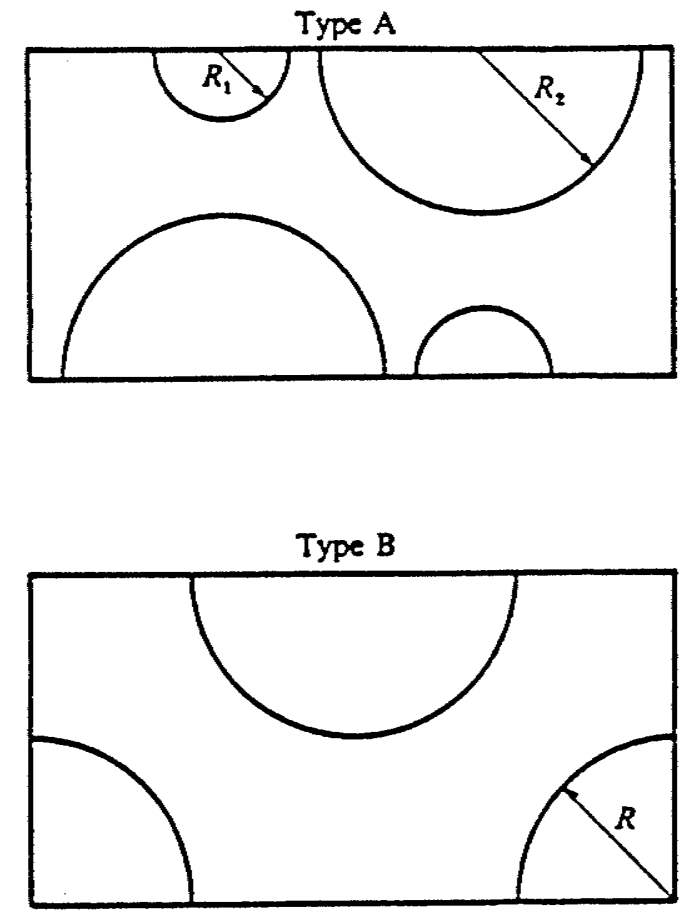

Type C

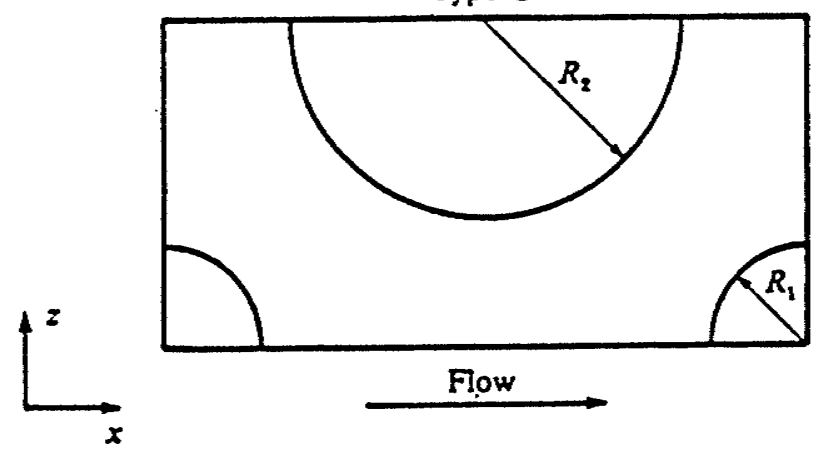

Fig. 2.2. Definition of different base cells (Coulaud et al., 1988). 


$$
-\frac{d \bar{P}}{d X}=a \frac{\alpha}{d_{c}^{2}} \mu \bar{U}+b \frac{\alpha}{d_{c}} \rho \bar{U}^{2}
$$

where $d_{c}$ is the average diameter of the cylinders and $a, b$ and $\alpha$ are parameters depending on the structure of the assumed pore space only.

From the discussions of the phenomenological models so far, it may be deduced that although the correlations reviewed above are useful, in one way or another,

for certain engineering applications of a particular system, they hardly supply a sound theoretical basis for the purpose of universal analysis of the physics of porous media flow since the introduction of various heuristic assumptions. As will be seen in the next section, a more rigorous approach, the volumetric averaging method, has been developed in recent years to provide a more rational and systematic framework within which the mechanics of flow through porous media could be advanced.

\subsection{The Volumetric Averaging Method}

In the past two decades, much attention has been devoted to the volumetric averaging method (the continuum approach) starting from the conservation laws of continuum mechanics applied at the microscopic level. According to this approach, the real porous system, consisting of two (or more) scattered phases, is described by a series of continuous, differentiable variables which are obtained by tracing a Representative Elementary Volume (REV) in the domain of interest. The basic advantages of this continuum approach are (see, e.g., Bear and Bachmat, 1991):

a. The process occurring in porous media is described in terms of differentiable quantities, thus enabling the solution of the problems by employing methods 
of mathematical analysis;

b. The scale of averaged quantities are close to the scale on which experimental measurements are made;

c. The exact configuration of the interphase boundaries need not be specified on the macroscopic level.

However, as will be described in the following analysis, the averaging procedure gives rise to higher order schemes similar to the ones encountered in turbulent flow and closure problems exist for the quantities which represent the effects of the microscopic configuration of interphase boundaries and the actual variations of the state variables within each phase. Recently, a systematic methodology for constructing mathematical models of transport problems in porous domains on the basis of the continuum approach was presented by Bear and Bachmat (1991).

Since the key mathematical theorem which related the average of the gradient and the gradient of the average was proposed independently by Slattery (1967) and Whitaker (1967), there have been many attempts to analyze average behaviour of high F-number flow in porous media (Whitaker, 1969; Carbonell and Whitaker, 1984; Gray and O’Neill, 1976; Hassanazdh and Gray, 1979a,b, 1980, 1987; Cvetković, 1986; Du Plessis and Masliyah, 1988, 1991). Basically, the previous research was concentrated on the topics of

a. the deterministic derivation of the macroscopic governing equations;

b. the closure schemes for the macroscopic thermodynamic quantities;

c. the physical explanations of the mechanisms of the nonlinearity; 
d. the theoretical expressions for the macroscopic coefficients, such as Darcy's law permeability $k$ and the inertial coefficient $\beta$, in the empirical equations.

In the following subsections, the methods of problem solving, rather than details of the results, will be discussed for these topics.

\subsubsection{Deterministic Derivation of the Forchheimer Equation}

As already mentioned, the Forchheimer equation (2.2) is found to be in sufficiently good agreement with experimental evidence for nonlinear flow in porous media (Scheidegger, 1960; Bear, 1972). In order to gain a deep insight into the relationships between the macroscopic phenomena with the microstructure and the microflow variables, many studies have been devoted to the theoretical determination of the Forchheimer equation through the continuum approach. An example of such a derivation is that of Barak and Bear (1981) which considered five different physical models with various degrees of complexity. By using these models, they obtained an approximate expression for the relationship between the pressure gradient and the filtration velocity and compared this expression with their mathematical models and experimental results for the case of saturated, steady and uniform flow of a Newtonian fluid.

In another theoretical derivation, Cvetković (1986) averaged the general form of the linear momentum equation to produce a macroscopic balance equation of motion for fluid flow in porous media. Instead of employing the conventional velocity deviation vector, he introduced a description for the microscopic kinematic field by treating the deviations of local velocity magnitude and direction separately 
and arrived at a generalized momentum equation with a term of second order in the flow velocity. Another interesting example is the work of Dullien and Azzam (1973) in which they applied the averaging theorem to the microscopic momentum equation and obtained a general volume-averaged flow equation. This equation was then cast in a form comparable to the Forchheimer equation. By a direct comparison, they concluded that Darcy's law permeability depends only on the average of the microscopic viscous terms.

Apart from the effort to derive the Forchheimer equation formally, some analyses have been carried one step further by introducing an idealized geometrical description of a porous medium such that the microscopic flow phenomena can be modelled. For example, by using an explicit representative geometric models of the porous medium, Du Plessis and Masliyah (1988) established a direct link between the porosity and tortuosity for their sponge-like material. In this manner, the microstructure of the model could be fully described by two physical parameters, the porosity and a characteristic length. Furthermore, they replaced the interfacial integral term in the macroscopic momentum equation by an empirical formula of the developing flow in a square duct and finally obtained a momentum equation which took the form of the Forchheimer equation as a limiting case. Despite the empirical feature of this work in the treatment of the interfacial integral terms, a good agreement of the values of permeability between the experimental results and the theoretical predictions was reported by Stone and Sawatzky (1990). Following a similar approach, Du Plessis and Masliyah (1991) presented an analysis for laminar flow through a rigid isotropic granular porous medium. 


\subsubsection{Mechanisms of the Nonlinearity}

It has long been a common interest of many researchers to clarify the physical reason for the onset of the nonlinearity in high F-number flow. Early descriptions attributed the nonlinearity to the occurrence of turbulence. However, experiments have indicated that when the macroscopic velocity gradually increases, the nonlinear phenomena appear much before the onset of real turbulence in porous media flow (e.g., Scheidegger, 1960; Bear, 1972; Dybbs and Edwards, 1982). Thus, it can be concluded firmly that the deviations from Darcy's law are not initiated by changes of flow regime.

A diversity of opinions as to why nonlinearity at high flow rates occurs still exists. In their paper, Hassanizadeh and Gray (1987) performed an order of magnitude analysis for the averaged momentum equation and concluded that the microscopic viscous force is the source for the onset of nonlinearity. In contradiction, Barak (1987) ascribed the nonlinearity to microscopic inertial forces through considering the formation of local vortices and development of tortuous streamlines inside pores with increasing pore Reynolds numbers. His point of view was widely accepted among the researchers of this area (e.g., Cvetković, 1986; Du Plessis and Masliyah, 1988, 1991; Coulaud et al., 1988; Mei and Auriault, 1991).

Moreover, explanations of how the microscopic inertial forces manifest themselves on the macroscopic level also differ. Cvetković (1986) attributed the nonlinearity to the dispersion flux (see Chapter 3) and claimed that this term contains most of the information on convective microscopic inertial effects. However, in the investigation by Du Plessis and Masliyah (1988), the macroscopic nonlinearity was obtained even when the dispersion term was ignored.

Although the opinions on the mechanism responsible for the onset of nonlin- 
earity are diverse, the literature has made one point clear-the clarification of the nonlinear physics can be attained only through sophisticated quantitative analysis of the microscopic flow. In the present research, detailed information of the microscopic flow fields, in association with the macroscopic quantities from the averaged momentum balance, have been provided together, with the aim of improving the understanding of the mechanisms of the nonlinearity.

\subsubsection{Averaging Theorem and Scale Analysis}

The spatial averaging theorem (Equation (3.8) in Chapter 3) which relates the local average of a derivative to the derivative of the local average for a function defined in both the solid and the fluid phase and suffering a jump discontinuity at the phase interface has formed the foundation for much of the recent work. To answer the question raised by Veverka (1981), that is whether the volume average is differentiable, Howes and Whitaker (1985) presented a direct derivation of the spatial averaging theorem using a linear transformation and Abel's formula. The examples given in their paper indicated that a spherical averaging volume yielded continuous average functions that are continuously differentiable to any order for systems of practical importance. In a more general manner, Mls (1987) mathematically proved that the first derivatives of the volume average expressed by Equation (3.3) exist almost everywhere in the three-dimensional Euclidien vector space.

Although there seem to be no severe restrictions on the realistic applications of the averaging theorem, the requirement that the difference between the average at any given point within the REV and the average at the centroid can be expressed in a linear relation has imposed certain constraints on the size of the av- 
eraging volume (Whitaker, 1969, 1986; Bachmat and Bear, 1986). Using an order of magnitude analysis, Carbonell and Whitaker (1984) have shown that the radius of the averaging volume, $r_{0}$, and the macroscopic length scale of the medium, $l_{m a c}$, should satisfy the following length scale constraint:

$$
\left(\frac{r_{0}}{l_{m a c}}\right)^{2}<<1 .
$$

It is important to note that the above inequality results from an order of magnitude treatment, and the precise nature of the averaging theorem and the constraint (2.12) still require necessary comparisons between theory and experiment.

To simplify the problem, an order of magnitude comparison has frequently been utilized in the analysis of transport phenomena in porous media (e.g., Whitaker, 1986; Hassanazdh and Gray, 1987). The assumption that the average quantities change significantly in a macroscopic scale ' $l_{\text {mac }}$ ' which is much larger than the microscopic scale ' $l$ ' will always produce the results that some average quantities can be ignored automatically. The only quantitative explanation about the relationship of different scales was given by Whitaker (1986) in his theoretical derivation of Darcy's law. By taking the porosity as a function of $r_{0}$ as a guide, he suggested that to obtain a well behaved porosity function, a requirement of $r_{0} \geq 5 l$ must be satisfied, and on this basis, the averaged quantities undergo significant variations over distances that are at least fifty times larger than $l$. For an accurate description of the physical process, the conditions of validity of the conventional scale assumptions and the relationships between $l_{m a c}, l$ and $r_{0}$ remain to be justified quantitatively.

\subsubsection{Closure of the Averaging Procedure}

After applying the volumetric average for the microscopic governing equations by 
the continuum approach, several terms which contain the important information of the characteristics of the microscopic quantities for a given medium appear in the resulting macroscopic governing equations. To process any solutions of transport problems at the macroscopic level, the constitutive representations for these terms have to be determined first in terms of the averaged variables.

For the linear momentum balance equation at the macroscopic level, the essential point in the continuum approach is the introduction of a velocity deviation $\tilde{u}_{i}$ which represents the difference of pore velocity and its intrinsic phase average (see Chapter 3 for details). Similar to the analysis of turbulent flow, a term $\rho<\tilde{u}_{i} \tilde{u}_{j}>^{f}$ is obtained for an incompressible flow with $\tilde{u}_{i}$ so defined. In some investigations, this term was incorporated with the macroscopic viscous stress tensor, thus forming a quantity for which a constitutive relation is formed (e.g., Hassanizadeh and Gray, 1980; Shapiro, 1981). Alternatively, it can be treated separately and a separate constitutive equation for $\left\langle\tilde{u}_{i} \tilde{u}_{j}\right\rangle^{f}$ can be assumed (e.g., Gray and O'Neill, 1976; Bear and Bachmat, 1986).

Based on the argument that the gradient of this term accounts for the mechanical dispersion of the momentum and it equals to zero when the fluid moves uniformly at the same velocity as the solid phase, Gray and O'Neill (1976) proposed the following constitutive equation

$$
<\tilde{u}_{i} \tilde{u}_{j}>^{f}=R_{i j}<u_{j}>^{f}
$$

for a rigid, stationary porous media. It is generally understood that the second order tensor $R_{i j}$ depends on the structure of the porous media and the pore velocity. A similar theoretical derivation of the dispersion flux was given by Bear and Bachmat (1986).

In addition to the dispersive term, closure must also be performed for the 
interfacial integral terms (see Equation (3.18)) which stand for the total forces exerted on the fluid by the solid within the REV and contain the information of the effects of porous microstructure and pore scale flow variables.

Slattery $(1969,1981)$ put two integrals, the pressure and the viscous integrals, in Equation (3.18) together and formed a general term. Based on the principle of material frame independence, this author assumed that the integral is a function of the difference between the local average fluid velocity and the local average solid velocity (the solid matrix may be undergoing a rigid body rotation and translation). For a rigid medium, this equation has been written in the vector form:

$$
\vec{F}=\mathbf{R}<\vec{u}>^{f}
$$

The resistance coefficient $\mathbf{R}$ in this case is a function of the magnitude of the local volume-average velocity of the fluid relative to the local volume-average velocity of the solid, a function of the viscosity of the fluid and the porosity, as well as a characteristic length $l_{c}$ of the porous media. Using the Buckingham-Pi theorem, Slattery at last arrived at an expression for $\mathbf{R}$ for an incompressible, Newtonian fluid through a nonoriented porous structure:

$$
\mathbf{R}=\frac{\phi \mu}{l_{c}^{2} K_{0}^{*}}
$$

where $K_{0}^{*}$ is dimensionless and is a function of the porosity only.

Following the work of Slattery (1969), many versions of the constitutive closure of the viscous integral have been presented either treating it together with the pressure integral or separately (Gray and O'Neill, 1976; Hassanazdh and Gray, 1980; Bear and Bachmat, 1986). One general assumption preserved in all the derivations is that the viscous integral is the intrinsic phase average of the viscous drag and this drag force is a function of the difference between solid and fluid intrinsic phase 
average velocities for Newtonian fluids. If there is no such relative motion, the net drag will be zero. According to this assumption, a macroscopic coefficient might then be formed in different ways and has to be determined experimentally.

In comparison, Whitaker (1986) (see also Quintard and Whitaker, 1988) presented a direct scheme without any constitutive assumptions to transform the closure problem into a boundary value problem of the deviation quantities for linear Stokes' flow.

\subsection{Remarks}

In concluding the literature review of this chapter, it may be stated that on one side, the phenomenological model at best provides a gross view of the correlations of the macroscopic properties for a specific porous medium, while on the other, although a more general and rigorous approach, the volumetric averaging method, is made, the application of this method still has some unsolved practical problems, such as the justification of the assumptions which have been used in the development of the theory and the quantification of the closure schemes. In other words, before pursuing any useful solutions, a quantitative analysis of the averaging theory based on the information at both microscopic and macroscopic scales must be performed.

Because of the extreme complexity of the porous media flow, a direct measurement of the microscopic quantities and the averaged quantities over an REV is an unattainable task at present, yet the high performance of computers and algorithms has stimulated the rapid growth of numerical flow simulations of complex geometric structures. It is possible to numerically analyze the motion at the microscopic scale to obtain the information on detailed flow mechanisms for a medium 
with different microstructures. At the same time, the volumetric average quantities may be extracted from the known distributions of the microscopic quantities by applying the averaging procedure. It is believed that this quantitative analysis of the relationship between the microscopic flow characteristics and the macroscopic transport phenomena will be a useful complement to other approaches in describing the physics of high $\mathrm{F}$-number flow. 


\section{CHAPTER 3}

\section{MACROSCOPIC DESCRIPTIONS OF POROUS MEDIA FLOW}

The objective of this chapter is to develop the mathematical concepts and relations that describe transport phenomena in porous media at the macroscopic level. A brief introduction to the volumetric averaging method which is the technique employed in the present study for achieving transition from the microscopic to the macroscopic level description is presented in Section 3.1. By applying the volumetric averaging method to the microscopic conservation equation, a general formulation and some special forms of the macroscopic momentum balance are

developed in Section 3.2. A new parameter, the Forchheimer number, is defined in Section 3.3 to serve as an indicator for the intensity of the nonlinearity in high F-number flow. By comparing the averaged momentum equations with a new form of the Forchheimer equation which is given in Section 3.3, the hydrodynamic definitions for the macroscopic coefficients are given in Section 3.4 in terms of the microscopic variables relevant to the transport process and the pore structure properties.

\subsection{Introduction to the Volumetric Averaging Method}

The volumetric averaging method, which is used in this study to derive the 
macroscopic conservation equations from the microscopic conservation equations, consists of associating a Representative Elementary Volume (REV) to every point inside the porous medium and averaging all the relevant transport variables and pore structure variables over it. The averaging is conducted throughout the domain of interest which results in a continuous and differentiable spatial distribution of the transport variables and the macroscopic properties of the pore structure (Whitaker, 1967, 1969; Gray, 1975; Gray and O'Neill, 1976; Hassanizadeh and Gary, 1979a, 1979b, 1980; Bachmat and Bear, 1986; Bear and Bachmat, 1986, 1991).

Because our primary interest is to analyze high $\mathrm{F}$-number flow by the volumetric averaging method, a conceptual model of a two phase system is introduced. The present analysis, therefore, is based upon the following conditions concerning the system:

- The solid matrix of porous material is nondeformable, stationary and consolidated.

- The Newtonian fluid consists of a single fluid phase with constant physical properties.

- The flow is laminar and steady.

- No mass exchange takes place between the fluid and solid phase.

For the system defined above, the geometric definitions of an REV used in the averaging procedure are schematically illustrated in Figure 3.1. The averaging volume $U_{b}$, which is contained in a surface $A_{b}$, consists of the volume occupied by the fluid $U_{f}$ and by the solid $U_{s}$, i.e., $U_{b}=U_{f} \cup U_{s}$. The boundary of $U_{f}$ consists of a material surface adjacent to the solid phase (and is denoted by $A_{f s}$ ), and a 


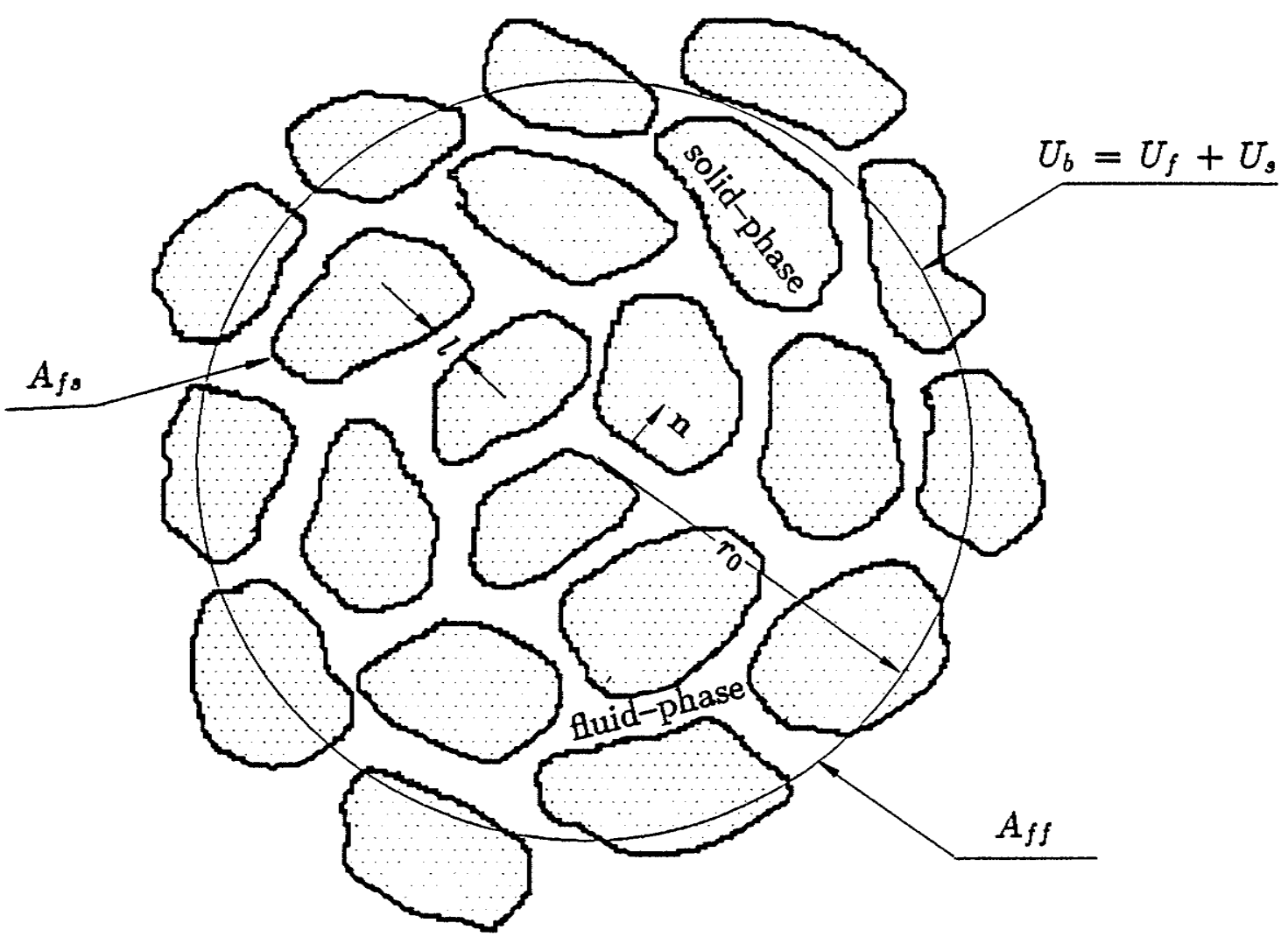

Fig. 3.1. A schematic averaging volume comprising a fluid phase and a solid phase. 
geometrical surface, $A_{f f}$, which is a part of the external boundary of $U_{b}$. The radius of the averaging volume is $r_{0}$ and the characteristic length for the fluid phase is denoted by $l$. In the following analysis, $\mathbf{r}$ denotes the microscopic position vector for the spatial point inside the averaging volume, and $\mathrm{X}$ denotes the macroscopic position vector for the spatial point defined as the centroid of an averaging volume.

The following set of definitions and averaging rules may be established for the system considered:

- A void distribution function is defined as

$$
\alpha(\mathbf{r})= \begin{cases}1, & \text { for } \mathbf{r} \in U_{f} \\ 0, & \text { for } \mathbf{r} \in U_{s}\end{cases}
$$

Thus, the local porosity, the void fraction of the averaging volume, is defined as

$$
\begin{aligned}
\phi(\mathbf{X}) & =\frac{1}{V_{b}} \int_{U_{b}} \alpha(\mathbf{r}) d U \\
& =\frac{1}{V_{b}} \int_{U_{f}} \alpha(\mathbf{r}) d U \\
& =\frac{V_{f}}{V_{b}}
\end{aligned}
$$

where $V_{f}$ and $V_{b}$ represent the volumetric values of the fluid phase and the bulk averaging volume, respectively.

- The volume average of a point quantity associated with the fluid phase, $\psi_{f}$, is

$$
<\psi_{f}>=\frac{1}{V_{b}} \int_{U_{b}} \psi_{f} \alpha(\mathrm{r}) d U
$$

which is often called the phase average of $\psi_{f}$ and is a function of the macroscopic position vector $\mathbf{X}$ only. The intrinsic phase average is defined as the volume average of the fluid property $\psi_{f}$ over the fluid phase:

$$
<\psi_{f}>^{f}=\frac{1}{V_{f}} \int_{U_{f}} \psi_{f} \alpha(\mathbf{r}) d U
$$


It follows from the definitions (3.3) and (3.4) that the two averages are related by the expression

$$
<\psi_{f}>=\phi<\psi_{f}>^{f}
$$

- In the averaging process, the average of the product, $<\psi_{f} \psi_{f}^{\prime}>^{f}$, must be replaced with the product of an average because an equation for $\left.<\psi_{f}\right\rangle^{f}$ is desired. A deviation term, the difference between the value of the microscopic quantity at point $\mathbf{r}$ within an REV which is centered at $\mathbf{X}_{0}$ and the corresponding intrinsic phase average over the REV is usually defined as:

$$
\left.\tilde{\psi}_{f}\right|_{\mathrm{r}}=\left.\psi_{f}\right|_{\mathrm{r}}-<\psi_{f}>\left.^{f}\right|_{\mathrm{X}_{0}}
$$

Accordingly, the average of the product may be written as

$$
<\psi_{f} \psi_{f}^{\prime}>^{f}=<\psi_{f}>^{f}<\psi_{f}^{\prime}>^{f}+<\tilde{\psi}_{f} \tilde{\psi}_{f}^{\prime}>^{f}
$$

that is, the intrinsic phase average of a product is equal to the sum of the product of the intrinsic phase average and the intrinsic phase average of the product of the deviations (Gray, 1975; Hassanizadeh and Gray, 1979a).

- In order to locally average the conservation equations, the average of a gradient must be replaced by the gradient of an average. These quantities are related by the averaging theorem (Slattery, 1967; Whitaker, 1967). For any tensorial property $\psi_{f}$ defined in the fluid phase, this theorem takes the form

$$
<\frac{\partial \psi_{f}}{\partial r_{i}}>=\frac{\partial<\psi_{f}>}{\partial X_{i}}+\frac{1}{V_{b}} \int_{A_{f s}} \psi_{f} \cos \left(\mathbf{n}, \mathbf{e}_{i}\right) d A
$$

where $A_{f s}$ represents the interphase area contained within the averaging volume, $\mathbf{n}$ is the unit normal vector pointing from the fluid phase to the solid phase, $\mathbf{e}_{i}$ is the unit vector in the direction of $r_{i}, r_{i}$ are the microscopic space 
coordinates and $X_{i}$ are the macroscopic space coordinates. The averaging theorem (3.8) may also be expressed in a modified form (Gray, 1975):

$$
\begin{aligned}
<\frac{\partial \psi_{f}}{\partial r_{i}}>^{f} & =\frac{\partial<\psi_{f}>^{f}}{\partial X_{i}}+\frac{1}{\phi V_{b}} \int_{A_{f_{s}}} \tilde{\psi}_{f} \cos \left(\mathbf{n}, \mathbf{e}_{i}\right) d A \\
& =\frac{\partial<\psi_{f}>^{f}}{\partial X_{i}}+\frac{1}{\phi V_{b}} \int_{A_{f_{s}}} \psi_{f} \cos \left(\mathbf{n}, \mathbf{e}_{i}\right) d A+\frac{<\psi_{f}>^{f}}{\phi} \frac{\partial \phi}{\partial X_{i}} .
\end{aligned}
$$

- The average at any given point $\mathbf{X}$ within the REV can be expressed in terms of the average at the centroid $\mathbf{X}_{0}$ linearly:

$$
<\psi_{f}>\left.^{f}\right|_{\mathbf{X}}=<\psi_{f}>\left.^{f}\right|_{\mathbf{X}_{0}}+\left.\left(\nabla<\psi_{f}>^{f}\right)\right|_{\mathbf{X}_{0}}\left(\mathbf{X}-\mathbf{X}_{0}\right) .
$$

The introduction of assumption (3.10) will greatly simplify the theoretical treatment. Without justification, another phenomenological assumption is that the length scale associated with averaged quantities is the macroscopic length scale $l_{m a c}$ which is much larger than the microscopic scale. These assumptions about the macroscopic quantity have been often employed in simplifying the macroscopic conservation equations (Whitaker, 1969, 1986; Hassnnizadeh and Gray, 1987; Bear and Bachmat, 1986).

In principle, the averaging rules described above may be applied over any size of volume. However, to achieve a single valued and continuously differentiable property field at the macroscopic level, only a range of averaging volume sizes gives meaningful statistic averages for a specific porous medium. An averaging volume which belongs to that range is what has been referred to as the Representative Elementary Volume (REV). Traditionally, the constraints for the characteristic length of an REV are expressed by

$$
l<<r_{0}<<l_{\text {mac }}
$$

where $l_{m a c}$ is again a characteristic length identifying the macroscopic scale. 


\subsection{Macroscopic Momentum Equations}

In this section, the basic averaging rules listed in Section 3.1 will be applied to derive the macroscopic momentum equation governing the mechanical phenomena in porous media. The physical meaning of different terms of this equation will be explained, and several simplified forms of the general governing equation which are of interest in this research will be briefly discussed.

\subsubsection{Momentum Equation for Heterogeneous Media}

This study is concerned with a single, incompressible, Newtonian fluid with constant viscosity, flowing steadily through a rigid porous medium. The linear momentum equation governing the microscopic flow process is:

$$
\underbrace{\rho \frac{\partial\left(u_{i} u_{j}\right)}{\partial r_{j}}}_{\text {Inertial }}+\underbrace{\frac{\partial p}{\partial r_{i}}}_{\text {Pressure }}-\underbrace{\mu \frac{\partial^{2} u_{i}}{\partial r_{j} \partial r_{j}}}_{\text {Viscous }}-\underbrace{\rho a_{i}}_{\text {Body }}=0 .
$$

In this equation, $u_{k}$ is the microscopic velocity, $r_{k}$ is the microscopic space coordinate, $p$ is the microscopic pressure and $a_{i}$ is the body force acceleration. As underlined, the different terms in Equation (3.12) represent, respectively, the inertial effect, the pressure force, the viscous force and the body force (per unit volume) at a point in the microscopic flow. These terms will be in balance at every point of the flow field. However, the different terms, which represent different physical phenomena, play changing rôles in different flow and geometry conditions. A knowledge of how these terms change for different conditions aids in the understanding of the nature of flow in a porous medium. 
Equation (3.12) can be averaged over an REV:

$$
<\rho \frac{\partial\left(u_{i} u_{j}\right)}{\partial r_{j}}>^{f}+<\frac{\partial p}{\partial r_{i}}>^{f}-<\mu \frac{\partial^{2} u_{i}}{\partial r_{j} \partial r_{j}}>^{f}-<\rho a_{i}>^{f}=0 .
$$

According to the averaging rules, the intrinsic phase average of the different terms in Equation (3.13) can be expressed in the following form:

The pressure term:

$$
<\frac{\partial p}{\partial r_{i}}>^{f}=\frac{\partial<p>^{f}}{\partial X_{i}}+\frac{1}{\phi V_{b}} \int_{A_{f s}} p \cos \left(\mathbf{n}, \mathbf{e}_{i}\right) d A+\frac{<p>^{f}}{\phi} \frac{\partial \phi}{\partial X_{i}}
$$

The inertial term:

$$
\begin{aligned}
\rho<\frac{\partial\left(u_{i} u_{j}\right)}{\partial r_{j}}>^{f}= & \rho \frac{\partial<u_{i} u_{j}>^{f}}{\partial X_{j}}+\frac{\rho<u_{i} u_{j}>^{f}}{\phi} \frac{\partial \phi}{\partial X_{j}} \\
= & \rho \frac{\partial\left(<u_{i}>^{f}<u_{j}>^{f}\right)}{\partial X_{j}}+\rho \frac{\partial<\tilde{u}_{i} \tilde{u}_{j}>^{f}}{\partial X_{j}} \\
& +\frac{\rho<u_{i} u_{j}>^{f}}{\phi} \frac{\partial \phi}{\partial X_{j}},
\end{aligned}
$$

The viscous term*:

$$
\begin{aligned}
\mu<\frac{\partial^{2} u_{i}}{\partial r_{j} \partial r_{j}}>^{f}= & \mu<\frac{\partial}{\partial r_{j}}\left(\frac{\partial u_{i}}{\partial r_{j}}\right)>^{f} \\
= & \mu \frac{\partial^{2}<u_{i}>^{f}}{\partial X_{j} \partial X_{j}}+\frac{\mu}{\phi V_{b}} \int_{A_{f o}}\left(\frac{\partial u_{i}}{\partial r_{j}}\right) \cos \left(\mathbf{n}, \mathbf{e}_{j}\right) d A \\
& +\frac{2 \mu}{\phi} \frac{\partial<u_{i}>^{f}}{\partial X_{j}} \frac{\partial \phi}{\partial X_{j}}+\frac{\mu<u_{i}>^{f}}{\phi} \frac{\partial^{2} \phi}{\partial X_{j} \partial X_{j}},
\end{aligned}
$$

The body force term

$$
\begin{aligned}
<\rho a_{i}>^{f} & =\rho<a_{i}>^{f} \\
& =\rho a_{i},
\end{aligned}
$$

\footnotetext{
* A derivation of the averaging of the microscopic viscous term is given in APPENDIX A.
} 
Substituting the expressions (3.14), (3.15), (3.16) and (3.17) into Equation (3.13), the final form of the averaged momentum equation can be obtained:

$$
\begin{aligned}
& \rho \frac{\partial\left(<u_{i}>^{f}<u_{j}>^{f}\right)}{\partial X_{j}}+\rho \frac{\partial<\tilde{u}_{i} \tilde{u}_{j}>^{f}}{\partial X_{j}}+\frac{\partial<p>^{f}}{\partial X_{i}}-\mu \frac{\partial^{2}<u_{i}>^{f}}{\partial X_{j} \partial X_{j}}-\rho a_{i} \\
& +\frac{1}{\phi V_{b}} \int_{A_{f s}} p \cos \left(\mathbf{n}, \mathbf{e}_{i}\right) d A \\
& -\frac{\mu}{\phi V_{b}} \int_{A_{f a}}\left(\frac{\partial u_{i}}{\partial r_{j}}\right) \cos \left(\mathbf{n}, \mathbf{e}_{j}\right) d A \\
& +\frac{<p>^{f}}{\phi} \frac{\partial \phi}{\partial X_{i}} \\
& +\left[\rho<u_{i} u_{j}>^{f}-2 \mu \frac{\partial<u_{i}>^{f}}{\partial X_{j}}\right] \frac{1}{\phi} \frac{\partial \phi}{\partial X_{j}} \\
& -\mu \frac{<u_{i}>^{f}}{\phi} \frac{\partial^{2} \phi}{\partial X_{j} \partial X_{j}}=0 .
\end{aligned}
$$

Different from the previous published derivations, the terms of the porosity gradient which are related to the effects of the heterogeneity of the porous medium have been separated in this equation. Equation (3.18) represents a macroscopic momentum balance for flow through porous media. Similar to the microscopic momentum equation (Equation (3.12)), the first five terms account for the inertial effect, pressure force, viscous force and body force on the macroscopic scale. The second term is usually called the dispersion term*. The two integral terms which result from the averaging process contain the information on how the microstructure of the porous medium affects the traversing fluid phase, and their values are dominated by the local geometry and flow conditions. The last three terms in Equation (3.18) are associated with the heterogeneity of the porous media.

It is of some importance to note that the integral term which arises from the averaging of the microscopic inertial term (Equation (3.15)) always vanishes because of the application of the no-slip condition on the fluid/solid interface $A_{f s}$.

\footnotetext{
* see APPENDIX B for a detailed analysis for the dispersion term.
} 
This has misled some researchers to conclude that the microscopic inertial effect is irresponsible for the nonlinearity in high $\mathrm{F}$-number flow.

A general solution of practical interest for Equation (3.18) is still far beyond our ability. An explanation of the remaining problems and the possible approaches to solve them are given in Chapter 9 .

\subsubsection{Momentum Equation for Homogeneous Media}

To center our attention on the understanding of the microscopic inertial effects, the general momentum equation (3.18) may be simplified for the case of macroscopically uniform flow through a homogeneous porous medium. Under these conditions, the macroscopic inertial term (except the dispersion term), viscous term and heterogeneous terms vanish, and Equation (3.18) takes the form

$$
\begin{aligned}
-\frac{\partial<p>^{f}}{\partial X_{i}}= & \rho \frac{\partial<\tilde{u}_{i} \tilde{u}_{j}>^{f}}{\partial X_{j}}-\rho a_{i} \\
& +\frac{1}{\phi V_{b}} \int_{A_{f s}} p \cos \left(\mathbf{n}, \mathbf{e}_{i}\right) d A-\frac{\mu}{\phi V_{b}} \int_{A_{f s}}\left(\frac{\partial u_{i}}{\partial r_{j}}\right) \cos \left(\mathbf{n}, \mathbf{e}_{j}\right) d A .
\end{aligned}
$$

It is evident that to go further for the solution of Equation (3.19), the velocity and the pressure fields on the pore scale are needed in evaluation of the integral terms. The computations of the microscopic flow fields will be the topic of the next chapter.

\subsubsection{Momentum Equation for a Periodic Diverging- Converging Capillary Model}

In actual porous media, the configuration of the flow channels is highly irregular. The influence of pore-structure on the traversing flow may apparently be attributed 
to several effects, such as diverging-converging, channel axis curvature, and flow branching. In the present research, a simplified porous media model, the periodic diverging-converging capillary tube (see Figure 3.2), has been chosen to allow the numerical simulation of the effects of the sudden change in the cross-sectional area, an important characteristic of the microstructure. A similar model has been utilized by several researchers (e.g., Payatakes et al., 1973; Dullien and Azzam, 1973; Azzam and Dullien, 1977; Coulaud et al., 1988). The present approach, however, mainly emphasizes the numerical analysis of the relationship between macroscopic and microscopic phenomena in high $\mathrm{F}$-number flow, based on the continuum approach.

Consideration is given to the spatially periodic medium (Figure 3.2) which is generated by the repetition of the diverging-converging unit cell. Due to the periodic fully developed feature (Patankar et al., 1977), only one period of the identical segments called the Representative Unit Cell (RUC) is needed in the flow calculations.

Let $(x, r)$ be the microscopic cylindrical coordinates, $X$ be the only macroscopic coordinate in the present case and be oriented horizontally in the $x$ direction, and $(\mathbf{i}, \mathbf{j})$ and $(u, v)$ be the unit vectors and the microscopic velocities in the $(x, r)$ directions respectively. Other parameters characterizing the domain of the solution are defined in Figure 3.3.

For the geometric model described above, the averaged momentum equation (3.19) may be written as:

$$
-\frac{d<p>^{f}}{d X}=-\frac{\mu}{\phi V_{b}} \int_{A_{f o}}\left(\frac{\partial u}{\partial r}\right) \cos (\mathbf{n}, \mathbf{j}) d A+\frac{1}{\phi V_{b}} \int_{A_{f s}} p \cos (\mathbf{n}, \mathbf{i}) d A .
$$

In deriving Equation (3.20) from Equation (3.19), the dispersion term 


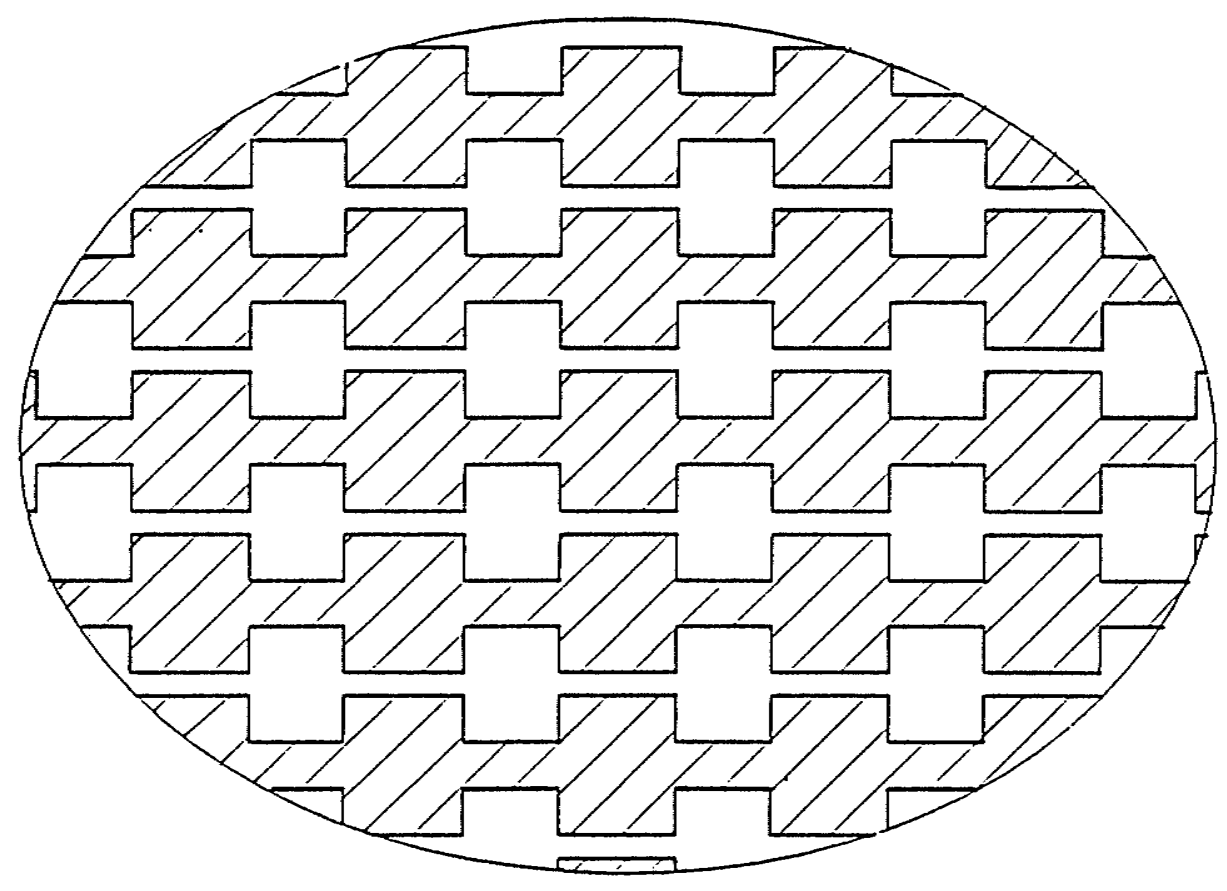

Fig. 3.2. The periodic diverging-converging capillary model. 


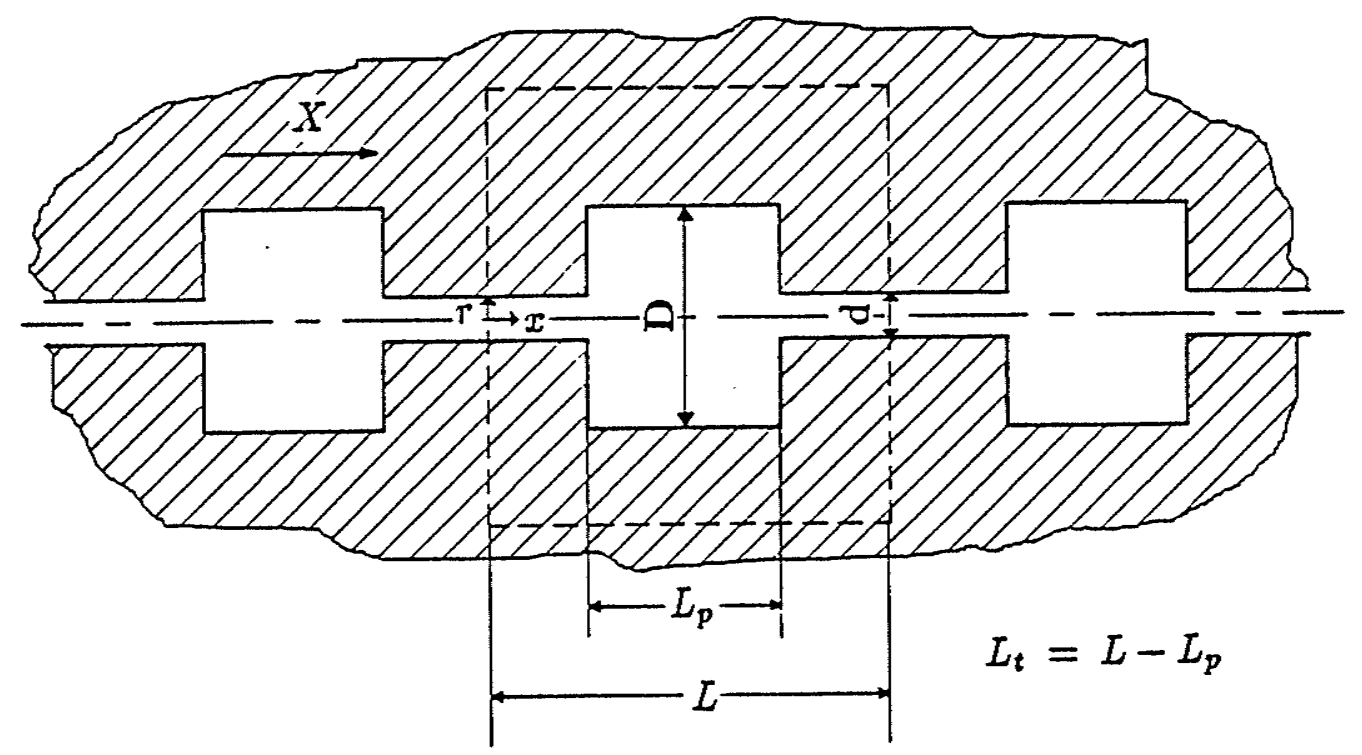

Fig. 3.3. The schematic of a periodic diverging-converging representative unit cell. 
$\rho \frac{d<\tilde{u}_{i} \tilde{u}>^{f}}{d X}$ in this case becomes zero due to the application of the periodically fully developed flow condition*. The body force term has been ignored for simplicity although it can be conveniently combined with the pressure term on the left hand side of Equation (3.20) to form a hydraulic potential.

As shown in APPENDIX C, for this model the macroscopic pressure gradient in Equation (3.20) can also be expressed in the form of surface integrals over the entrance and exit surface of the diverging-converging RUC:

$$
\frac{d<p>^{f}}{d X}=\frac{1}{\phi V_{b}} \int_{A_{f f}} p \cos (\mathbf{n}, \mathbf{i}) d A=\frac{\xi}{\phi}\left(\frac{\bar{P}_{e x}-\bar{P}_{e n}}{L}\right)
$$

where $A_{f f}$ is the area of the fluid phase intersection with the averaging volume surface (the area of entrance and exit of the unit cell), $\xi$ is the areosity at the entrance and exit cross-section, defined as the ratio of $A_{f}$, the area open for flow, to $A_{b}$, a fictitious area which numerically equals the quotient of the total crosssectional area of the medium and the number of the parallel flow channels, and $\bar{P}_{e n}, \bar{P}_{e x}$ are the mean pressures on the entrance and the exit.

Applying Equation (3.21), the momentum governing equation for the periodic diverging-converging capillary model can be written as

$$
-\left(\frac{\bar{P}_{e x}-\bar{P}_{e n}}{L}\right)=-\frac{\mu}{\xi V_{b}} \int_{A_{f_{s}}}\left(\frac{\partial u}{\partial r}\right) \cos (\mathbf{n}, \mathbf{j}) d A+\frac{1}{\xi V_{b}} \int_{A_{f_{s}}} p \cos (\mathbf{n}, \mathbf{i}) d A .
$$

It is interesting to note that Equation (3.22) is nothing but the integral momentum equation found by taking the RUC as the control volume.

For flow in a straight cylindrical capillary $(D / d=1)$, the pressure integral in Equation (3.22) equals zero. A simple calculation gives immediately the relationship between the pressure and the mean velocity in Hagen-Poiseuille flow (see,

\footnotetext{
* See APPENDIX B for the proof of this argument.
} 
e.g., Fox and McDonald, 1985)

$$
-\left(\frac{\bar{P}_{e x}-\bar{P}_{e n}}{L}\right)=\frac{32}{d^{2}} \mu \bar{U}_{d} .
$$

where $\bar{U}_{d}$ is the mean velocity in the small pipe of the capillary.

\subsubsection{Expressions for the Interfacial Drag Force}

For the averaged momentum equations derived in the previous subsections, a viscous surface integral term

$$
V_{I N T}=-\frac{\mu}{\phi V_{b}} \int_{A_{f s}}\left(\frac{\partial u_{i}}{\partial r_{j}}\right) \cos \left(\mathbf{n}, \mathbf{e}_{j}\right) d A
$$

and a pressure surface integral term

$$
P_{I N T}=\frac{1}{\phi V_{b}} \int_{A_{f s}} p \cos \left(\mathbf{n}, \mathbf{e}_{i}\right) d A
$$

are contained in the macroscopic fluid transport equations. Both $V_{I N T}$ and $P_{I N T}$ are macroscopic quantities, and these two terms represent, physically, the drag force exerted by the solid matrix on the flowing fluid at their contact surfaces within an REV, per unit volume of the fluid phase. To achieve any solutions of the averaged momentum equation, these integrals, which characterize the particularity of a specific system, must be first effectively modelled and quantitatively determined for the given problem. Many versions of the constitutive closure for these integral terms have been reviewed in Chapter 2. In the present study, instead of deriving theoretical closure expressions, the pressure and the viscous integrals have been determined numerically for the periodic model (Figure 3.3). In this case, the two surface integrals take the form

$$
V_{I N T}=-\frac{\mu}{\phi V_{b}} \int_{A_{f_{s}}}\left(\frac{\partial u}{\partial r}\right) \cos (\mathbf{n}, \mathbf{j}) d A
$$


and

$$
P_{I N T}=\frac{1}{\phi V_{b}} \int_{A_{f a}} p \cos (\mathbf{n}, \mathbf{i}) d A
$$

If the pore scale variables are known, the integral terms may be calculated numerically at various combinations of micro-geometry and flow rate in a systematic way. The data will help to improve the rationality of the constitutive simulation of the interfacial drag force.

\subsection{The Forchheimer Number}

Macroscopically, the porous media flow is well described by the Forchheimer equation (2.2) at higher filtration velocities. Before seeking the answer for how the microscopic phenomena affect the bulk quantities by performing a comparison with the averaging procedure presented in the last subsection, a new form of Forchheimer equation will be proposed, proceeding from the consideration that the permeability may be treated as being velocity dependent. A new dimensionless parameter, the Forchheimer number, is introduced to serve as a criterion to indicate when the nonlinear effects become nonnegligible (Ruth and $\mathrm{Ma}, 1992)$.

As mentioned by Scheidegger (1960), the determination of the upper range of validity of Darcy's law has presented an uncertainty in the medium's critical Reynolds number by a factor of 750 . The reason for this has been mainly attributed to the actual indeterminacy of the geometric characteristic length. The following results will show that the Reynolds number, which is well defined in situations of pipe-like flow, is not an appropriate parameter to describe high $\mathrm{F}$-number flow. 
To do so, the conventional Forchheimer equation is rearranged in the form:

$$
\begin{aligned}
-\frac{d \bar{P}}{d X} & =\frac{\mu}{k} \bar{U}+\beta \rho \bar{U}^{2} \\
& =\frac{1}{k}\left(1+\frac{\beta k \rho \bar{U}}{\mu}\right) \mu \bar{U} \\
& =\frac{1}{k}(1+F o) \mu \bar{U} \\
& =\frac{\mu}{k_{v}} \bar{U}
\end{aligned}
$$

where $k_{v}=k /\left(1+F_{o}\right)$ is the velocity-dependent permeability and $F o$, as defined in Equation (3.28), is called the Forchheimer number. Equation (3.28) suggests that the permeability may alternatively be treated as a velocity dependent parameter for high $\mathrm{F}$-number flow and the Forchheimer number Fo really works as a dimensionless criterion to indicate when microscopic effects lead to significant macroscopic nonlinear effects.

The definition of the Forchheimer number may be treated so as to contain a Reynolds number explicitly

$$
F_{0}=\frac{\beta k \rho \bar{U}}{\mu}=\beta \frac{k}{D_{c}} \frac{\rho \bar{U} D_{c}}{\mu}=\beta \frac{k}{D_{c}} R e,
$$

where $D_{c}$ is some characteristic length scale of a porous medium. In comparison with Equation (3.28), it is evident that the macroscopic nonlinear effects depend on both the macroscopic flow rate (the 'medium Reynolds number') and the microstructure of the medium because $\beta$ is structure dependent, and the Forchheimer number has taken both factors into account. Therefore, to characterize the nonlinear effects in porous media flow, the terms 'high velocity flow' or ' high Reynolds number flow' are misleading. The best term is probably 'high Forchheimer number flow'. Because of its structure dependence, on the other hand, the determination of the Forchheimer number needs a detailed knowledge of the microscopic flow. 


\subsection{Hydrodynamic Definitions of Macroscopic Coefficients}

In this section, more precise definitions will first be derived for the macroscopic coefficients in the Forchheimer equation by comparing Equation (3.28) with the averaged momentum equation (3.22). For this purpose, the integrals on the right hand side of Equation (3.22) are reorganized such that they are expressed in terms of filtration velocity $\bar{U}$. This can be achieved by making the terms dimensionless by using $d$, the diameter of the narrow pipe (Figure 3.3 ) as the length scale, $\bar{U}_{d}$, the average velocity in this pipe as the velocity scale, and $\rho \bar{U}_{d}^{2}$ as the pressure scale. Equation (3.22) can then be written as

$$
\begin{aligned}
-\left(\frac{\bar{P}_{e x}-\bar{P}_{e n}}{L}\right)= & {\left[-\frac{1}{d^{2} \xi A_{f}^{*} L^{*}} \int_{A_{f o}^{*}}\left(\frac{\partial u^{*}}{\partial r^{*}}\right) \cos (\mathbf{n}, \mathbf{j}) d A^{*}\right] \mu \bar{U} } \\
& +\left[\frac{1}{d \xi^{2} A_{f}^{*} L^{*}} \int_{A_{f o}^{*}} p^{*} \cos (\mathbf{n}, \mathbf{i}) d A^{*}\right] \rho \bar{U}^{2}
\end{aligned}
$$

where

$$
u^{*}=\frac{u}{\bar{U}_{d}} ; \quad p^{*}=\frac{p}{\rho \bar{U}_{d}^{2}} ; \quad A_{f}^{*}=\frac{A_{f}}{d^{2}} ; \quad A_{f s}^{*}=\frac{A_{f s}}{d^{2}} ; \quad L^{*}=\frac{L}{d} ; \quad r^{*}=\frac{r}{d} .
$$

A conceptually similar expression has been derived by Dullien and Azzam (1973). In comparison with the empirical Forchheimer equation (2.2), they straightforwardly concluded that the bracketed term in front of $\bar{U}$ was the reciprocal permeability and the other bracketed expression, in front of $\widetilde{U}^{2}$, was the inertial coefficient $\beta$.

In the range of validity of the Forchheimer equation, $k$ and $\beta$ are constants. The permeability $k$ is by physical definition the limiting value at very low flow rate and must be mathematically defined as such.

In the following, the definitions of $k$ and $\beta$ will be given. However, it must be kept in mind that although the Forchheimer equation seems to be in good 
agreement with experimental evidence, it is not a unique expression to treat the dependence of pressure drop and filtration velocity.

The pressure integral on the right hand side of Equation (3.30) can be rearranged so as to contain a Reynolds number explicitly:

$$
\begin{aligned}
& {\left[\frac{1}{d \xi^{2} A_{f}^{*} L^{*}} \int_{A_{f_{0}^{*}}^{*}} p^{*} \cos (\mathbf{n}, \mathbf{i}) d A^{*}\right] \rho \bar{U}^{2}} \\
& =\left(\frac{1}{d^{2} \xi}\right)\left[\frac{1}{A_{f}^{*} L^{*}} \int_{A_{f_{o}}^{*}} p^{*} \cos (\mathbf{n}, \mathbf{i}) d A^{*}\right]\left(\frac{\rho \bar{U}_{d} d}{\mu}\right) \mu \bar{U} \\
& =\left(\frac{1}{d^{2} \xi}\right)\left[\frac{1}{A_{f}^{*} L^{*}} \int_{A_{f_{o}}^{*}} p^{*} \cos (\mathbf{n}, \mathbf{i}) d A^{*}\right]\left(R_{d}\right) \mu \bar{U} \\
& =\left(\frac{1}{d^{2} \xi}\right)(B) R e_{d} \mu \bar{U}
\end{aligned}
$$

where

$$
B=\frac{1}{A_{f}^{*} L^{*}} \int_{A_{f_{s}^{*}}^{*}} p^{*} \cos (\mathbf{n}, \mathbf{i}) d A^{*}
$$

and the Reynolds number

$$
R e_{d}=\frac{\rho \bar{U}_{d} d}{\mu}
$$

is based on the mean velocity $\bar{U}_{d}$ in the narrow pipe and the diameter of that pipe, $d$.

Letting $A$ to represent the viscous integral of Equation (3.30):

$$
A=-\frac{1}{A_{f}^{*} L^{*}} \int_{A_{f_{\theta}}^{*}}\left(\frac{\partial u^{*}}{\partial r^{*}}\right) \cos (\mathbf{n}, \mathbf{j}) d A^{*}
$$

Equation (3.30) may be written as:

$$
-\left(\frac{\bar{P}_{e x}-\bar{P}_{e n}}{L}\right)=\frac{1}{d^{2} \xi}\left(A+B R e_{d}\right) \mu \bar{U} .
$$

By comparing with Equation (3.28), it can be seen that the permeability $k_{v}$ is indeed velocity dependent. The simplest way to treat this velocity dependence is 
by a linear model, and the Forchheimer equation arises as a simple, but nonunique, formulation for flow in porous media.

By making a comparison between Equation (3.35) and Equation (3.28), the following hydraulic definitions containing $A, B$ can be obtained :

$$
\begin{gathered}
k=\frac{d^{2} \xi}{\left[A+B R e_{d}\right]_{R e_{d} \rightarrow 0}} \\
\beta=\left(\frac{1}{d \xi^{2}}\right) \frac{\left[A+B R e_{d}\right]-\left[A+B R e_{d}\right]_{R e_{d} \rightarrow 0}}{R e_{d}} \\
F_{o}=\frac{\left[A+B R e_{d}\right]-\left[A+B R e_{d}\right]_{R e_{d} \rightarrow 0}}{\left[A+B R e_{d}\right]_{R e_{d} \rightarrow 0}}
\end{gathered}
$$

A knowledge of how these porous media parameters change at various combinations of pore geometry and flow rate will help in the understanding of the mechanism of transport phenomena.

For computational purposes, Equation (3.35) may also be expressed in dimensionless form

$$
-\left(\frac{\bar{P}_{e x}^{*}-\bar{P}_{e n}^{*}}{L^{*}}\right)=-\left(\frac{\Delta \bar{P}^{*}}{L^{*}}\right)=\frac{\left(A+B R e_{d}\right)}{R e_{d}}
$$

It should be emphasized that the product term, $B R e_{d}$, will keep a finite, non-zero value as $R e_{d} \rightarrow 0$ because the pressure integral $B \rightarrow \infty$ in that case. This implies that the permeability $k$ should be defined such that the effects of the interfacial pressure force have been taken into account.

Following a similar procedure, it will not be difficult to derive the general expressions for the macroscopic coefficients in more complicated cases of heterogeneous media. The real difficulty in obtaining the quantitative descriptions of the phenomena lies in the accurate predictions of the microscopic flow fields, even for the highly simplified diverging-converging capillary model. 


\section{CHAPTER 4}

\section{MATHEMATICAL MODEL FOR}

\section{MICROSCOPIC FLOW ANALYSIS}

The macroscopic momentum equations and the expressions for the macroscopic coefficients have been derived in Chapter 3 through the volumetric averaging method, and the problems involved in the further solution process have also been stated explicitly.

As already shown by the earlier chapters, the volumetric average treatment has bridged the gap between the macroscopic and the microscopic phenomena. The accurate prediction of the properties for porous media flow through the continuum approach are now dependent entirely on a knowledge of microscopic flow mechanisms. In the present chapter, attention will be turned to the quantitative analysis of the microscopic level flow. To begin with, the geometry of the solution domain for the postulated diverging-converging capillary model will be given in

Section 4.1. Later, the governing differential equations and the relevant boundary conditions which form a complete mathematical model for the analysis of pore space flow will be considered in Sections 4.2 and 4.3 .

\subsection{Geometry of the Solution Domain}

The periodic diverging-converging capillary model has been selected in this research to accomplish the numerical simulation of the flow within the microstruc- 
ture. Geometrically, the model can be thought to be generated by connecting in series an infinite number of cylindrical segments of different sizes(Figure 3.2). Therefore, one important feature of the pore space of real porous media-sudden changes in a flow channel-is accommodated by this model. Because of the periodic fully developed nature (see Subsection 4.3.2 for more details), only one period of the identical segments (one wavelength) is needed for the numerical solutions. In other words, the representative elementary volume (REV) is in turn represented by a cubic representative unit cell (RUC) as shown in Figure 4.1, and all the average geometrical properties of the REV can be embedded within this RUC. The axial cross-section of an RUC which forms the geometry of the computational domain is illustrated in Figure 4.2. It is apparent that four linear parameters will determine the domain completely. They are the diameter and length of the small pipe, $d$ and $L_{t}$, and the diameter and length of the large pipe, $D$ and $L_{p}$. In theory, any segment with a wavelength $L$ between two cross-sections can be used as an RUC. However, it will be seen in Chapter 6 that the present RUC is the most appropriate for the purpose of accurate numerical simulations.

\subsection{Governing Equations for Microscopic Flow}

For the diverging-converging RUC described above, the steady, axisymmetrical, incompressible flow of a Newtonian fluid with no body force and constant properties is governed by the Navier-Stokes equation, appropriately simplified. In the cylindrical coordinate system $(x, r, \theta)$ shown in Figure 4.2 , the flow is two dimensional, and the axial and radial components of the momentum equation (3.12) can be written in the dimensionless form: 


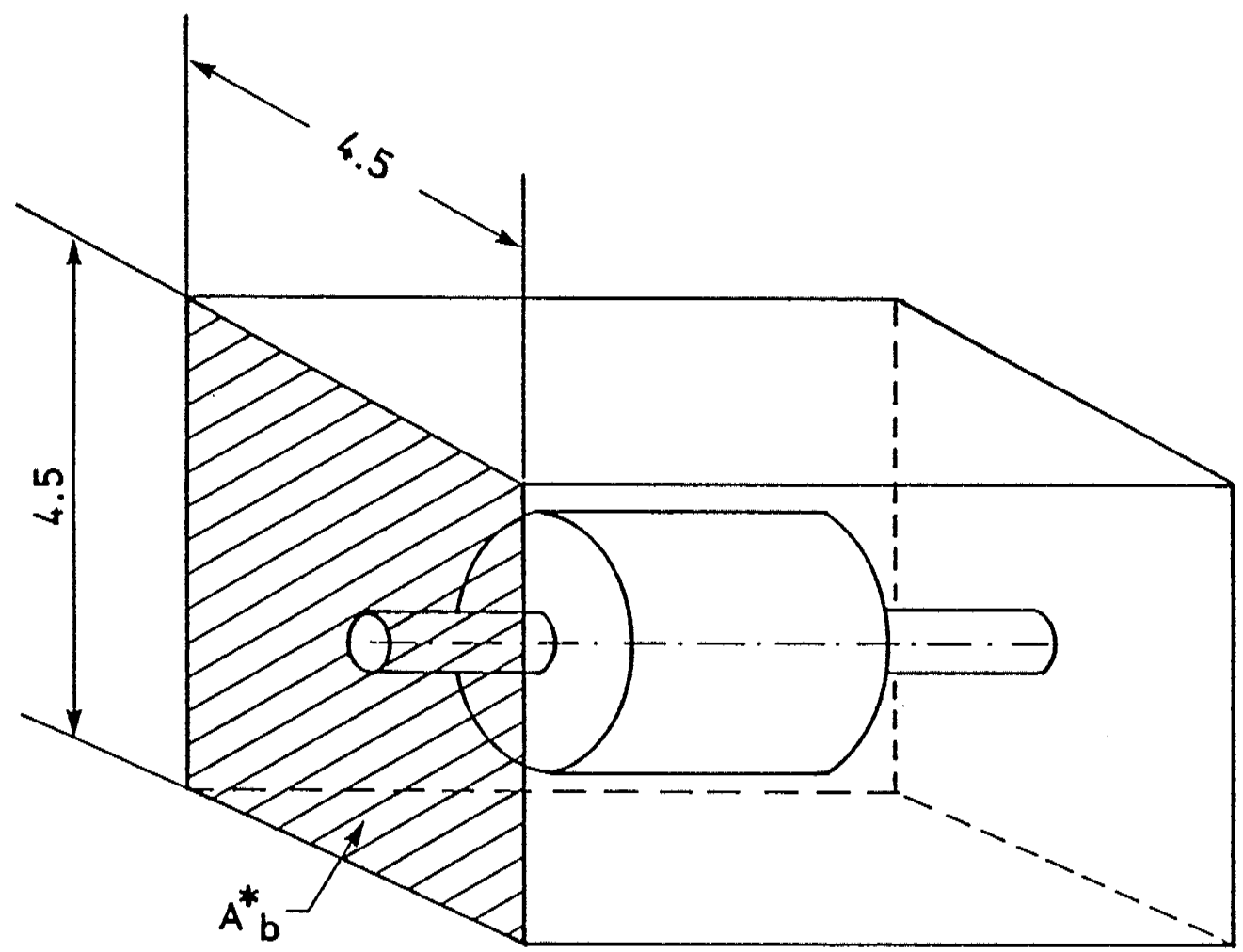

Fig. 4.1. A representative unit cell (RUC). 


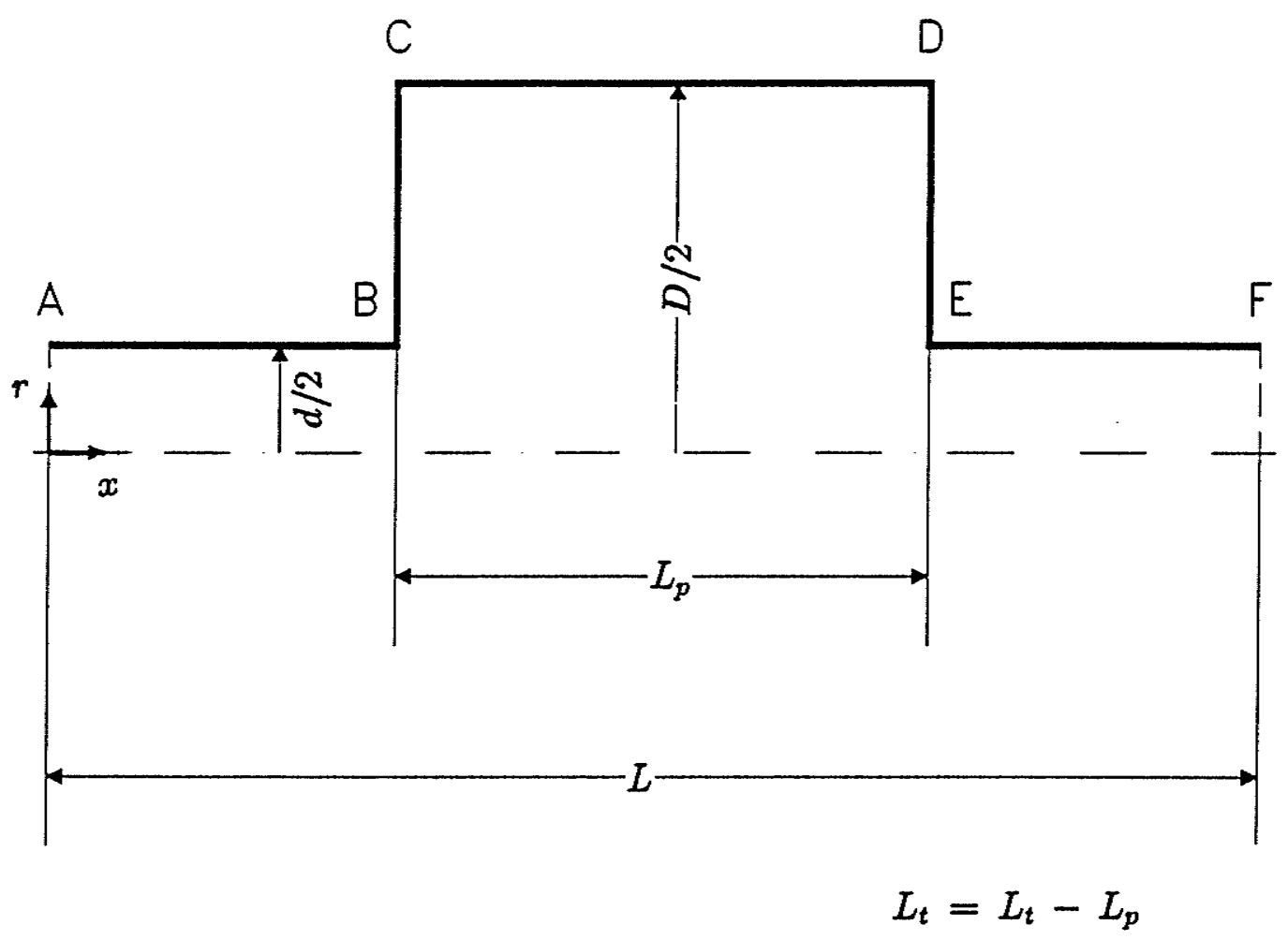

Fig. 4.2. Geometry of the solution domain. 
Axial component:

$$
-\frac{\partial p^{*}}{\partial x^{*}}+\frac{1}{R e_{d}}\left[\frac{\partial^{2} u^{*}}{\partial x^{* 2}}+\frac{1}{r^{*}} \frac{\partial}{\partial r^{*}}\left(r^{*} \frac{\partial u^{*}}{\partial r^{*}}\right)\right]-\left(u^{*} \frac{\partial u^{*}}{\partial x^{*}}+v^{*} \frac{\partial u^{*}}{\partial r^{*}}\right)=0
$$

\section{Radial component:}

$$
-\frac{\partial p^{*}}{\partial r^{*}}+\frac{1}{R e_{d}}\left[\frac{\partial^{2} v^{*}}{\partial x^{* 2}}+\frac{1}{r^{*}} \frac{\partial}{\partial r^{*}}\left(r^{*} \frac{\partial v^{*}}{\partial r^{*}}\right)-\frac{v^{*}}{r^{* 2}}\right]-\left(u^{*} \frac{\partial v^{*}}{\partial x^{*}}+v^{*} \frac{\partial v^{*}}{\partial r^{*}}\right)=0
$$

In this coordinate system, the equation of continuity is

$$
\frac{1}{r^{*}} \frac{\partial\left(r^{*} v^{*}\right)}{\partial r^{*}}+\frac{\partial u^{*}}{\partial x^{*}}=0
$$

where $\left(u^{*}, v^{*}\right)$ are the dimensionless velocities in the $\left(x^{*}, r^{*}\right)$ coordinate directions, $R e_{d}=\rho \bar{U}_{d} d / \mu$ is the Reynolds number, $\rho$ is the density, $\mu$ is the dynamic viscosity, $\bar{U}_{d}$ is the mean velocity in the small diameter pipe (the throat), and $d$ is the diameter of this pipe. Similar to the treatment in Section 3.4, the equations have been made dimensionless by using $d$ as the length scale, $\bar{U}_{d}$ as the velocity scale, and $\rho \bar{U}_{d}^{2}$ as the pressure scale.

Equations (4.1) to (4.3) may be solved simultaneously to provide a solution for $u^{*}, v^{*}$ and $p^{*}$. However, an alternative is to utilize a vorticity-stream function approach.

\subsubsection{Vorticity-Stream Function Approach}

The coupled nonlinear partial differential equations (4.1) and (4.2) contain pressure terms in the gradient form. In most cases, the pressure distribution is unknown. Therefore, it is advantageous to remove the explicit pressure terms from these equations. For this purpose, the vorticity transport equation may be obtained by 
cross-differentiating Equation (4.1) with respect to $r^{*}$ and Equation (4.2) with respect to $x^{*}$ and eliminating the pressure terms:

$$
\begin{aligned}
\left(\frac{\partial^{2} \Omega^{*}}{\partial x^{* 2}}+\frac{\partial^{2} \Omega^{*}}{\partial r^{* 2}}\right) & -R e_{d}\left[u^{*} \frac{\partial \Omega^{*}}{\partial x^{*}}+\left(v^{*}-\frac{1}{R e_{d}} \frac{1}{r^{*}}\right) \frac{\partial \Omega^{*}}{\partial r^{*}}\right]+ \\
& +\operatorname{Re}_{d}\left[\Omega^{*}\left(\frac{v^{*}}{r^{*}}-\frac{1}{R e_{d}} \frac{1}{r^{* 2}}\right)\right]=0
\end{aligned}
$$

In this equation, $\Omega^{*}$ represents vorticity which is defined as the curl of the velocity vector, and, in the present case, the only nonvanishing component of the vorticity is

$$
\Omega^{*}=\frac{\partial v^{*}}{\partial x^{*}}-\frac{\partial u^{*}}{\partial r^{*}}
$$

By introducing the definition of the stream function $\left(\psi^{*}\right)$ :

$$
u^{*}=\frac{1}{r^{*}} \frac{\partial \psi^{*}}{\partial r^{*}}, \quad v^{*}=-\frac{1}{r^{*}} \frac{\partial \psi^{*}}{\partial x^{*}}
$$

the continuity equation (4.3) is recast as an elliptic Poisson equation

$$
\frac{\partial^{2} \psi^{*}}{\partial x^{* 2}}+\frac{\partial^{2} \psi^{*}}{\partial r^{* 2}}-\frac{1}{r^{*}} \frac{\partial \psi^{*}}{\partial r^{*}}+r^{*} \Omega^{*}=0
$$

The two equations, Equations (4.4) and (4.7), form a system of second-order nonlinear partial differential equations. The solution for the vorticity and stream function can only be obtained numerically subject to the appropriate boundary conditions. Because both of these equations are elliptic in nature, the numerical simulation requires boundary conditions on the entire boundary of a closed domain.

\subsubsection{Recovery of the Pressure Fields}

The velocity field may be calculated directly from the stream functions that are obtained during the application of the vorticity-stream function procedure. However, the pressure field must be recovered from the original momentum equations 
using these velocity results. The following second order, dimensionless pressure equation can be derived by appropriately differentiating and summing up the axial and radial momentum equations (4.1) and (4.2) (Roache, 1972):

$$
\frac{\partial^{2} p^{*}}{\partial x^{* 2}}+\frac{\partial^{2} p^{*}}{\partial r^{* 2}}+\frac{1}{r^{*}} \frac{\partial p^{*}}{\partial r^{*}}=S_{p}^{*}
$$

where the source term

$$
\begin{aligned}
S_{p}^{*} & =\Omega^{* 2}-u^{*}\left(\frac{\partial \Omega^{*}}{\partial r^{*}}+\frac{\Omega^{*}}{r^{*}}\right)+v^{*} \frac{\partial \Omega^{*}}{\partial x^{*}}- \\
& -\left[\frac{\partial^{2}}{\partial x^{* 2}}\left(\frac{u^{* 2}+v^{* 2}}{2}\right)+\frac{\partial^{2}}{\partial r^{* 2}}\left(\frac{u^{* 2}+v^{* 2}}{2}\right)+\frac{1}{r^{*}} \frac{\partial}{\partial r^{*}}\left(\frac{u^{* 2}+v^{* 2}}{2}\right)\right] .
\end{aligned}
$$

This is a Poisson form of the pressure equation. In the solution of Equation (4.8), the boundary conditions are obtained as normal gradients of pressure, determined from the primitive momentum equations (4.1) and (4.2) after the velocity has been determined. That is, the Neumann boundary condition, specifically $\frac{\partial p^{*}}{\partial \mathbf{n}}$, where $\mathbf{n}$ is the normal to the boundary, is imposed. As mentioned by Roache (1972), the numerical solution of this system does not converge. This conclusion was confirmed in the present study even though the scheme recommended by Miyakota (1962), Briley (1974) and Ghia et al. (1979) were utilized - the solution was found to drift slowly but endlessly.

An alternative to the above differential equation method is the integration of one of the momentum equations (Equations (4.1) and (4.2)) to obtain the pressure field (e.g., Payatakes et al., 1973; Azzam and Dullien, 1977). However, the accuracy of the integral method depends on the accuracy of the numerical integration scheme, and the path of integration (i.e., from left to right or from centerline to wall). It was found in the present study that the pressure distributions calculated by the integral method did not satisfy the axial and radial momentum equations simultaneously within a certain error tolerance. 
Based on the results described above, a hybrid of the differential equation method and the integral method has been developed (Ma and Ruth, 1991, 1993a). The details of this new scheme are presented in the next chapter.

\subsection{Implementation of Boundary Conditions}

Given the elliptic nature of the governing equations (Equations (4.4), (4.7) and (4.8)), boundary conditions are to be prescribed at all the boundaries of the computational domain. The correct implementation of the appropriate boundary conditions is crucial in obtaining meaningful and accurate results.

The boundary conditions for the flow problem under consideration can be divided into three parts, namely, the fluid/solid interface condition, the entrance and exit condition, and the symmetry condition.

\subsubsection{Fluid/Solid Interface Boundary Condition}

The stream function $\psi^{*}$ :

On the interface of fluid and solid (the line of $\mathrm{A}-\mathrm{B}-\mathrm{C}-\mathrm{D}-\mathrm{E}-\mathrm{F}$ ) in Figure 4.2 , viscous flow satisfies the condition of no-slip and zero suction and injection, thus both components of the velocity are set equal to zero, i.e. $u^{*}=v^{*}=0$. Accordingly, the stream function must remain constant along the interface:

$$
\psi^{*}=\text { const },
$$

and any constant value of $\psi^{*}$ may be selected. 


\section{The vorticity $\Omega^{*}$ :}

The evaluation of vorticity values along the interface $A-B-C-D-E-F$ is extremely important and is often the cause of trouble in getting a converged solution. In this research, the first order form of the wall vorticity (Roache, 1972) has been employed. This condition may easily be derived from the stream function equation (4.7). Applying that equation on the fluid/solid interface which is parallel to the $x$-axis, for example, by the no-slip condition

$$
\frac{1}{r^{*}} \frac{\partial \psi^{*}}{\partial r^{*}}=u^{*}=0
$$

and

$$
-\frac{1}{r^{*}} \frac{\partial^{2} \psi^{*}}{\partial x^{* 2}}=\frac{\partial}{\partial x^{*}}\left(-\frac{1}{r^{*}} \frac{\partial \psi^{*}}{\partial x^{*}}\right)=\frac{\partial v^{*}}{\partial x^{*}}=0
$$

which leads to

$$
\Omega_{w}^{*}=-\frac{1}{r^{*}} \frac{\partial^{2} \psi^{*}}{\partial r^{* 2}}
$$

Here the subscript $w$ refers to a value at the appropriate boundary point. For the fluid/solid interface parallel to the $r$-axis (Figure 4.2), a similar expression for the wall vorticity values may be written in the form

$$
\Omega_{w}^{*}=-\frac{1}{r^{*}} \frac{\partial^{2} \psi^{*}}{\partial x^{* 2}}
$$

The pressure:

According to the new approach for pressure computations given in Section 5.2, the required pressure boundary values on the fluid/solid interface are recovered by integrating Equations (4.1) and (4.2) at that boundary. For this purpose, Equations (4.1) and (4.2) are applied on the no-slip interface, and the working equations for the boundary pressure integrations can then be expressed in terms of the vorticity: 
Wall with normal in $r$-direction:

$$
\frac{\partial p^{*}}{\partial x^{*}}=-\frac{1}{R e_{d}}\left(\frac{\partial \Omega^{*}}{\partial r^{*}}-\frac{\Omega^{*}}{r^{*}}\right)
$$

Wall with normal in $x$-direction:

$$
\frac{\partial p^{*}}{\partial r^{*}}=\frac{1}{R e_{d}} \frac{\partial \Omega^{*}}{\partial x^{*}}
$$

Equations (4.13) and (4.14) are numerically integrated along the wall started from a given pressure level.

\subsubsection{Entrance and Exit Condition}

The boundary conditions at the entrance and exit of the solution domain are generally not known from the outset. Assuming that a steady state periodic fully developed solution exists (Payatakes et al., 1973; Patankar et al., 1977; Azzam and Dullein, 1977), it follows that the flow quantities (except pressure field) repeat themselves in a succession of cross sections that are separated from each other by the wavelength $L^{*}$. This statement may be expressed in the form

$$
\psi^{*}\left(x^{*}, r^{*}\right)=\psi^{*}\left(x^{*}+L^{*}, r^{*}\right)
$$

and

$$
\Omega\left(x^{*}, r^{*}\right)=\Omega^{*}\left(x^{*}+L^{*}, r^{*}\right)
$$

where $L^{*}$ is the dimensionless wavelength. Being a part of the solution, the values of stream function, $\psi^{*}$, and vorticity, $\Omega^{*}$, on the entrance and exit could be determined by an iterative procedure. The details of the numerical treatment of the conditions (4.15) and (4.16) are discussed in Chapter 6. 
The pressure values at the entrance and exit are determined by the integral method after the vorticity and stream function solutions are known. Through using the RUC proposed in this research, it has been found that the determination of the pressure values at the entrance and exit present less difficulty because both cross-sections have been taken as far as possible from the sudden geometry changes where steep changes of the flow variables are expected.

\subsubsection{The Symmetry Condition}

At the line of symmetry $\left(r^{*}=0\right)$, a zero normal gradient condition is assumed in the radial direction. This condition is equivalent to

$$
\begin{gathered}
v^{*}=0 \quad \text { at } r^{*}=0, \\
\psi^{*}=0 \quad \text { at } r^{*}=0, \\
\Omega^{*}=-\frac{\partial u^{*}}{\partial r^{*}}=0 \quad \text { at } r^{*}=0,
\end{gathered}
$$

and

$$
\frac{\partial p^{*}}{\partial r^{*}}=0 \quad \text { at } r^{*}=0
$$

\subsubsection{Condition At the Sharp Convex Corner}

Special consideration must be given to some corner points in the flow channel, because these corners are mathematical singular points for the vorticity and pressure fields and the conventional boundary conditions cannot be applied at these points. This will be a topic in Chapter 5 . 


\section{CHAPTER 5}

\section{NUMERICAL SCHEMES FOR}

\section{PORE FLOW COMPUTATIONS}

The mathematical formulation developed in Chapter 4 have laid the foundation for microscopic flow computations. To obtain reliable solutions of the problem, however, considerations have to be given to some outstanding problems in the application of this basic equation system. The treatment of the corner singularity and the pressure recovery in the vorticity-stream function approach, the two important features of the present numerical solutions, will form the subject of much of this chapter (Sections 5.1 and 5.2). The details of the computational procedure for microscopic flow fields will be discussed in Section 5.3.

\subsection{Treatment of the Corner Singularity}

The flow of an incompressible fluid around a re-entrant sharp corner is encountered in many engineering problems. For the RUC considered in this research, two re-entrant sharp corners are formed in the computational domain at the crosssections of the sudden expansion and contraction. These corners are mathematical singular points for the vorticity and pressure fields because of the no-slip boundary condition on the fluid/solid interface.

The theoretical situation very close to the corner has been described by Moffatt (1964) by making use of the argument that sufficiently near the corner the flow is 
Stokesian. By using diagonal grids at the corner, an extensive study of the laminar plane channel flow with an abrupt change in cross sectional area was performed by Dennis and Smith (1980). Their corner scheme may essentially be classified as an ad hoc method and is suitable for square grids only. Holstein and Paddon (1982) made a comparison of different ad hoc methods and their singular finite difference method which is based on the argument by Moffatt for the flow in contraction and expansion at low Reynolds numbers. In another study, Gupta et al. (1981) presented a power series solution in the vicinity of the corner and calculated the cavity flow for Reynolds numbers up to 400 .

In addition to these approaches, several ad hoc methods to circumvent the corner singularity have been devised (Kuwaguti, 1965; Woods, 1954; Greenspan, 1969; Thom and Apelt, 1961) and have been utilized by a number of following researchers (Vrentas and Duda, 1973; Webster, 1982; Nallasamy, 1986). A comprehensive examination of these methods for backstep flow in rectangular coordinates can be found in Roache and Mueller (1970).

In contrast, however, for high Reynolds number corner flow in a sudden contraction, which is characterized by local flow acceleration and separation, the validity of the above-mentioned methods is still not well understood. In this section, the various ad hoc methods will be briefly reviewed for the purpose of comparison. In view of the uncertainty of the ad hoc procedures and the locally small Reynolds number restriction of the Moffatt expansion method, a new scheme, the vorticity-circulation method, is developed (Ma and Ruth, 1993b).

\subsubsection{Traditional Corner Methods}

Equations (4.4) and (4.7) can be solved numerically subject to the set of boundary 
conditions $(4.10) \sim(4.20)$ by a finite difference procedure using rectangular grid lines parallel to the axes of $x^{*}$ and $r^{*}$. For a general quantity $\Phi^{*}$, the corresponding finite difference equations of (4.4) and (4.7) may be expressed in a successive substitution form

$$
\Phi_{i, j}^{*}=c_{1} \Phi_{i+1, j}^{*}+c_{2} \Phi_{i-1, j}^{*}+c_{3} \Phi_{i, j+1}^{*}+c_{4} \Phi_{i, j-1}^{*}+\text { source }
$$

where $c_{1}, c_{2}, c_{3}, c_{4}$ and source are the corresponding coefficients and source term in the iteration process, and $(i, j)$ are the grid indexes in the $\left(x^{*}, r^{*}\right)$ coordinate directions. On the fluid/solid interface, the stream function at the sharp corner presents no problem. The usual five-point difference equation (5.1) can be used at the neighboring grids of the corner $C$ shown in Figure 5.1. However, the velocity gradient has a jump discontinuity at that corner and the vorticity, by the definition (4.5), is singular there. The difference scheme (5.1) cannot be applied in the normal way at the neighbouring points of the corner and special treatment of this singularity is required. In the following, the four most often used ad hoc corner procedures as well as the Moffatt expansion method are briefly discussed.

\subsubsection{Ad hoc Methods}

In the usual grid structure, the corner $C$ is a grid point. The need for the corner vorticity $\Omega_{C}^{*}$ arises from the use of the central finite difference approximation for vorticity values at the neighboring points $W$ and $S$ (Figure 5.1). The ad hoc methods effectively impose extra conditions in different ways at the corner, with the aim of assigning a value of vorticity to the corner point. 


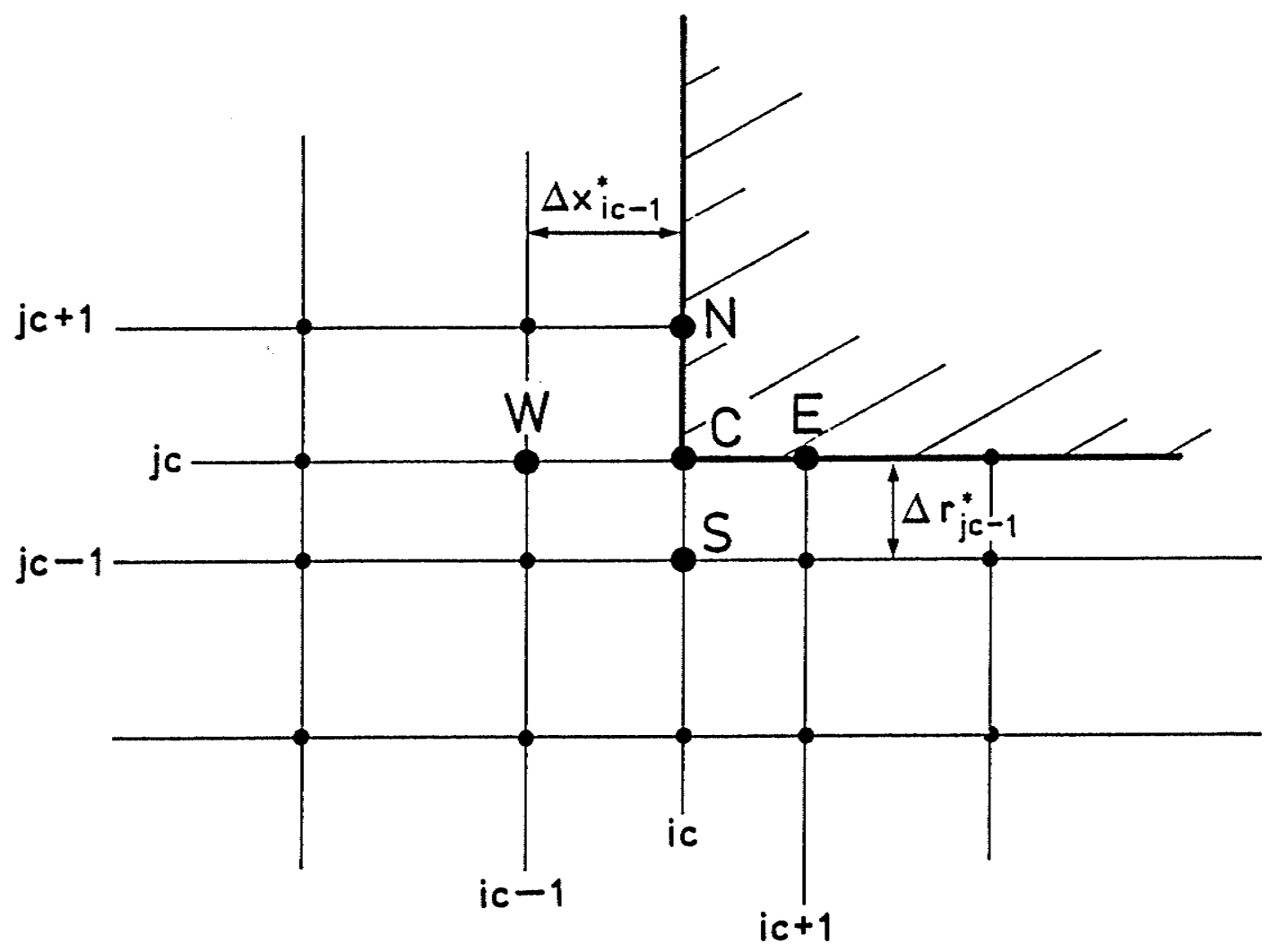

Fig. 5.1. The notation for corner vorticity computations. 
Discontinuous values $\quad$ Referring to Figure 5.1, two different vorticity values $\Omega_{U}^{*}$ and $\Omega_{D}^{*}$ are calculated respectively by

$$
\Omega_{U}^{*}=2 \frac{\left(\psi_{i c, j c}^{*}-\psi_{i c-1, j c}^{*}\right)}{r_{j c}^{*}\left(\Delta x_{i c-1}^{*}\right)^{2}}
$$

and

$$
\Omega_{D}^{*}=2 \frac{\left(\psi_{i c, j c}^{*}-\psi_{i c, j c-1}^{*}\right)}{r_{j c}^{*}\left(\Delta r_{j c-1}^{*}\right)^{2}}
$$

where $\Delta x_{i c-1}^{*}$ and $\Delta r_{j c-1}^{*}$ are the local step lengths in the $\left(x^{*}, r^{*}\right)$ coordinate directions. When the corner vorticity $\Omega_{C}^{*}$ is applied in a difference equation about node $W(i c-1, j c)$ just upstream of the contraction, $\Omega_{C}^{*}=\Omega_{U}^{*}$ is used; however, $\Omega_{C}^{*}=\Omega_{D}^{*}$ is employed for the node $S(i c, j c-1)$.

Average of wall values A single corner vorticity equal to the average of the two wall values is imposed in this method

$$
\Omega_{C}^{*}=\frac{\Omega_{U}^{*}+\Omega_{D}^{*}}{2}=\frac{\psi_{i c, j c}^{*}-\psi_{i c-1, j c}^{*}}{r_{j c}^{*}\left(\Delta x_{i c-1}^{*}\right)^{2}}+\frac{\psi_{i c, j c}^{*}-\psi_{i c, j c-1}^{*}}{r_{j c}^{*}\left(\Delta r_{j c-1}^{*}\right)^{2}} .
$$

Zero corner vorticity This method is an attempt to force separation to occur at the corner by imposing

$$
\Omega_{C}^{*}=0
$$

Although all the previous evidence suggests that separation occurs just after the sharp corner, as an ad hoc method referred to frequently, this scheme has also been tested in the present research.

$\psi^{*}$-symmetry about corner point Assuming the stream function is symmetric about the corner point, that is, $\psi_{i c-1, j c}^{*}=\psi_{i c+1, j c}^{*}$ and $\psi_{i c, j c-1}^{*}=\psi_{i c, j c+1}^{*}$, the corner vorticity is then evaluated from

$$
\Omega_{C}^{*}=\frac{2}{r_{j c}^{*}}\left[\frac{\psi_{i c, j c}^{*}-\psi_{i c-1, j c}^{*}}{\left(\Delta x_{i c-1}^{*}\right)^{2}}+\frac{\psi_{i c, j c}^{*}-\psi_{i c, j c-1}^{*}}{\left(\Delta r_{j c-1}^{*}\right)^{2}}\right] .
$$




\subsubsection{Moffatt Expansion Method}

Assuming that the local Reynolds number is very small, near the corner $C$ the stream function $\psi^{*}$ satisfies the Stokes equation

$$
\nabla^{4} \psi^{*}=0
$$

An expansion of $\psi^{*}$ about the sharp corner $C$ may be obtained by applying the general solution (derived by Moffatt, 1964) to the present geometry and flow situation:

$$
\begin{aligned}
\psi^{*}=\psi_{0}^{*}+A_{1} r_{C}^{* \lambda_{1}} & \left\{\cos \left[\left(\lambda_{1}-2\right) \frac{3}{4} \pi\right] \cos \left(\lambda_{1} \theta^{*}\right)-\right. \\
& \left.-\cos \left(\lambda_{1} \frac{3}{4} \pi\right) \cos \left[\left(\lambda_{1}-2\right) \theta^{*}\right]\right\}+ \\
+B_{1} r_{C}^{* \lambda_{2}} & \left\{\sin \left[\left(\lambda_{2}-2\right) \frac{3}{4} \pi\right] \sin \left(\lambda_{2} \theta^{*}\right)-\right. \\
& \left.-\sin \left(\lambda_{2} \frac{3}{4} \pi\right) \sin \left[\left(\lambda_{2}-2\right) \theta^{*}\right]\right\},
\end{aligned}
$$

where $\left(r_{C}^{*}, \theta^{*}\right)$ are the local dimensionless polar coordinates originating at point $C$ and $\lambda_{1}$ and $\lambda_{2}$ are the dominant eigenvalues of the relevant antisymmetrical and symmetrical problems. $A_{1}$ and $B_{1}$ are the constants depending on the particular flow; $\psi_{0}^{*}$ is the stream function value on the wall boundary.

To evaluate the vorticity values for the neighboring points ( $W$ and $S$ ) of the sharp corner, the vorticity expansion at the point $C$ may be obtained by using Equation (5.8) and the polar form of Equation (4.7):

$$
\begin{aligned}
\Omega^{*}=-4\{ & A_{1}\left(\lambda_{1}-1\right) r_{C}^{* \lambda_{1}-2} \cos \left(\lambda_{1} \frac{3}{4} \pi\right) \cos \left[\left(\lambda_{1}-2\right) \theta^{*}\right]+ \\
& \left.+B_{1}\left(\lambda_{2}-1\right) r_{C}^{* \lambda_{2}-2} \sin \left(\lambda_{2} \frac{3}{4} \pi\right) \sin \left[\left(\lambda_{2}-2\right) \theta^{*}\right]\right\}
\end{aligned}
$$

Two constants $A_{1}$ and $B_{1}$ in Equation (5.9) may be determined in the computations by the vorticity values at the points $N(i c, j c+1)$ and $E(i c+1, j c)$ in Figure 5.1. Then Equation $(5.1)$ can be used to calculate the vorticity values at points $W$ and 
$S$, where the normal finite difference method cannot be applied because of the singularity of the vorticity at the point $C$.

The success of the Moffatt expansion is dependent entirely on the validity of the local Stokesian flow assumption. It is not surprising that this method becomes less efficient in the present problem because of the facts that for the flow around re-entrant corners, especially the corner at the contraction, the magnitude of the velocity increases greatly in the small pipe according to the area ratio and the velocity profiles are quite flat at the corner region (see Subsection 5.1.3).

\subsubsection{The Vorticity-Circulation Method}

It is known that the vorticity of the flow field is closely related to the circulation of the flow. The new scheme uses the circulation concept. Instead of creating a fictitious corner vorticity as all the ad hoc methods do, an open surface $A^{*}$ which is enclosed by contour $L_{L}^{*}$ is constructed (Figure 5.2). The corner point is not included in $A^{*}$. Stokes theorem for the region $A^{*}$ may then be written as

$$
\Gamma^{*}=\oint_{L_{L}^{*}}\left(u^{*} d x^{*}+v^{*} d r^{*}\right)=\iint_{A^{*}} \Omega^{*} d A^{*},
$$

where $\Gamma^{*}$ is the circulation defined as the line integral of the velocity and $d A^{*}$ is the area element. The circulation about the closed curve $L_{L}^{*}$ is equal to the vorticity integral over the surface $A^{*}$ bounded by $L_{L}^{*}$.

Assuming that an area $A^{*}$ has been chosen (as shown in Figure 5.2), the circulation $\Gamma^{*}$ along the contour $L_{L}^{*}$ can be calculated using the velocity fields of the $(m-1) t h$ iteration (the values of the $m t h$ iteration are used where available). The vorticity flux on the right of Equation (5.10) except the contribution from the point $W$ (or $S$ ) can also be determined in the same way. A new vorticity value 


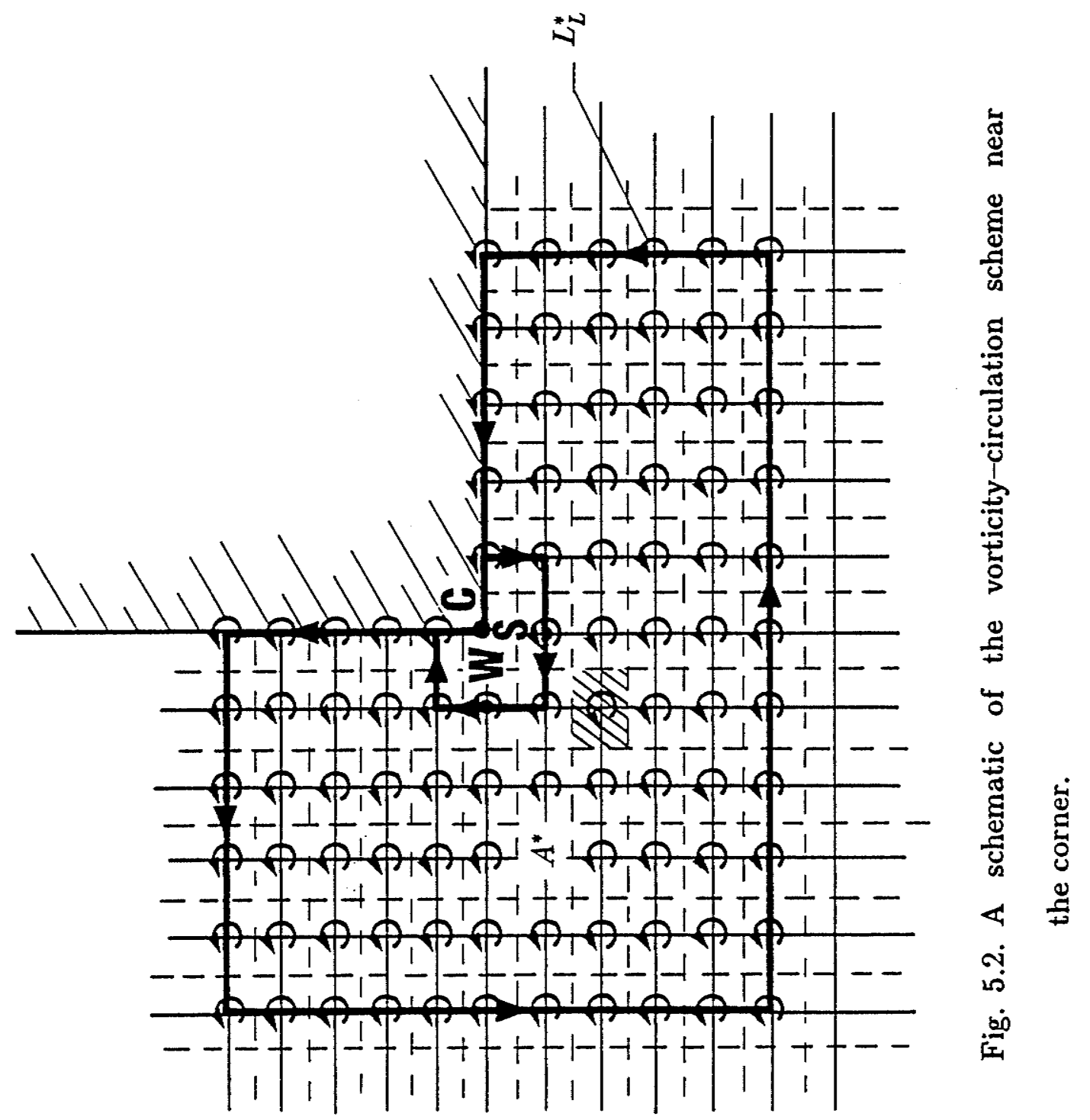


at the point $W$ (or $S$ ) then may be calculated by Equation $(5.10)$ using the $(m-1)$ th (or $m t h$ ) vorticity value at $S$ (or $W$ ) as an approximation. In the process of numerical integration, the following assumptions have been made:

a. the effect of the geometrically sharp corner is rounded off by viscous effects, so that the vorticity is continuous everywhere over the surface $A^{*}$;

b. for simplicity, the vorticity is assumed to be uniform in each unit block bounded by dashed lines and shown by the hatched area in Figure 5.2;

c. the velocity is linearly distributed between the computational grid points.

By employing Equation (5.10) and the assumptions above, the vorticity value at the point $W$ (similarly at $S$ ) in a nomuniform grid system can be expressed as

$$
\begin{aligned}
\Omega_{W}^{*}=\frac{4}{\Delta x_{i c-2}^{*}\left(\Delta r_{j c-1}^{*}+\Delta r_{j c}^{*}\right)} & {\left[\oint_{L_{L}^{*}} u^{*} d x^{*}+v^{*} d r^{*}-\iint_{A_{i}^{*}} \Omega^{*} d A^{*}\right]-} \\
& -\frac{\Delta r_{j c-2}^{*}\left(\Delta x_{i c-1}^{*}+\Delta x_{i c}^{*}\right)}{\Delta x_{i c-2}^{*}\left(\Delta r_{j c-1}^{*}+\Delta r_{j c}^{*}\right)} \Omega_{S}^{*},
\end{aligned}
$$

where $A_{i}^{*}$ denotes the area excluding the unit blocks at $W$ (or $S$ ) in $A^{*}$. The vorticity values at the grids $W$ and $S$ have to be calculated iteratively in association with the numerical method for the field computations (see Section 5.3 for details), until satisfactory convergence is achieved. A comparison of this scheme with the other methods will be given in Subsection 5.1.3.

\subsubsection{Validity of the Vorticity-Circulation Method}

The axial velocity distributions in the neighborhood of the sharp corner as calculated by the vorticity-circulation method are first compared with the ad hoc treatments and the experimental results of Durst and Loy (1985) for a sudden contraction flow at a series of Reynolds numbers. 
Two typical cases $\left(R e_{d}=43\right.$ (Figure 5.3) and $R e_{d}=1813$ (Figure 5.4)) indicate that the choice of the ad hoc methods affects considerably the computed profiles in the region of the corner (especially at high Reynolds numbers), and the validity of these methods changes at different Reynolds numbers. It may also be seen that as a unified corner treatment, the vorticity-circulation method predicts good results at both high and low Reynolds numbers.

Figure 5.5 shows a comparison of the axial velocity profiles of the vorticitycirculation method with the experimental data of Durst and Loy (1985) for $R e_{d}=698$. The results of the finite volume SIMPLE method reported by Peric et al. (1988) are also included at eight different length stations, four before, and four after the contraction. Generally, the results obtained by the vorticitycirculation method agreed well with both the experimental and SIMPLE results.

\subsection{Pressure Recovery: The Hybrid Method}

In order to extract pressure fields from the numerical solutions of the vorticity and stream function, the differential method and the integral method have generally been used (Roache, 1972) and both methods have difficulties in determining accurate pressure fields for the geometry considered in this study. In this section, following a brief review of the conventional methods, a new approach for the pressure recovery is presented.

\subsubsection{The Conventional Methods}

To recover the pressure fields from the known vorticity-stream function solutions, the following two methods have been widely used. 


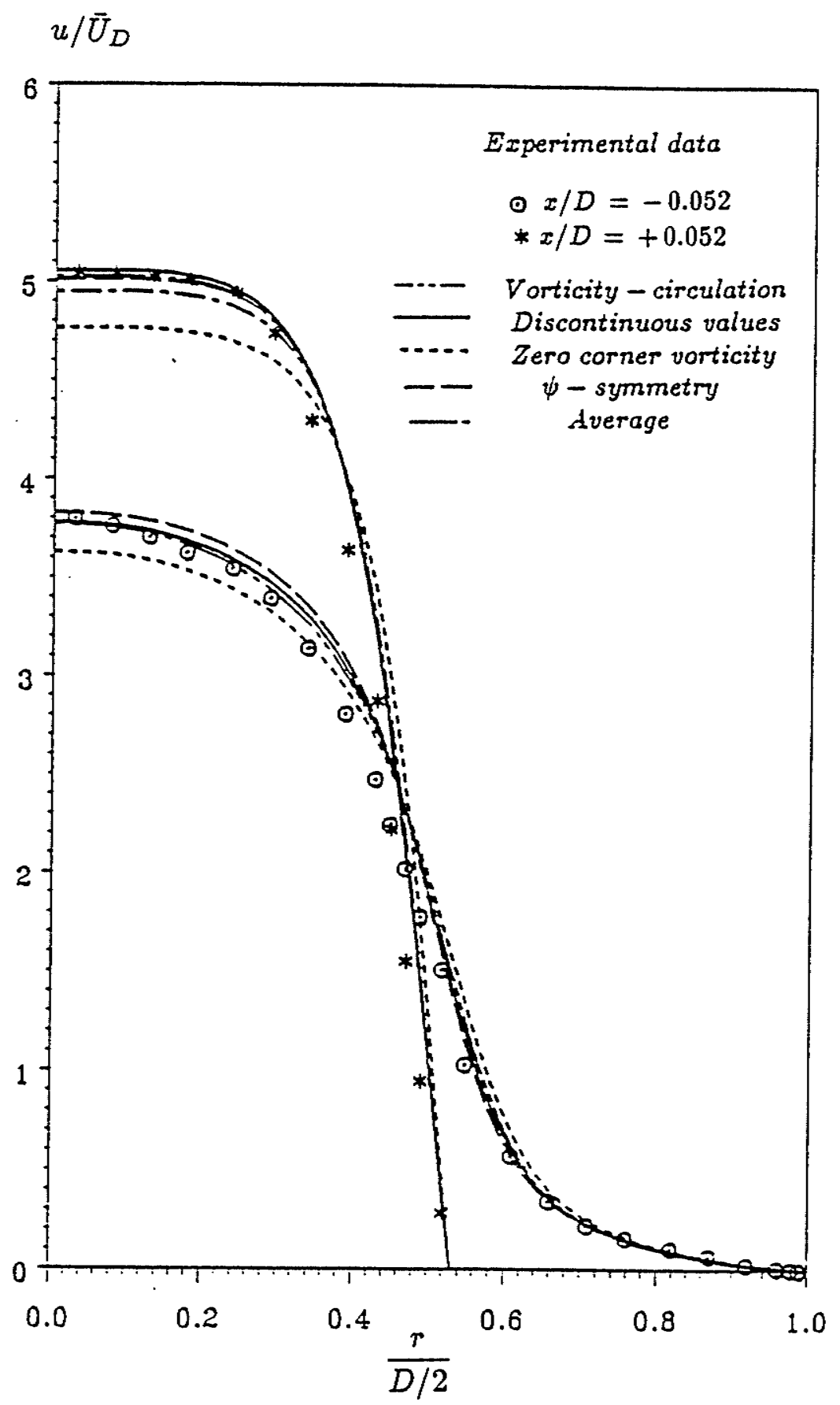

Fig. 5.3. A comparison of the axial velocity profiles, $R e_{d}=43$. 


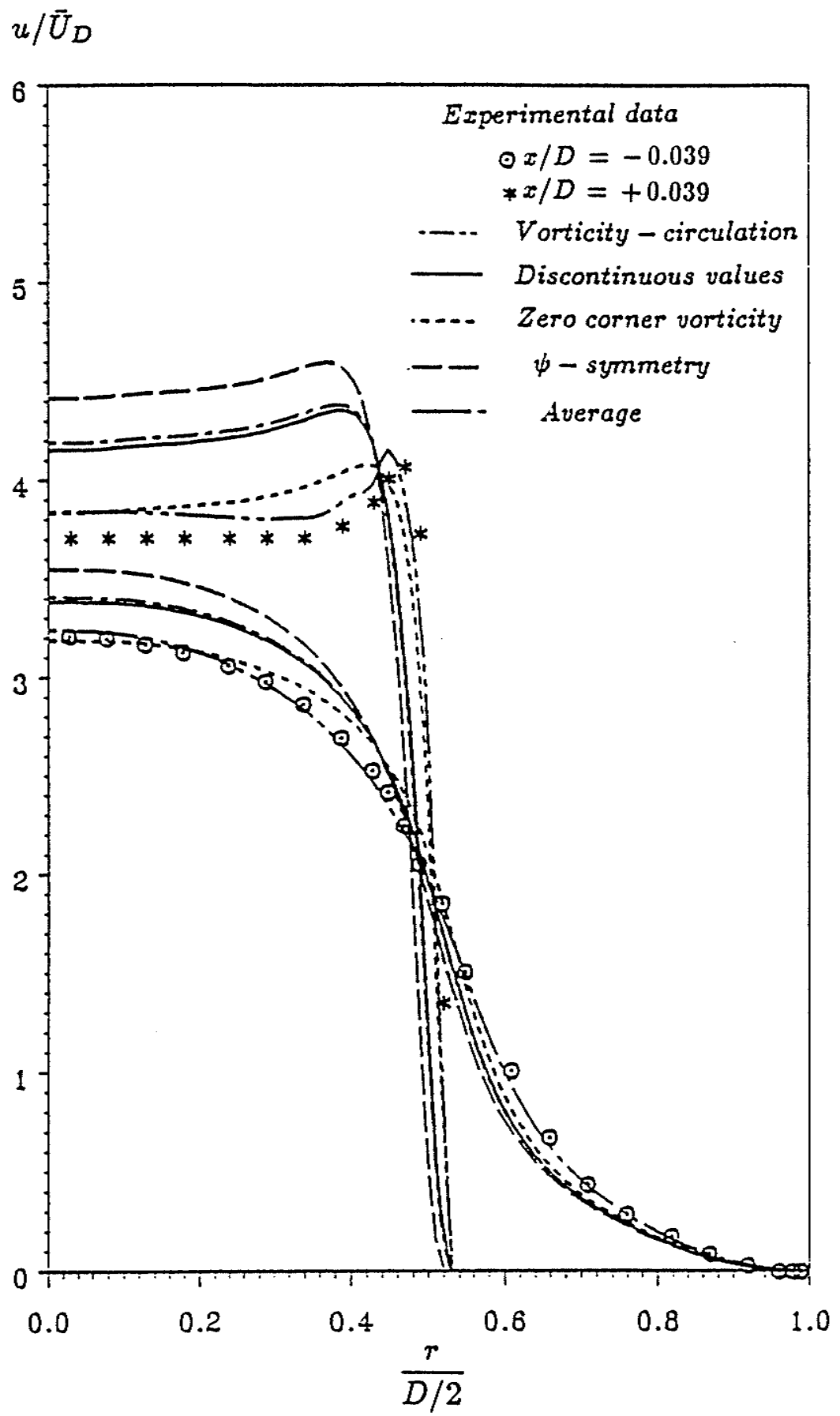

Fig. 5.4. A comparison of the axial velocity profiles, $R e_{d}=1813$. 

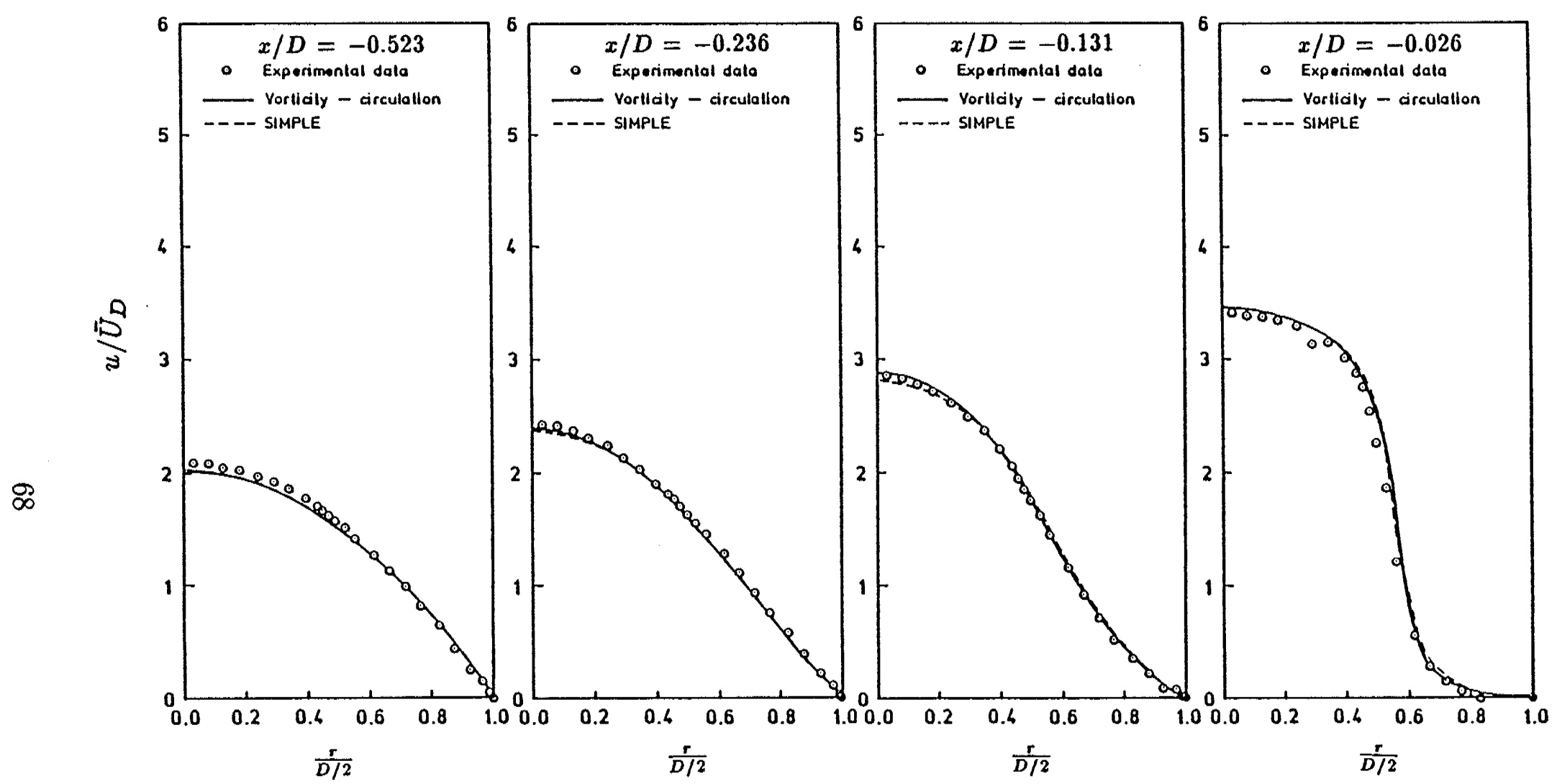

(A). Before the contraction

Fig. 5.5. Comparison of computed and measured profiles of axial velocity for $R e_{d}=698$. 


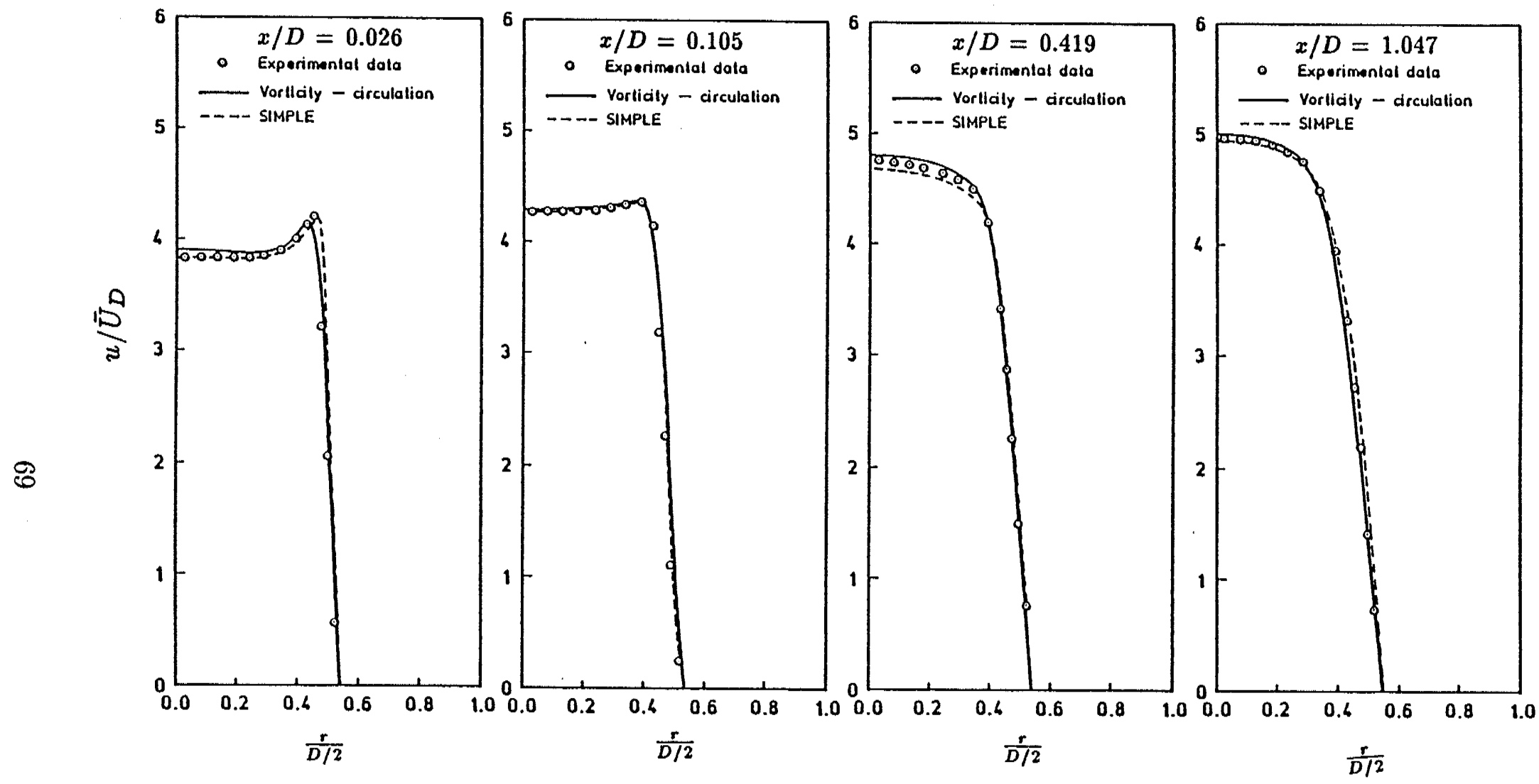

(B). After the contraction

Fig. 5.5. Comparison of computed and measured profiles of axial velocity for $R e_{d}=698$. 
The integral method One of the momentum equations (4.1) and (4.2) may be numerically integrated by starting from an arbitrary point with an arbitrary integration constant (Macagno and Hung, 1967; Vrentas and Duda, 1973; Nallasamy, 1986). This evaluation process involves both differentiation and integration at each point in the computational domain. Depending on the computational grids and the path of integration, this method predicts acceptable results in the region not very close to the corners. However, for the interior points when the path of the integration is close to the sharp corners, it was found that the pressure distributions given by the integral method did not satisfy the $x^{*}$ - and $r^{*}$ - momentum equations simultaneously.

The differential method A system which is composed of the Poisson pressure equation (4.8) and the Neumann boundary condition may be solved iteratively. As already mentioned in Subsection 4.2 .2 , the iterative process to this equation does not converge.

\subsubsection{The Hybrid Method}

To obtain accurate pressure solutions, a hybrid of the differential method and the integral method was utilized in this study. Specifically, the integral method was used to evaluate the wall pressure distribution, where the integrals are greatly simplified because of the no-slip condition, then the pressure (Poisson) equation was solved iteratively with the now known boundary values. In essence, the Poisson problem with Neumann boundary conditions was converted to a Poisson problem with Dirichlet boundary conditions.

By applying the simple trapezoidal rule (Press et al., 1987), the wall pressure integration may be derived from Equation (4.13) and written in the successive 
substitution form:

$$
p_{i}^{*}=p_{i+1}^{*}+\frac{\Delta x_{i}^{*}}{2} \frac{1}{R e_{d}}\left[\left(\frac{\partial \Omega^{*}}{\partial r^{*}}+\frac{\Omega^{*}}{r^{*}}\right)_{i}+\left(\frac{\partial \Omega^{*}}{\partial r^{*}}+\frac{\Omega^{*}}{r^{*}}\right)_{i+1}\right] \text {, }
$$

where $i$ is the grid index in the $x^{*}$ - direction and $\Delta x_{i}^{*}$ is the local grid length. The quantities in the angle bracket on the right may be determined numerically from the vorticity solutions.

The failure of the two conventional methods arises mainly from the large computational error in the vicinity of the sharp convex corners where dramatic changes of the flow quantities occur. To limit the effects of this error in the wall pressure determination, a special integration path which avoids the involvement of the sharp corners in the computation has been utilized.

With all the boundary values being assigned, the Poisson pressure equation (4.8) may be solved numerically by an iterative procedure introduced in the next section. A pressure comparison between the hybrid method and the experimental data will be given in Chapter 7 .

\subsection{Computational Procedure for Flow}

\section{Equations}

The governing equations (4.4), (4.7) and (4.8) presented in Chapter 4 are a system of non-linear partial differential equations. The system cannot be solved analytically and, therefore, must be solved by numerical methods. In this analysis, a numerical method which is similar in certain aspects to the one developed by Chien (1977a, b) was utilized, with the important differences being the treatment of the vorticity boundary condition and the recovery method of the pressure fields. In this section, a standard form of the governing equations is first derived to represent 
the common feature of these equations. A general finite difference formulation is then developed so that stable and convergent solutions can be obtained for a wide range of flow conditions.

\subsubsection{The Finite Difference Formulation}

Numerical solutions of Equations (4.4), (4.7), and (4.8) were obtained using the finite difference approximations. To simplify the analysis, these equations can be cast in a standard form which retains the basic features such as the diffusion, the convection, the production and the dissipation of a flow variable. The standard form derived is

$$
\left(a_{1} \frac{\partial^{2} \Phi^{*}}{\partial x^{* 2}}+a_{2} \frac{\partial^{2} \Phi^{*}}{\partial r^{* 2}}\right)-\left(b_{1} \frac{\partial \Phi^{*}}{\partial x^{*}}+b_{2} \frac{\partial \Phi^{*}}{\partial r^{*}}\right)=s,
$$

where $\Phi^{*}$ represents the flow variables, i.e., $\Omega^{*}, \psi^{*}$ and $p^{*}, a_{1}, a_{2}, b_{1}, b_{2}$ and $s$ are the corresponding coefficients and source term for each of the variables.

The finite difference formulation of Equation (5.13) can be achieved by applying the conventional second-order central difference scheme for each terms. In a nonuniform grid system, it takes the following form

$$
\begin{aligned}
& \frac{a_{1}}{G_{i}}\left\{2\left[\frac{1}{\left(\Delta x_{i, j}^{*}+\Delta x_{i+1, j}^{*}\right)}\left(\frac{\Phi_{i+1, j}^{*}}{\Delta x_{i+1, j}^{*}}+\frac{\Phi_{i-1, j}^{*}}{\Delta x_{i, j}^{*}}\right)-\frac{\Phi_{i, j}^{*}}{\Delta x_{i, j}^{*} \Delta x_{i+1, j}^{*}}\right]-\left(\frac{b_{1}}{a_{1}} G_{i}\right)\right. \\
& \left.\times\left[\frac{1}{\Delta x_{i, j}^{*}+\Delta x_{i+1, j}^{*}}\left(\frac{\Delta x_{i, j}^{*} \Phi_{i+1, j}^{*}}{\Delta x_{i+1, j}^{*}}-\frac{\Delta x_{i+1, j}^{*} \Phi_{i-1, j}^{*}}{\Delta x_{i, j}^{*}}\right)+\frac{\left(\Delta x_{i+1, j}^{*}-\Delta x_{i, j}^{*}\right) \Phi_{i, j}^{*}}{\Delta x_{i, j}^{*} \Delta x_{i+1, j}^{*}}\right]\right\} \\
& +\frac{a_{2}}{G_{j}}\left\{2\left[\frac{1}{\left(\Delta r_{i, j}^{*}+\Delta r_{i, j+1}^{*}\right)}\left(\frac{\Phi_{i, j+1}^{*}}{\Delta r_{i, j+1}^{*}}+\frac{\Phi_{i, j-1}^{*}}{\Delta r_{i, j}^{*}}\right)-\frac{\Phi_{i, j}^{*}}{\Delta r_{i, j}^{*} \Delta r_{i, j+1}^{*}}\right]-\left(\frac{b_{2}}{a_{2}} G_{j}\right)\right. \\
& \left.\left.\times\left[\frac{\Delta r_{i, j}^{*} \Phi_{i, j+1}^{*}}{\Delta r_{i, j+1}^{*}}+\frac{\Delta r_{i, j+1}^{*} \Phi_{i, j-1}^{*}}{\Delta r_{i, j}^{*}}\right)+\frac{\left(\Delta r_{i, j+1}^{*}-\Delta r_{i, j}^{*}\right) \Phi_{i, j}^{*}}{\Delta r_{i, j}^{*} \Delta r_{i, j+1}^{*}}\right]\right\} \\
& \quad=0,
\end{aligned}
$$


where the decay function $G_{i}$ and $G_{j}$, according to Chien (1977b), are evaluated locally as

$$
G= \begin{cases}1.0-0.0625 R^{2}, & \text { for }|R| \leq 2 \\ \frac{2}{|R|}-\frac{1}{R^{2}}, & \text { for }|R| 2\end{cases}
$$

where $G=G_{i}$, when $R=\frac{b_{1}}{a_{1}} \Delta x_{i, j}^{*}$ and $G=G_{j}$, when $R=\frac{b_{2}}{a_{2}} \Delta r_{i, j}^{*}$.

The stability limitation associated with the central difference scheme and accuracy problem inherent to the up-wind difference scheme are avoided by the use of the locally evaluated decay functions in the finite difference formulation. In fact, the introduction of the decay function will provide a smooth transition from the conventional central-difference scheme to the upwind-difference scheme (Chien, 1977b).

\subsubsection{Procedures of the Iteration Process}

The general difference equation (5.14) can be expressed in a successive substitution form

$$
\left(\Phi_{i, j}^{*}\right)^{(m+1)}=\frac{c_{1}\left(\Phi_{i+1, j}^{*}\right)^{(m)}+c_{2}\left(\Phi_{i-1, j}^{*}\right)^{(m+1)}+c_{3}\left(\Phi_{i, j+1}^{*}\right)^{(m)}+c_{4}\left(\Phi_{i, j-1}^{*}\right)^{(m+1)}+s_{i, j}^{(m)}}{c_{u}} .
$$

where the superscript $m$ denotes the values at the $m t h$ iteration, $(m+1)$ denotes the updated value, the coefficients $c_{1}, c_{2}, c_{3}, c_{4}, c_{u}$ and the source term $s_{i, j}$ are given in APPENDIX D for each of the variables, $\Omega^{*}, \psi^{*}$ and $p^{*}$.

For simplicity, the Gauss-Seidel point iteration method has been used to perform the vorticity and the stream function calculations. The SOR (successive over-relaxation) method has been utilized in the iteration solutions of the pressure fields to accelerate the convergency. 


\subsubsection{Outline of the Solution Procedure}

As noted previously, an iterative procedure is followed to first generate the values of the vorticity and the stream function at each grid node satisfying the system of difference equations resulting from the descretization of Equations (4.4) and (4.7). Then the pressure field is recovered by solving a Poisson problem with the Dirichlet boundary condition. A general outline of the numerical solution procedure is given in Figure 5.6 and can be explained as follows:

1. The iteration procedure starts with a guess or estimate for the values of $\Omega^{*}$ and $\psi^{*}$ at all grid points. The initially guessed values of $\left(\Omega_{i, j}^{*}\right)^{(0)}$ and $\left(\psi_{i, j}^{*}\right)^{(0)}$ have been set to equal those of the fully developed Hagen-Poiseuille flow in the computations.

2. The computations of the vorticity and stream function form a main loop of iterations. The stream function is updated by the finite difference form of Equation (4.7) and the corresponding boundary conditions (4.10), (4.15) and (4.18). The vorticity distribution is determined from the substitution formula obtained as the discrete analogue to Equation (5.16). The updated $\psi^{*}$ values are used in the computations of $\Omega^{*}$. The vorticity values at the neighboring points of the convex corners are calculated according to the procedure given in Subsection 5.1.2. This iteration process is continued until satisfactory convergence achieved.

3. From the converged vorticity and stream function values, the pressure calculation is performed in a separate loop. The hybrid method introduced in Subsection 5.2 .2 is utilized in the present pressure computations. 


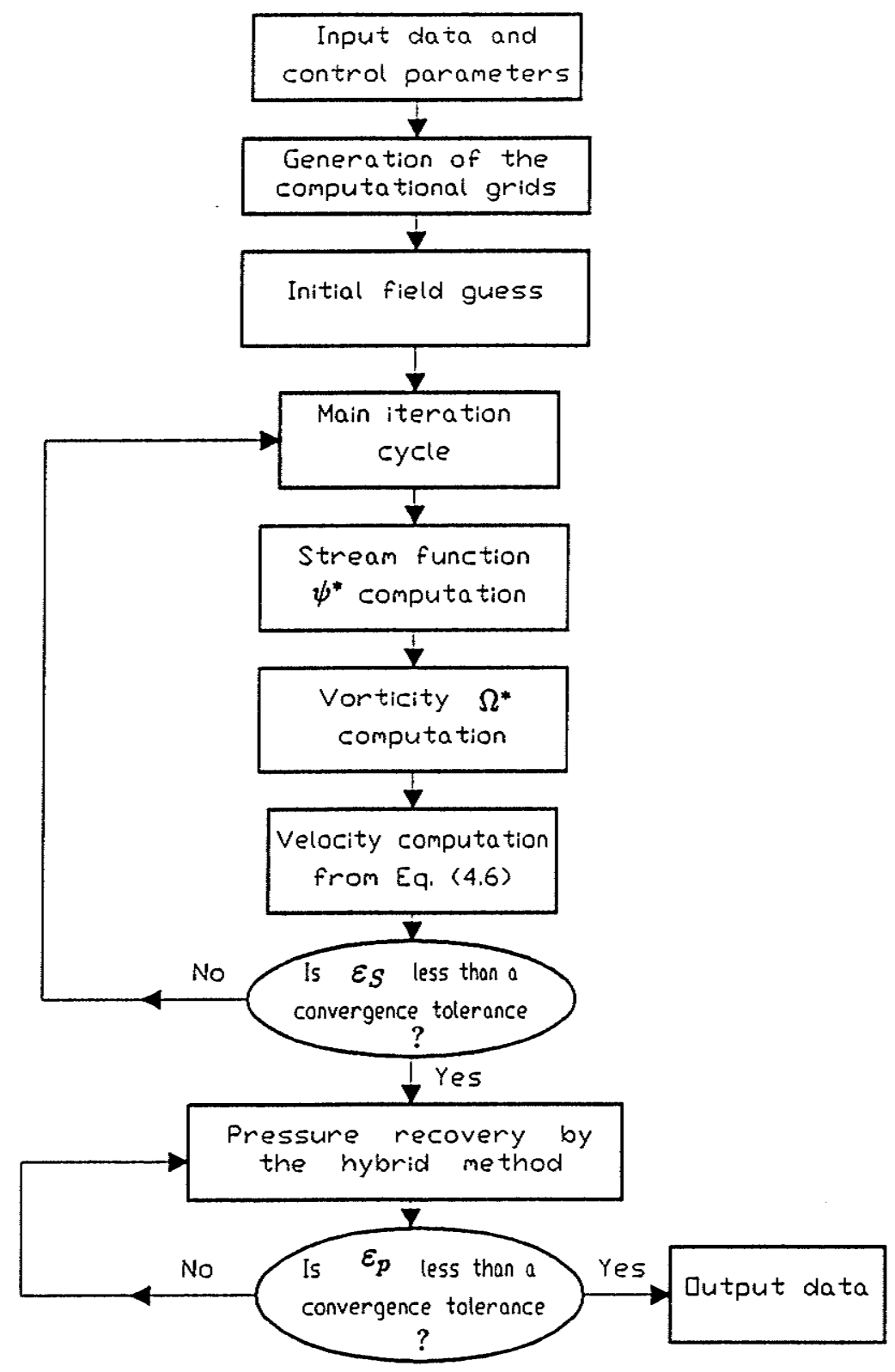

Fig. 5.6. Flow chart of the numerical solution procedure. 


\section{CHAPTER 6}

\section{COMPUTATIONAL DETAILS}

The development of a reliable numerical algorithm for microscopic level computations in high $\mathrm{F}$-number flow is crucial for the present analysis. Therefore, further consideration will be given to the characteristics of the accuracy, convergence and stability of the method in this chapter. More details about the discrete solution procedure will be presented. The discussion will start with the generation of the non-uniform grid on which the differential equations are discretized (Section 6.1). Later, the accuracy and the convergence of the solutions are examined in Section 6.2 and 6.3 , respectively. The last section (Section 6.4) will summarize the computer program developed in this research.

\subsection{Computational Grids}

To solve the partial differential equations by using the finite-difference procedure, the first step to be taken is to replace the continuous problem domain with a finitedifference mesh containing a finite number of grid points. The finite-difference grids utilized in this study are non-uniform in both axial and radial coordinate directions. A large number of grid point are placed in the corner region and near the fluid/solid interface where steep variations in computed quantities are expected. This allows accurate calculations to be performed without overly increasing the total number of computational points. In the present approach, simple independent variable transformations (Anderson et al., 1984) are used to generate the grids. 


\section{Transformation 1:}

A suitable transformation for a two-dimensional boundary layer type problem is given by

$$
y=h \nu \frac{\left(\frac{\nu+1}{\nu-1}\right)^{\bar{y}}-\nu}{1+\left(\frac{\nu+1}{\nu-1}\right)^{\bar{y}}}
$$

where $y$ represents a stretched coordinate and $\bar{y}$ represents the coordinate of grid points in a uniform system; $\nu$ is the stretching parameter. This stretching transformation clusters more grid points near $y=h$ if $\nu$ is chosen properly.

\section{Transformation 2:}

Another transformation needed is to refine the mesh about some interior point $y_{c}$. The following expression serves the purpose:

$$
y=y_{c}\left\{1+\frac{\sinh [\tau(\bar{y}-E)]}{\sinh (\tau E)}\right\}
$$

where

$$
E=\frac{1}{2 \tau} \ln \left[\frac{1+\left(e^{\tau}-1\right)\left(\frac{y_{c}}{h}\right)}{1+\left(e^{-\tau}-1\right)\left(\frac{y_{c}}{h}\right)}\right] \quad 0<\tau<\infty .
$$

In this transformation, $\tau$ is the stretching parameter which varies from zero (no stretching) to large values which produce the most refinement near $y=y_{c}$.

For flow computations of the diverging-converging representative unit cell considered in this research, the two transformations explained above have been used together. A sample of the resultant grid is shown in Figure 6.1.

\subsection{Accuracy of the Finite-Difference Solutions}

The finite-difference discretizations of the set of nonlinear coupled partial differ- 


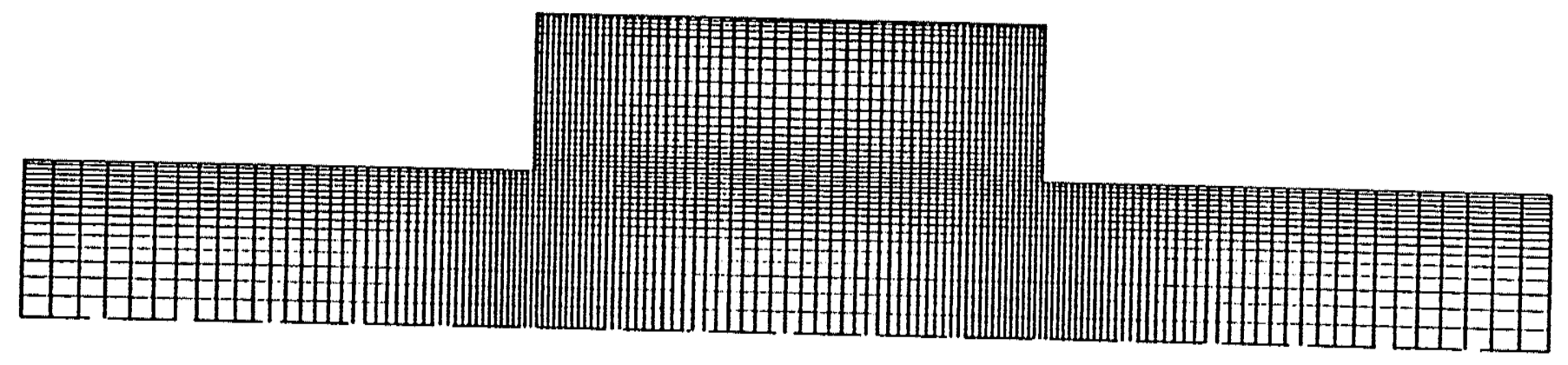

Fig. 6.1. Nonuniform computational grids for an RUC. 
ential equations (4.4), (4.7) and (4.8) lead to a system of simultaneous algebraic equation that has to be solved in an iterative fashion. To obtain a reliable numerical solution, it is essential that accuracy and consistency be maintained at every step of the computational procedure. In other words, the features of the discretization error and the round-off error of the numerical solutions should be fully taken into account in the development of the numerical method.

The discretization error results from replacement of the continuous problem by the discrete model and depends on the finite difference scheme and the grid size used in the computations. In the present work, all the derivatives have been represented by second-order accurate, central-difference formulas. In situations where the central-difference formulas cannot be used, second-order accurate onesided formulas are employed. For the solutions of Poisson type equations (4.4), (4.7) and (4.8), all the coefficient matrices used are diagonally dominant. So both Gauss-Seidel and SOR iterative procedures have led to stable, second-order accurate solutions. To control the level of the round-off error, on the other hand, all computations are performed using double precision arithmetic.

The choice of the grids is dependent on the accuracy desired and the computational storage and time available. In this study, the optimum grid size was obtained by carrying out multi-step numerical experiments because there were two relatively independent computational schemes, the vorticity-stream function and the pressure, involved.

\subsubsection{Accuracy of the Vorticity-Circulation Method}

To deal with the corner singularity, a new scheme, the vorticity-circulation method, has been introduced in Section 5.2. In the numerical integration of Equation (5.11), 
a linear velocity distribution between the grids and a uniform vorticity over the unit block have been assumed. This means that the trapezoidal rule is applied in both integrals and theoretically the error should reduce as the grid size decreases (Press et al., 1987). In an attempt to improve the integral accuracy, the higher-order Simpson's rule has also been tried. The numerical results showed that completely insignificant variations in the integral values occurred for the grids used in the calculations. To reconfirm the reliability of these assumptions, a conservation test was performed for the field quantities of a sudden contraction flow in the small pipe where the most severe change of the flow quantities exists. The numerical integrals of both sides of Equation (5.10), the circulation $\left(\Gamma^{*}\right)$ and the vorticity flux, were calculated for an area which is contoured by the solid boundary, the centerline, the inlet of the small pipe and a cross section close to the outlet. The results of the two cases $\left(R e_{d}=43\right.$ and 1813) are given in Table 6.1 and Table 6.2, respectively. The data in Table 6.1 show that the difference of the two integral values is well under $1 \%$ at $R e_{d}=43$ for all the grids used. The error increases at higher Reynolds numbers (Table 6.2). For the finest grids, however, this error is not larger than $4 \%$ for all the Reynolds numbers studied.

\begin{tabular}{|c|c|c|c|c|}
\hline \multicolumn{5}{|c|}{ Table 6.1 Conservation test of the vorticity-circulation method for $R e_{d}=43$} \\
\hline$R e_{d}=43$ & $137 \times 40$ & $163 \times 48$ & $189 \times 55$ & $208 \times 61$ \\
\hline Circu. $\left(\Gamma^{*}\right)$ & 9.7280 & 9.5423 & 9.6168 & 9.5260 \\
\hline Vort. Flux & 9.6285 & 9.5910 & 9.6633 & 9.5713 \\
\hline
\end{tabular}

Table 6.2 Conservation test of the vorticity-circulation method for $R e_{d}=1813$

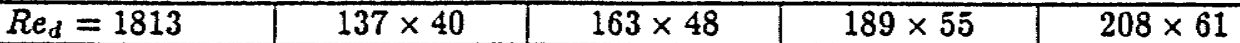

\begin{tabular}{|c|c|c|c|c|} 
Circu. $\left(\Gamma^{*}\right)$ & 6.9533 & 7.2405 & 7.4068 & 7.5588 \\
\hline
\end{tabular}

\begin{tabular}{|l|l|l|l|l|}
\hline Vort. Flux & 7.3086 & 7.5422 & 7.7236 & 7.8233 \\
\hline
\end{tabular}




\subsubsection{Accuracy Test for Pressure Computations}

The basic governing equations (4.1) and (4.2) represent a conservation of momentum, and the terms in these equations (i.e., the inertial, viscous and pressure), should be in balance at every point of the flow field. In order to check on the reliability of the pressure computation method, the three terms in these equations were determined for various flow conditions by substituting the velocity and pressure values obtained separately from vorticity-stream function and pressure computation loops. The residual of the equation at each point was then calculated. Figure 6.2 gives the distributions of the terms in the region of the corner for a sudden contraction with a diameter ratio of 2.50 at $r^{*}=0.1$. For the convenience of the presentation, these data have been normalized with the value of the pressure term at the exit. It is interesting to note that at $R e_{d}=10$ (Figure $6.2(\mathrm{~A})$ ), viscous effects are much larger than inertial effects locally and pressure changes are caused mainly by viscous forces. The presence of parallel lines for the viscous and pressure terms implies that the velocity profile and the pressure gradient are both constant, hence the flow is fully developed. For the high Reynolds number case shown in Figure $6.2(\mathrm{~B})\left(R e_{d}=1000\right)$, the flow remains undeveloped in a larger part of the pipe. Here inertial effects retard the development of the flow because the contraction has caused a considerable amount of the pressure energy to be converted to kinetic energy in the secondary flow. The energy present in the undeveloped profile must be dissipated to the wall as the profile develops. Most importantly, the magnitude of the residual is shown to be small in most cases so far

as this research is concerned. At higher Reynolds number the residual increases, but is still small relative to the dominant terms. This observation suggests that the present procedure for solving for the pressure is valid. 


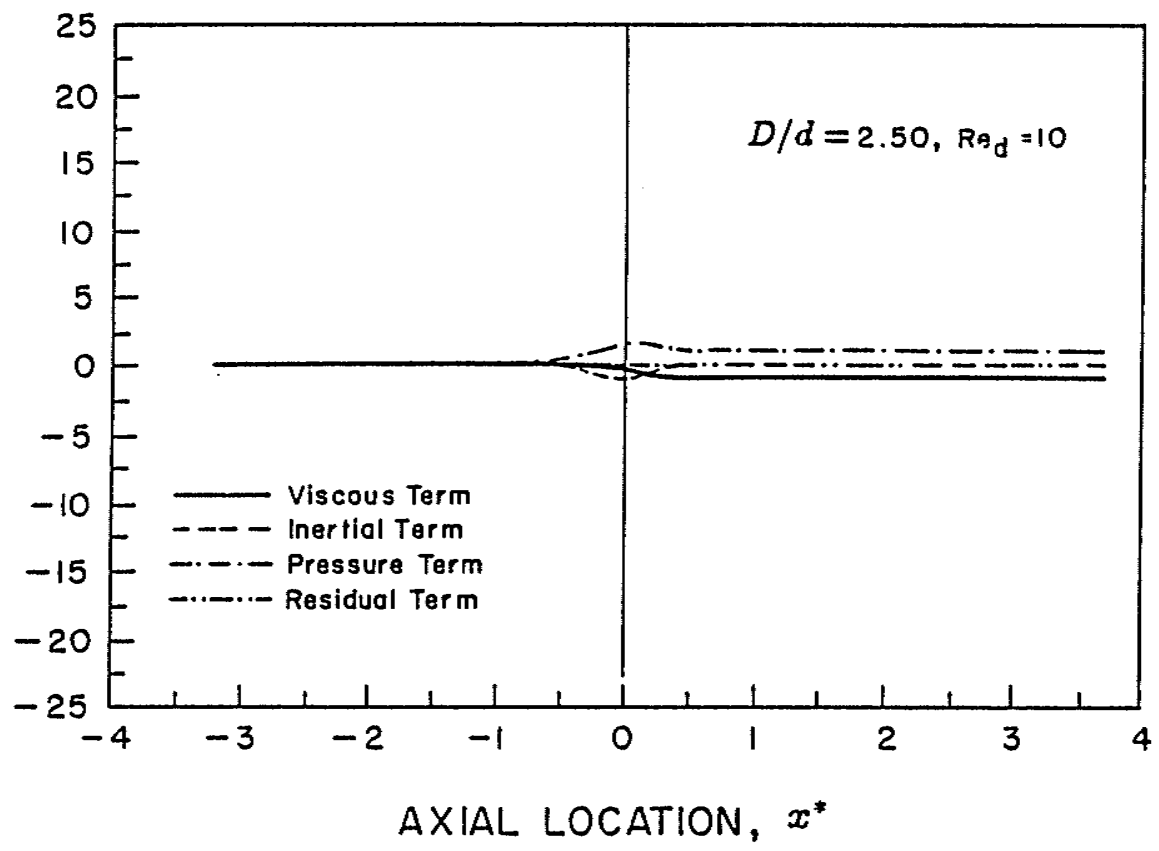

(A). $R e_{d}=10$

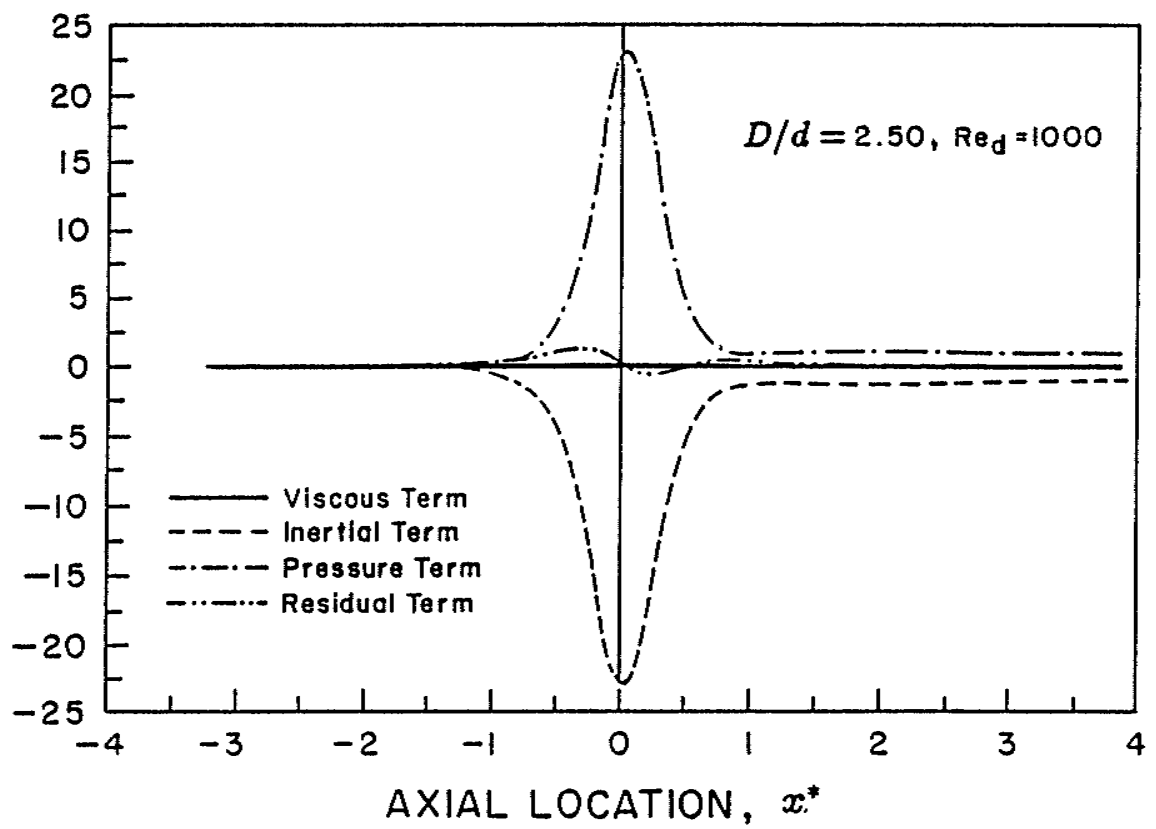

(B). $R e_{d}=1000$

Fig. 6.2. Variations of the terms in Eq. (4.1). 
It is essential that the boundary gradient of the pressure solutions should be compatible with the source term, $S_{p}^{*}$, of the Poisson pressure equation (4.8). This compatibility is ensured if the Green's theorem

$$
\oint_{\partial A^{*}} \frac{\partial p^{*}}{\partial n} d s^{*}=\iint_{A^{*}} S_{p}^{*} d A^{*},
$$

where $A^{*}$ is the solution domain with boundary $\partial A^{*}$, is satisfied (Roache, 1972; Briley, 1974; Ghia et al., 1979).

To evaluate the accuracy of the pressure solutions, both sides of Equation (6.3) have been numerically integrated for different cases. It was found that the difference increases at high Reynolds numbers $\left(R e_{d}>200\right)$ because of the truncation error and the discrepancy comes mostly from the pressure gradient computations in the immediate vicinity of the sharp corner. For the present analysis of high $\mathrm{F}$-number flow in porous media, however, the pore Reynolds number is well below 200. Therefore, it is reasonable to believe that the pressure computation scheme predicts reliable field quantities.

\subsubsection{Accuracy Confirmation of the Numerical Results}

In order to test accuracy of the numerical scheme, the flow in a straight pipe $(D / d=1)$ has been taken as an ideal model to check the correctness and the accuracy of the computer program. By using $21 * 151$ uniform grids, the maximum deviation of the velocity from the Hagen-Poiseuille solution is less than $10^{-3}$, and the relative pressure difference is not larger than $10^{-2}$ for all cases.

By assuming that a steady periodic solution exists, namely, the velocity (or vorticity) field repeats itself in a succession of cross sections that are separated from each other by the wave length $L^{*}$, the exit profiles obtained from the $(m-1)$ th 
iteration are substituted as approximations of the entrance values for the $m$ th iteration in the diverging-converging flow computations. This procedure is continued until the difference of the two profiles at entrance and exit is satisfactorily small. The numerical results show that this process converges rapidly at low Reynolds numbers $\left(R e_{d}<50\right)$. When $R e_{d}>50,4-7$ iterations are needed to obtain a change in the wall vorticity values of less than $10^{-3}$.

A continuity check was performed at every cross section from entrance to exit for all computations to ensure that the mass flow rate is a constant throughout the capillary tube.

In addition to the continuity check, another accuracy test has been carried out. To do this, the following integral momentum equation for the flow in the RUC is derived (see, e. g., Fox and McDonald, 1985):

$$
\begin{aligned}
-\int_{A_{f f}^{*}} p^{*} \cos (\mathbf{n}, \mathbf{i}) d A^{*}= & -\frac{1}{R e_{d}} \int_{A_{f_{o}^{*}}^{*}}\left(\frac{\partial u^{*}}{\partial r^{*}}\right) \cos (\mathbf{n}, \mathbf{j}) d A^{*}+ \\
& +\int_{A_{f o}^{*}} p^{*} \cos (\mathbf{n}, \mathbf{i}) d A^{*}
\end{aligned}
$$

Here $\mathbf{n}$ is the unit outward normal vector of the RUC, $(i, j)$ are the unit vectors in the $\left(x^{*}, r^{*}\right)$ coordinate directions, respectively, $A_{f s}^{*}$ is the fluid/solid interface, and $A_{f f}^{*}$ is the area of entrance and exit of the unit cell.

After the velocity and pressure fields are achieved, both sides of Equation (6.4) can be numerically integrated. They are not exactly equal due to computation errors. However, the difference of the two sides is less than $5 \%$ in all cases.

\subsection{Convergence of the Solutions}

As already mentioned, the finite-difference grids utilized in this study are non- 
uniform in both the axial and radial coordinate directions. The optimum grid was obtained by step-size numerical experiments for different numbers of grid points $(45 * 166,41 * 151$ and $37 * 136)$ to ensure that the vorticity profiles in the corner region are grid independent for flow conditions considered in this study. A sample of the wall vorticity distributions are given in Figure 6.3 for an RUC with $\frac{D}{d}=2$ and $\frac{L_{t}}{L_{p}}=2$. It can be seen that a satisfactory convergence has been achieved by a grid $41 * 151$ for $R e_{d}=100$. Similar situations exist for other Reynolds number cases. Therefore, in most computations, $41 * 151$ grids were used. But for the cases of $R e_{d}>60$ and RUCs' with large diameter ratios $(D / d)$ and small length ratios $\left(L_{t} / L_{p}\right), 45 * 166$ grids were used. It should be pointed out that because the computational grids are non-uniform, the actual grid size in the region of the corners are decreased almost by half for different grid numbers.

The vorticity and stream function equations (4.4) and (4.7) are discretized by means of common central-difference formulas and the approximating sets of difference equations are then solved by the Gauss-Seidel iteration technique. The vorticity values at the neighboring points of the convex corners are obtained by the scheme presented in Section 5.2. The vorticity value at the point $S$ (Figure $5.1)$ is used to define the normalized residual

$$
\varepsilon_{S}=\left|\frac{\left(\Omega_{S}^{*(m)}-\Omega_{S}^{*(m-1)}\right)}{\Omega_{S}^{*(m)}}\right|
$$

because this point is always the last to converge. Here $m$ is the iteration number. A value of $\varepsilon_{S}=10^{-3}$ was used as a convergence criterion in the computations. The characteristics of the convergence are shown in Figure 6.4 for two Reynolds number cases of a sudden contraction flow. It is apparent that as the Reynolds number increases, more iterations are required.

The pressure equation (4.8) is solved by the hybrid method introduced in 


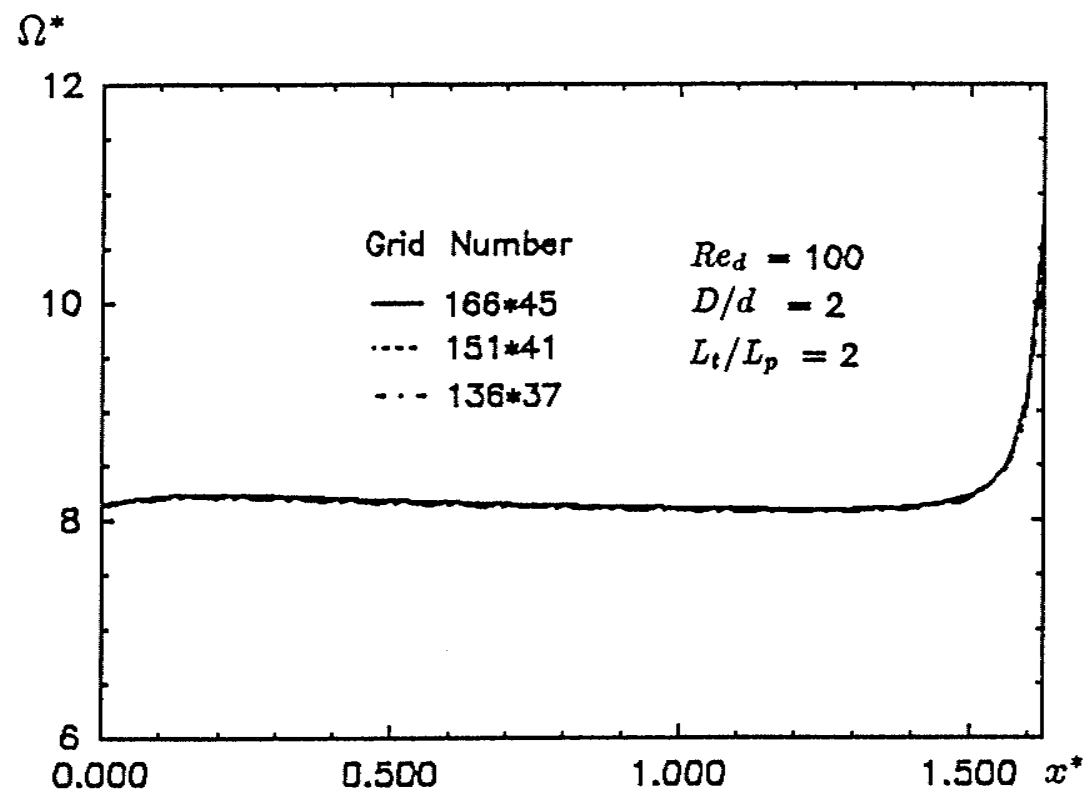

(A). the upstream small pipe.

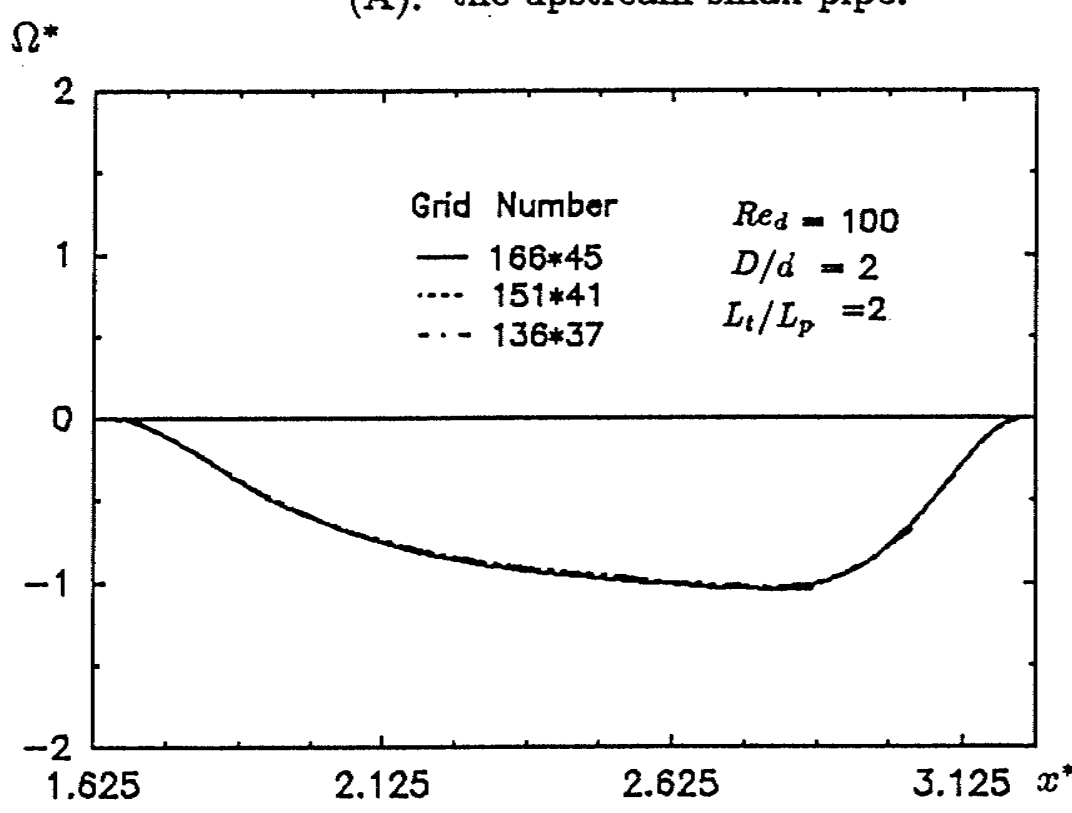

(B). the large pipe.

Fig. 6.3. Wall vorticity distributions in an RUC for various grids. 


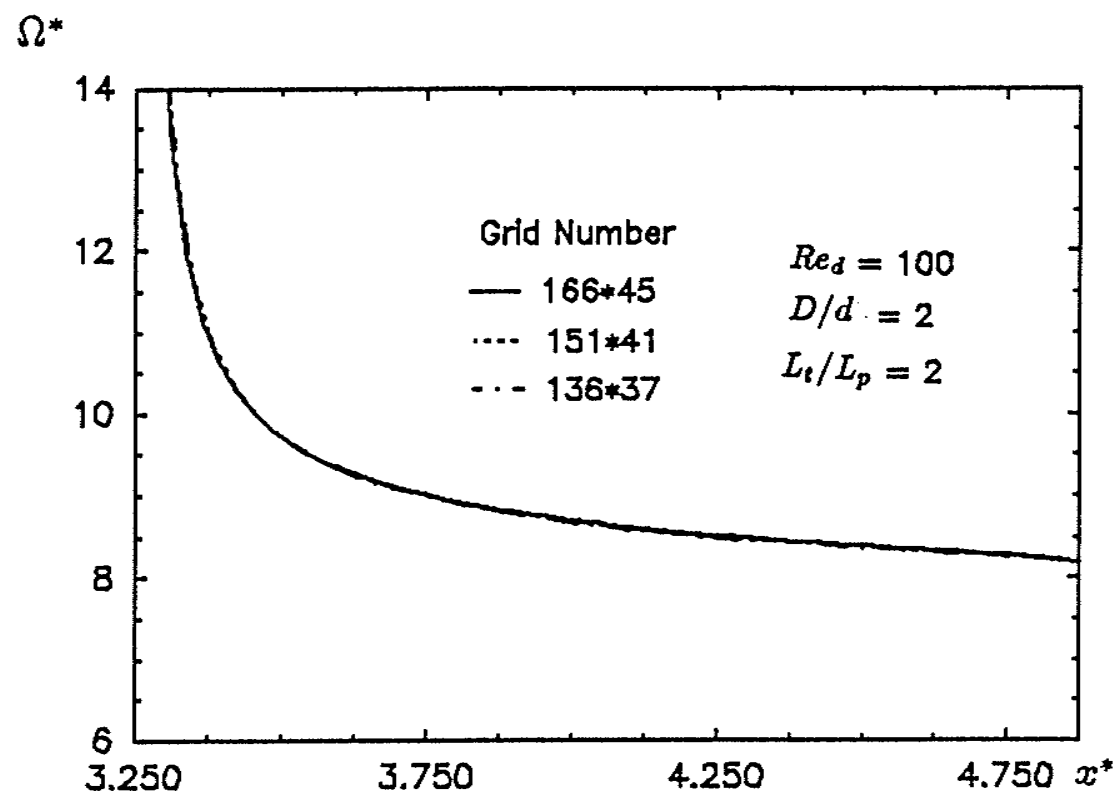

(C). the downstream small pipe.

Fig. 6.3. Wall vorticity distributions in an RUC for various grids. 


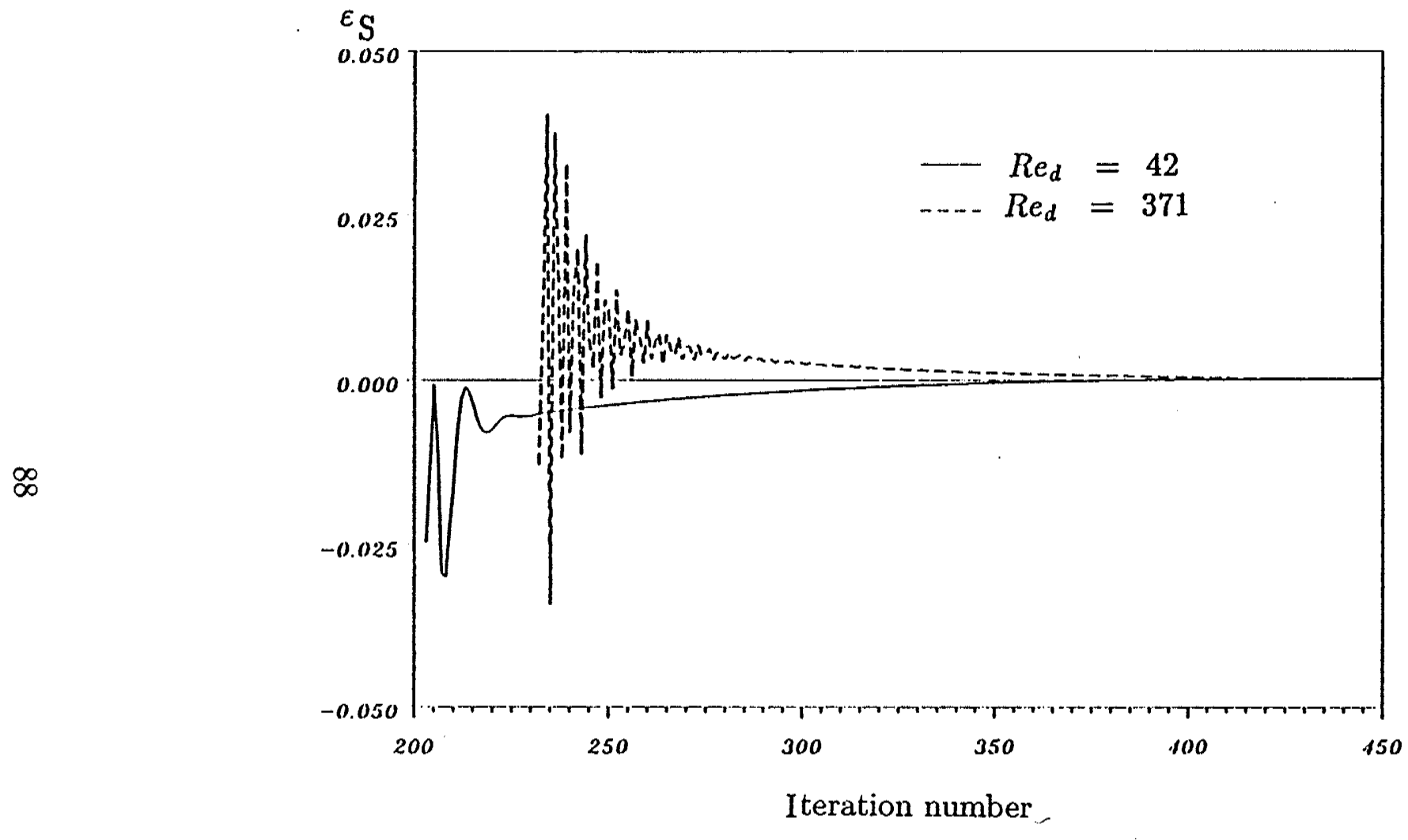

Fig. 6.4. The converging characteristics of the vorticity-circulation method. 
Section 5.2 and the SOR technique is employed in the numerical solving process. The numerical results confirmed that within $200-300$ iterations, the normalized error function

$$
\varepsilon_{p}=\left|\frac{\left(p^{*(m)}-p^{*(m-1)}\right)}{p^{*(m)}}\right|
$$

over the whole calculation domain in all cases satisfied

$$
\varepsilon_{p} \leq 1 \times 10^{-4}
$$

\subsection{Computer Program}

The computer program used to obtain the numerical solution of the problem is written in standard FORTRAN 77. To avoid unnecessary repetition and minimize the computer time and the amount of storage, the simulation was implemented in three separate programs named as GRID, VORSTR and PRESS.

The program GRID is used to generate the non-uniform computational grids in the solution domain of different geometries. The geometrical parameters which are required by the subsequent calculations, such as local grid sizes and the $\left(x^{*}, r^{*}\right)$ coordinates of the grid points, can be determined using GRID and stored in the corresponding two-dimensional arrays.

With the computational grids established, the program VORSTR is then utilized to iteratively determine the vorticity-stream function fields as well as velocity distributions in a diverging-converging RUC. These quantities will afterwards serve as the input for subsequent pressure computations.

The program PRESS has been designed to recover the pressure field using the hybrid scheme presented in Section 5.2 and to determine the macroscopic 
coefficients, such as the permeability $(k)$ and the inertial coefficient $(\beta)$ which have been derived in Chapter 3 in terms of microscopic quantities, through relevant numerical integrations. 


\section{CHAPTER 7}

\section{FLOW MEASUREMENTS}

The experiments described in this chapter were designed mainly for examining the accuracy of the pressure computational scheme. For this purpose, the experimental work in this study has been devoted to the problem of flow in a tube with a sudden contraction which embodies most aspects of the characteristics of the present diverging-converging RUC flow in terms of the flow analysis and numerical simulation. Furthermore, the pressure-flow rate relationship observed in this test will also be useful in clarifying the confusion on the excess pressure drop of a sudden contraction flow.

\subsection{Introduction}

Because of its considerable importance to both theoretical analysis and engineering applications, incompressible viscous flow from a large pipe (or a reservoir) into a small pipe constitutes one of the most widely studied problems of fluid dynamics. A number of numerical studies of the problem of a sudden contraction have been published. One of the most comprehensive early studies is that of Vrentas and Duda (1973). Another previous contribution to our knowledge of the subject is the study by Durst and Loy (1985). That work presents both laser doppler anemometry measurements and numerical computations of the velocity fields which have been used to verify the accuracy of the present numerical profiles in Chapter 5 . Surprisingly, few computational and experimental results have been published on 
the pressure distributions caused by a contraction. In fact, only two comprehensive data sets are available for the excess pressure loss of a sudden contraction flow (Astarita and Greco, 1968; Sylvester and Rosen, 1970) and these data sets show major disagreement with each other and with previous asymptotic limits.

A major interest in such flow is associated with the ability to predict the pressure drop between any two axial locations. To characterize the flow development in the region, the excess pressure drop, $\Delta p_{e}^{*}$, which is defined as the difference between the actual pressure drop in the system and the pressure drop that would exist if only losses from fully developed flow were present in both pipes, has been utilized in the analysis of such flow. Through a dimensional consideration, Holmes (1967) proposed an expression for the excess pressure drop, and this expression can be written in the form

$$
\Delta p_{e}^{*}=\kappa+\frac{\kappa^{\prime}}{R e_{d}}
$$

In Equation (7.1), $\kappa$ and $\kappa^{\prime}$ are two dimensionless parameters and have frequently been referred to as the Hagenbach and Couette corrections, respectively. A variety of methods have been employed for the determination of these parameters. Weissberg (1962) considered the problem of Newtonian flow into or away from an infinite reservoir in the limit as $R e_{d} \rightarrow 0$. This analytical result provides an useful, low Reynolds number upper bound for the excess pressure drop and is in good agreement with the experimental data of La Nieve and Bogue (1968) and Holmes (1967). Various numerical solutions have been derived in order to provide information relating the flow development to pressure drop in the region of sudden changes in the cross sectional area. Most of these studies concentrated on large diameter ratios and employed the boundary layer assumptions, thus neglecting the axial transport of vorticity and assuming that pressure is a function of axial distance 
only (Sparrow et al., 1964; Collins and Schowalter, 1963; Vrentas et al., 1966; Christiansen and Lemmon, 1965; Campbell and Slattery, 1963). These results are limited to high Reynolds number entrance flow and in some sense provide a high Reynolds number asymptote to a more complete solution of the problem. In recent years, some effort has been directed towards obtaining flow predictions by solving the full Navier-Stokes equations for various contraction ratios and Reynolds numbers (Vrentas and Duta, 1973; Carter, 1969; Durst and Loy, 1985). However, due

to the difficulties in extracting reliable pressure distributions (Ref. Section 5.2), insufficient data of both the pressure field and the excess pressure drop have been provided in these studies.

In this research, the measurements of the relationship between pressure drop and flow rate were conducted in the experimental facility introduced in Section 7.2. The measured values, the wall static pressure distribution and the excess pressure drop, will be used to compare with the numerical predictions. A thorough comparison between the experimental results and the numerical values predicted by the hybrid method (Section 5.2) and other published data are given in Section 7.4 .

\subsection{Experimental Apparatus}

In the present research, the measurements of the relationship between the pressure drop and the flow rate (the Reynolds number) were conducted for two contractions with diameter ratios of 8.0 and 2.5 using an experimental facility introduced in this section. To achieve accurate measurements, extra caution has been exercised in the fabrication of the facility and in the procedure of the tests. 


\subsubsection{Geometry of the Sudden Contraction Test Section}

Two sudden contraction pipes with diameter ratios of 8.0 and 2.5 were carefully manufactured in the porous media laboratory at the University of Manitoba. Each sudden contraction test section consists of two different pipes which are referred to the upstream and downstream pipe in this chapter. The dimensions of each pipe are listed in Table 7.1. The lengths of both upstream and downstream pipes have been kept long enough to make sure that the fully-developed flow condition is fulfilled for the tests in this study.

\begin{tabular}{|c|c|c|}
\hline & Table 7.1. Dimensions of the Test Sections (in mm) \\
\hline & Large pipe & Small pipe \\
\hline$D / d=2.5$ & diameter: $15.37 \pm 0.381$ & diameter: $6.35 \pm 0.254$ \\
\cline { 2 - 3 } & length: 1,854 & length: 1.854 \\
\hline$D / d=8.0$ & diameter: $50.80 \pm 0.762$ & diameter: $6.35 \pm 0.254$ \\
\cline { 2 - 3 } & length: 1,854 & length: 1.854 \\
\hline
\end{tabular}

Transparent acrylic pipes were used in the fabrication of the sudden contraction test sections. Each test section consists of two different diameter pipes which are joined by two $6 \mathrm{~mm}$ thick acrylic flanges. To ensure the concentricity of the test sections, all the flanges were machined at same time in a numerical controlled milling machine with a precision bore hole in the center. For each pipe, the connecting end were machined flush and glued inside of the flange, so that when the two flanges were bolted together a sharp edged contraction was formed.

The pressure tap holes of $1.2 \mathrm{~mm}$ diameter were distributed along the length of the pipe. The axial locations of these pressure taps are given in Table 7.2.

Table 7.2. Arial Locations of the Pressure Taps (in $\mathrm{mm}$ )

\begin{tabular}{|c|c|c|c|c|c|c|c|c|c|c|c|c|}
\hline Tap number & 1 & $i$ & 3 & 4 & 5 & 6 & 7 & 8 & 9 & 10 & 11 & 12 \\
\hline Avial Location & -53.3 & $41-40.6$ & 33.02 & -25.40 & -20.32 & -15.2 & -10.16 & -5.08 & -2.34 & +1.27 & +5.08 & -10. \\
\hline Tap number & 13 & 14 & 15 & 16 & 17 & 18 & 19 & 20 & 21 & 22 & 23 & \\
\hline
\end{tabular}




\subsubsection{Test Facility}

The pressure distributions across the test section were measured as a function of flow rate by means of the apparatus shown in Figure 7.1. The overhead tank with 180 liter capacity was found convenient to use in furnishing constant head. The flow rate was easily controlled either by adjusting the height of the overhead tank which was installed in a frame and could be moved up and down by a motor or by changing the relative level of the outlet tubing located in the storage tank. A $70 \mathrm{~mm}$ ID overflow line on the overhead tank ensured that a constant level and thus a constant pressure head was maintained at the inlet to the test section. At the bottom of the overhead tank, an opening was connected to the test section by a vinyl tube (20 $\mathrm{mm}$ ID). The working fluid was circulated around the closed loop by a centrifugal pump with a reliable speed drive.

Samples of the working fluid were collected for a timed portion of the tube effluent and weighed on an electronic analytical balance with an accuracy of $\pm 0.01 \mathrm{~g}$, and the flow rate was then calculated.

The pressure distributions were measured in a series of $3.17 \mathrm{~mm}$ ID acrylic upright tubes (see Figure 7.1) which were connected to wall static pressure taps. The heights of the liquid in each tube were read by means of a Sargent-Welch cathetometer, with a vernier reading to $\pm 0.05 \mathrm{~mm}$. To have adequate measuring readings at different flow rates, three Newtonian liquids were used as working fluids in the tests. They are water, and $40 \%$ and $60 \%$ glycerine in water solutions.

\subsection{Experimental Accuracy}

To obtain reliable experimental results, several measures have been adopted to 


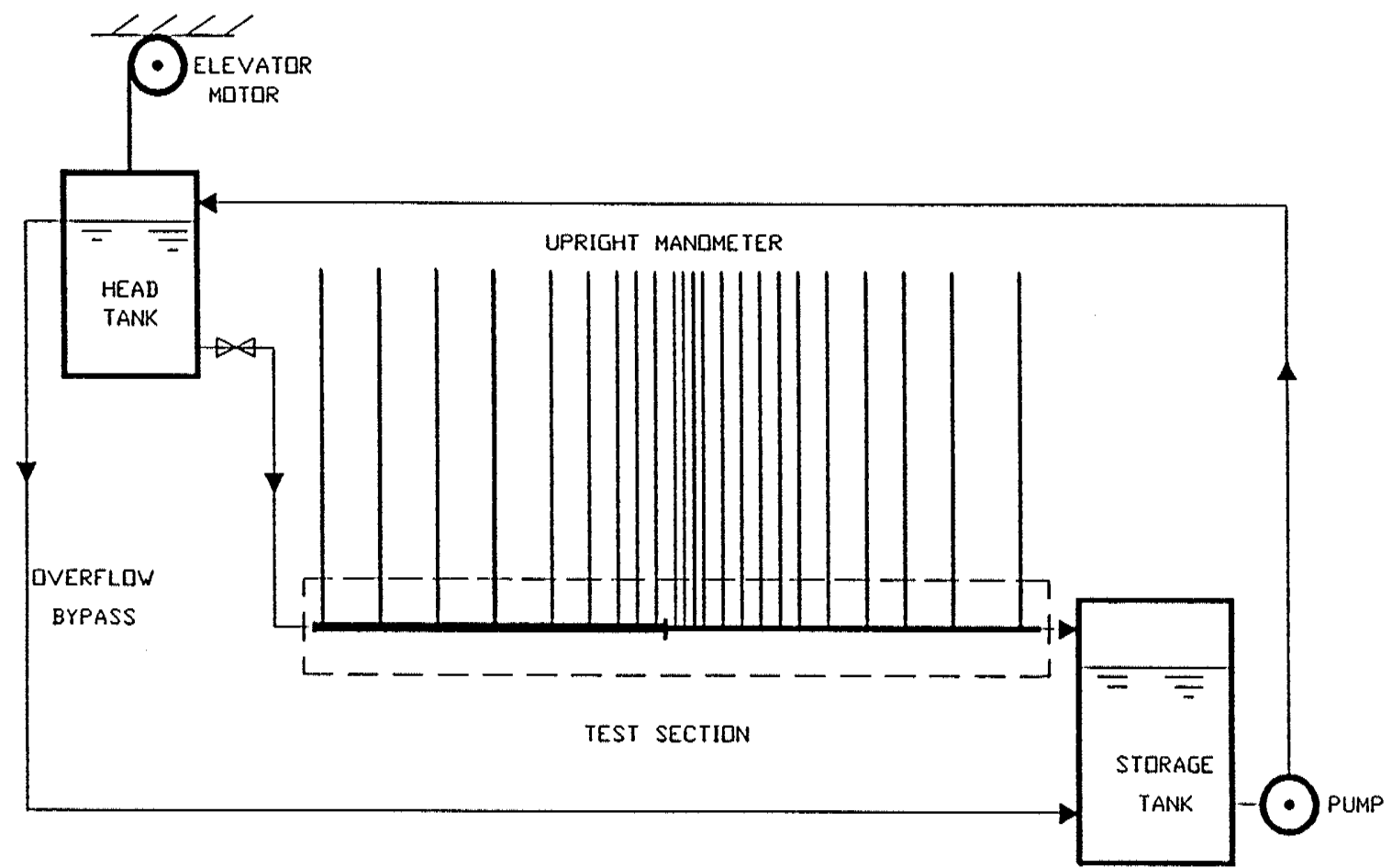

Fig. 7.1. Apparatus used to measure pressure distributions across the contraction test section. 
minimize the possible errors introduced by the experimental setup and in the experimental procedure.

First of all, the cathetometer was adjusted to be oriented in a horizontal plane using the build-in level, and this level orientation was confirmed by monitoring the heights of the liquid in the upright tubes when there was no flow in the test section. The test section was then rechecked to ensure that its whole length was located in a horizontal plane.

As explained earlier, the flow rate to the test section was regulated through the relative levels of the overhead tank and the outlet tube. To ensure a stable flow rate for each condition change, the tests were carried out after a sufficient period of time such that the difference of the flow rates evaluated from two collected samples were within $1 \%$.

To achieve accurate measurements, trapped air bubbles in the test section and in the upright manometer were evacuated by various techniques from vacuum pump to manual suction.

It is obvious that any significant change of the fluid temperature could have introduced an error in the experimental data. Due to the long length of the test section, it was difficult to keep the fluid temperature constant in the whole flowing process simply by heating the fluid in the storage tank (Sylvester and Rosen, 1970). Therefore, all the tests in the present study have been performed under the condition that the room temperature and the fluid temperature are the same. This constancy of the fluid temperature was guaranteed by recording the room temperature and the temperatures at several points in the flow loop simutaneously during each run. The tolerance for temperature change was $0.2^{\circ} \mathrm{C}$. 
The estimates of the experimental uncertainty were obtained following the procedures of Kline and McClintock (1953). A 95\% confidence level for the uncertainty of the excess pressure drop, $\Delta p_{e}^{*}$, was not more than $6 \%$ depending on flow conditions. For the Reynolds number, the overall uncertainty was less than $5 \%$. A sample calculation for Reynolds number uncertainty is presented in APPENDIX E.

\subsection{Result Analysis and Comparison}

\subsubsection{Data Reduction}

The primary goal of the experimental investigation was the determination of the wall pressure distributions and the excess pressure drops for fluid flow through a sudden contraction. The independent parameters were the Reynolds number and the diameter ratio for the geometry considered. In the presentation of the data, the cross-sectional area of the small pipe is used as the reference area. The mean velocity and the diameter of this cross-section has been utilized as the velocity and length scales for nondimensional quantities.

The mass flow rate in the small pipe, $\dot{m}$, is determined from

$$
\dot{m}=\frac{W}{\mathrm{~g} \Delta t}
$$

where $W$ represents the weight of the fluid collected in a time interval, $\Delta t$, and $\mathbf{g}$ represents the acceleration of gravity.

For a given geometry, the pressure drop depends only on the Reynolds number

$$
R e_{d}=\frac{\rho \bar{U}_{d} d}{\mu}=\frac{4 \dot{m}}{\pi \mu d}
$$


The dynamic viscosities of the fluid were rechecked by using a HAAKE falling-ball viscometer at the same temperature conditions as the tests being performed.

The wall static pressure, $p$, is presented in a dimensionless form

$$
p^{*}=\frac{p}{\rho \bar{U}_{d}^{2}}=\frac{g h}{\bar{U}_{d}^{2}}=\left(\frac{\rho \pi d^{2}}{4 \dot{m}}\right)^{2} g h,
$$

where $h$ is the height of the upright manometer, taking Tap No. 1 (see Table 7.2) as the reference level.

After the static pressure distributions are determined, the excess pressure drop as defined in Equation (7.1) can be written as

$$
\Delta p_{e}^{*}=\frac{\Delta p_{e}}{\frac{1}{2} \rho \bar{U}_{d}^{2}}=\Delta p^{*}-\Delta p_{f u l l y d e v .}^{*},
$$

where $\Delta p^{*}$ is the dimensionless pressure drop through the contraction, and $\Delta p_{\text {fully dev. }}^{*}$ is the nondimensional pressure drop due to a fully developed flow calculated from

$$
\Delta p_{\text {fullydev. }}=\frac{64}{R e_{d}} L_{d}^{*}\left[\frac{L_{D}^{*}}{L_{d}^{*}}\left(\frac{d}{D}\right)^{4}+1\right] .
$$

In Equation (7.6), $L_{d}^{*}$ and $L_{D}^{*}$ denote the dimensionless lengths of the small and large pipes, respectively.

\subsubsection{Pressure Distribution}

The pressure drops were measured for a range of Reynolds numbers $\left(10 \leq R e_{d} \leq\right.$ 1800 ) for each of the contractions. It has been found in the tests that the viscous pressure losses in the large pipe (upstream of the contraction) were negligibly small for the geometries considered. This observation suggests that the viscous integrals over the surface of the large pipe in Equations (3.36), (3.37) and (3.38) may safely 
be ignored in the evaluation of the macroscopic coefficients. This point will be brought up in more detail in Chapter 8.

Some representative pressure profiles in the small pipe (downstream of the contraction) are shown in Figure 7.2 and Figure 7.3 with $p^{*}=0$ being assigned to the pressure level at the first tap in the large pipe of the test section. For the case of $D / d=8.0$, computed pressure profiles of fully developed flow are also plotted (Figure 7.2). It can be seen that the Hagen-Poiseuille flow condition has been established within the length of the small pipe for the Reynolds numbers in this study. A comparison between the numerical profiles predicted by the hybrid pressure computational method (Section 5.2) and the measured values are given in Figure 7.3 for the entrance region. Curves are for $R e_{d}=12.2,80,128.7$ and 652 , respectively. A good agreement between measurements and computations may be observed. This comparison verifies that the pressure integrations over the fluid/solid interface predict reliable boundary values for subsequent iterative pressure computations of the interior points by means of the procedure explained in Section 5.2 .

\subsubsection{Comparisons of the Excess Pressure Drop}

The excess pressure drop, $\Delta p_{e}^{*}$, which physically represents the kinetic energy changes and the excess viscous dissipation of energy in the flow development, was calculated for various Reynolds numbers. The dependence of excess pressure drop on Reynolds number is shown in Figure $7.4(D / d=8.0)$ and Figure $7.5(D / d=$ 2.5). It can be seen that for $D / d=8.0$ an increase in the excess pressure drop occurs below $R e_{d} \approx 150$. A similar observation has been reported by Astarita and Greco (1968). According to the experimental data, the best estimates for 


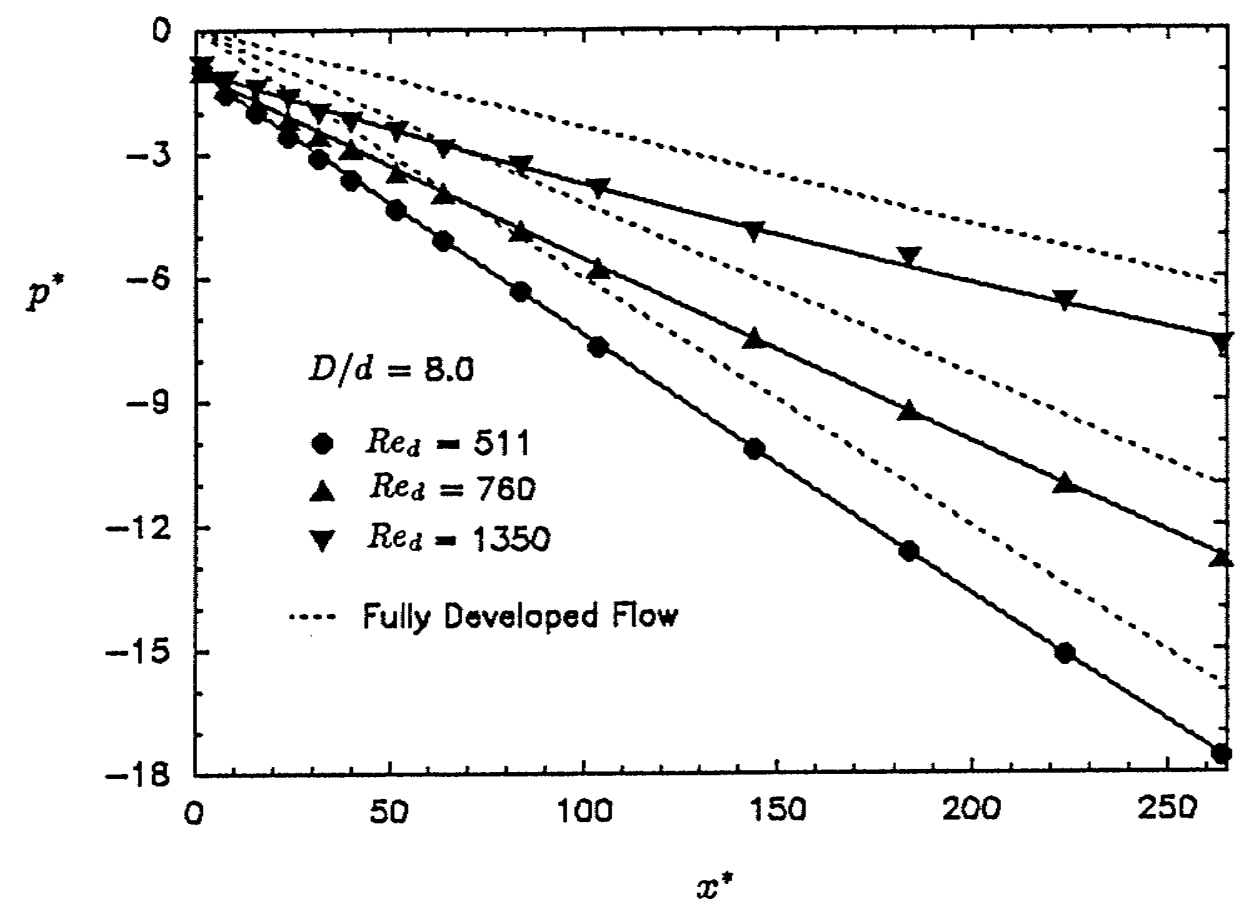

Fig. 7.2. Representative pressure distributions in the small pipe of a sudden contraction. 


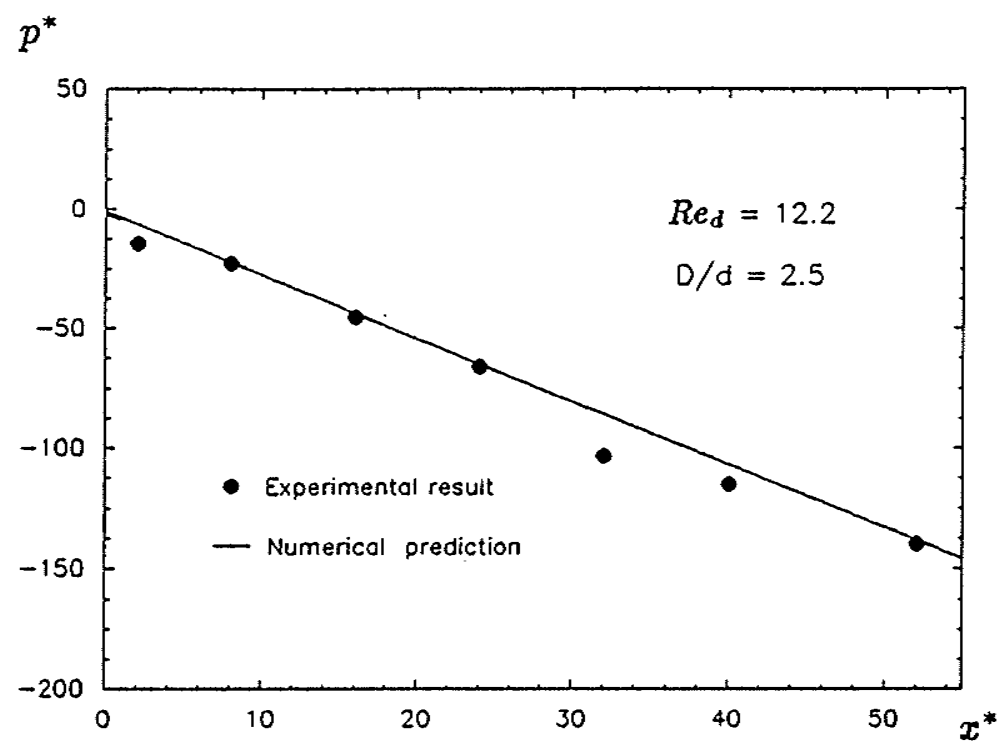

(A). $R e_{d}=12.2$

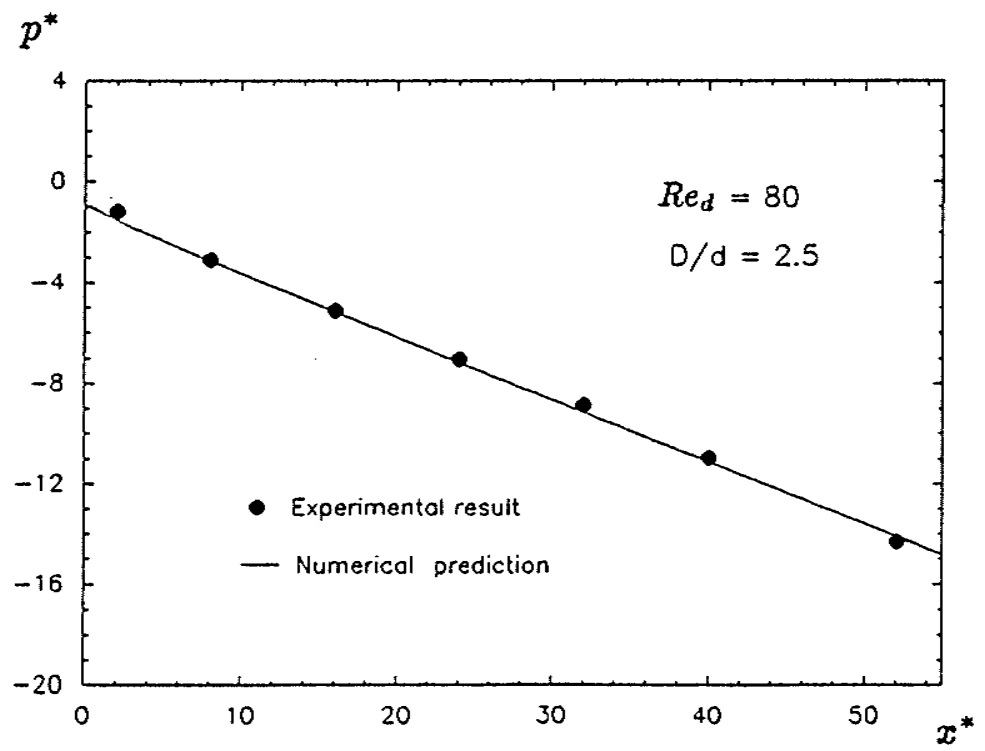

(B). $R e_{d}=80$

Fig. 7.3. Comparison of wall pressure distributions in the small pipe of a sudden contraction flow. 

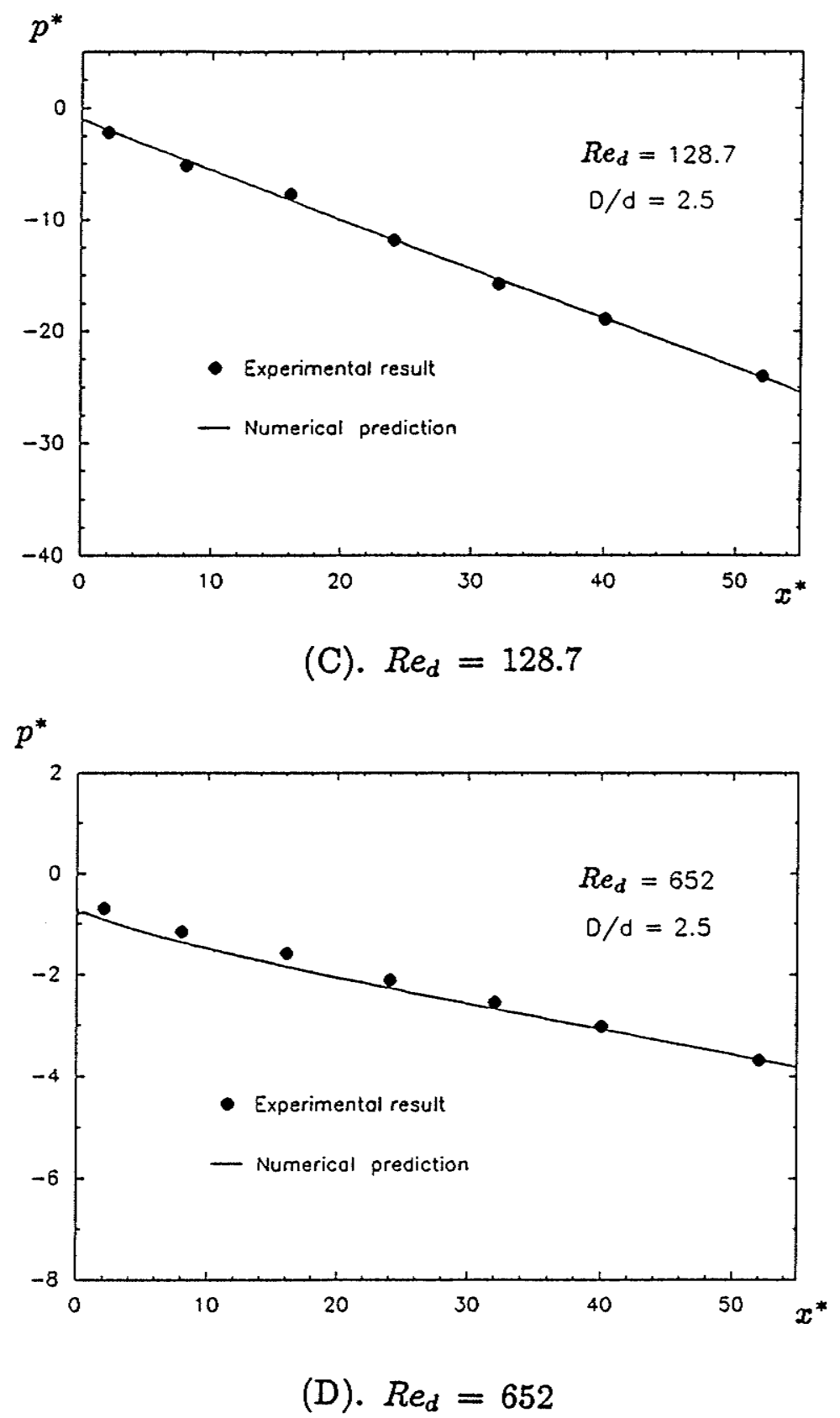

Fig. 7.3. Comparison of wall pressure distributions in the small pipe of a sudden contraction flow. 


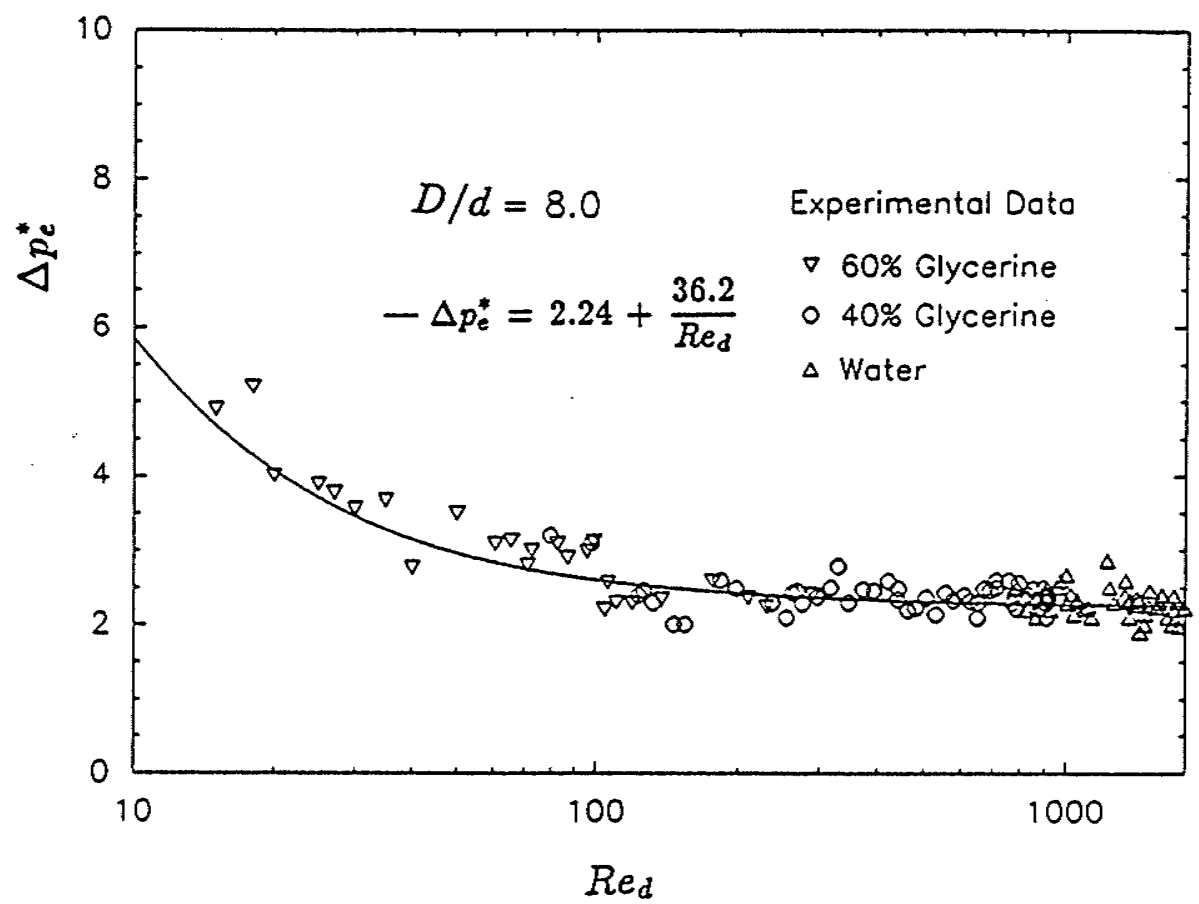

Fig. 7.4. The excess pressure drop through a sudden contraction, $D / d=8.0$. 


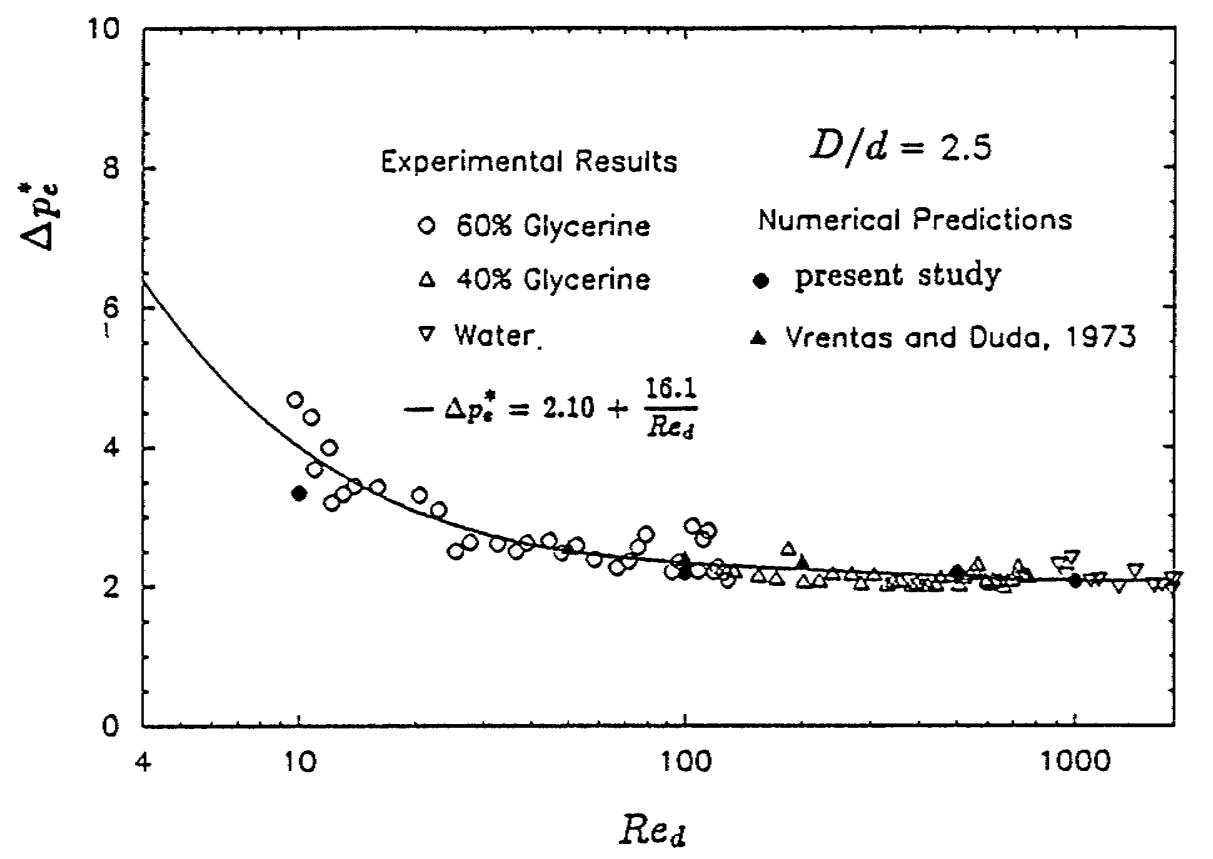

Fig. 7.5. The excess pressure drop through a sudden contraction, $D / d=2.5$. 
Hagenbach and Couette corrections are $\kappa=2.24$ and $\kappa^{\prime}=36.2$ for $D / d=8.0$ as shown in Figure 7.4. For the purpose of comparison, the numerical results predicted by the hybrid method as well as the values given by Vrentas and Duda (1973) have been plotted in Figure 7.5. The present numerical results compare favorably with both of them. The solid line drawn in Figure 7.5 is Equation 7.1 with $\kappa=2.10$ and $\kappa^{\prime}=16.1$.

Comparisons of the Hagenbach and Couette corrections of the present with some results from previous experimental and theoretical studies are given in Tables 7.3 and 7.4. It is quite clear that both $\kappa$ and $\kappa^{\prime}$ are $\frac{D}{d}$ dependent. In the low $R e_{d}$ range (Table 7.4), both experimental and numerical results of the present study fall below the upper bound theoretically established by Weissberg (1962) and experimentally verified by La Nieve and Bogue (1968) for the "creeping" flow limit, and a consistent trend holds as $\frac{D}{d}$ increases from low to high values. The values at high $R e_{d}$ (Table 7.3) also agree well with other numerical results and theoretical limit (boundary layer calculations for $\frac{D}{d} \rightarrow \infty$ ). The experimental results of both Astarita and Greco (1968) and Sylvester and Rosen (1970) lie considerably above the present results and these two data sets agree neither with each other nor with the asymptotic limits. Through an analysis of the hydrodynamic definitions of the corrections ( $\kappa$ and $\kappa^{\prime}$ ) derived by a momentum conservation, Ma and Ruth (1993c) pointed out that the negligence of the pressure change at the cross section of the sudden contraction might result in the higher values of Hagenbach correction. Another possible reason for the deviation of the experimental results by Astarita and Greco (1968) and Sylvester and Rosen (1970) is the temperature change in the flow loop which affects considerably the accurate determination of these corrections. 
Table 7.3. Comparison of the Hagenbach Correction ( $\kappa$ )

\begin{tabular}{|c|c|c|c|c|}
\hline Reference & Type of Solution & $\frac{D}{d}$ & $\kappa$ & $R e_{d}$ Range \\
\hline Rieman (1928) & Experimental & $\infty$ & 2.25 & $200-1000$ \\
\hline Astarita \& Greco (1968) & Experimental & 2.5 & 5.48 & $10-2000$ \\
\hline Sylvester \& Rosen (1070) & Experimental & 8.0 & 2.40 & $6-2000$ \\
\hline Campbell \& Slattery (1963) & Numerical & $\infty$ & 2.09 & \\
\hline Collins \& Schowalter (1963) & Numerical & $\infty$ & 2.33 & boundary layer \\
\hline Vrentas et al.(1966) & Numerical & $\infty$ & 2.28 & assumptions \\
\hline Sparrow et al.(1964) & Numerical & $\infty$ & 2.24 & \\
\hline \multirow[t]{5}{*}{ present Research } & Experimental & 8.0 & $\underline{2.24}$ & $10-1800$ \\
\hline & & 2.5 & $\underline{2.10}$ & $10-1800$ \\
\hline & Numerica!* & 2.5 & $\underline{2.08}$ & $0.1-1000$ \\
\hline & & 1.67 & $\underline{1.89}$ & $0.1-1000$ \\
\hline & & 1.25 & 1.31 & $0.1-1000$ \\
\hline
\end{tabular}

* Reported in Ma and Ruth (1993a).

Table 7.4. Comparison of the Couette Correction $\left(\kappa^{\prime}\right)$

\begin{tabular}{lcccc}
\hline \multicolumn{1}{c}{ Reference } & Type of Solution & $\frac{D}{d}$ & $\kappa^{\prime}$ & $R e_{d}$ Range \\
Dorsey (1926) & Experimental & $>50$ & $18.37-30.67$ & $<10$ \\
Holmes (1967) & Experimental & $>50$ & 37.7 & $0.01-1$ \\
La Nieve \& Bogue (1968) & Experimental & 11.8 & 36.8 & $6.75 \times 10^{-4}$ \\
Astarita \& Greco (1968) & Experimental & 2.5 & 795 & $10-2000$ \\
Sylvester \& Rosen (1970) & Experinental & 8.0 & 295 & $6-2000$ \\
Weissberg (1962) & Numerical & $\infty$ & 37.7 & $R e_{d} \rightarrow 0$ \\
Present Research & Experimental & 8.0 & $\underline{36.2}$ & $10-1800$ \\
& & 2.5 & $\underline{16.1}$ & $10-1800$ \\
& Numerical* & 2.5 & $\underline{14.41}$ & $0.1-1000$ \\
& & 1.67 & $\underline{10.70}$ & $0.1-1000$ \\
& & 1.25 & $\underline{4.20}$ & $0.1-1000$ \\
\hline
\end{tabular}

* Reported in Ma and Ruth (1993a). 


\section{CHAPTER 8}

\section{RESULTS AND DISCUSSION}

A systematic numerical experimental study was performed by the procedure given in Chapter 5 for the geometric model introduced in Chapter 4 with the aim of improving the understanding of how the macroscopic properties of a porous medium are influenced by the pore geometric and flow conditions. As already described, three parameters characterizing the solution domain of the present RUC's are the diameter ratio, $D / d$, the length ratio, $L_{t} / L_{p}$, and the length to diameter ratio, $L / d$.

By changing any of the three, different flow channels will be formed. Samples of the RUC's used in the computations are given in Figure 8.1 to graphically show the trend of the geometric variations. In this research, the numerical calculations of the diverging-converging capillary flow were furnished for the RUC's formed by changing sequentially the characteristic dimensionless parameters:

Diameter ratio: $D / d=1.5,2,3,4$;

Length ratio: $L_{t} / L_{p}=1.143,2,3.167,4$;

Periodical length to diameter ratio: $L / d=2.925,3.9,4.875,5.363$;

Reynolds number: $R e_{d}=0.01-100$.

In this chapter, the numerical results for the microscopic flow regimes within different RUC's will be presented in Section 8.1, and a full discussion of behaviors of the macroscopic properties will then be given in Section 8.2. Based on these results, Section 8.3 will focus on a quantitative analysis of the nonlinear phenomena 
in high $\mathrm{F}$-number porous media flow.

\subsection{Microscopic Flow Mechanisms}

Developments of the fluid motion for the RUC's with different geometries and pore Reynolds numbers have presented diverse patterns as distinct from simple expansion or contraction flow. The detailed information of the microscopic flow field provided in this section, in association with the quantities from the continuum approach on the macroscopic level (see Section 8.2 ), will be helpful to gain a better knowledge of the nature of the flow in porous media. All the figures are given at the end of the chapter for the continuity of the presentation.

\subsubsection{Streamlines and Vorticity Contours}

The pore flow fields were determined by the numerical procedures developed in Chapter 5 for the different RUC's at various flow rates. Some typical flow patterns in the diverging-converging RUC's are presented in Figures 8.2, 8.3 and 8.4 for changing flow Reynolds number and geometry. In Figure 8.2, the solid curves in the upper portion represent the streamlines and the curves in the lower portion represent the vorticity contours. For the cases of diameter ratio $D / d=2$, two small-size corner vortices and a symmetrical vorticity distribution are found for $R e_{d}=1$ (Figure 8.2(A)) which represents a typical Stokes type of flow. The sizes and positions of the vortices change gradually as $R e_{d}$ increases. The vortex at the back-step wall moves downward and expands as shown in Figure 8.2(B). At even higher $R e_{d}$, these two vortices degenerate into a large recirculation zone which fills the cavity of the large segment of the RUC (Figure $8.2(\mathrm{C})$ ). The vorticity contours 
shown in Figures $8.2(\mathrm{~B})$ and $8.2(\mathrm{C})$ are stretched in the direction of flow by the convective effects as the Reynolds number becomes larger.

The streamlines for geometries of large diameter ratio are given in Figure 8.3(A). A three-vortex system, two small ones at the corners and a large one at the center (Figure 8.3(A)), becomes a two-vortex system with changing sizes and intensities as $R e_{d}$ increases (Figure 8.3(B)). Another typical pattern, shown in Figure 8.4, is observed for large length ratio $\left(L_{t} / L_{p}\right)$ conditions. In such cases, only one observable vortex was predicted for all the Reynolds numbers studied.

The vorticity distributions over the fluid/solid interface with normal in the $r$ direction are given in Figure 8.5 for different RUC's as a function of the Reynolds number. It is obvious that two peaks of vorticity exist at the convex corners for all cases, and the flow erodes the vorticity peak more quickly at lower Reynolds number. The different flow patterns shown in Figure 8.2 can be related to the wall vorticity distributions in Figure $8.5(\mathrm{C})$ where the separation and reattachment points may be identified with $\Omega^{*}=0$.

\subsubsection{Velocity Fields}

The centerline velocity distributions are plotted in Figure 8.6 as functions of the Reynolds number for two RUC's with different length ratios. The position of the minimum moves in the direction of flow and the magnitude of the lowest point goes up as Reynolds number increases (e.g., Figure 8.6(A)). This trend can be better understood by reviewing the flow patterns in Figure 8.2. The development of the vortices narrows the effective flow passage as Reynolds number increases.

Figures $8.7,8.8$ and 8.9 show the numerical axial and radial velocity profiles 
at several representative length positions: the entrance of the RUC, the inlet of the large cylindrical segment, two positions inside the large segment, and the mouth of the second narrow pipe. For the cases of $R e_{d}=20$ and 80 (Figures 8.8 and 8.9), backward flow exists at a certain radial distance in the large segment because of the recirculation region. However, no obvious vena contracta type of behaviour (and velocity overshoot) at high Reynolds number is observed for the cases of the diverging-converging flow. In fact, the development of the vortex system in the diverging-converging pipe weakens the effects of the sudden geometric change and presents the flow as distinct from the single expansion or contraction flow.

A series of velocity vector diagrams for various RUC's is presented in Figures $8.10,8.11$ and 8.12 at different Reynolds numbers from 1 up to 100 . These diagrams may serve as a complement to the results presented above to show variety of flow patterns at different geometric and flow conditions.

\subsubsection{Pressure Distributions}

After the velocity fields were determined, the pressure distributions were calculated by the hybrid scheme (Section 5.2). The cross-sectional pressure profiles at six typical length positions are given in Figure 8.13 for the case of $D / d=3$ at $R e_{d}=$ 80. The dashed lines represent the axial positions where the profiles were taken. The solid lines represent the pressure values above the entrance pressure and as scaled in the graph. It can be seen that most of the pressure loss comes from the narrow pipe, and far from the corner the cross-sectional pressure values change very little although they are never constant.

A representative evolution of the cross-section mean pressure $\bar{P}^{*}$ is shown in Figure 8.14. The solid lines on the graph are the pressure distributions which 
would exist if only fully developed, Hagen-Poiseuille flows were present in the large and the small pipe. For the cases of $R e_{d}=1,10$ and 20 (Figure 8.14(A) (C)), the pressure values in the upstream small pipe are close to those of Hagen-Poiseuille flow because the process is dominated by viscous forces. In the large pipe, the mean pressure gradually goes up depending on the Reynolds number. This trend was observed for all Reynolds numbers studied. The decelerating motion of the fluid leads to an increase in the static pressure on the fore-step wall.

Another dimensionless parameter, the excess momentum loss factor $\xi_{p}$, was defined by Azzam and Dullien (1977) as:

$$
\xi_{p}=\frac{\Delta \bar{P}_{H P}^{*}}{\Delta \bar{P}^{*}}
$$

where $\bar{P}^{*}$ is the dimensionless cross-sectional mean pressure and $\Delta \bar{P}_{H P}^{*}$, the dimensionless pressure loss, is evaluated by applying the Hagen-Poiseuille equation to the pore (the large pipe) and the throat (the small pipe)

$$
\begin{aligned}
\Delta \bar{P}_{H P}^{*} & =\left(\Delta \bar{P}_{H P}^{*}\right)_{p}+\left(\Delta \bar{P}_{H P}^{*}\right)_{t} \\
& =-\frac{32}{R e_{d}} L_{p}^{*}\left(\frac{d}{D}\right)^{4}-\frac{32}{R e_{d}} L_{t}^{*} .
\end{aligned}
$$

Hence, $\xi_{p}$ is a measure of the excess energy dissipation effects which take place in the diverging-converging RUC and which have not been taken into account by the Hagen-Poiseuille equation.

A systematic investigation of the effects of geometric and flow parameters on $\xi_{p}$ has been undertaken. Some typical results for four different values of length ratio $L_{t} / L_{p}$ is illustrated as a function of diameter ratio $D / d$ in Figure 8.15 for $R e_{d}=10$. Because $\Delta \bar{P}^{*}$ is a weak function of $D / d$ (see Subsection 8.2.2), the observed decrease in $\xi_{p}$ as $D / d$ increases is due to a decrease in $\left(\Delta \bar{P}_{H P}^{*}\right)_{p}$. This effect becomes more obvious at small values of $L_{t} / L_{p}$. For the RUC of uniform diameter $(D / d=1)$, the excess momentum loss factor equals to unity. 


\subsection{Behavior of Macroscopic Properties}

Detailed descriptions of the microscopic flow patterns within RUC's have shown the dramatic changes of vortex system for different geometries and Reynolds numbers. The variations of the pore flow fields will certainly alter the interfacial vorticity and pressure distributions. The two integrals (Equations (3.32) and (3.34)) and therefore, the macroscopic parameters $(k, \beta$ and $F o$, etc.) which can be determined numerically, will have different values accordingly.

\subsubsection{Reynolds Number Effect on $k_{v}$ and $F_{0}$}

From their definitions, both $k_{v}$ and $F o$ (Equation (3.38)) are velocity dependent. Further, $k_{v}$ is a function of $d$ and $\xi$. For the purpose of comparison in this subsection, $d=1$ and $\xi=1$ have been applied in the calculations. Figure 8.16 shows the influence of Reynolds number (flow rate) on the Forchheimer number $(F o)$. In the low Reynolds number region $\left(R e_{d}<1\right), F o$ is nearly to zero. It follows that $k_{v}=$ $k$, and a linear relationship between the average pressure gradient and the specific discharge exists (Ref. Equation (3.28)). As the Reynolds number increases, the microscopic inertial effect is no longer negligible. As a dimensionless criterion of the macroscopic nonlinear effect, $F o$ has a significant magnitude. At even higher $R e_{d}$ (e.g., $R e_{d}>6$ ), Fo grows more quickly. A transition zone is presented in the Reynolds number region $\left(3 \leq R e_{d} \leq 10\right)$, where the effect arising from the microscopic inertial force gradually builds up in importance until it dominates in the transport process.

The velocity-dependent permeability $k_{v}$ versus Reynolds number is plotted in Figure 8.17. The symbols in the graphs denote different diameter ratio conditions. 
For the case of $L_{t} / L_{p}=2$ and $L / d=4.875$, the RUC with greater diameter ratio has smaller pressure loss and therefore larger permeability. But this effect becomes less pronounced for higher $R e_{d}$ cases. The geometry effects on $F o$ and $k_{v}$ will be discussed in detail in the following subsections.

\subsubsection{Average Pressure Loss $\Delta \bar{P}^{*}$}

Four cases of average pressure loss $\Delta \bar{P}^{*}$ through the capillary RUC's are given in Figure 8.18, for $R e_{d}=1,10,20$ and 80 , taking the diameter ratio and the length ratio as the independent parameters. One may see from the graphs that the pressure loss is very much dependent on the length ratio (the ratio of throat length to pore length), but is weakly dependent on the diameter ratio. This result supplements the research by Dullien and Azzam (1973) and Azzam and Dullien (1977). In their work, the importance of the length ratio was not taken into account in either the theoretical development or the experimental results.

In Figure 8.18, the dashed lines represent the pressure drop for $D / d=1$, namely, a straight pipe with diameter $d$. For the case of $R e_{d}=1$ (Figure 8.18(A)), the pressure losses for the diverging-converging RUC are all smaller than those for the straight pipe. The explanation for this phenomenon is that the viscous effect is the main source of the pressure loss at low $R e_{d}$ values and the viscous loss in the pore segment (large pipe) is always reduced when the diameter ratio increases. However, this situation changes at higher Reynolds numbers (Figures 8.18(C) and (D)). Here the pressure loss relating to the inertial effect grows and exceeds the pressure gains by the pore effect. Therefore, in some cases (large length ratio), the average pressure losses are larger than those for the straight pipe. 


\subsubsection{Interfacial Drag Force}

With known microscopic flow fields, the interfacial viscous and pressure integrals of Equations (3.26) and (3.27) can be numerically obtained for different RUC's. The influence of the diameter ratio and the length ratio on the dimensionless integrals $V_{I N T}^{*}$ and $P_{I N T}^{*}$ as a function of the Reynolds number were studied for different $L / d$ patterns. Some typical results are presented in Figure 8.19 for $V_{I N T}^{*}$ and Figure 8.20 for $P_{I N T}^{*}$. For the given length to diameter ratio, it can be seen in Figure 8.19 that the viscous integral in all cases increases as the diameter ratio $(D / d)$ decreases (Ref. Figure 8.1(A)), and converges to the asymptotic value at $D / d=1$, where Hagen-Poiseuille flow results. The effects of the diameter ratio change become less important at large $D / d$ values since it is evident from Figure 8.19 that $V_{I N T}^{*}$ has gradually achieved an asymptotic value. The explanation for this phenomenon is that the surface viscous stress in the pore is reduced when the diameter ratio increases. The increases of the length ratio (Ref. Figure 8.1(B)), $L_{t} / L_{p}$, result in a longer throat and therefore, a larger surface viscous force. The pressure integral, $P_{I N T}^{*}$, represents the resistant force resulting from the geometric formation of the flow channels. For a straight pipe $(D / d=1)$, this force equals to zero. As shown in Figure 8.20, the computational results predicted a maximum at the diameter ratio $D / d \simeq 1.5 \sim 2$. As the pore Reynolds number increases, the diameter ratio corresponding to the maximum pressure integral decreases.

From their definitions, both pressure and viscous integrals are dependent on the local flow mechanisms. Figure 8.21 shows the influence of the pore Reynolds number on the total interfacial drag force, i. e., $V_{I N T}^{*}+P_{I N T}^{*}$. The dashed lines in the graph represent the linear relations between the interfacial drag force and the Reynolds number. It is evident that at certain values of Reynolds number, 
depending on the microstructure, the interfacial drag force gradually deviates from the linear relation due to the microscopic inertial effects. This result suggests that the closure representation of this force should include higher order terms with respect to the averaged velocity for the cases of high $\mathrm{F}$-number flow.

The effects of the length ratio, $L_{t} / L_{p}$, on the total drag force may be observed from Figure 8.22 for two Reynolds numbers. The asymptote at $L_{t} / L_{p} \rightarrow \infty$ is given by the dashed line. It can be seen that the drag force increases as the length ratio becomes larger.

The macroscopic parameters describing the porous structure, such as porosity and areosity, may be calculated for each RUC of the present model. An attempt has been made in this research to correlate the interfacial drag force with these parameters. However, it was found that such correlations are hard to achieve. To be more specific, RUC's with equal values of porosity and areosity at the same flow rate, may possess very different values of the interfacial drag force depending on the pore configuration. These results suggest that constitutive correlations should be more carefully formulated in order to simulate the dependence of the integrals on the local flow condition and geometrical configuration.

\subsubsection{Geometric Effects on $k_{v}, F_{o}$ and $\beta$}

As mentioned above, the values of $k_{v}$ and $\beta$ are dependent on a characteristic length and the areosity. In this study, the diameter of the throat is conveniently taken as the characteristic length, and $d=1$ throughout the calculations. But for the present model, there is no obvious cross-sectional area $A_{b}$ to be used in defining the aerosity. For the sake of comparison of the results for different geometries, a cross section $A_{b}^{*}=4.5 * 4.5$ has been chosen (Figure 4.1 ). Numerically, this area 
equals the quotient of the total cross-sectional area of the model porous medium to the number of parallel flow channels embedded in the impervious solid. Following the definition of $A_{b}^{*}$, the aerosity and the porosity of the unit cell may be calculated for each case.

The influence of diameter ratio and length ratio on $F o$ and $k_{v}$ as a function of Reynolds number were studied for different $L / d$ patterns. Two typical results are presented in Figure 8.23 for $k_{v}$ and Figure 8.24 for $F o$. It may be noted that the length ratio effect on $k_{v}$ (or $F_{o}$ ) for the Reynolds numbers studied becomes less important at large $L_{t} / L_{p}$ values. The magnitude of the Darcy's law permeability $k$ can be found approximately from the curve for $R e_{d}=1$ in Figure 8.23. The results suggest that for a porous medium with large throat-to-pore length ratio $\left(L_{t} / L_{p}>\right.$ $3)$ and large diameter ratio $(D / d>2.5)$, both the Darcy's law permeability $k$ and the velocity dependent permeability $k_{v}$ are approximate constants which depend on Reynolds number and the length/diameter ratio only. Similar to the analysis of the pressure loss $\Delta \bar{P}^{*}, k_{v}$ and $F o$ are weak functions of the diameter ratio. This conclusion can be obtained by comparing $k_{v}$ graphs for $D / d=4$ (Figure 8.23) and $D / d=2$ (Figure 8.25).

Figure 8.26 shows the permeability $k_{v}$ at different length/diameter ratios. For a fixed length and a fixed diameter ratio, the larger the value of $L / d$, the less frequent will be the change in geometry (Ref. Figure $8.1(\mathrm{C})$ ). This results in smaller pressure losses, and therefore in greater permeability.

Another typical result for the relationship between $k_{v}$ and the porosity is given in Figure 8.27. It can be seen that $k_{v}$ increases quickly as the porosity becomes larger for the case presented. The reason for this is that for fixed diameter ratio and length/diameter ratio, the change of the porosity results from the change of 
the length ratio, and a larger value of the porosity corresponds to a smaller length ratio and a smaller pressure loss, which results in a larger permeability. It should be mentioned that this correlation is performed with the diameter ratio and the length to diameter ratio kept unchanged.

The geometric effects on the inertia coefficient $\beta$ are given in Table 8.1 by taking $A_{b}^{*}=4.5 * 4.5$ as the reference area. In large diameter ratio and small length ratio cases, the variation of the cross-sectional area along the axis is more pronounced and abrupt, which results in more visible change of the velocity, and therefore, in high inertial effects. It was found in the computations that the inertia coefficient $\beta$ depends weakly on the velocity. This may hint that a higher order approximation is needed for the present geometric model.

\begin{tabular}{|c|c|c|c|c|}
\hline \multicolumn{5}{|c|}{ Table 8.1 Geometric effects on the inertial coefficient $\beta$ for $L / d=4.875$. } \\
\hline$L_{t} / L_{p}$ & 1.143 & 2 & 3.167 & 4 \\
\hline 1.5 & 33.704 & 31.842 & 29.449 & 25.926 \\
\hline 2 & 46.600 & 43.476 & 33.903 & 29.050 \\
\hline 3 & 48.395 & 46.002 & 35.565 & 29.112 \\
\hline 4 & 49.858 & 46.401 & 37.360 & 30.446 \\
\hline
\end{tabular}

\subsection{Physical Explanation of Nonlinear Effect in High F-Number Flow}

The analysis of the flow phenomena at the pore level indicates that the particles of fluid move tortuously through the channel. The inertial term (Ref. Equation (3.12)), which is proportional to the fluid density and its acceleration, is a nonzero quantity at every point inside the capillary because of the sudden geometry changes. This term can be regarded as a force term. Dynamically, the terms in the microscopic momentum equation (i.e., inertial, pressure, viscous and body 
force) should be in balance at every point of the fluid phase. However, the different terms, which represent different physical phenomena, play changing rôles in different flow conditions. The data for the mean values of these microscopic forces and the relative magnitude of the macroscopic parameters (Fo or $k_{v}$ ) at different Reynolds numbers will be helpful for precisely describing the nonlinear phenomena for high F-number flow. Accordingly, Equation (3.12) has been integrated over every cross-section for various Reynolds numbers. The mean values of inertial, pressure and viscous forces through the capillary are illustrated in Figure 8.28. For a convenient presentation of the results, the curves are normalized with the value of the pressure term at the entrance. At a low value of Reynolds number (Figure $8.28(\mathrm{~A})$ ), the inertial force is negligibly small everywhere. Here the pressure force is basically balanced by the viscous force. For the higher value of $R e_{d}$ at which the macroscopic nonlinear behavior becomes more and more observable (Ref. Figure 8.16), the inertial force becomes nonnegligible as compared with the viscous force in the region of the sudden geometry change (Figure 8.28(B)). At even higher Reynolds number cases (Figure 8.28(C)), the inertial force and viscous force contribute equally in balancing the pressure change. It may be noticed in Figures $8.28(\mathrm{~A}) \sim(\mathrm{C})$ that the inertial effect is concentrated in the vicinity of the two convex corners for the present model. The numerical residuals (a measure of numerical error) of the integrations are also plotted in Figure 8.28. It can be seen that they are smaller quantities except near the corners at the highest Reynolds number. At high Reynolds number, the residuals increase, but are still small relative to the dominant terms.

As already mentioned in Subsection 3.2.1, the microscopic inertial term in the averaged momentum equation (3.18) disappears due to the no-slip condition on the fluid/solid interface. But it is important to remember that its effect has been 
stored in the distorted velocity and pressure fields and in turn is manifested in the interfacial integrals of the pressure and the viscous force.

From the discussions above, it seems reasonable to conclude that if the growth of interfacial drag forces at high flow velocity is taken as the superficial reason for the nonlinear behavior of the flow (Hassanizadeh and Gray, 1987), the fundamental source of the phenomena should at last be attributed to the microscopic inertial force.

\subsection{Remarks}

The numerical solutions for fluid flow in a porous medium modelled by periodic diverging-converging capillaries have been performed for the purpose of quantitatively analyzing the relationship between the microscopic flow and the macroscopic responses. The result analyses presented in this chapter support the following conclusions.

1. The development of the fluid motion at different geometries and Reynolds numbers reveals diverse flow patterns as distinguish from simple expansion or contraction flow;

2. The numerical analysis of microscopic flow and the macroscopic response clearly shows that the fundamental reason for the onset of nonlinear flow $\left(R e_{d} \approx 10\right)$ is the microscopic inertial force which has been manifested in the interfacial drag force;

3. The interfacial drag force depends strongly on the geometric and flow conditions at the microscopic level. The four parameters characterizing the microscopic flow for the present model are the diameter ratio, the length ratio, the 
length to diameter ratio and the pore Reynolds number, although not all of them are of the same importance in all circumstances;

4. The permeability $k$ (or $k_{v}$ ) and the inertial coefficient $\beta$ are complex functions of the microstructure. For the periodic diverging-converging capillary model, the microstructure is characterized by three dimensionless parameters, namely, the diameter ratio, the length/diameter ratio and the length ratio. Compared with the length/diameter ratio and the length ratio, $k_{v}$ (or $k$ ) and $\beta\left(\right.$ or $F_{0}$ ) are weakly dependent on the diameter ratio for $D / d>2.5$. The velocity dependent permeability $k_{v}$ (or the Darcy's law permeability $k$ ) may be approximately treated as a constant which depends on the Reynolds number and the length/diameter ratio when $L_{t} / L_{p}>3$ and $D / d>2.5$. 

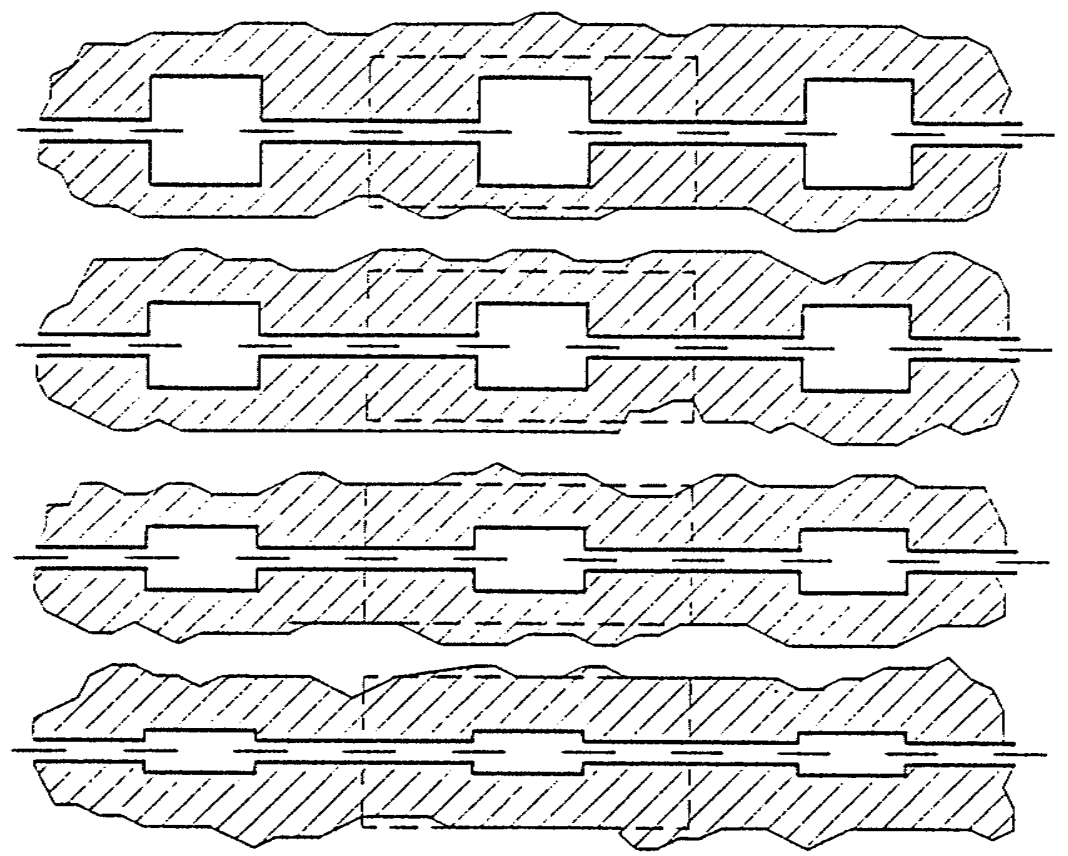

(A). RUCs with diameter ratio $(D / d)$ changes only.

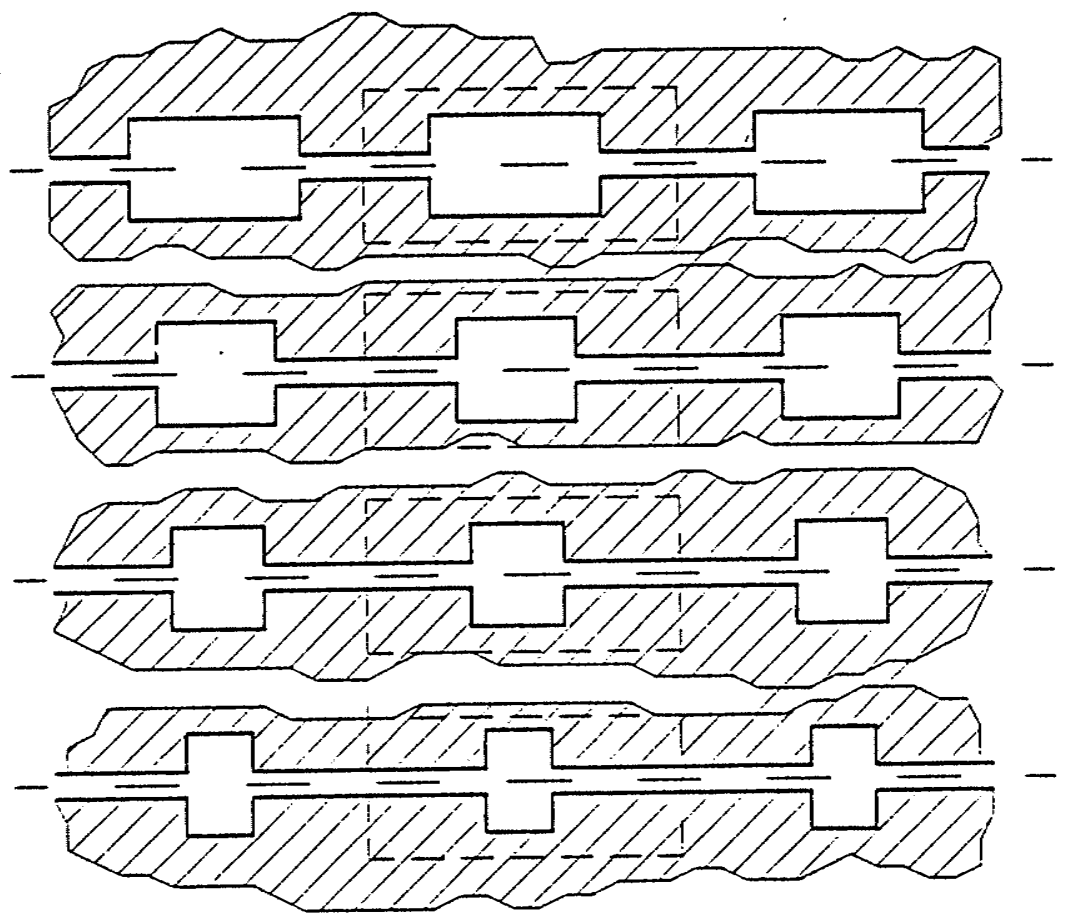

(B). RUCs with length ratio $\left(L_{t} / L_{p}\right)$ changes only.

Fig. 8.1. Geometric configurations of the RUCs. 


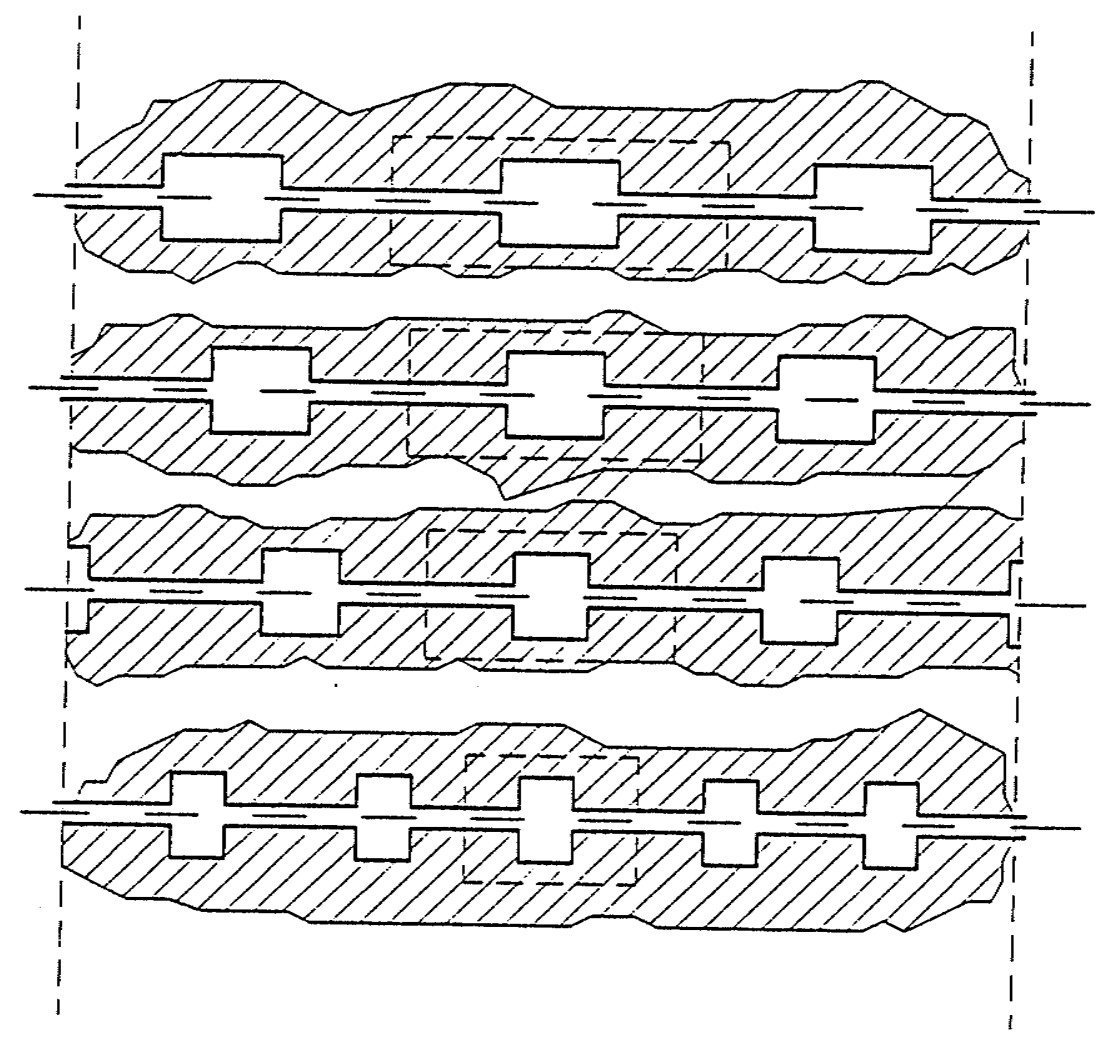

(C). RUCs with length to diameter ratio $(L / d)$ changes only.

Fig. 8.1. Geometric configurations of the RUCs. 


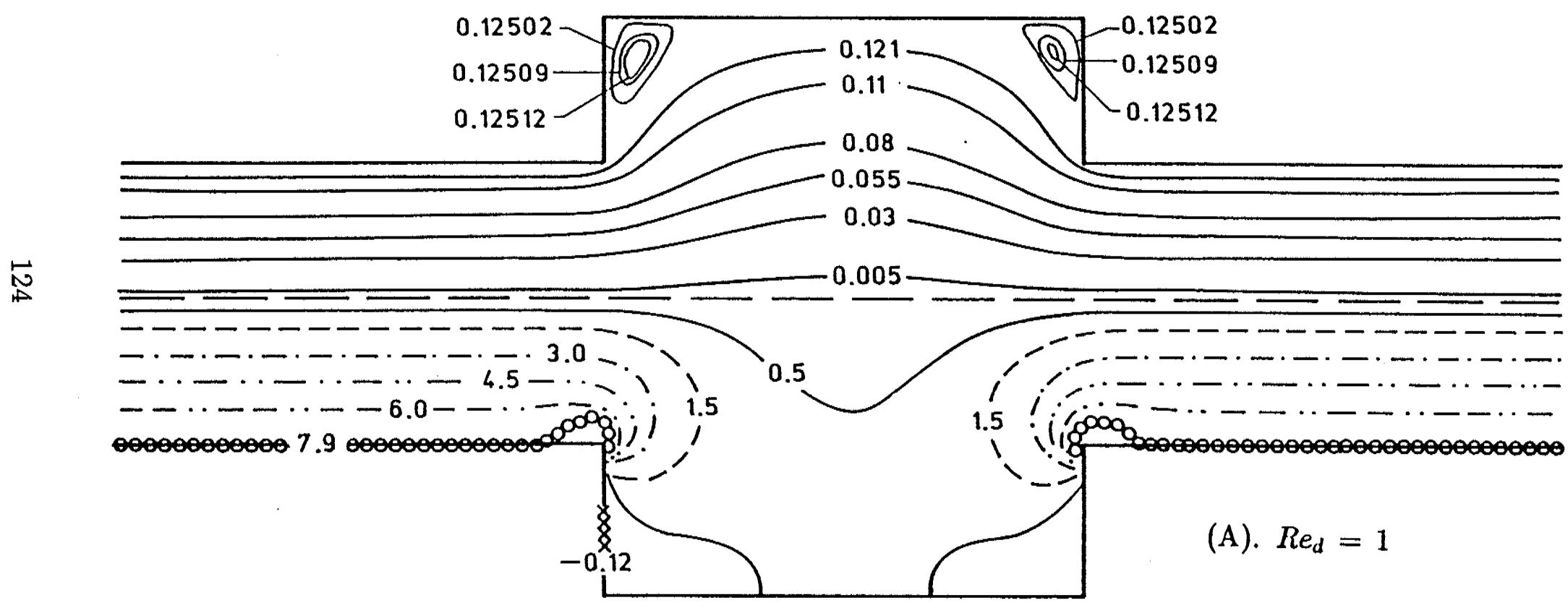

Fig. 8.2. Streamlines and vorticity contours for the RUC of $D / d=2, L_{t} / L_{p}=2$, and $L / d=4.875$. 


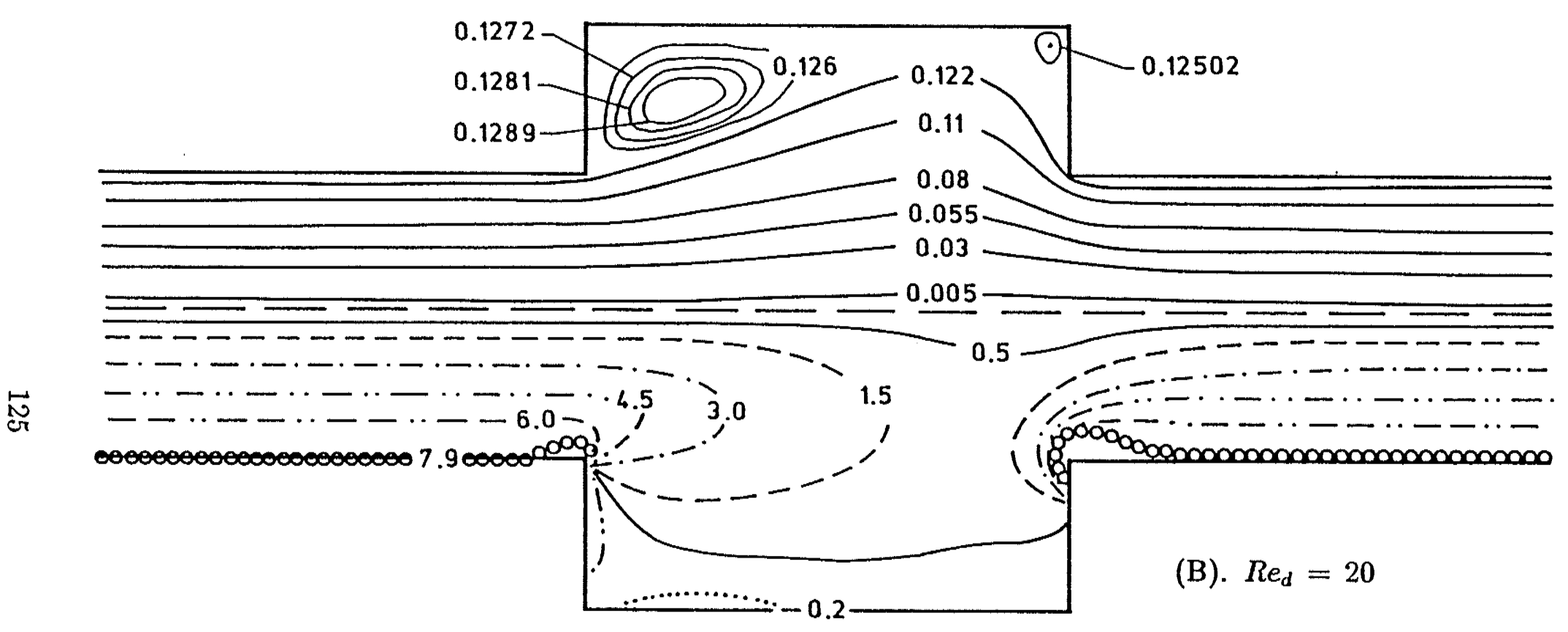

Fig. 8.2. Streamlines and vorticity contours for the RUC of $D / d=2, L_{t} / L_{p}=2$, and $L / d=4.875$. 


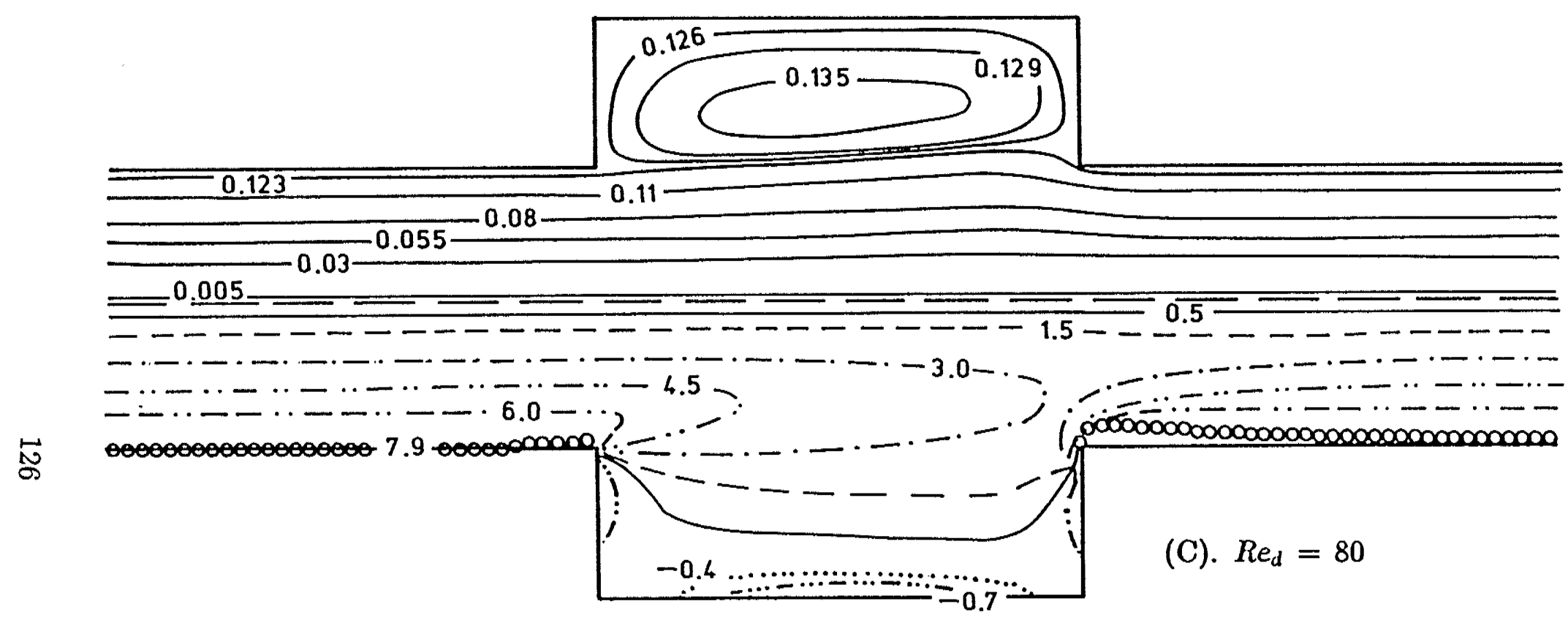

Fig. 8.2. Streamlines and vorticity contours for the RUC of $D / d=2, L_{t} / L_{p}=2$, and $L / d=4.875$. 


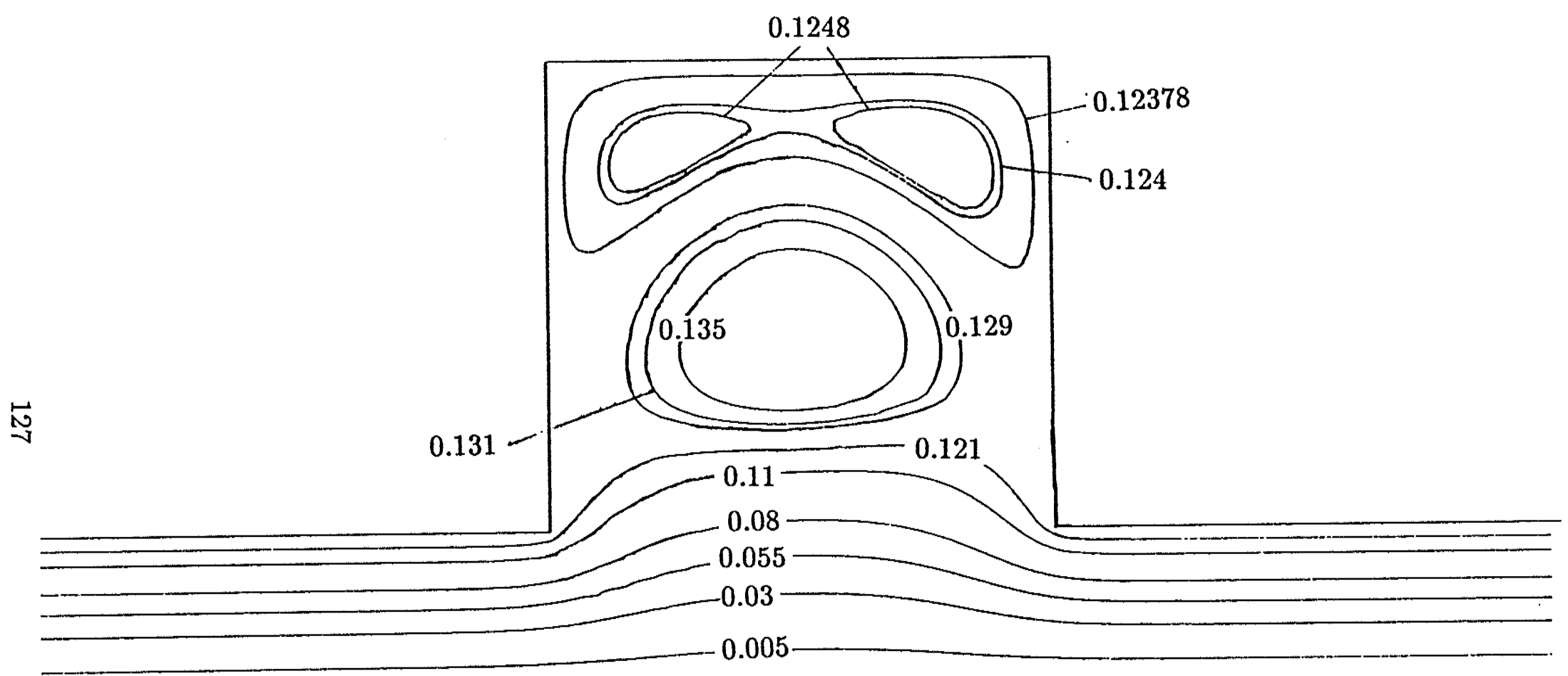

(A). $R e_{d}=0.01$

Fig. 8.3. Flow patterns for the RUC of $D / d=4, L_{t} / L_{p}=2$, and $L / d=4.875$. 


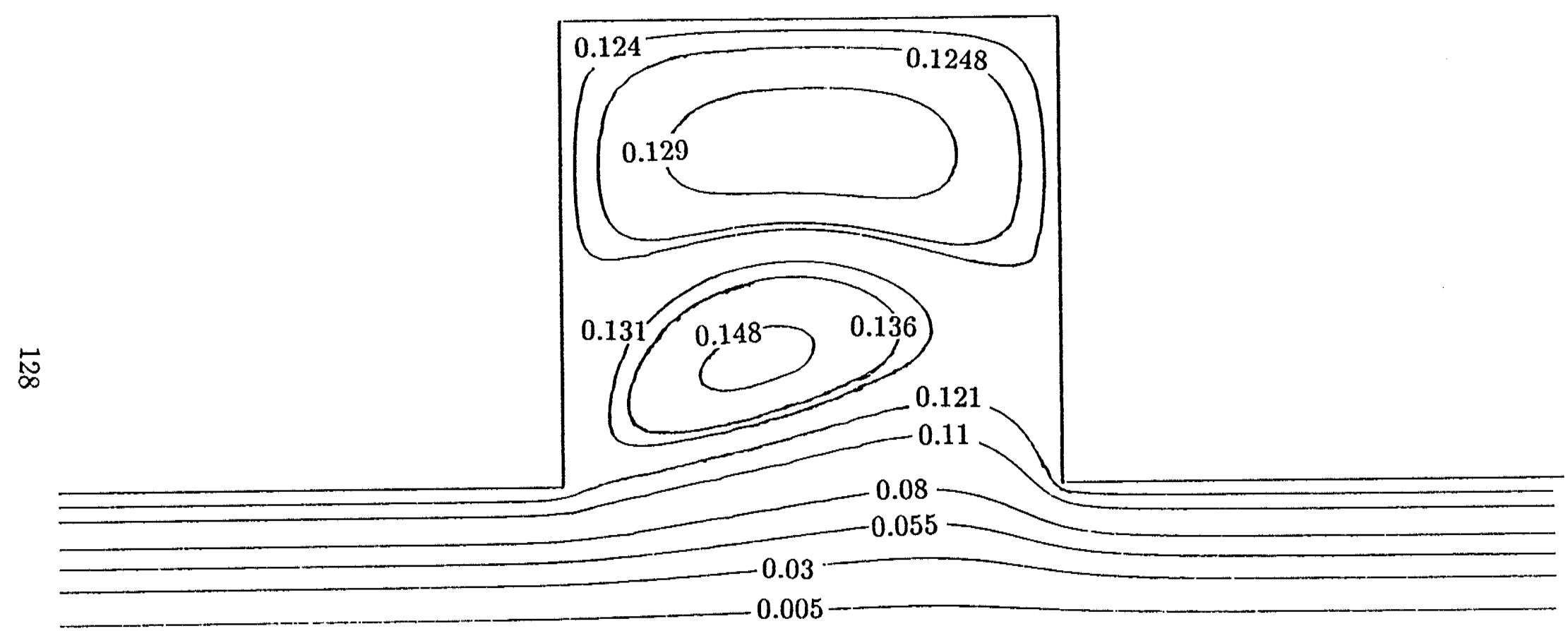

$$
\text { (B). } R e_{d}=20
$$

Fig. 8.3. Flow patterns for the RUC of $D / d=4, L_{t} / L_{p}=2$, and $L / d=4.875$. 


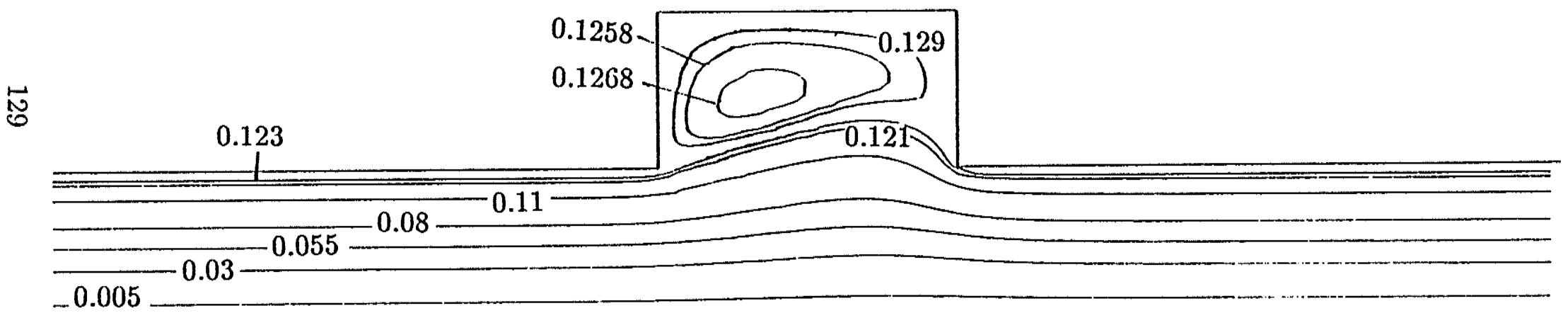

Fig. 8.4. Flow patterns for the RUC of $D / d=2, L_{t} / L_{p}=4$, $L / d=4.875$ and $R e_{d}=20$. 


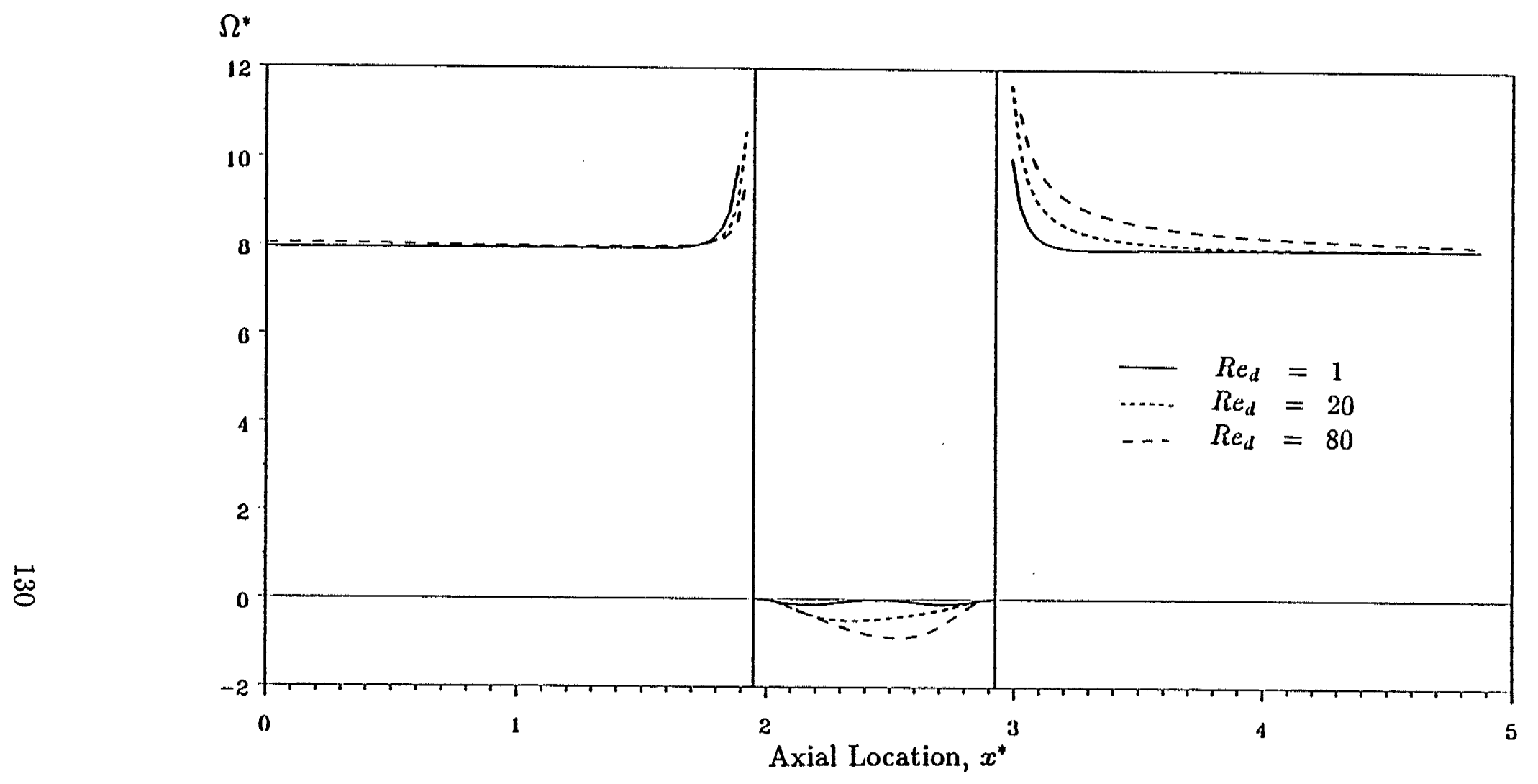

(A). $L_{t} / L_{p}=4$

Fig. 8.5. Distributions of interfacial vorticity for the RUC of $D / d=2$ and $L / d=4.875$. 


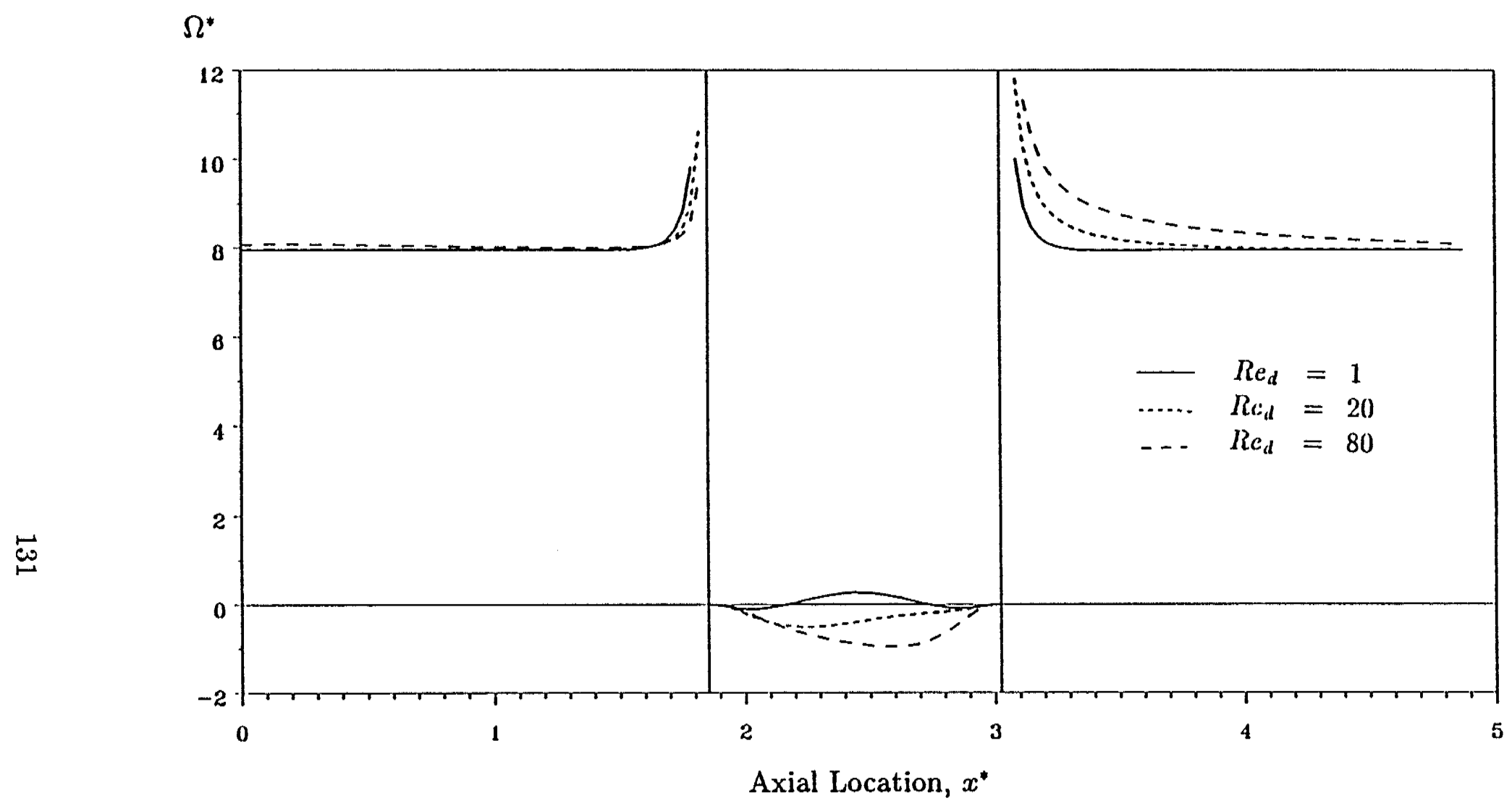

(B). $L_{t} / L_{p}=3.167$

Fig. 8.5. Distributions of interfacial vorticity for the RUC of $D / d=2$ and $L / d=4.875$. 


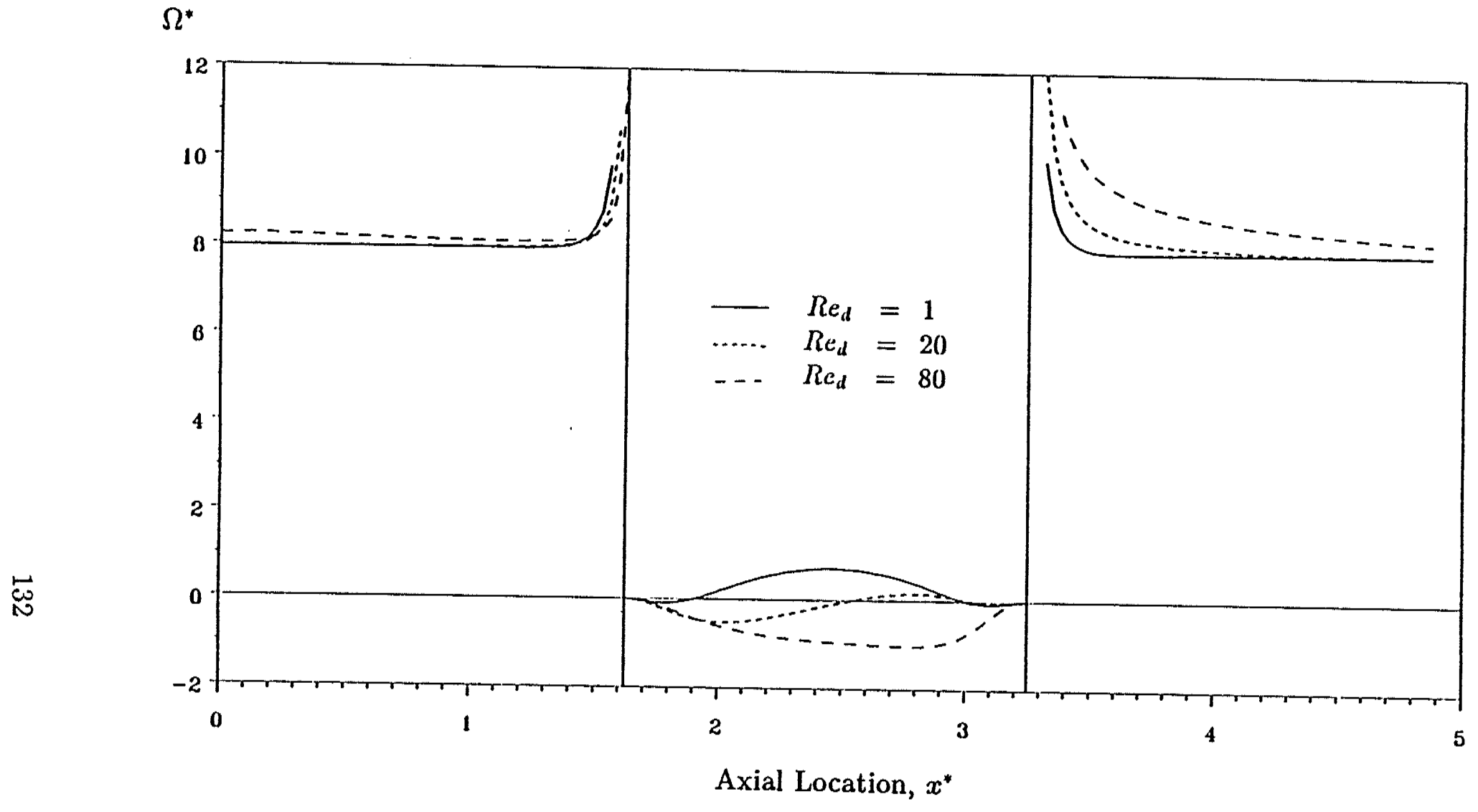

(C). $L_{t} / L_{p}=2$

Fig. 8.5. Distributions of interfacial vorticity for the RUC of $D / d=2$ and $L / d=4.875$. 


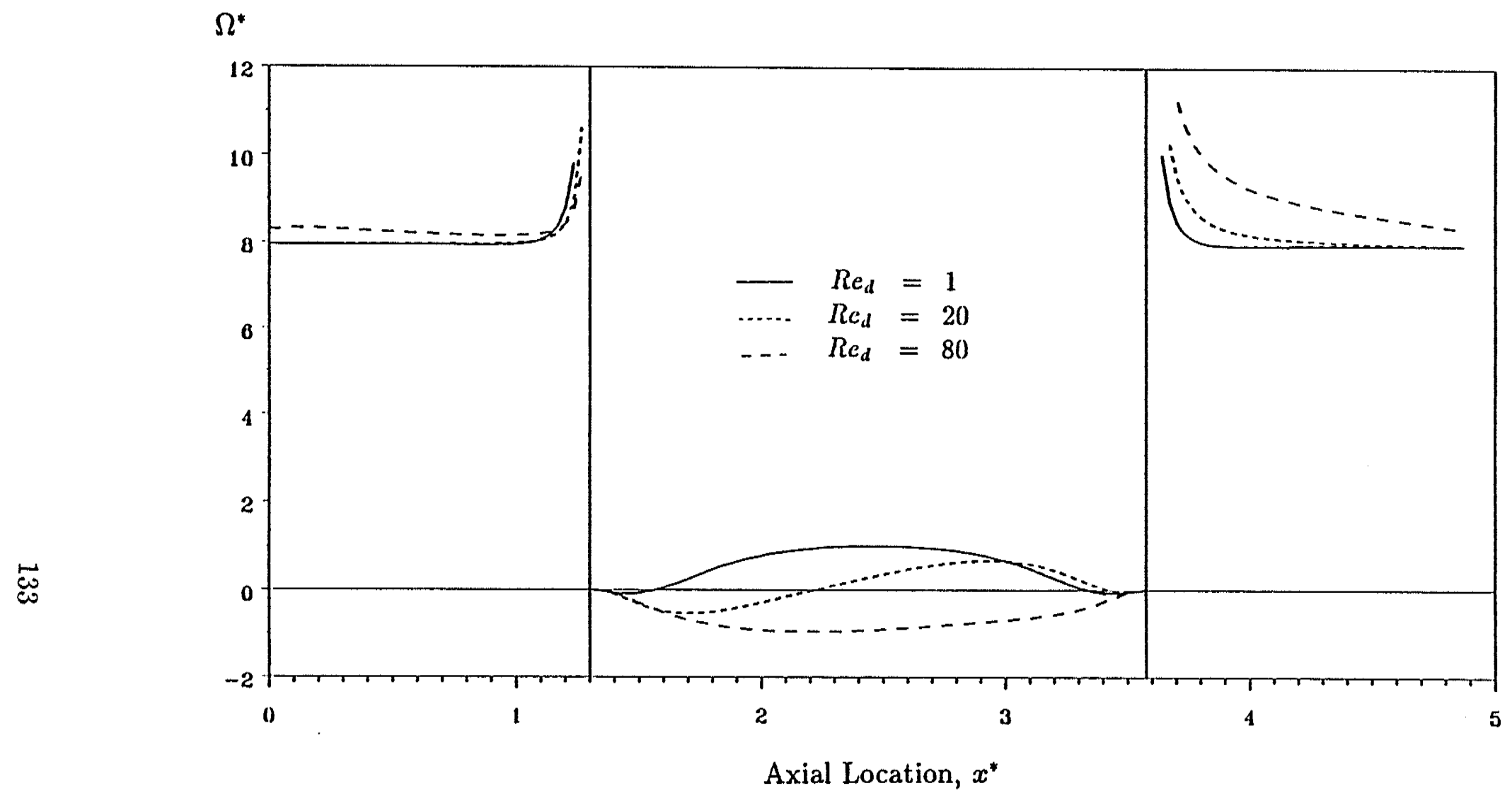

(D). $L_{t} / L_{p}=1.143$

Fig. 8.5. Distributions of interfacial vorticity for the RUC of $D / d=2$ and $L / d=4.875$. 


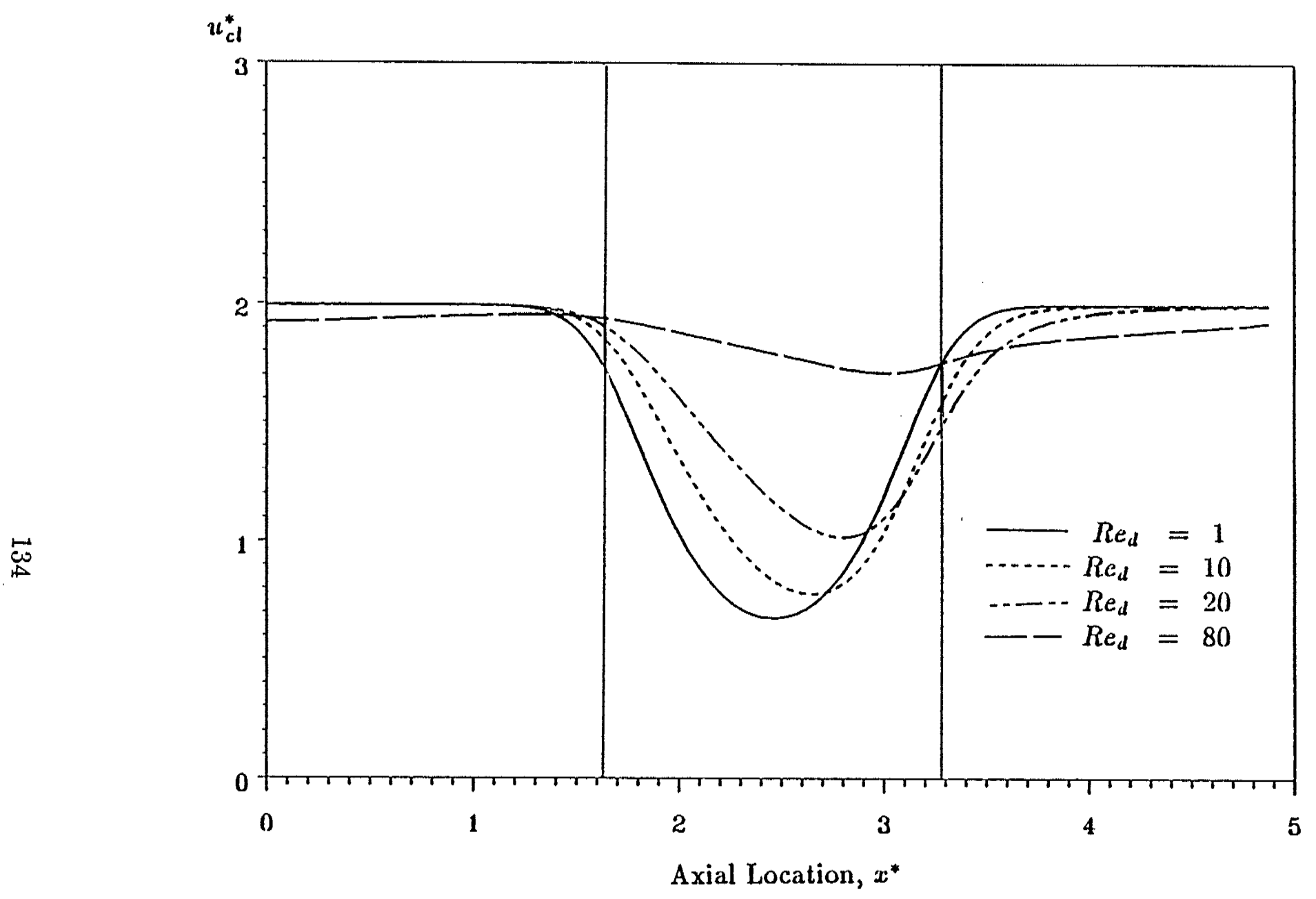

(A). $L_{t} / L_{p}=2$

Fig. 8.6. Centerline velocity distributions for the RUC of $D / d=2$ and $L / d=4.875$. 


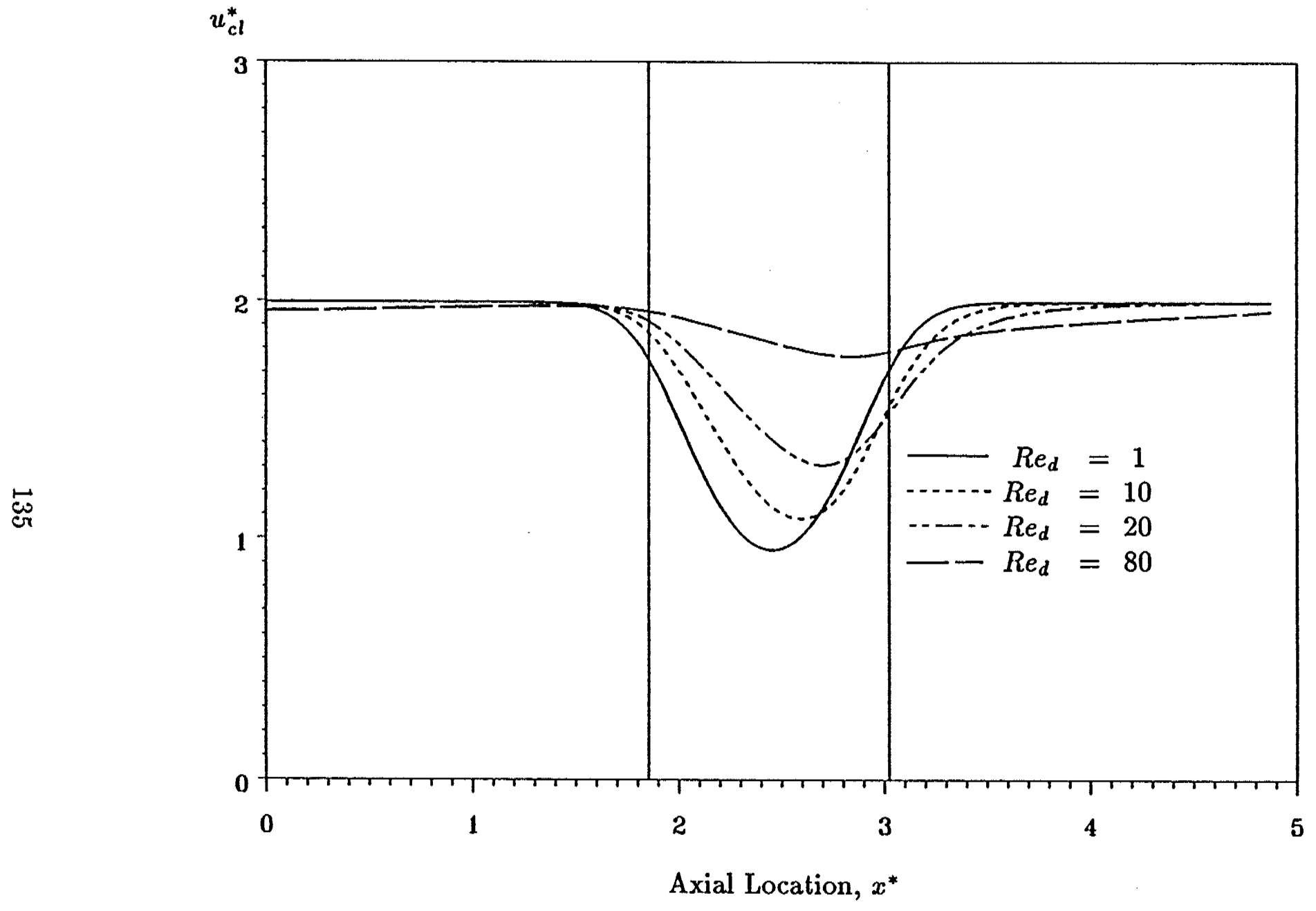

(B). $L_{t} / L_{p}=3.167$

Fig. 8.6. Centerline velocity distributions for the RUC of $D / d=2$ and $L / d=4.875$. 


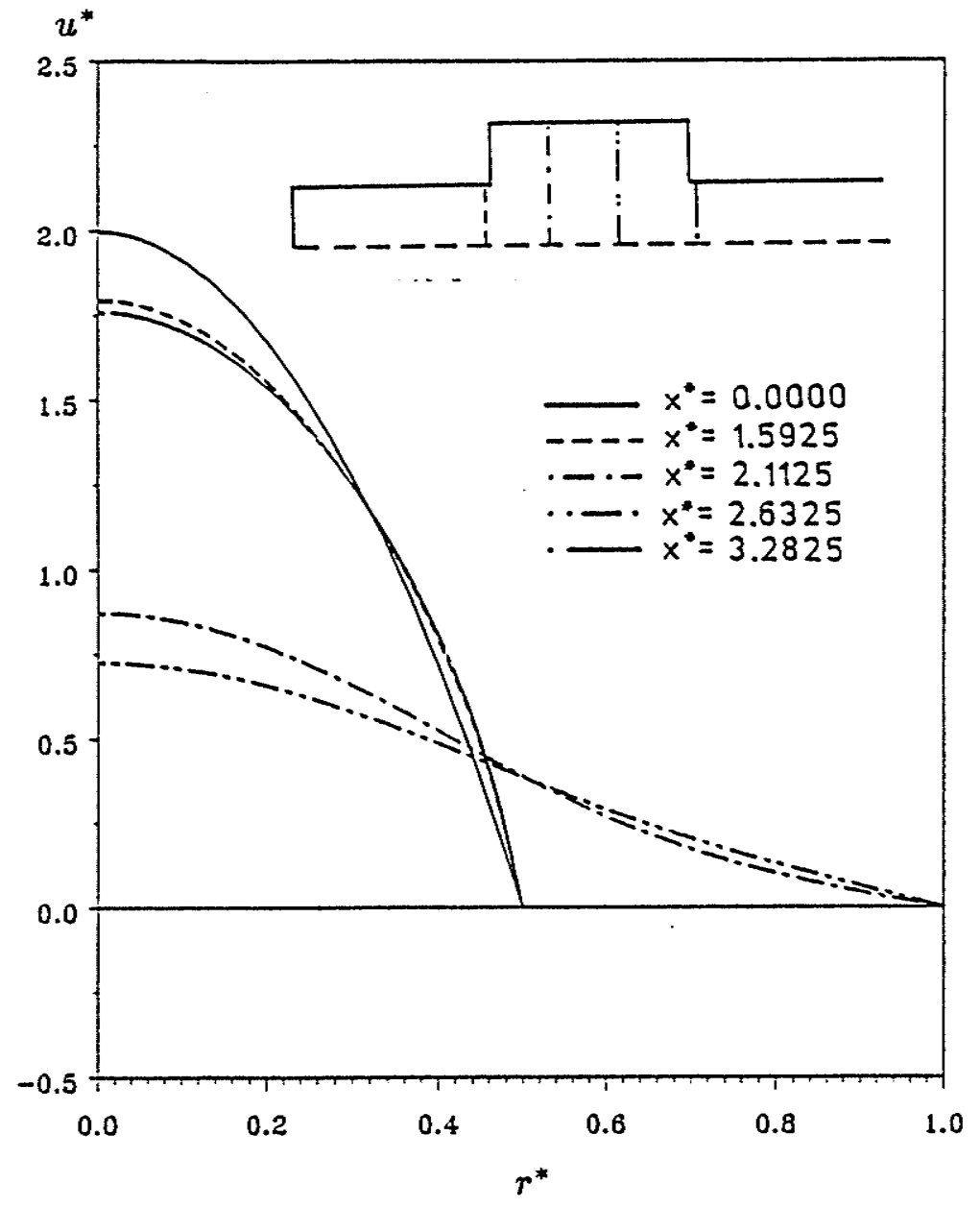

(A). Axial velocity profiles

Fig. 8.7. Velocity profiles at representative axial positions for the RUC of $D / d=2, L_{t} / L_{p}=2, L / d=4.875$ and $R e_{d}=1$. 


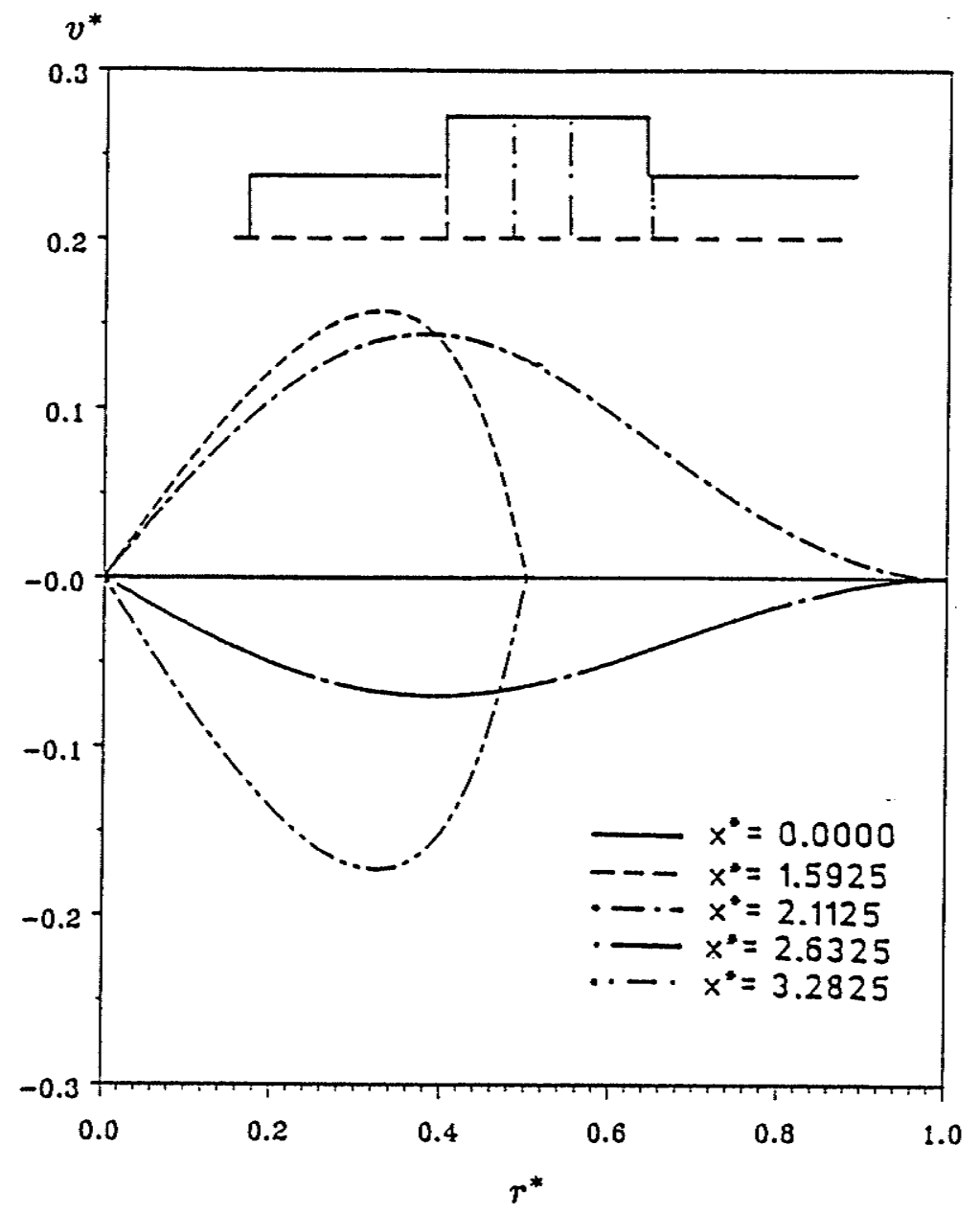

(B) Radial velocity profiles

Fig. 8.7. Velocity profiles at representative axial positions for the RUC of $D / d=2, L_{t} / L_{p}=2, L / d=4.875$ and $R e_{d}=1$. 


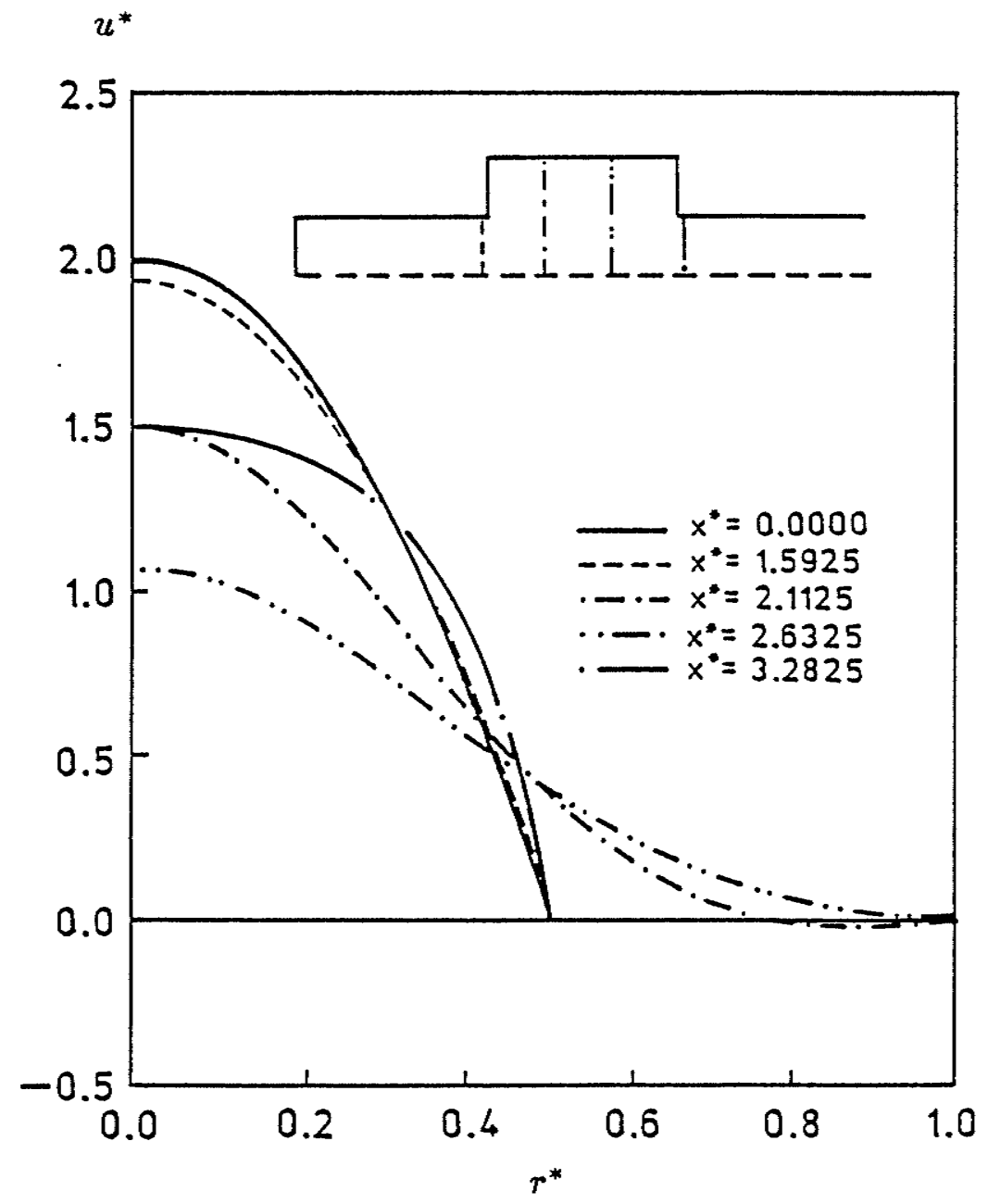

(A). Axial velocity profiles

Fig. 8.8. Velocity profiles at representative axial positions for thi of $D / d=2, L_{t} / L_{p}=2, L / d=4.875$ and $R e_{d}=20$. 


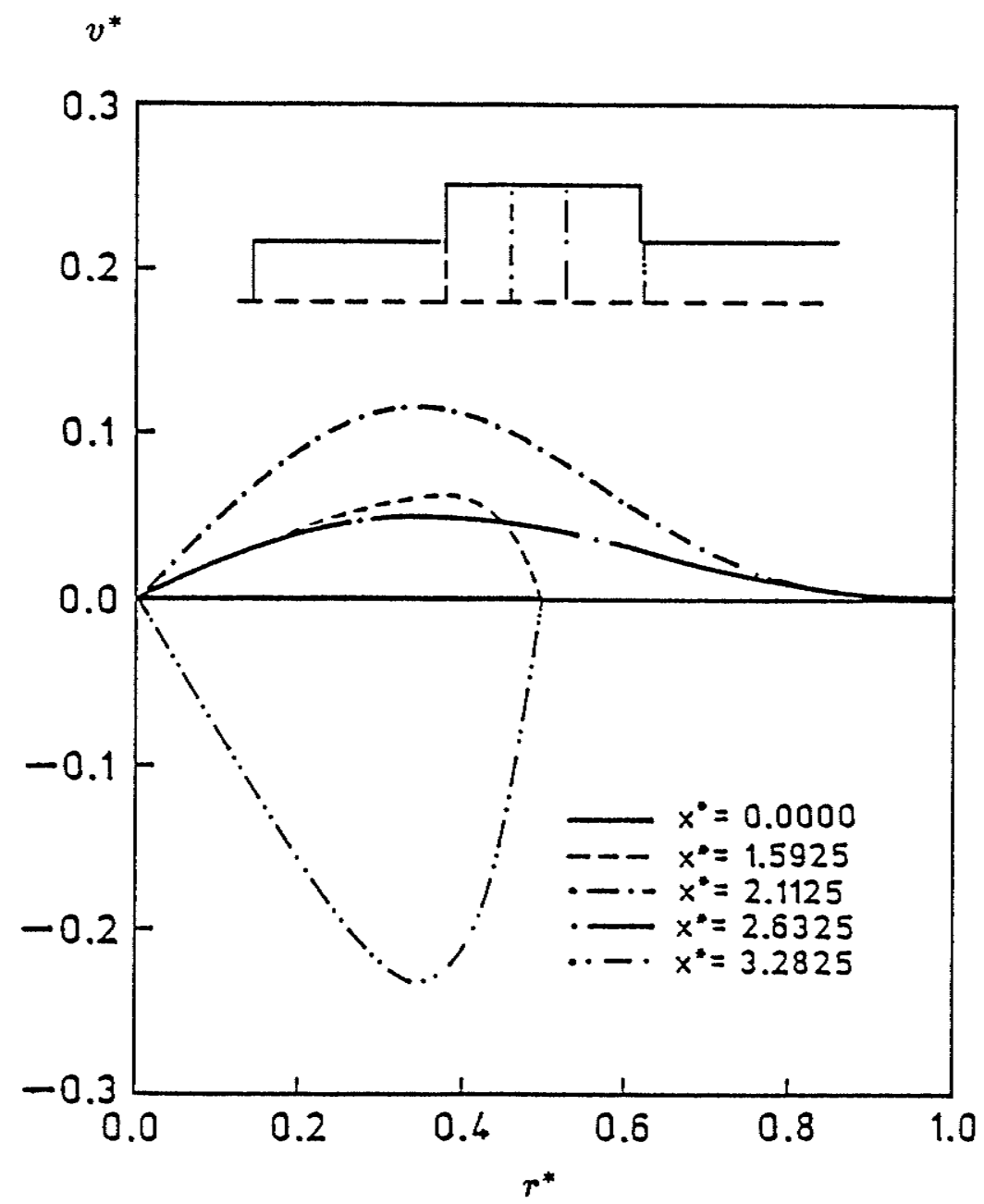

(B) Radial velocity profiles

Fig. 8.8. Velocity profiles at representative axial positions for the RUC of $D / d=2, L_{t} / L_{p}=2, L / d=4.875$ and $R e_{d}=20$. 


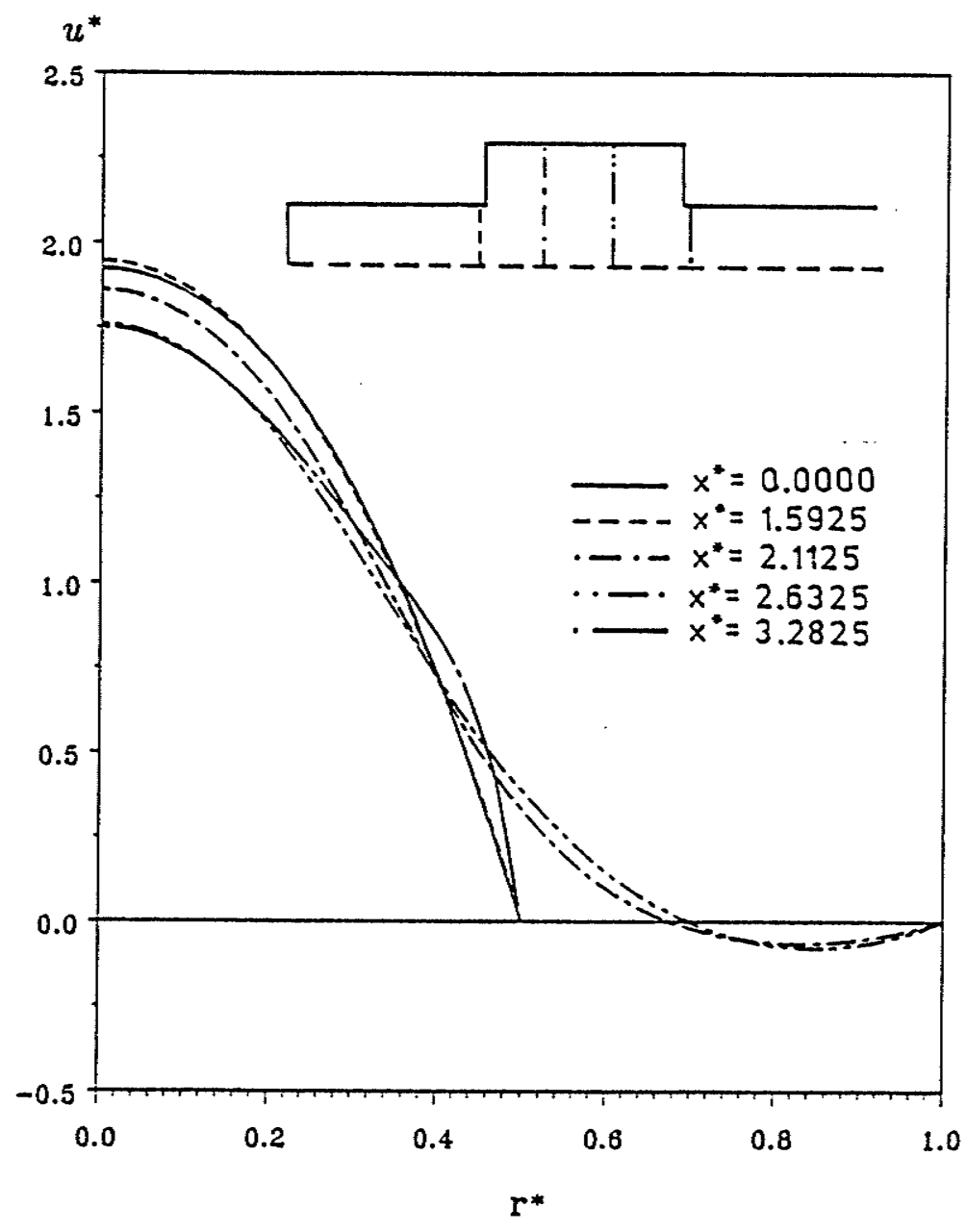

(A). Axial velocity profiles

Fig. 8.9. Velocity profiles at representative axial positions for the RUC of $D / d=2, L_{t} / L_{p}=2, L / d=4.875$ and $R e_{d}=80$. 


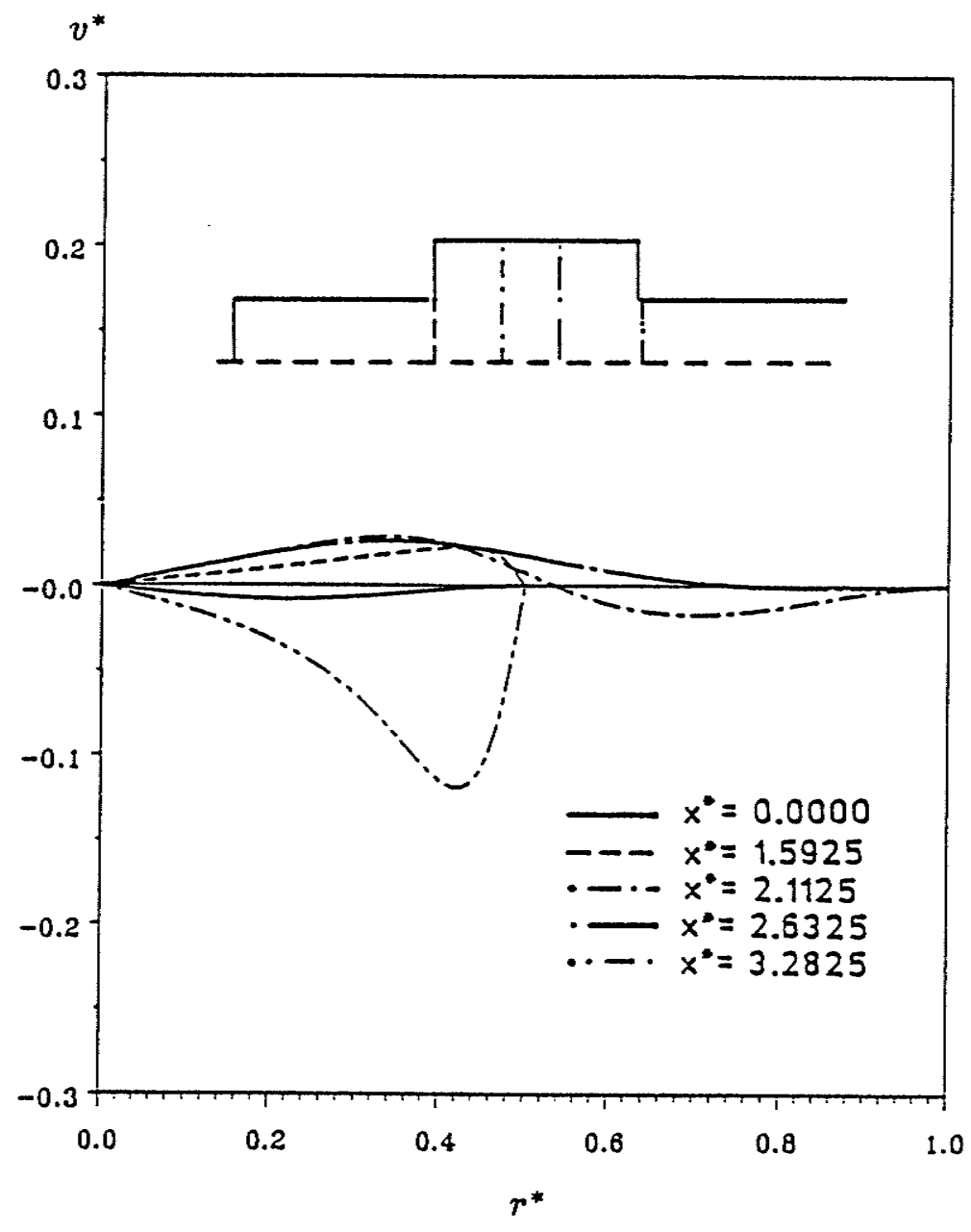

(B) Radial velocity profiles

Fig. 8.9. Velocity profiles at representative axial positions for the RUC of $D / d=2, L_{t} / L_{p}=2, L / d=4.875$ and $R e_{d}=80$. 


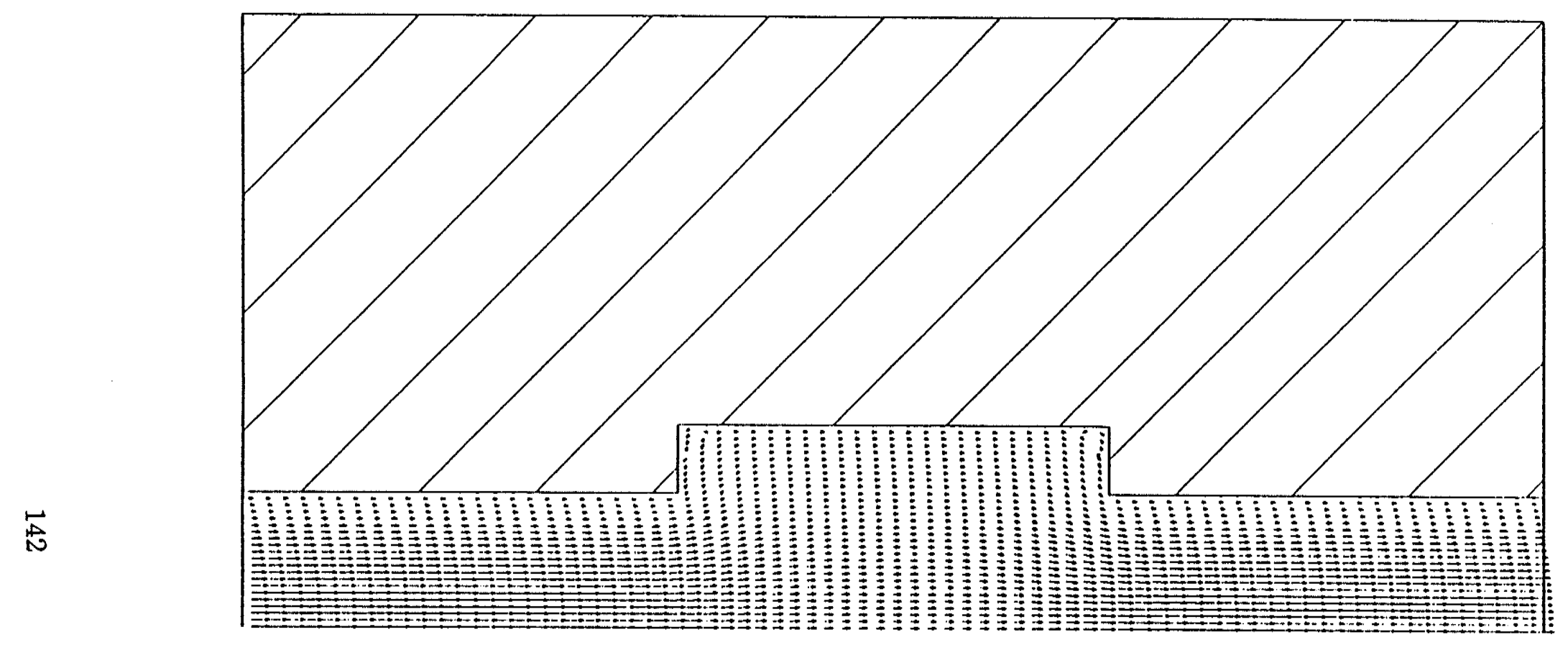

(A) $\cdot R e_{d}=1$.

Fig. 8.10. Velocity field for the RUC of $D / d=1.5, L_{t} / L_{p}=2$ and $L / d=4.875$. 


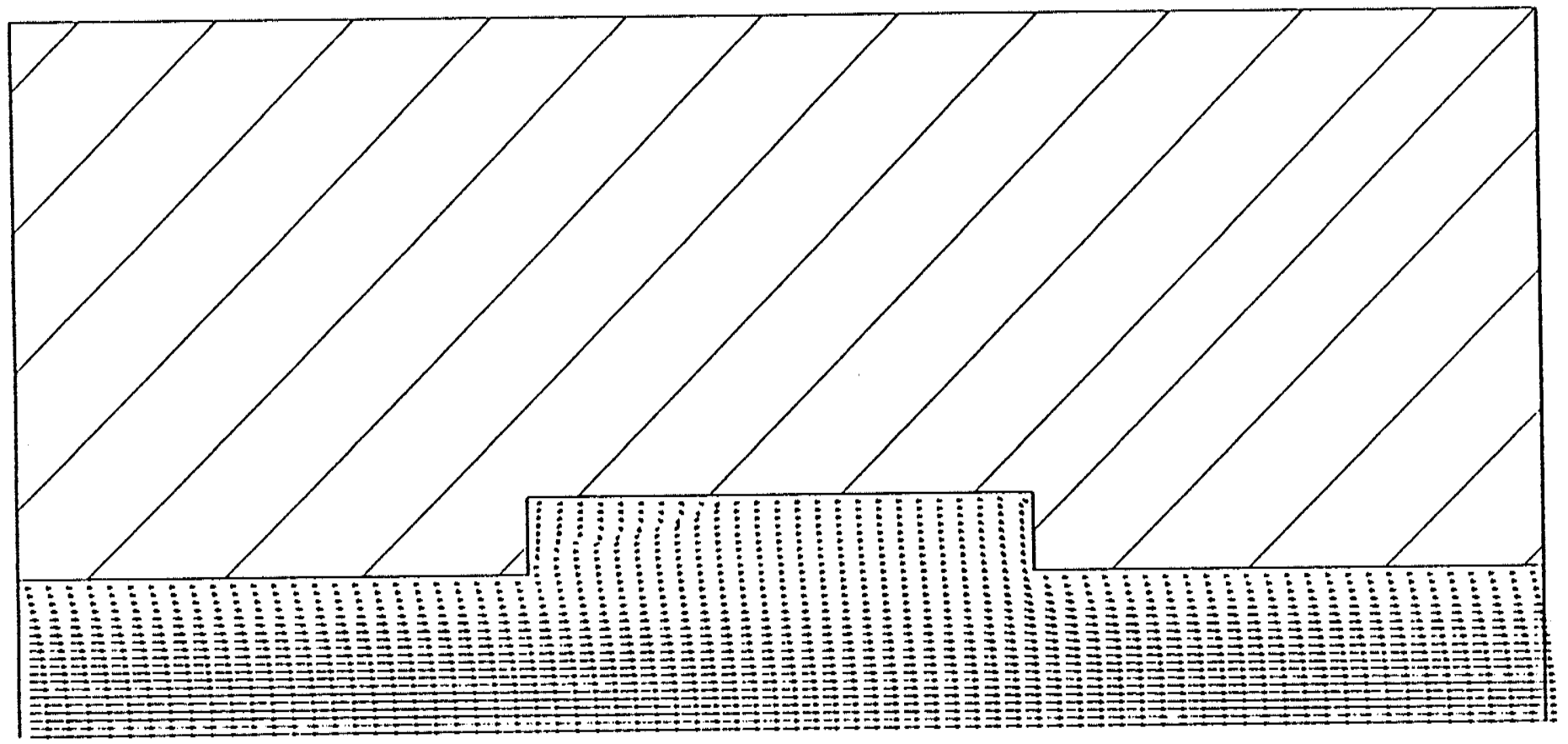

(B) $\cdot R e_{d}=50$

Fig. 8.10. Velocity field for the RUC of $D / d=1.5, L_{t} / L_{p}=2$ and $L / d=4.875$. 


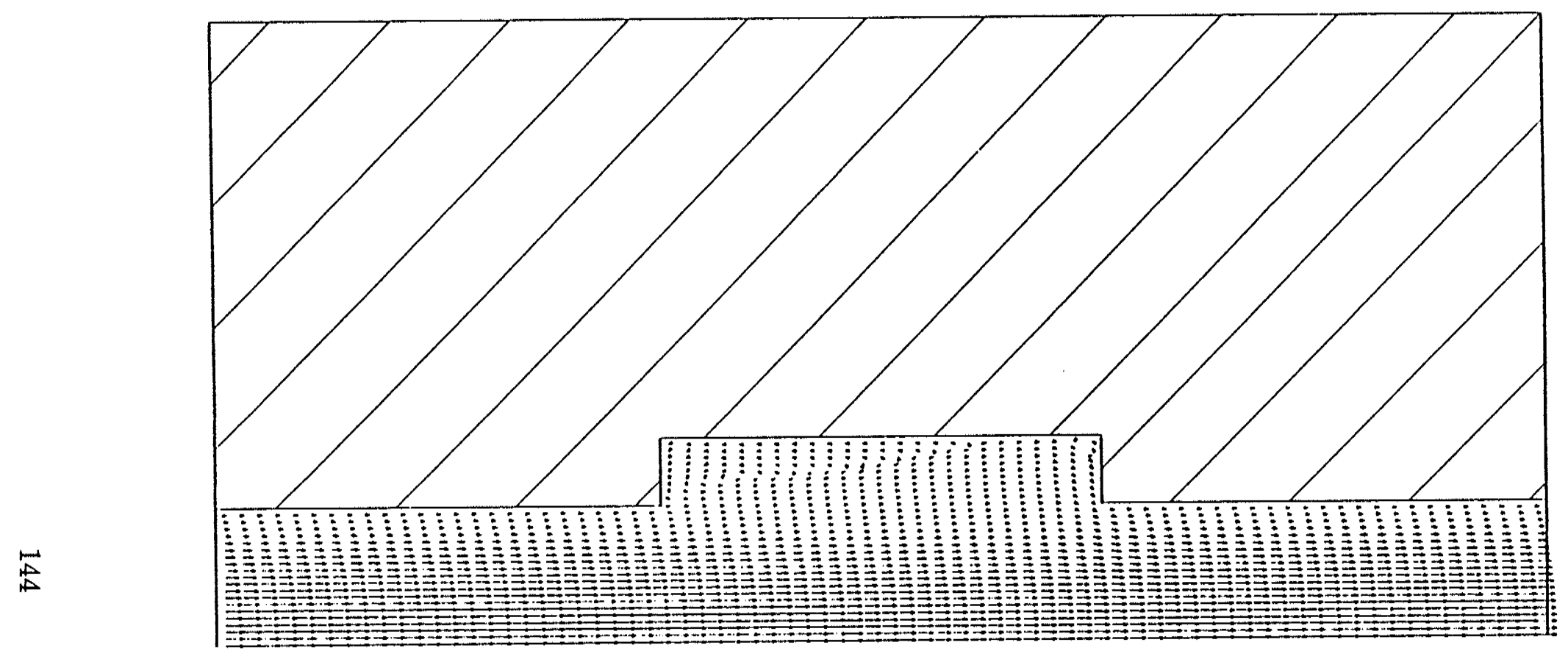

(C). $R e_{d}=80$

Fig. 8.10. Velocity field for the RUC of $D / d=1.5, L_{t} / L_{p}=2$ and $L / d=4.875$. 


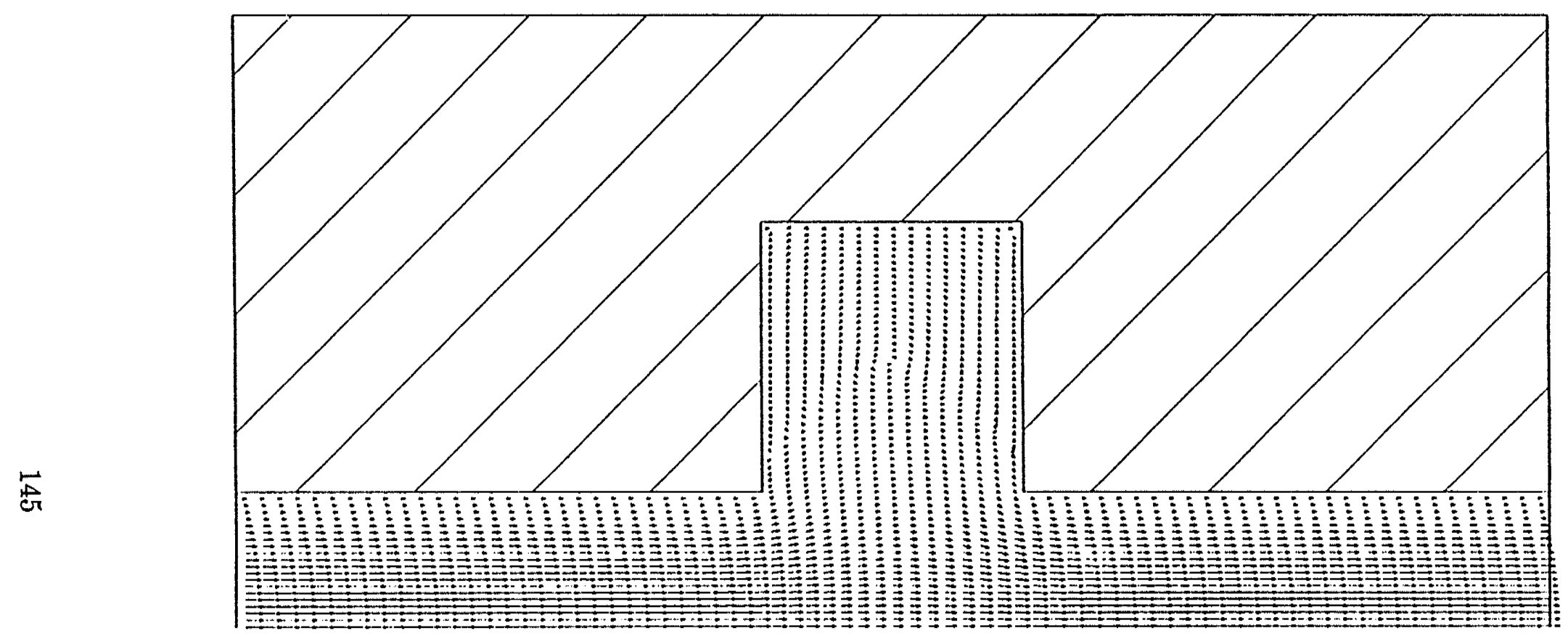

(A) $\cdot R e_{d}=1$

Fig. 8.11. Velocity field for the RUC of $D / d=3, L_{t} / L_{p}=4$ and $L / d=4.875$. 


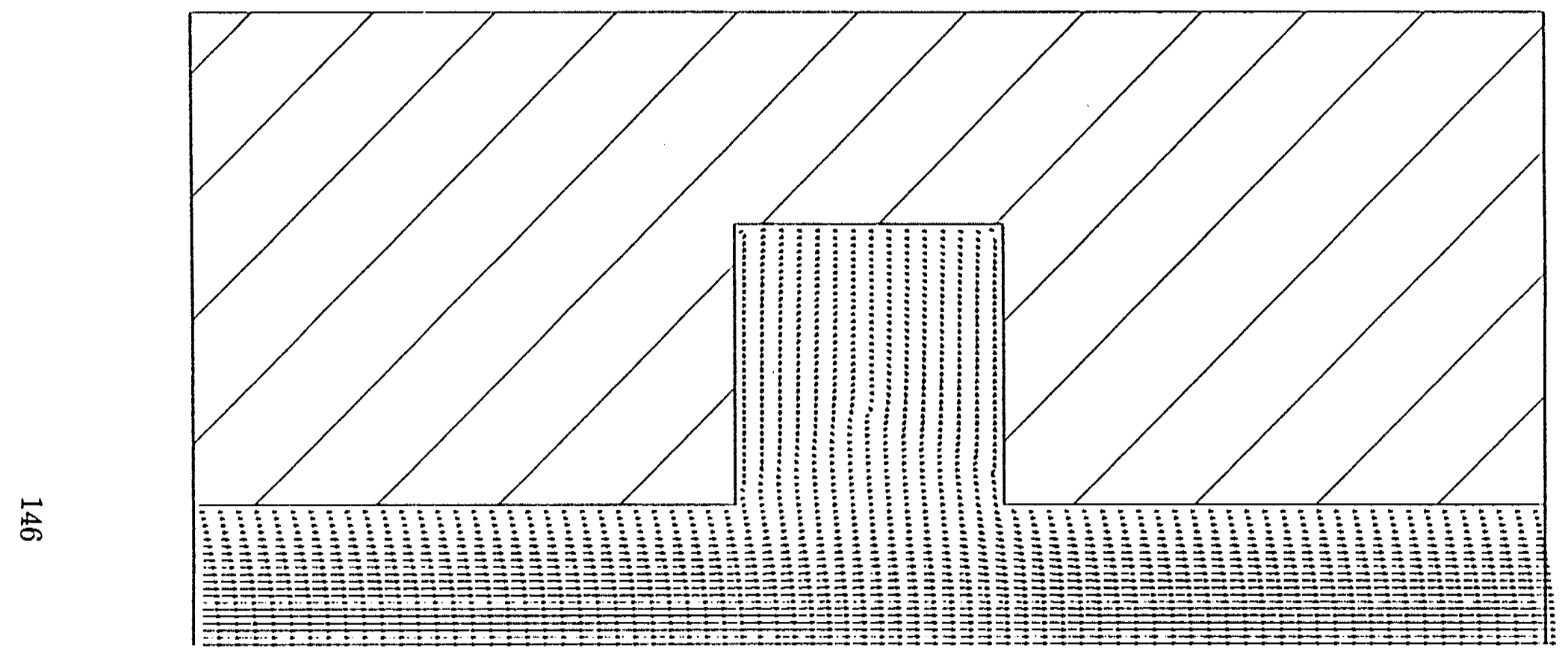

(B) $\cdot R e_{d}=20$

Fig. 8.11. Velocity field for the RUC of $D / d=3, L_{t} / L_{p}=4$ and $L / d=4.875$. 


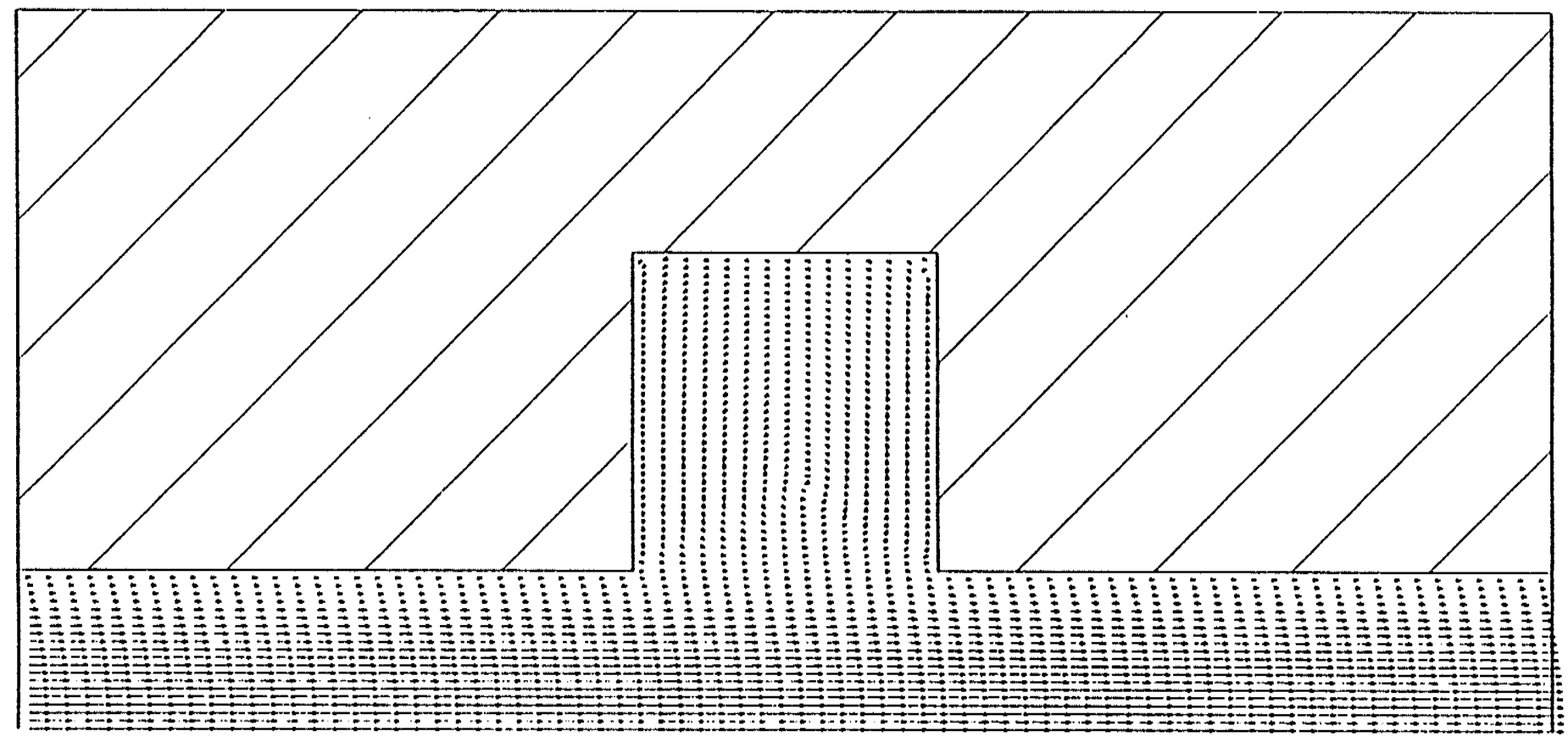

(C) $R e_{d}=80$

Fig. 8.11. Velocity field for the RUC of $D / d=3, L_{t} / L_{p}=4$ and $L / d=4.875$. 


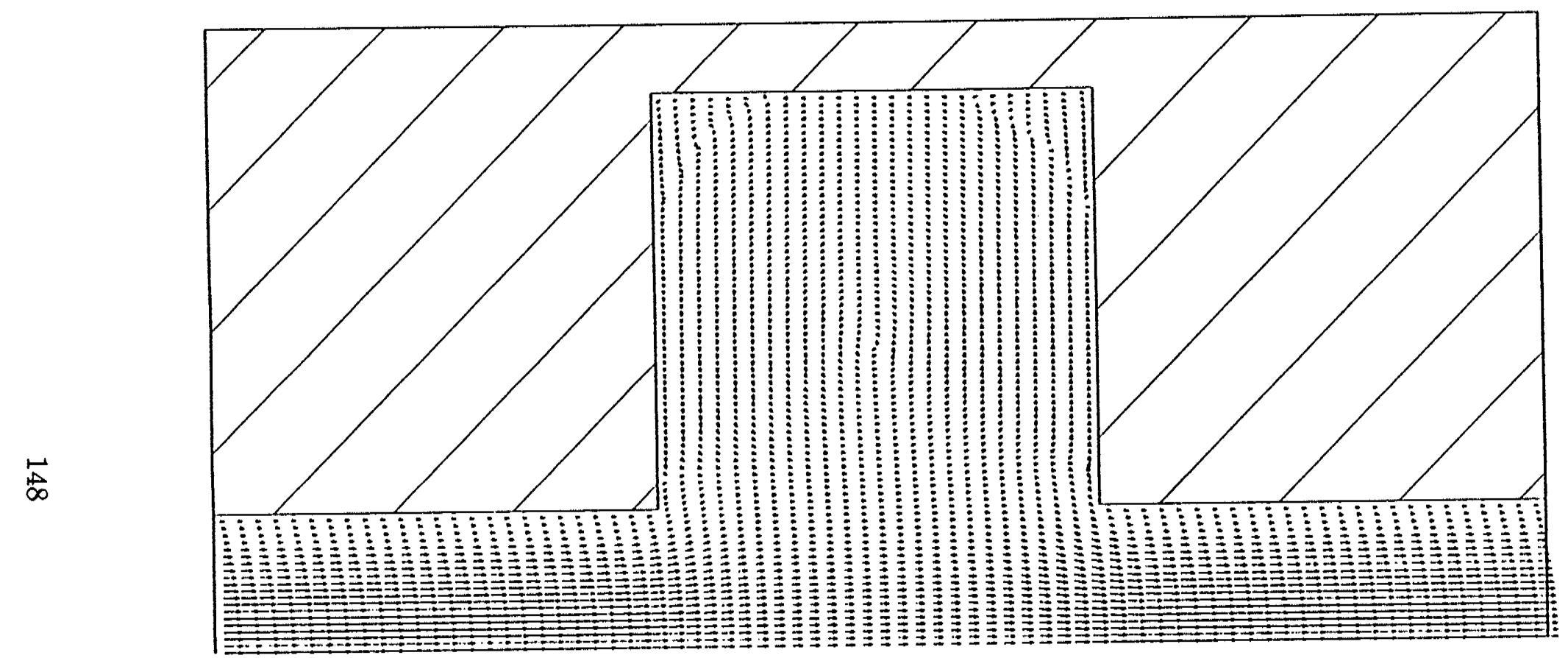

(A) $\cdot R e_{d}=1$

Fig. 8.12. Velocity field for the RUC of $D / d=4, L_{t} / L_{p}=2$ and $L / d=4.875$. 


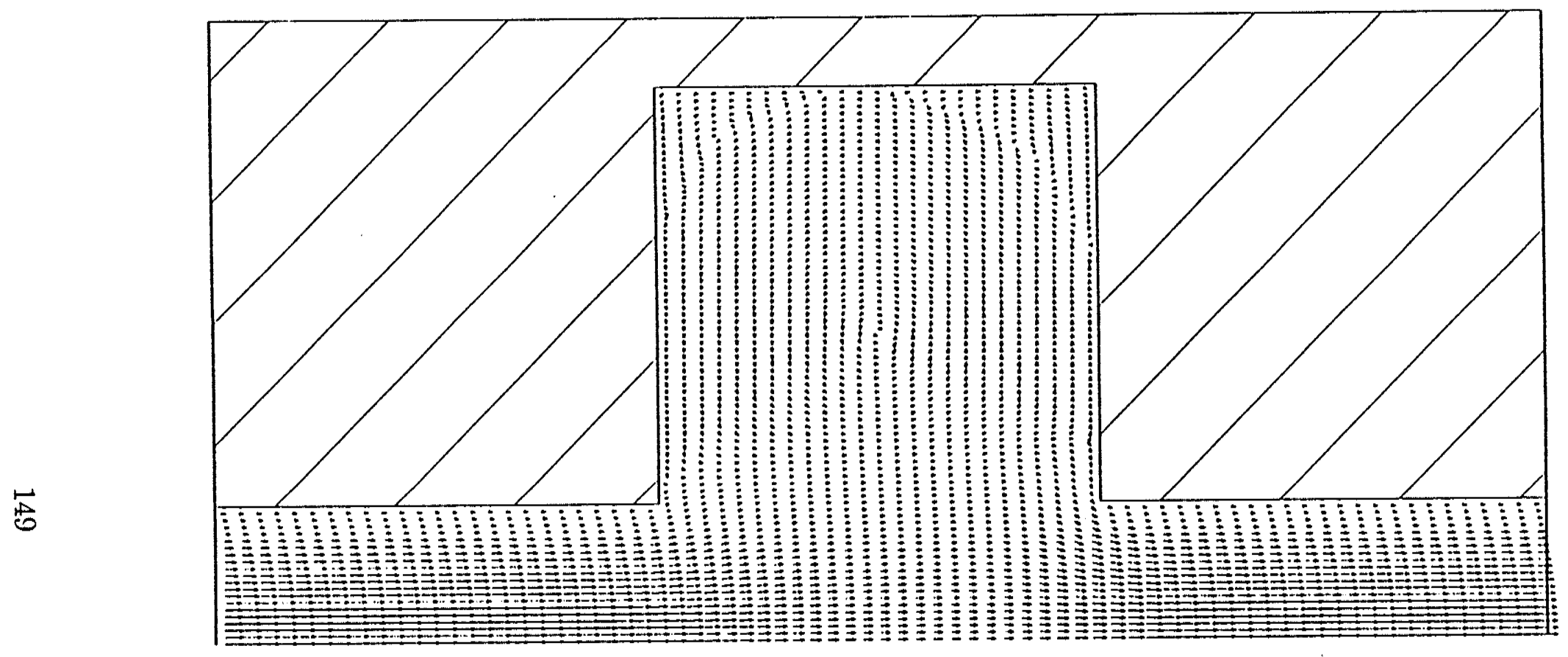

(B). $R e_{d}=10$

Fig. 8.12. Velocity field for the RUC of $D / d=4, L_{t} / L_{p}=2$ and $L / d=4.875$. 


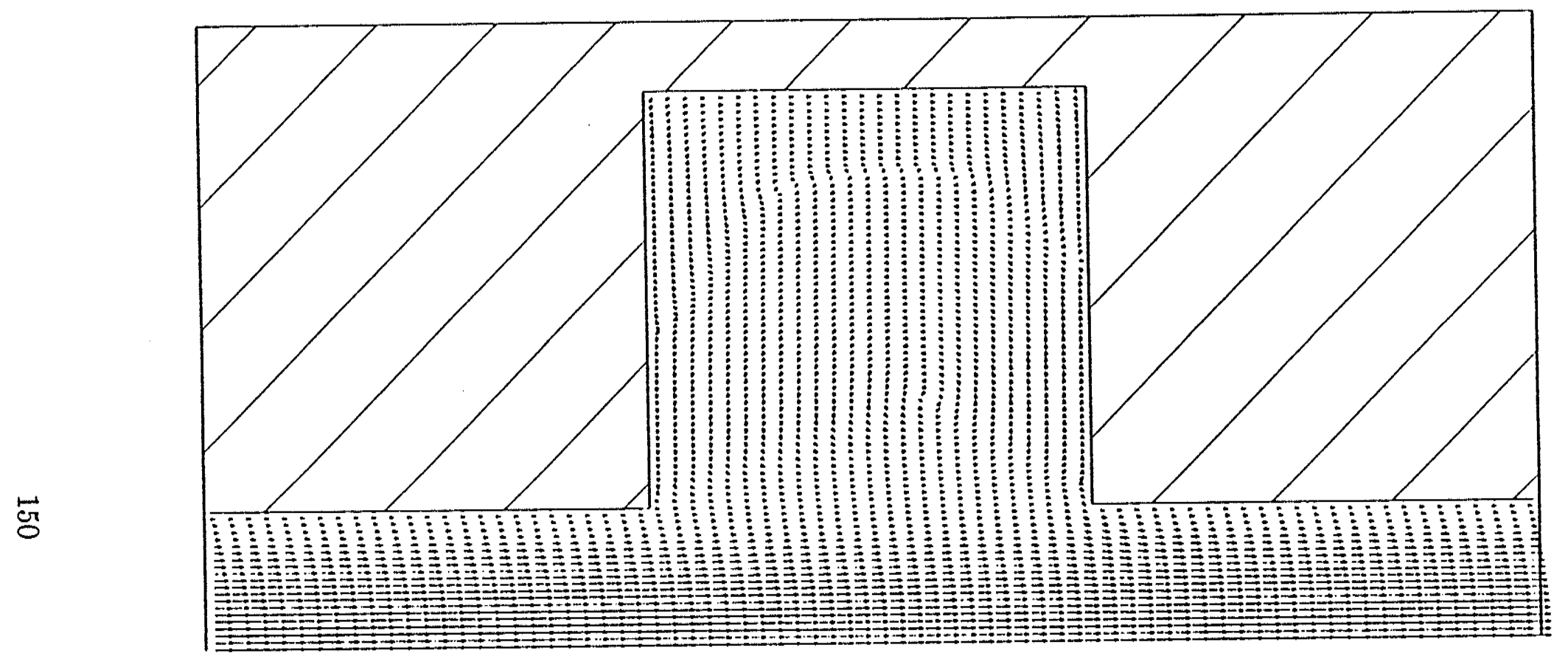

(C). $R e_{d}=70$

Fig. 8.12. Velocity field for the RUC of $D / d=4, L_{t} / L_{p}=2$ and $L / d=4.875$. 


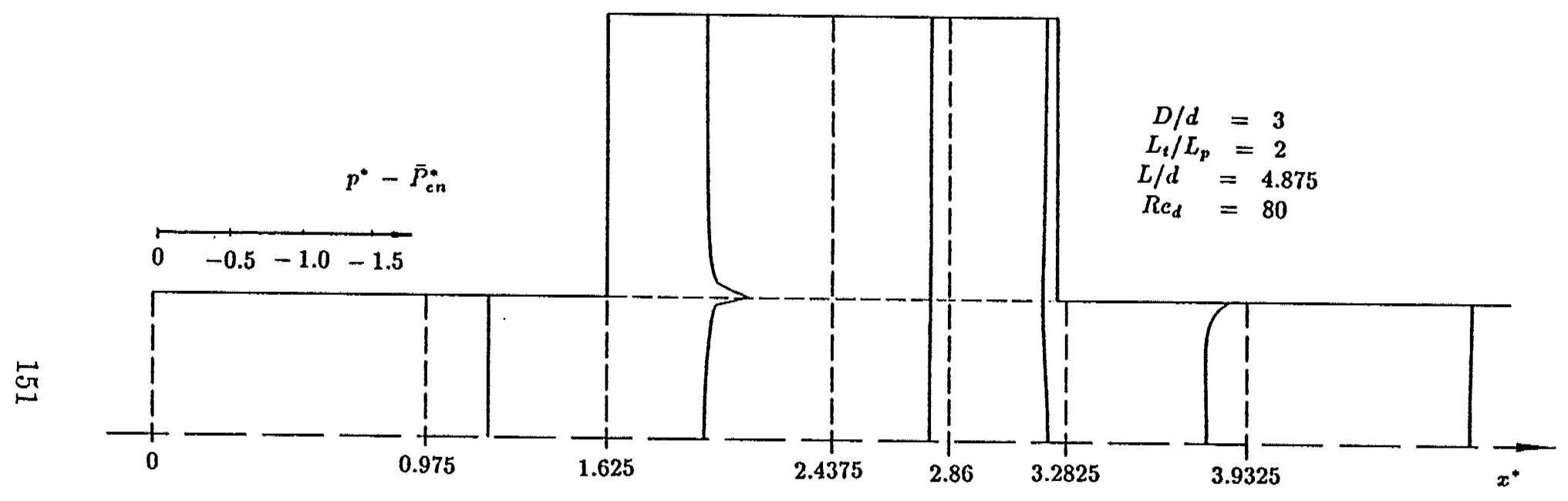

Fig. 8.13. Pressure distributions at representative axial positions for the RUC of $D / d=3, L_{t} / L_{p}=2$ and $L / d=4.875$. 


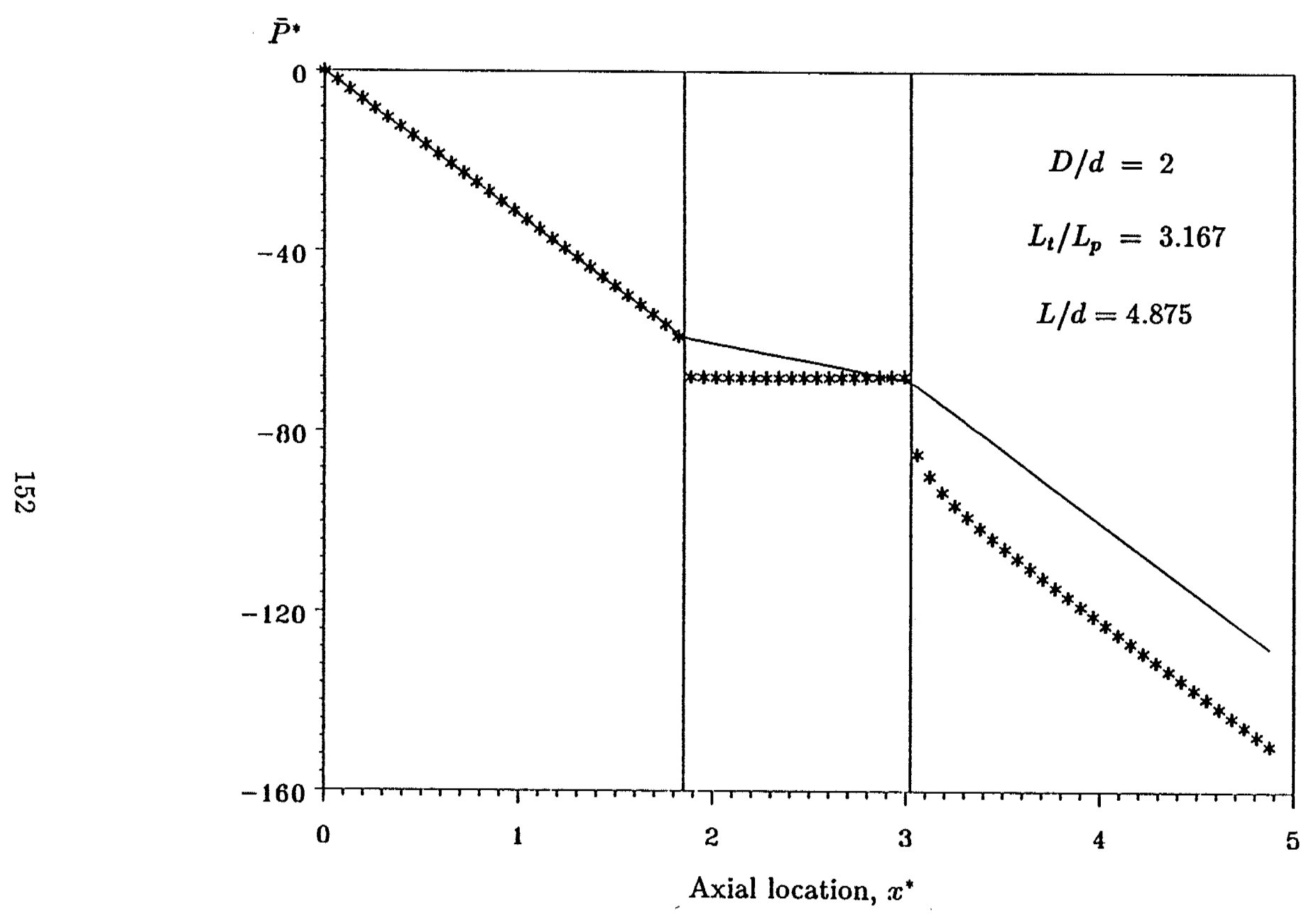

(A). $R e_{d}=1$

Fig. 8.14. Mean pressure distributions. 


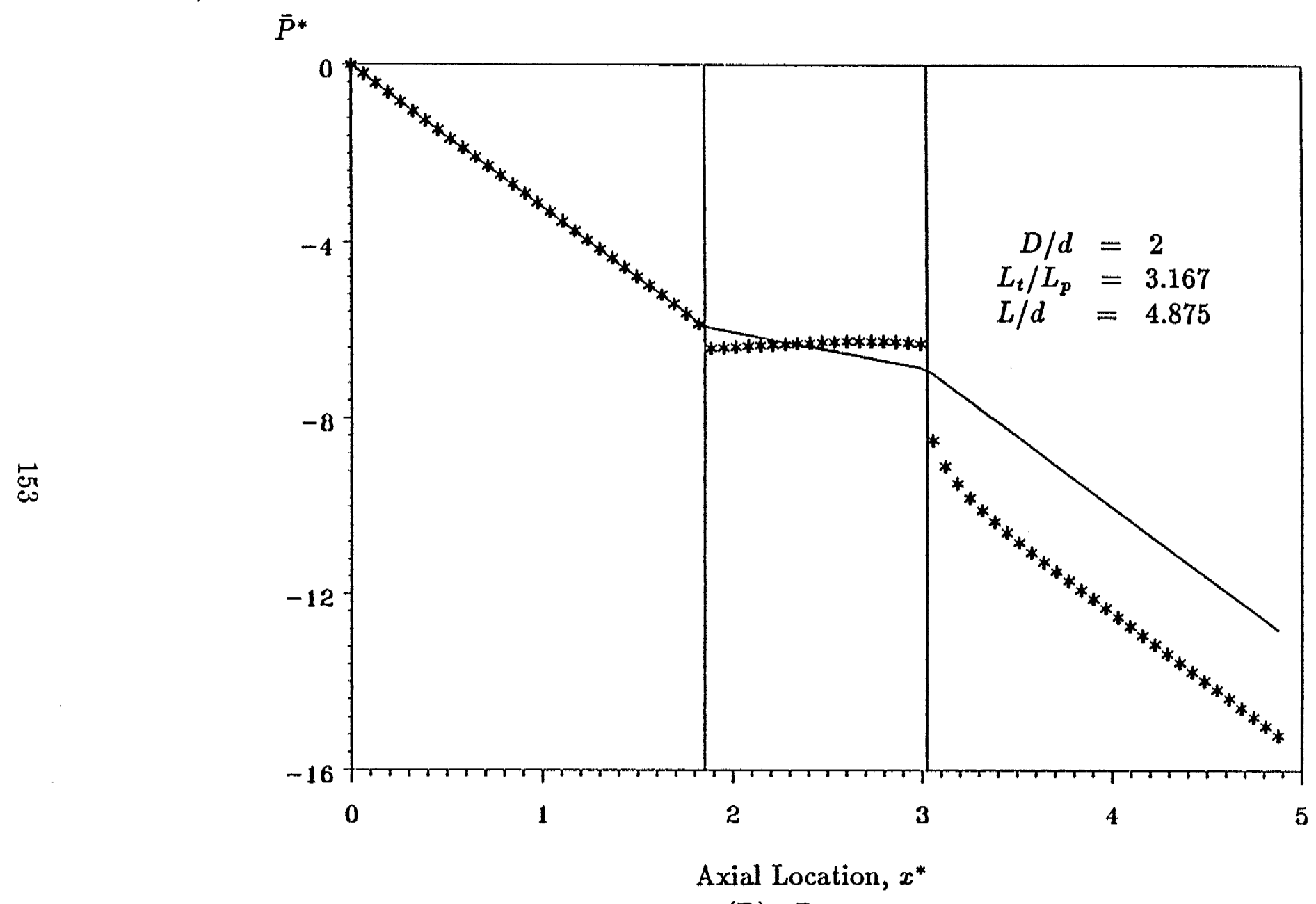

(B). $R e_{d}=10$

Fig. 8.14. Mean pressure distributions. 


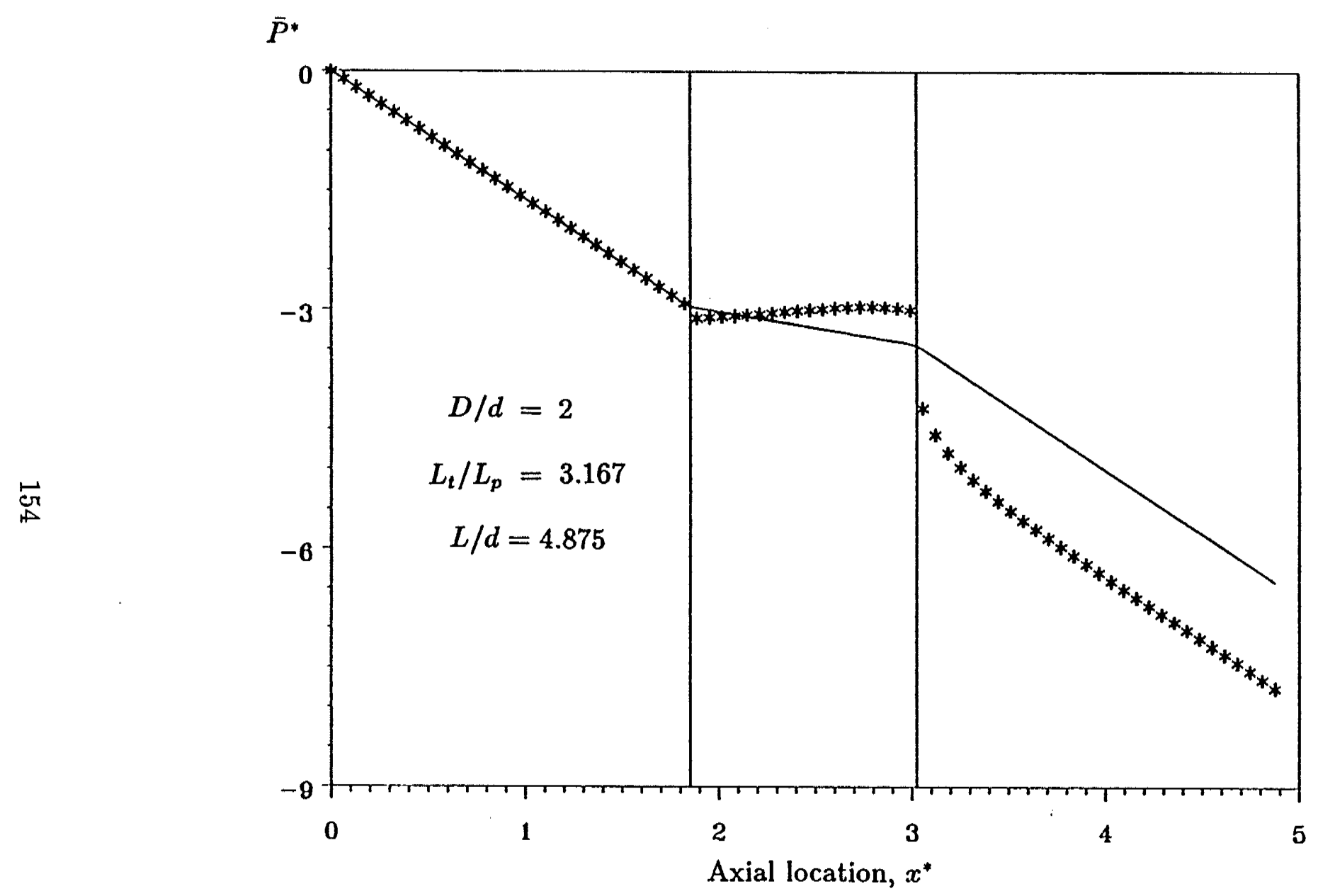

(C). $R e_{d}=20$

Fig. 8.14. Mean pressure distributions. 


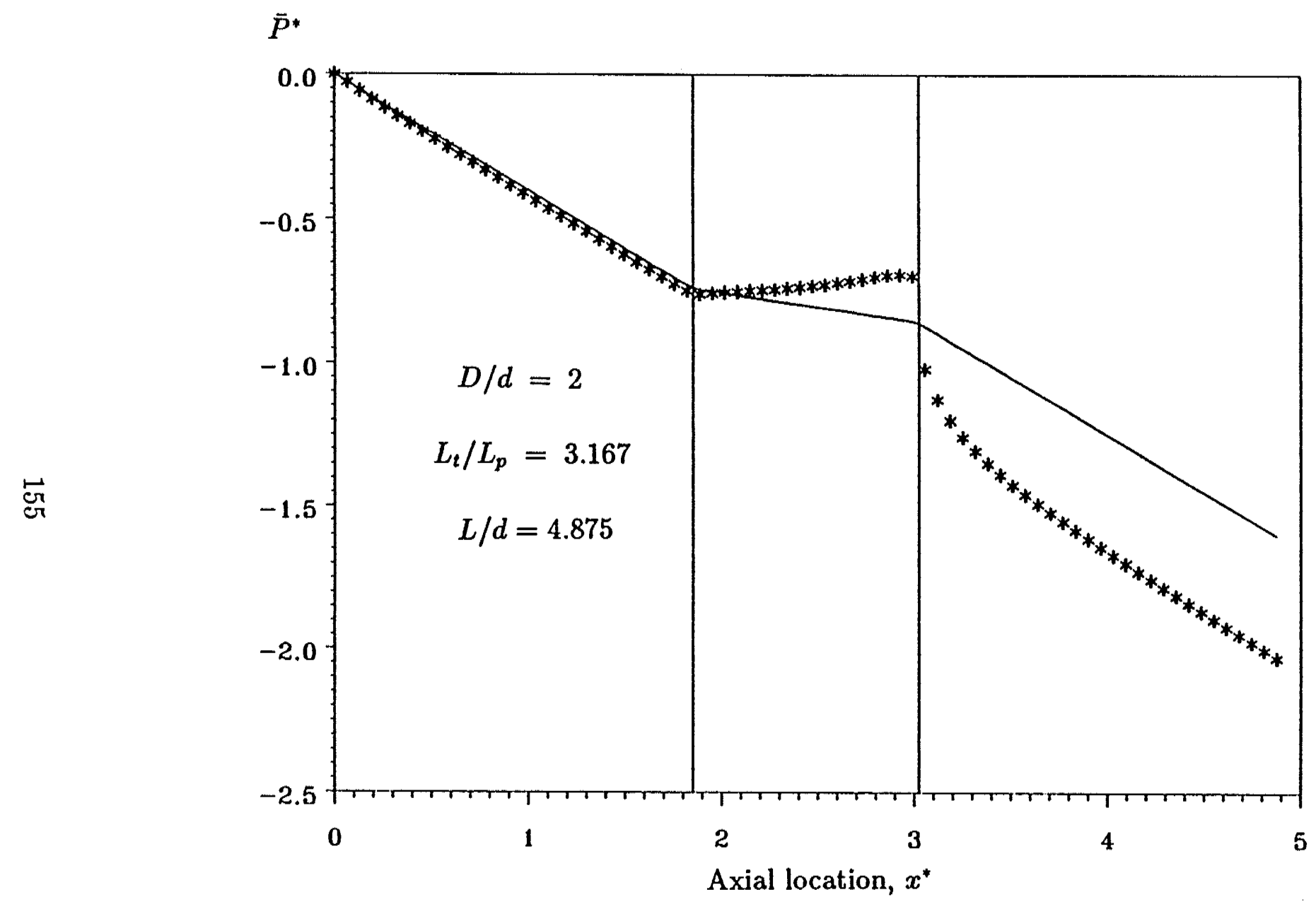

(D). $R e_{d}=80$

Fig. 8.14. Mean pressure distributions. 


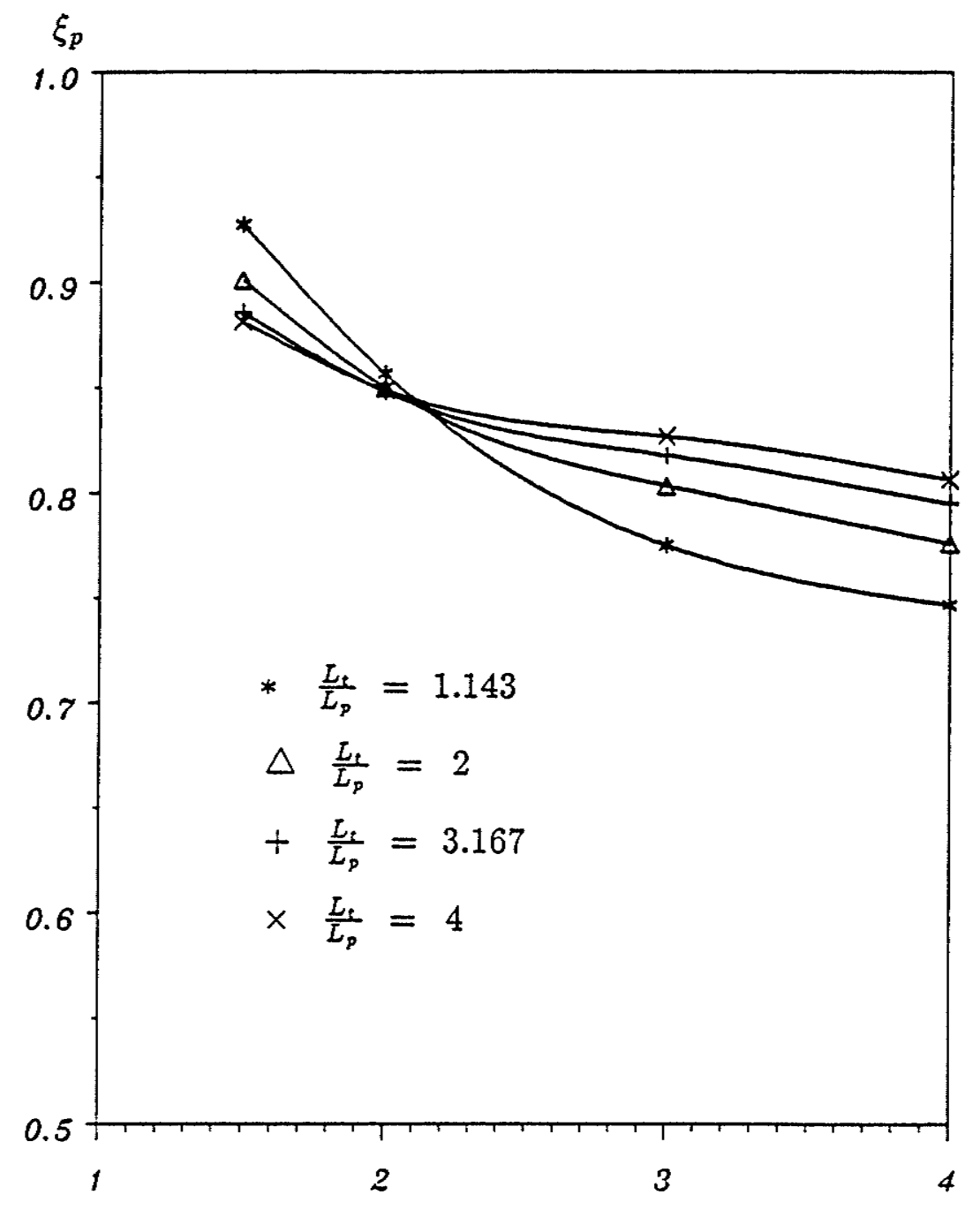

Diameter Ratio, $D / d$

Fig. 8.15. Effects of length ratio and diameter ratio on $\xi_{p}$ for $R e_{d}=10$, $L / d=4.875$. 


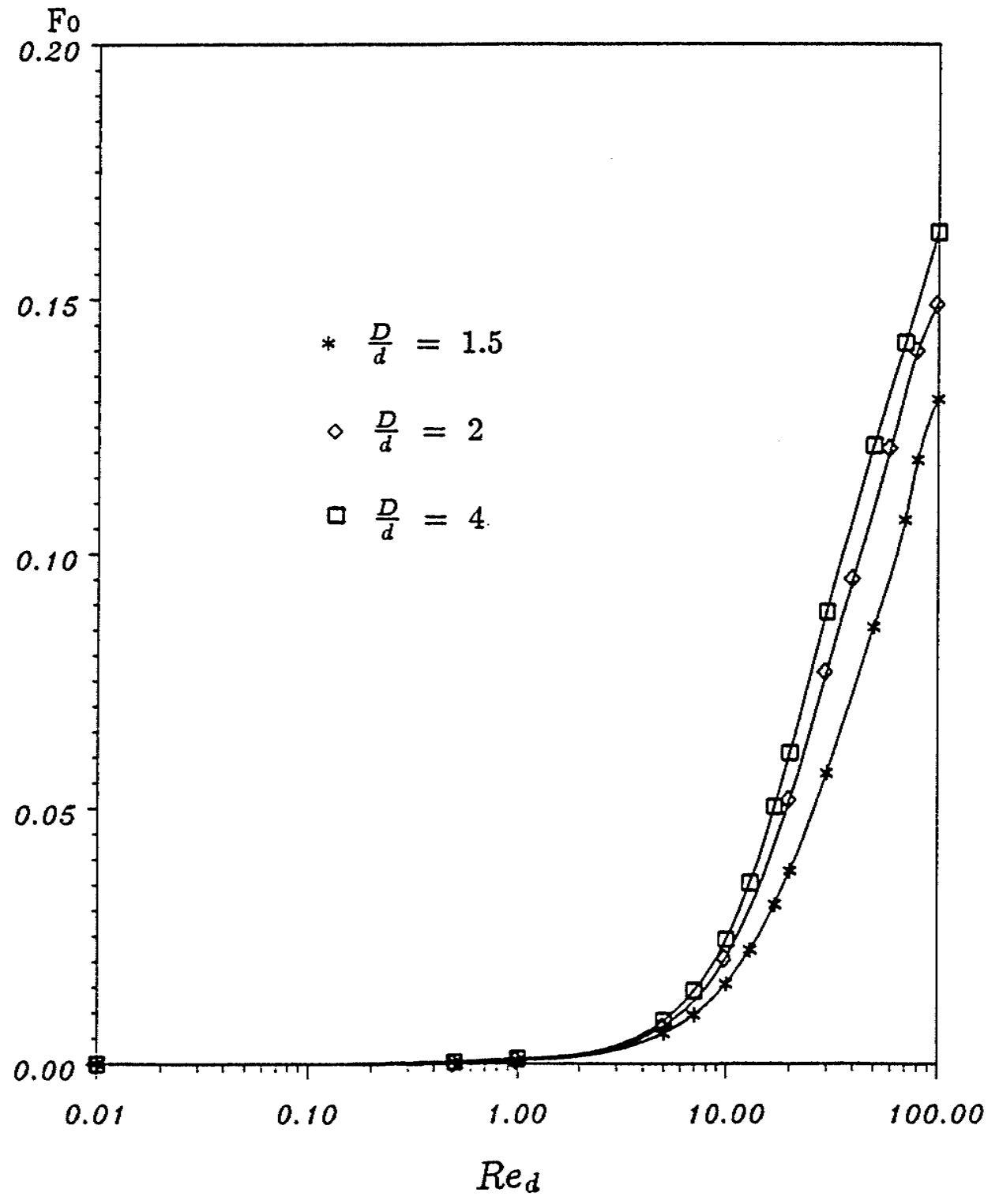

Fig. 8.16. Variations of Forchheimer number with Reynolds number for the RUC of $L_{t} / L_{p}=2$ and $L / d=4.875$. 


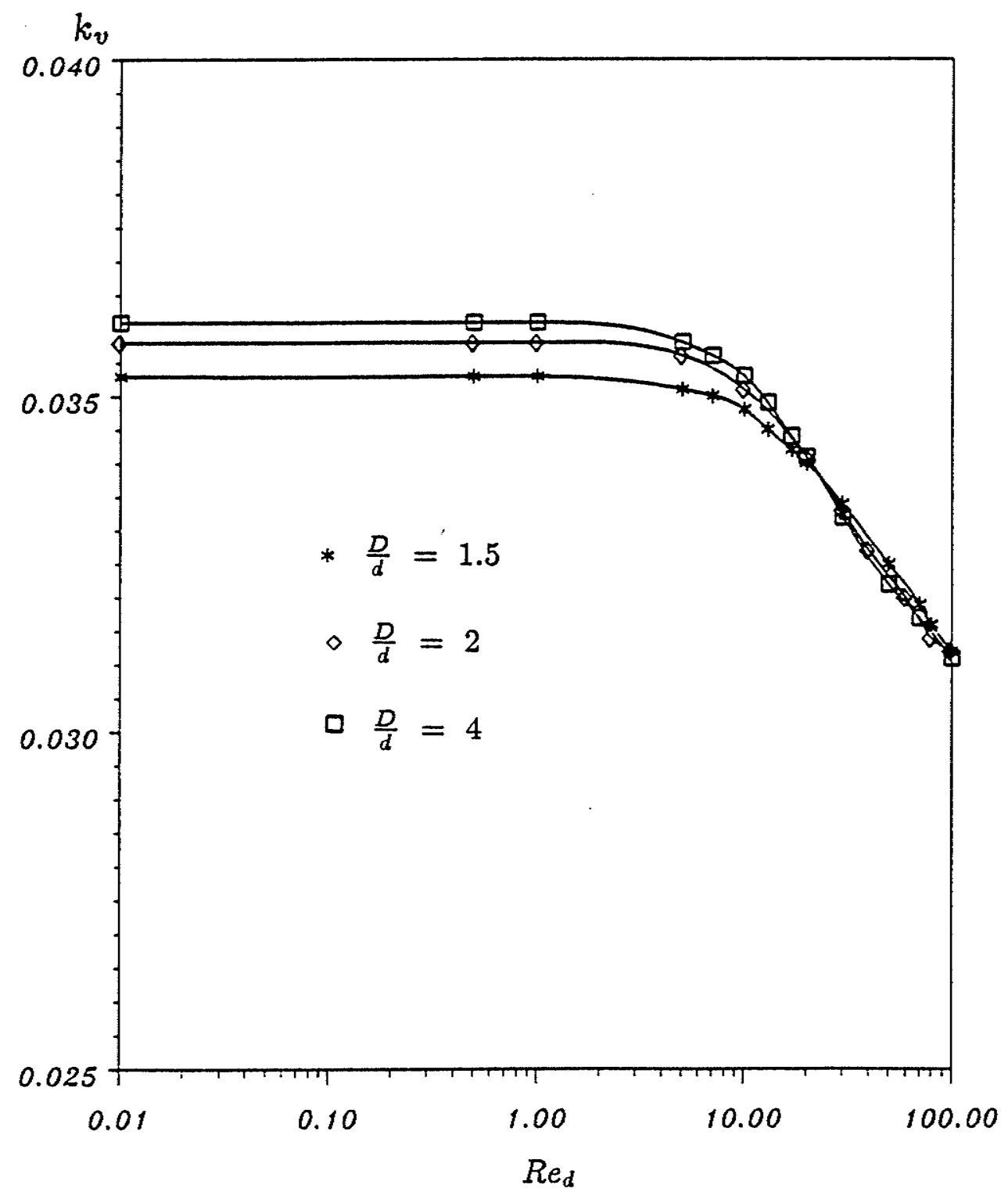

Fig. 8.17. Variations of the velocity-dependent permeability with $R e_{d}$ for the RUC of $L_{t} / L_{p}=2$ and $L / d=4.875$. 


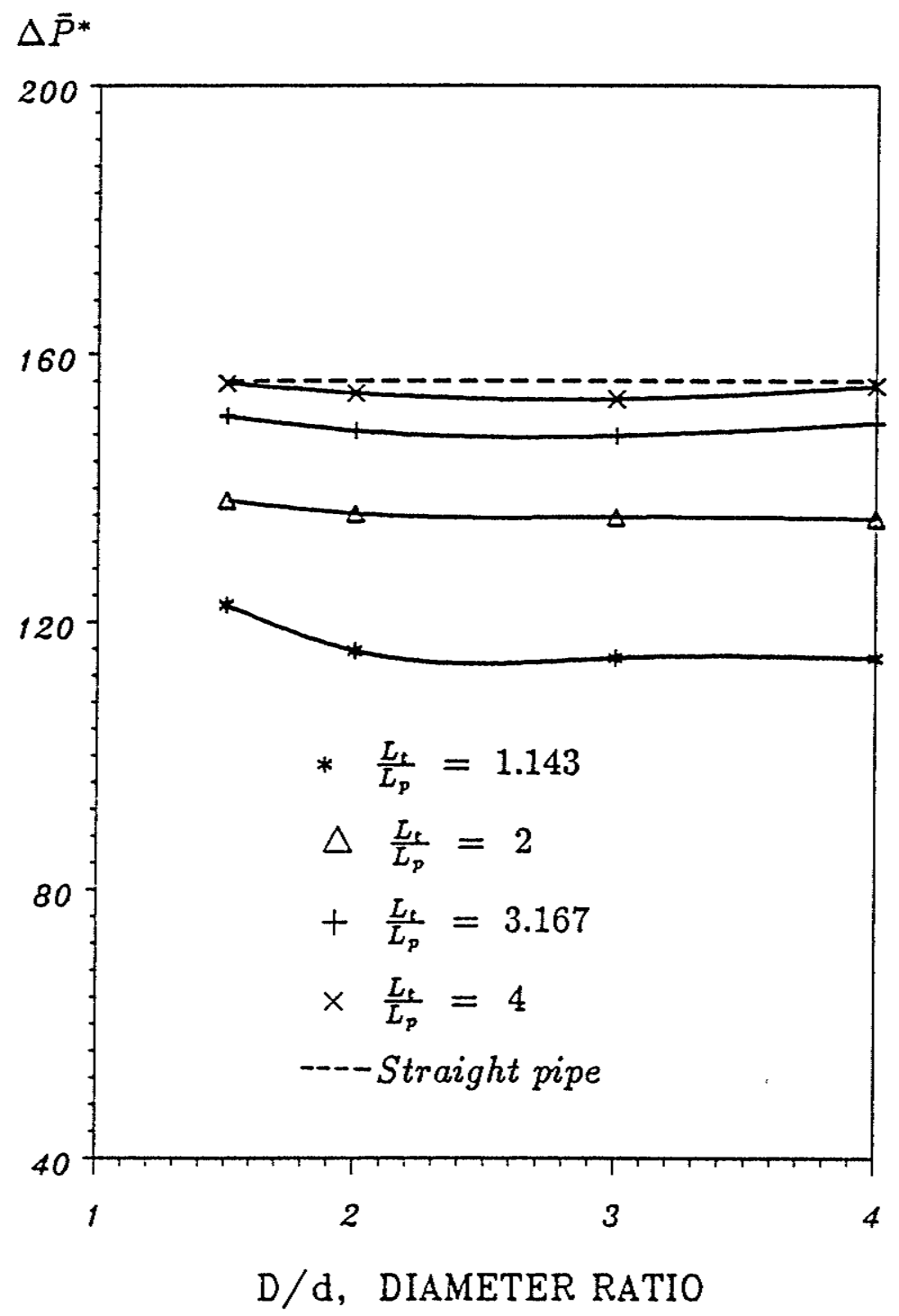

(A). $R e_{d}=1$

Fig. 8.18. Variations of the average pressure loss for the RUC of $L / d=4.875$. 


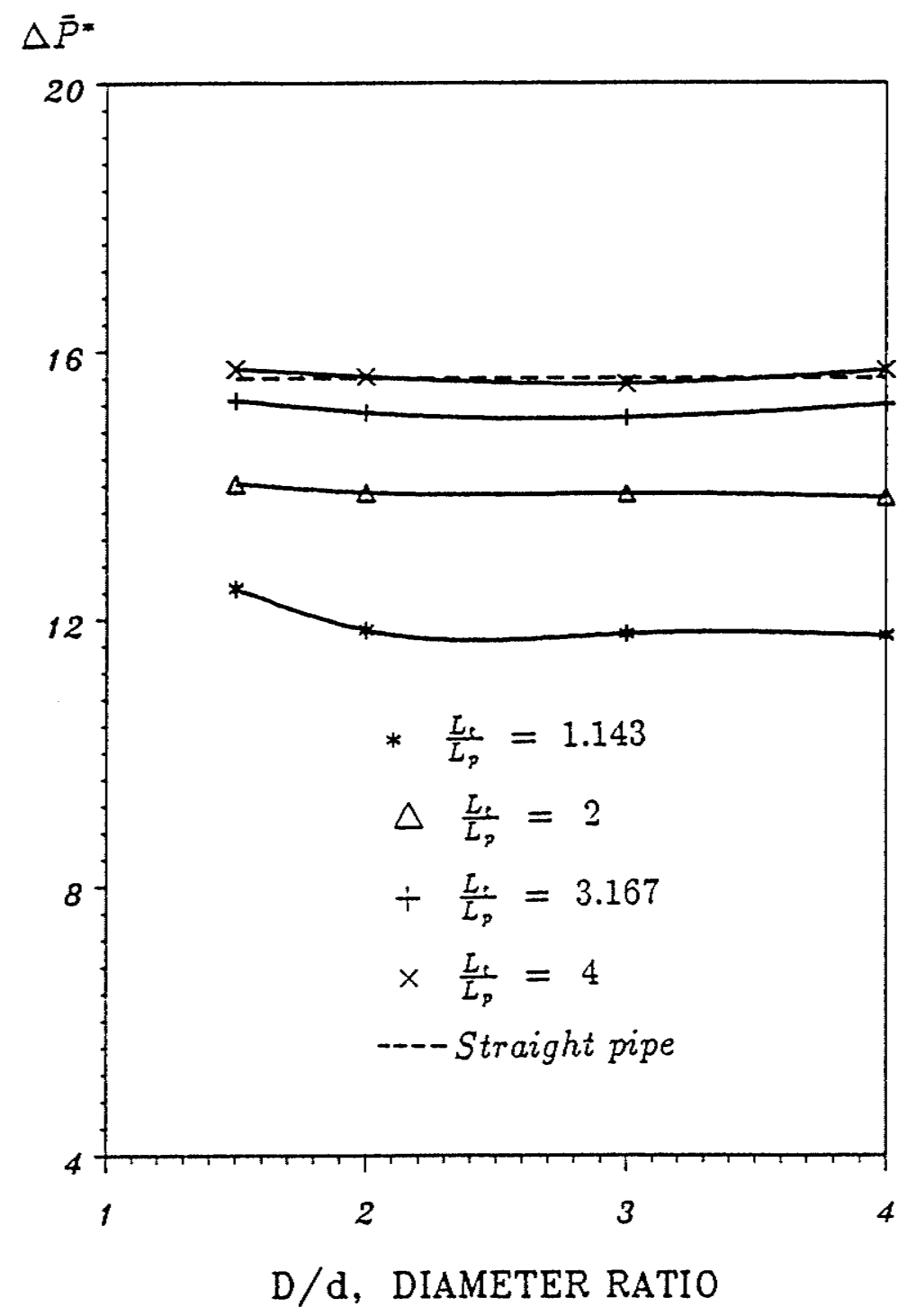

(B). $R e_{d}=10$

Fig. 8.18. Variations of the average pressure loss for the RUC of $L / d=4.875$. 


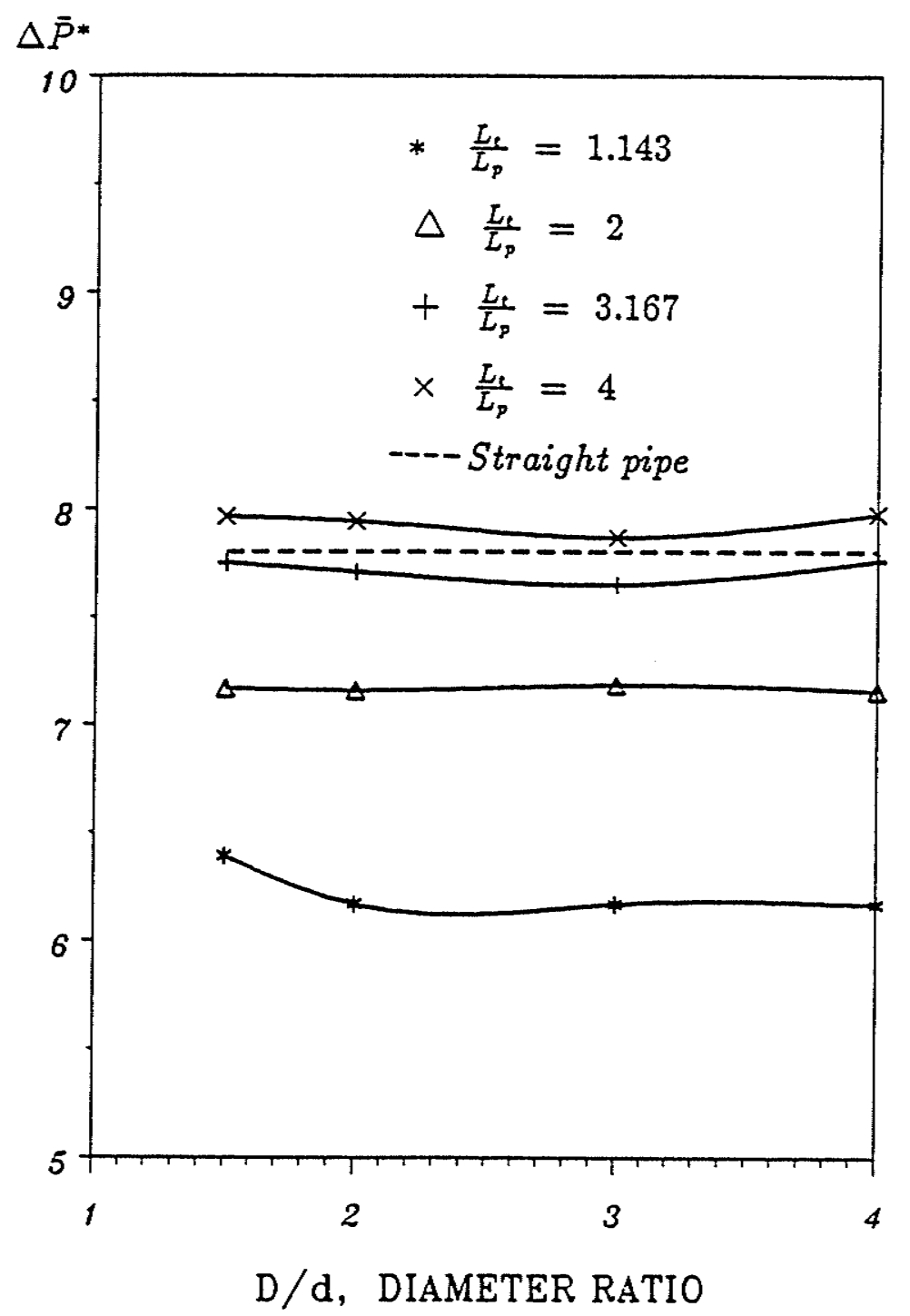

(C). $R e_{d}=20$

Fig. 8.18. Variations of the average pressure loss for the RUC of $L / d=4.875$. 


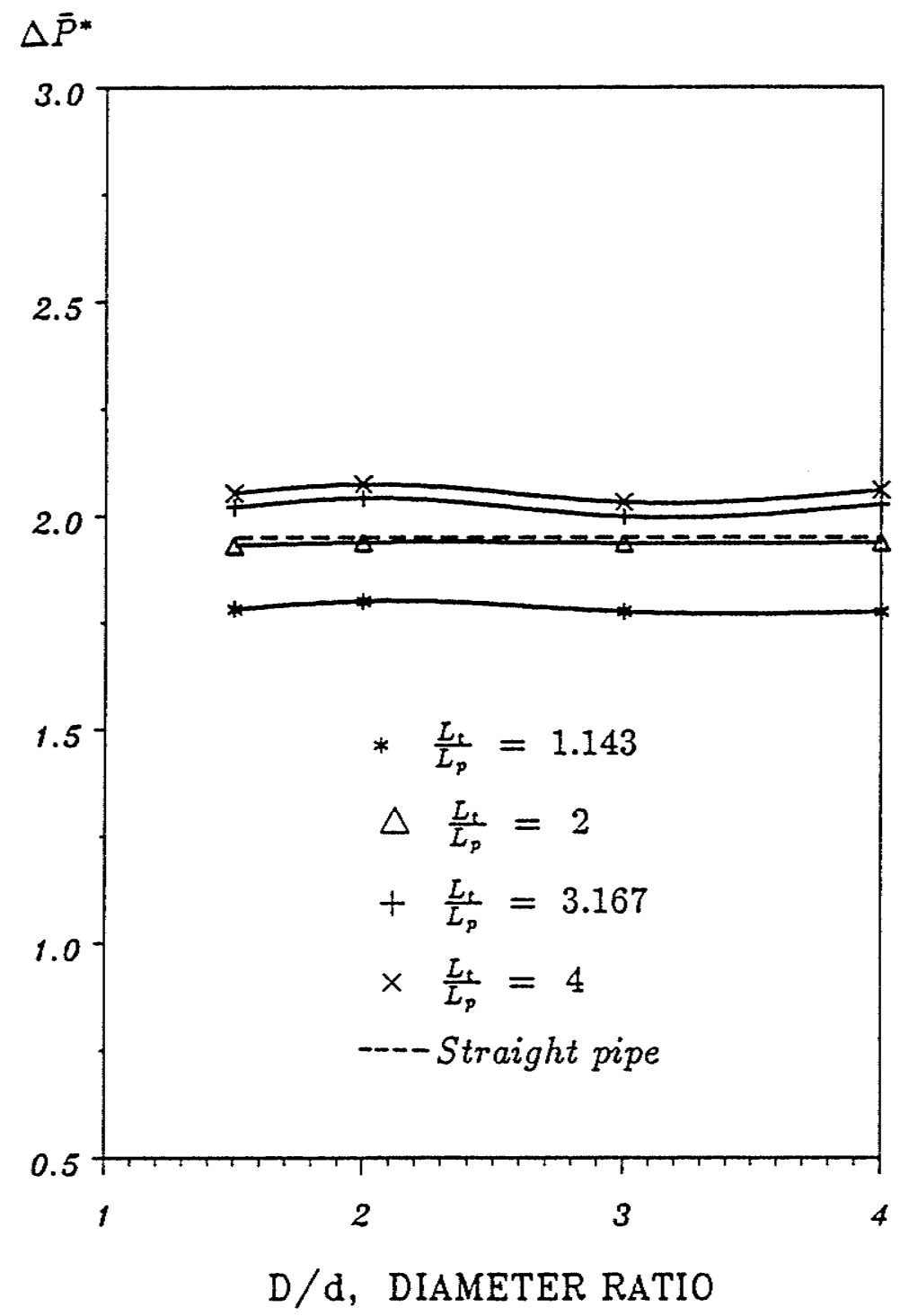

(D). $R e_{d}=80$

Fig. 8.18. Variations of the average pressure loss for the RUC of $L / d=4.875$. 


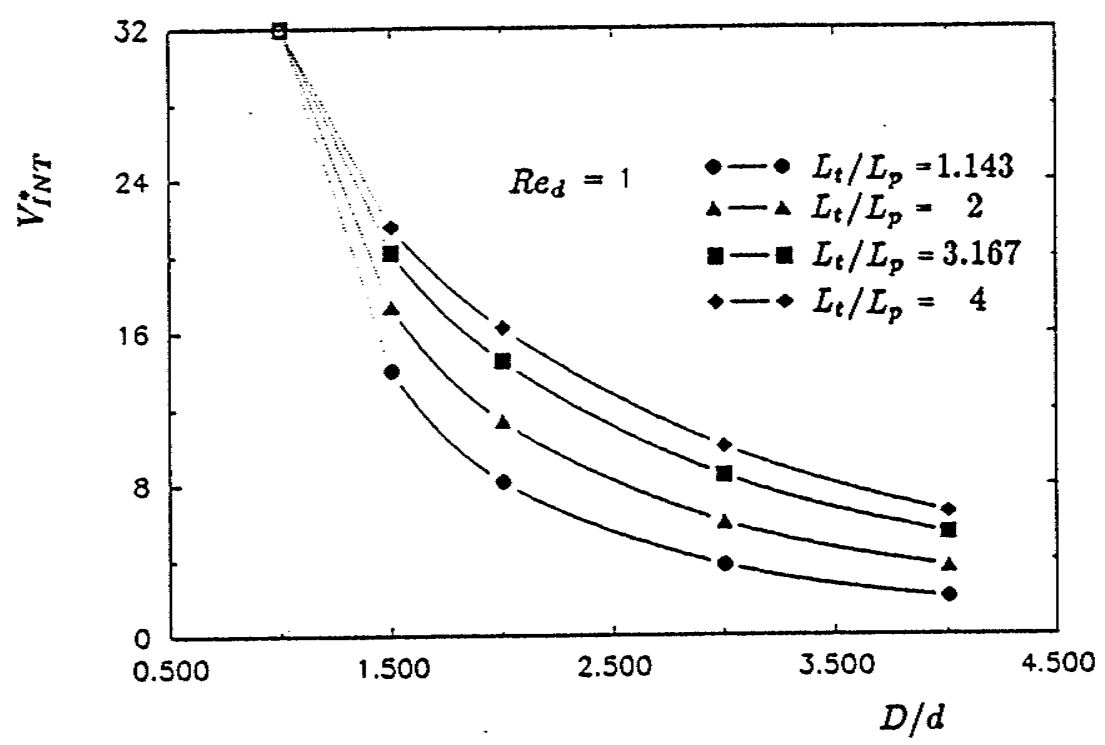

(A). $R e_{d}=1$

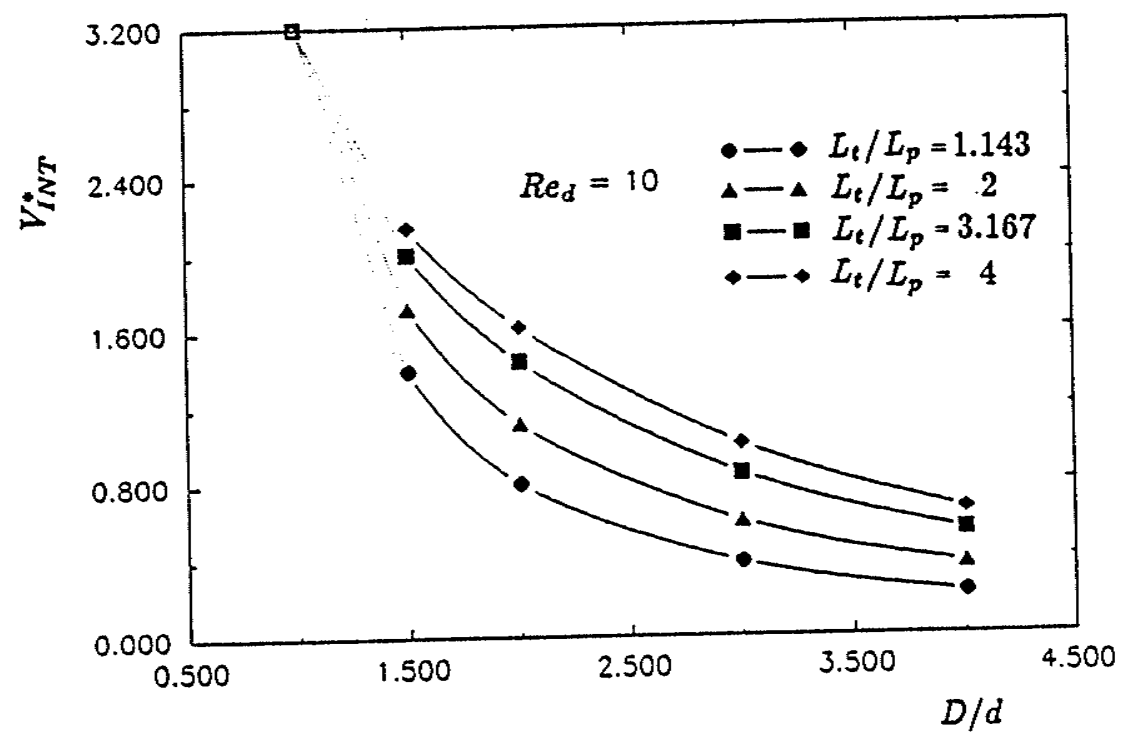

(B). $R e_{d}=10$

Fig. 8.19. Effects of the pore parameters on the viscous integral term for $L / d=4.875$. 


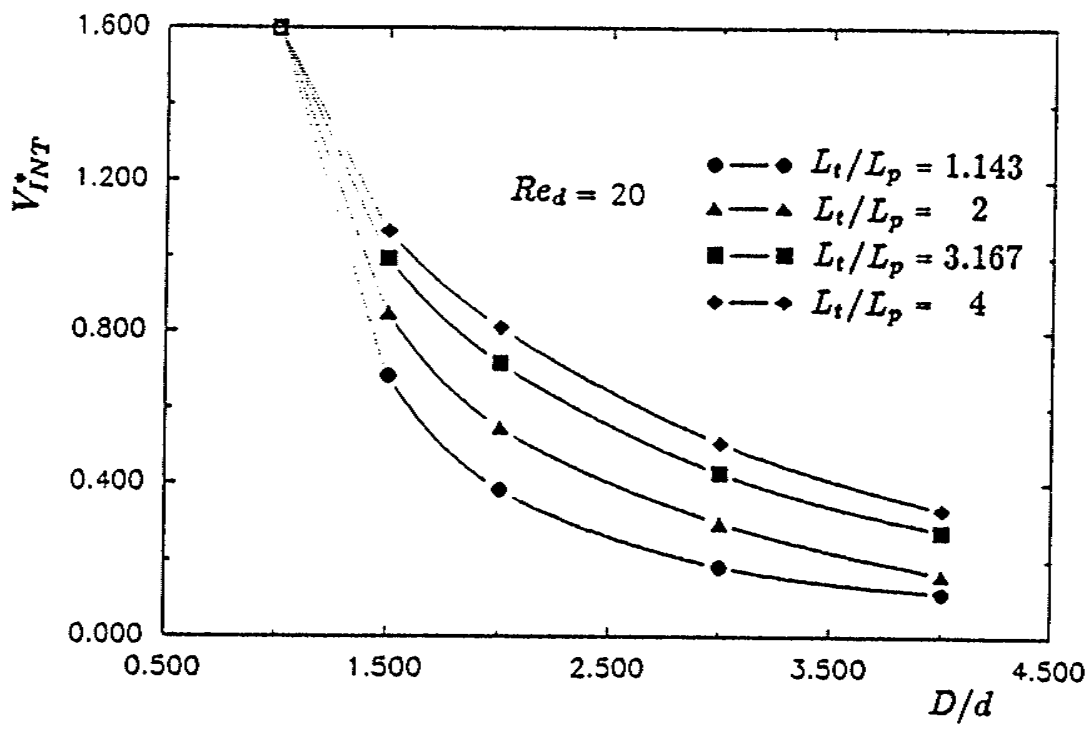

(C). $R e_{d}=20$

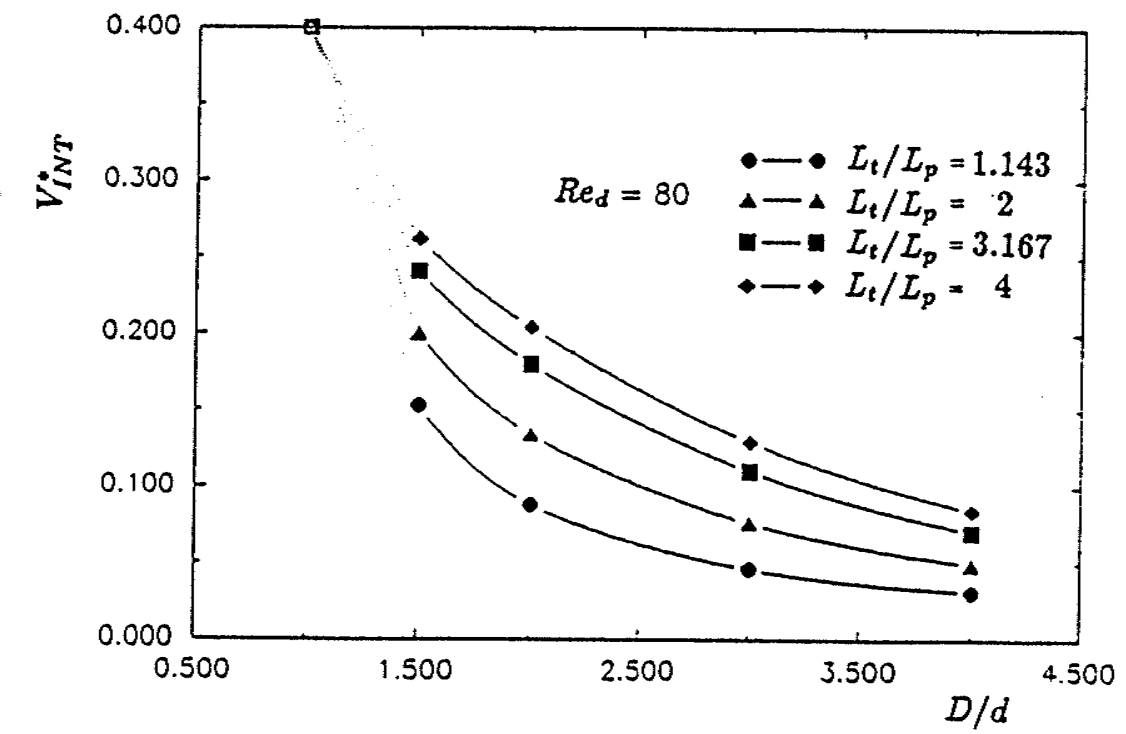

(D). $R e_{d}=80$

Fig. 8.19. Effects of the pore parameters on the viscous integral term for $L / d=4.875$. 


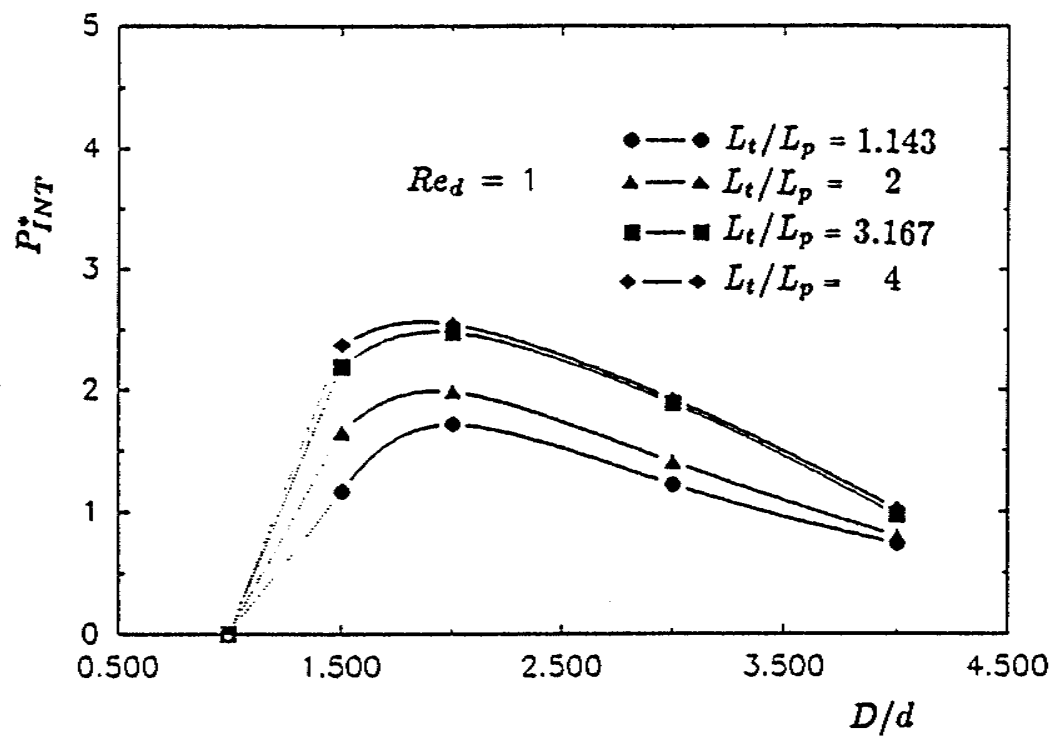

(A). $R e_{d}=1$

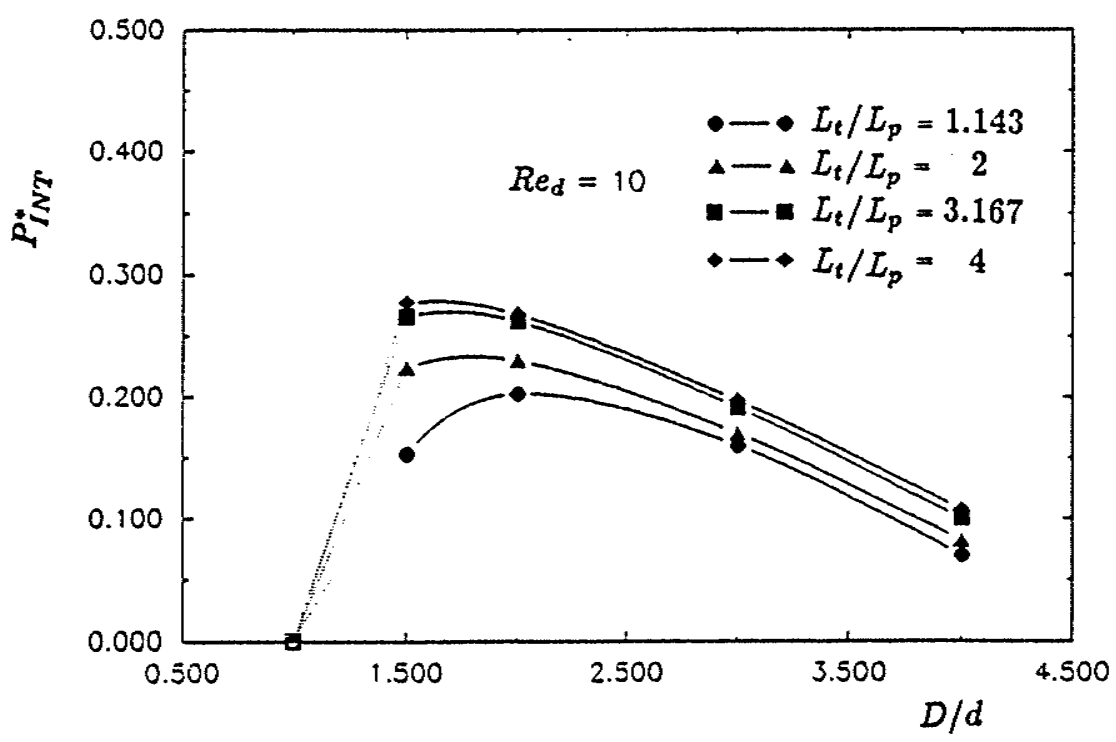

(B). $R e_{d}=10$

Fig. 8.20. Effects of the pore parameters on the pressure integral term for $L / d=4.875$. 


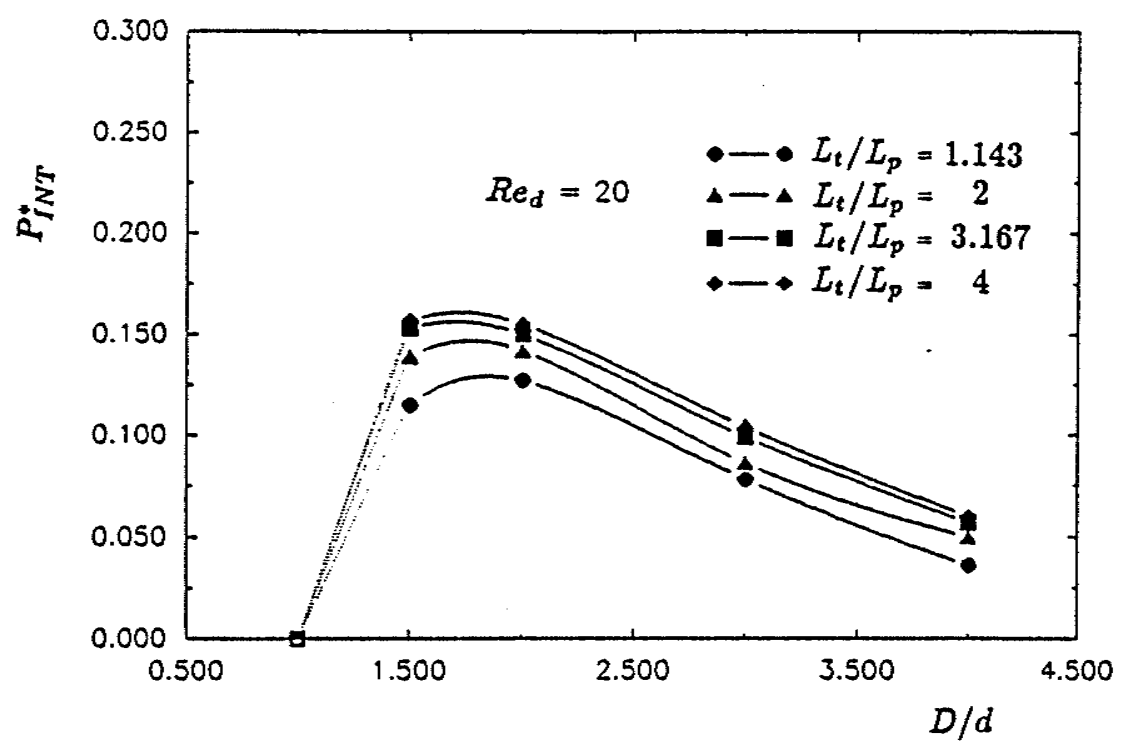

(C). $R e_{d}=20$

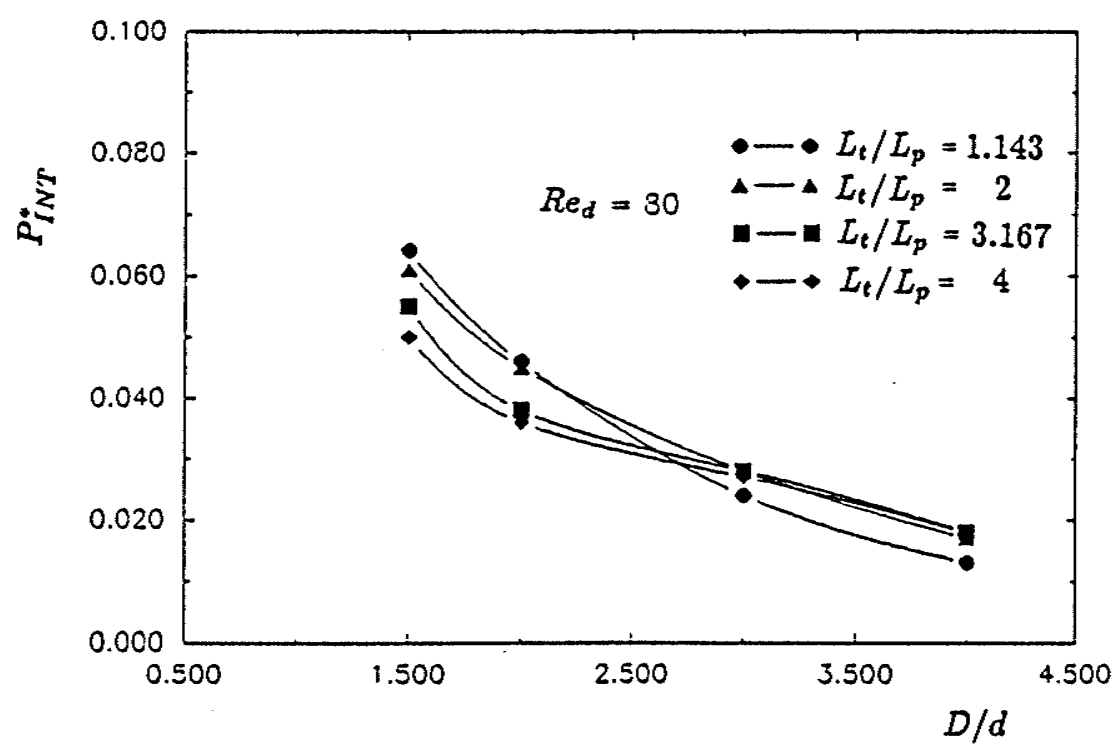

(D). $R e_{d}=80$

Fig. 8.20. Effects of the pore parameters on the pressure integral term for $L / d=4.875$. 


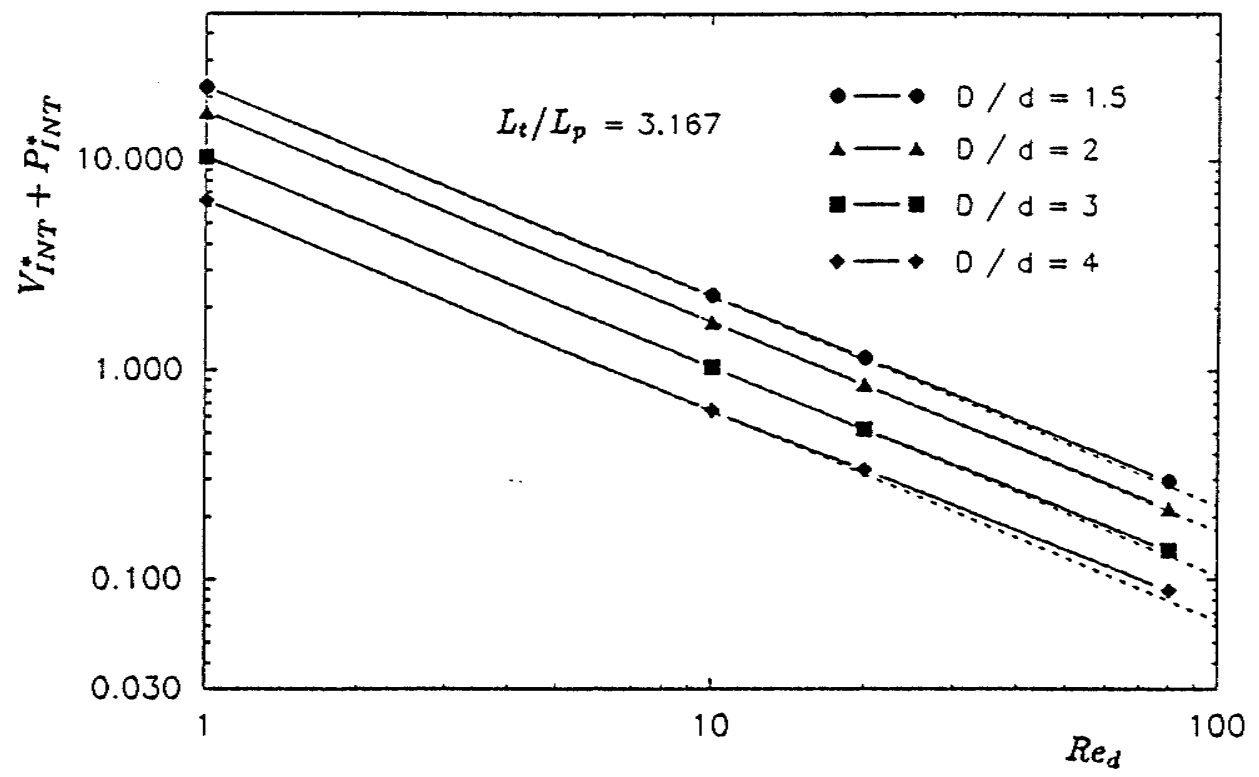

(A). $L_{t} / L_{p}=3.167$

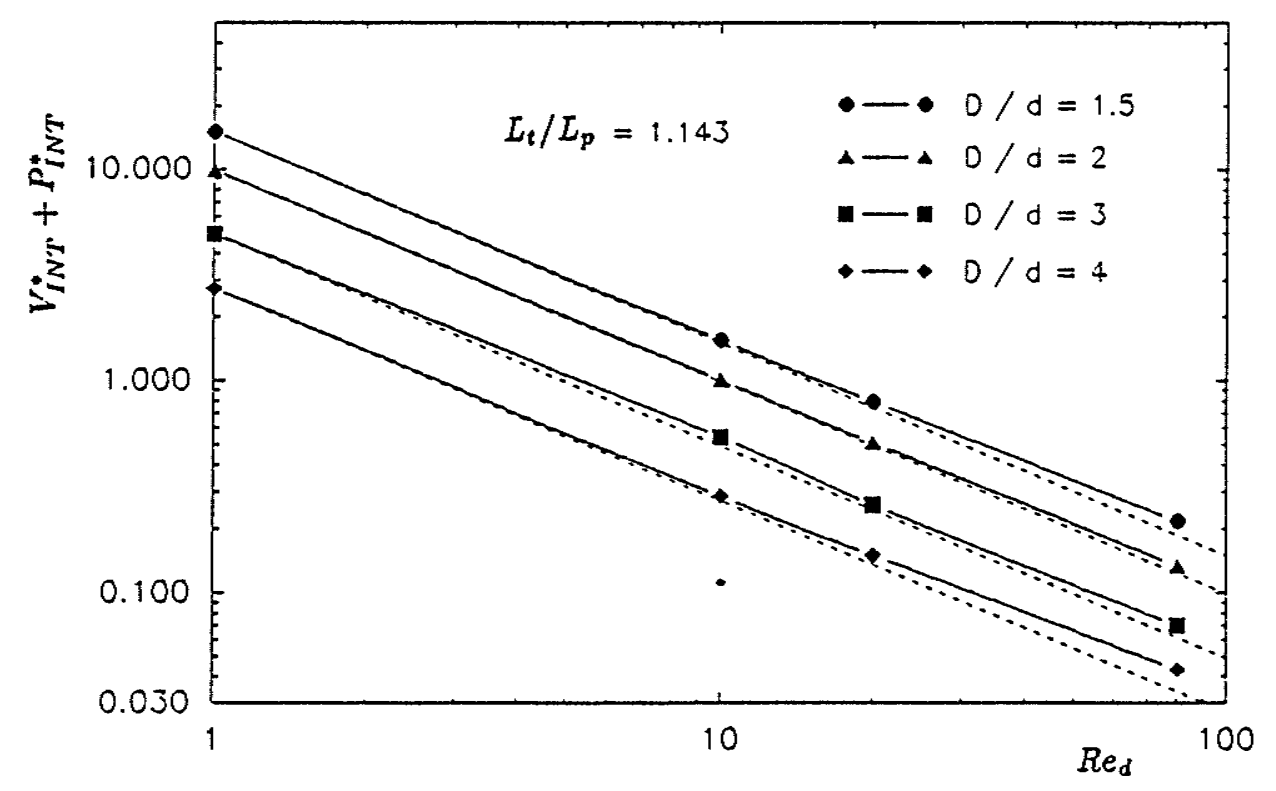

(B). $L_{t} / L_{p}=1.143$

Fig. 8.21. Effects of the pore Reynold number on the interfacial drag force for $L / d=4.875$. 


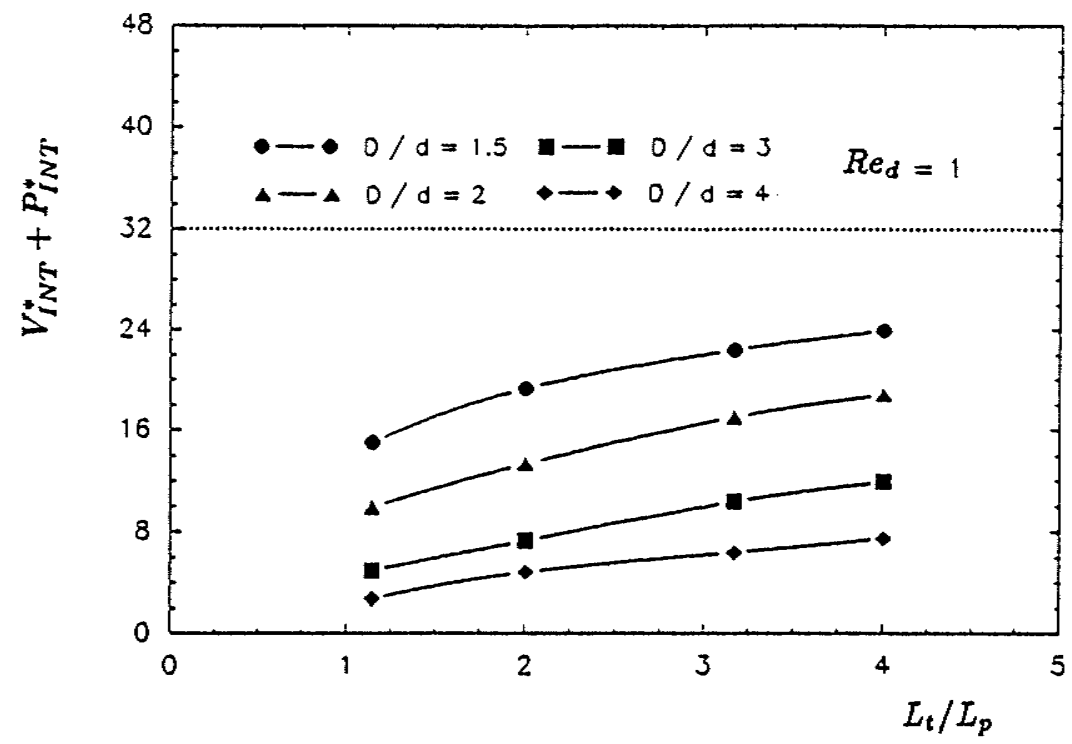

(A). $R e_{d}=1$

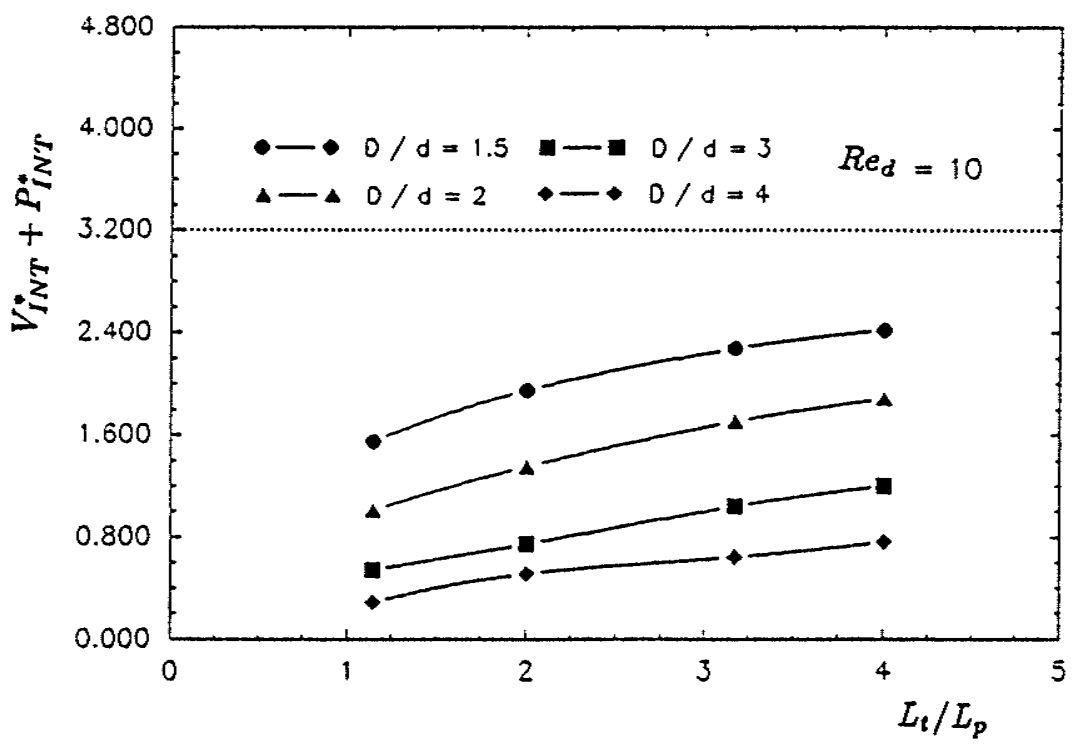

(B). $R e_{d}=10$

Fig. 8.22. Effects of the length ratio $\left(L_{t} / L_{p}\right)$ on the interfacial drag force for $L / d=4.875$. 


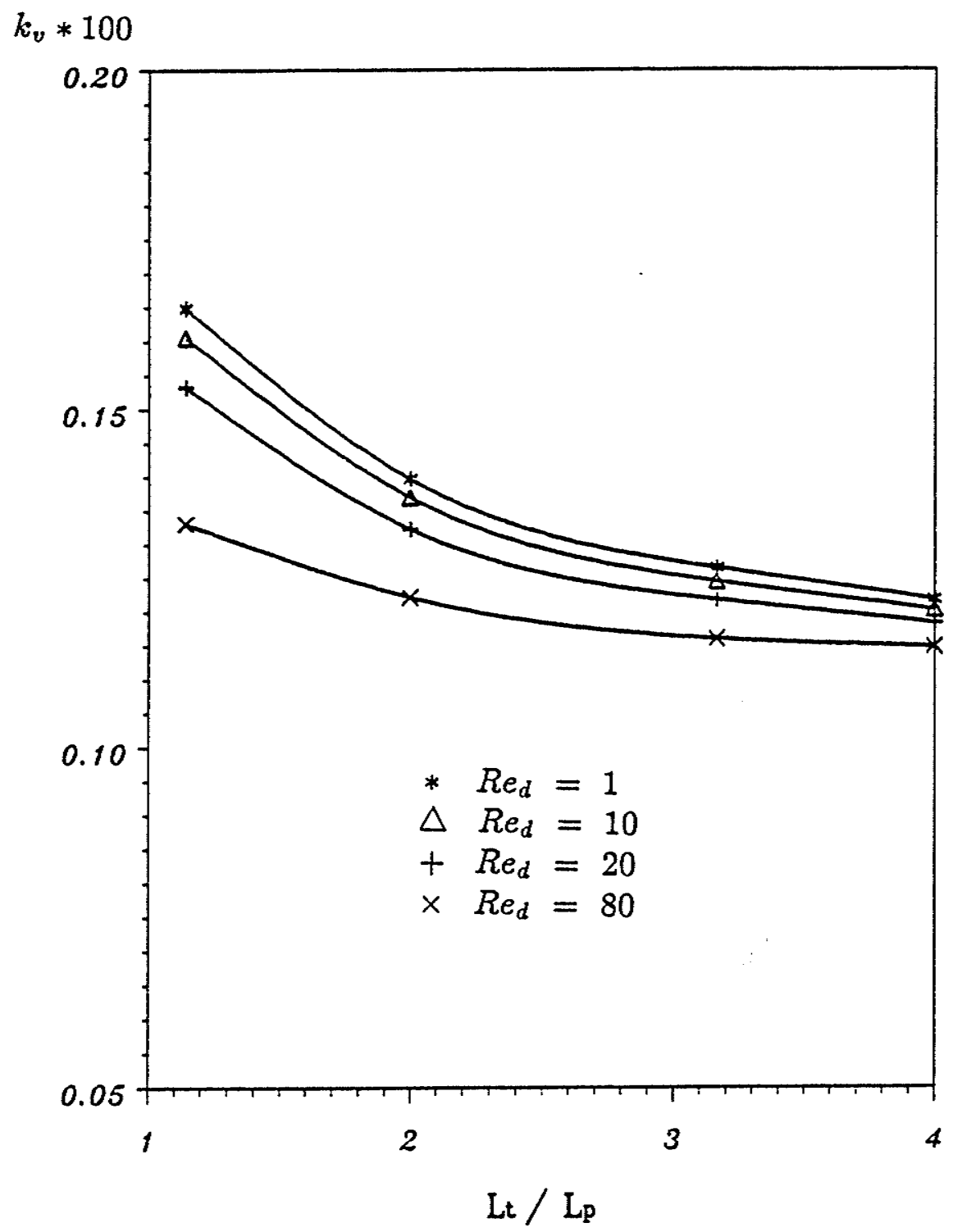

Fig. 8.23. Geometric effects on velocity-dependent permeability for $D / d=4, L / d=4.875$ and $A_{b}^{*}=4.5 * 4.5$. 


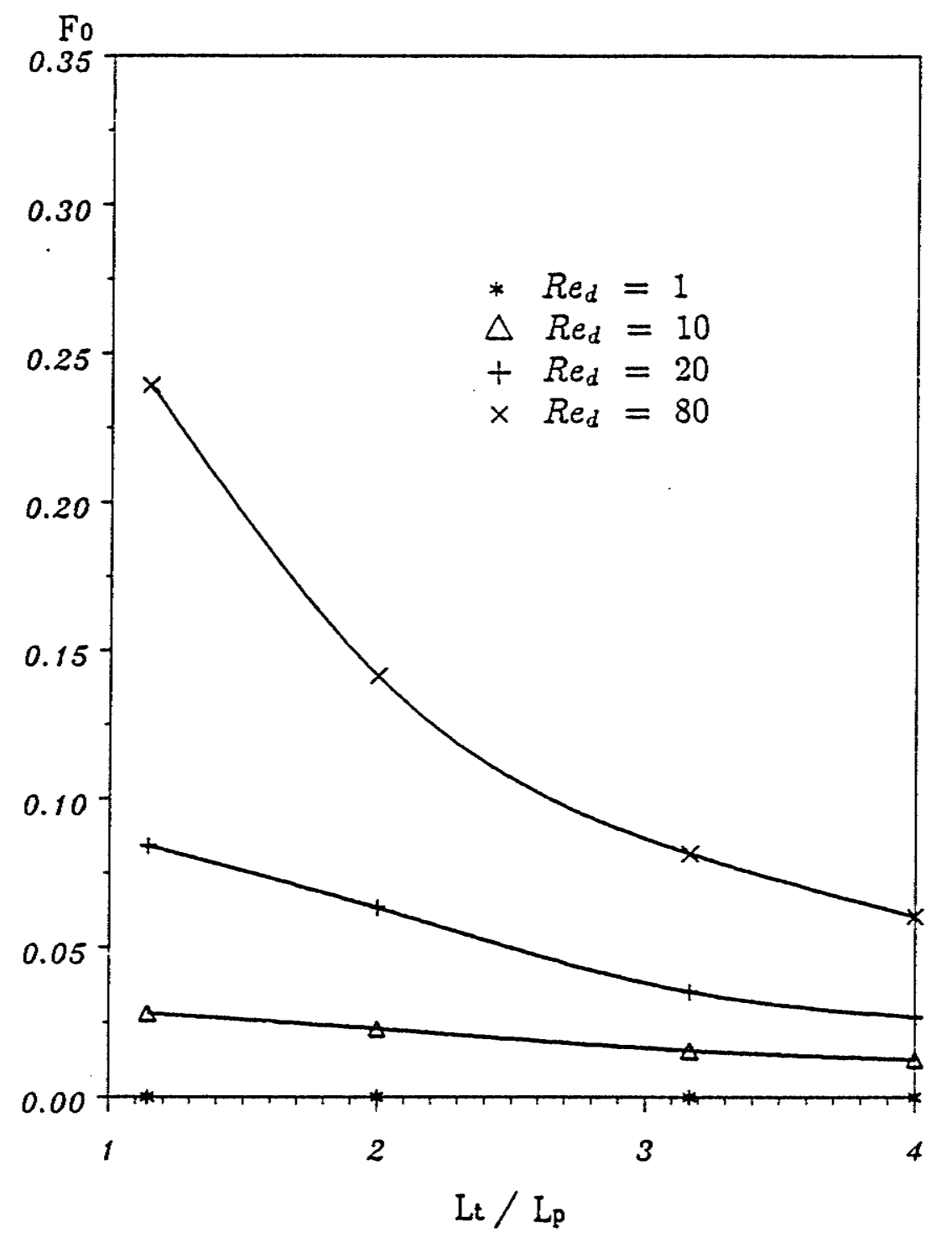

Fig. 8.24. Geometric effects on the Forchheimer number for $D / d=3$ and $L / d=4.875$. 


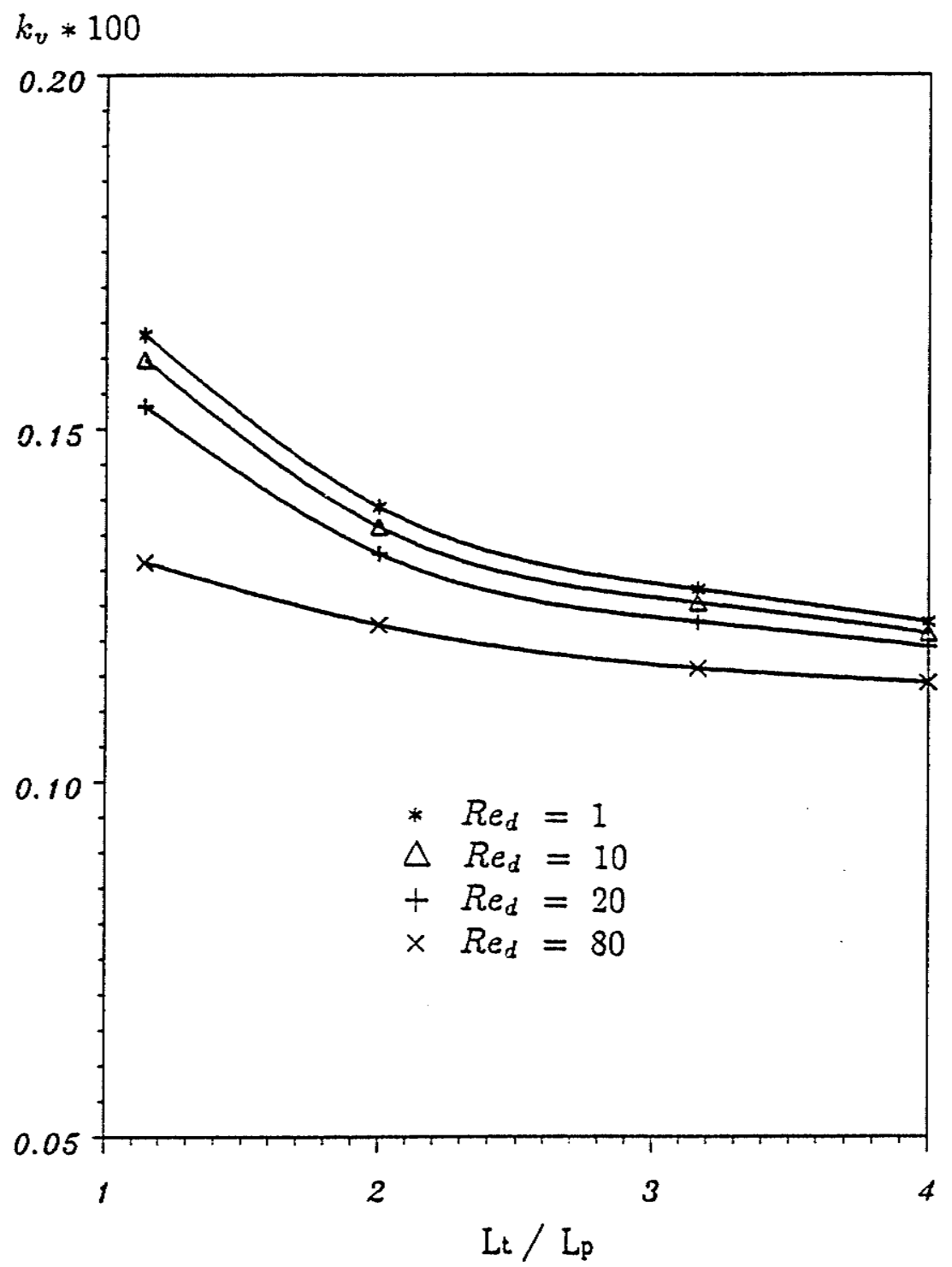

Fig. 8.25. Geometric effects on velocity-dependent permeability for $D / d=2, L / d=4.875$ and $A_{b}^{*}=4.5 * 4.5$. 


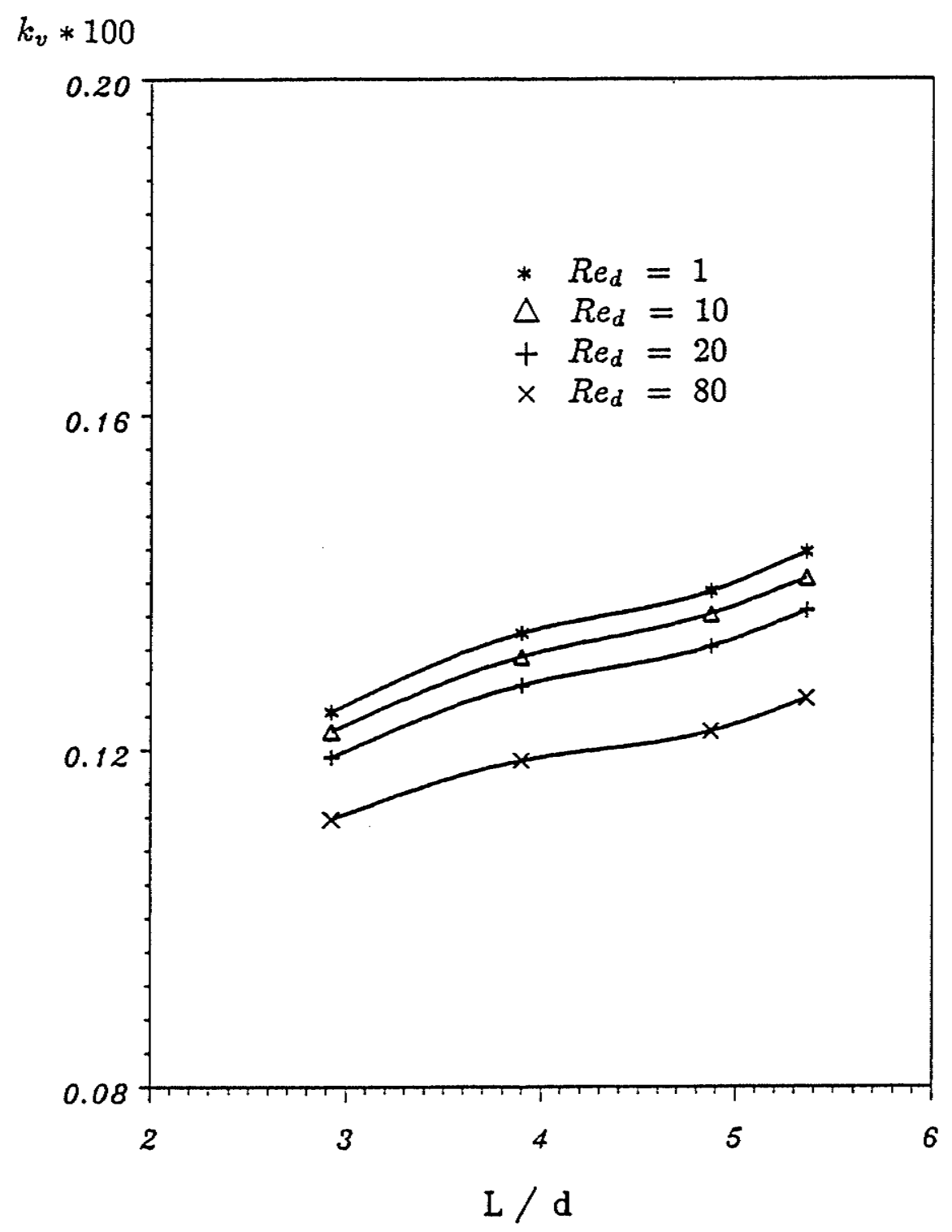

Fig. 8.26. Effects of the length to diameter ratio on velocity-dependent permeability for $D / d=2$ and $L_{t} / L_{p}=2$. 


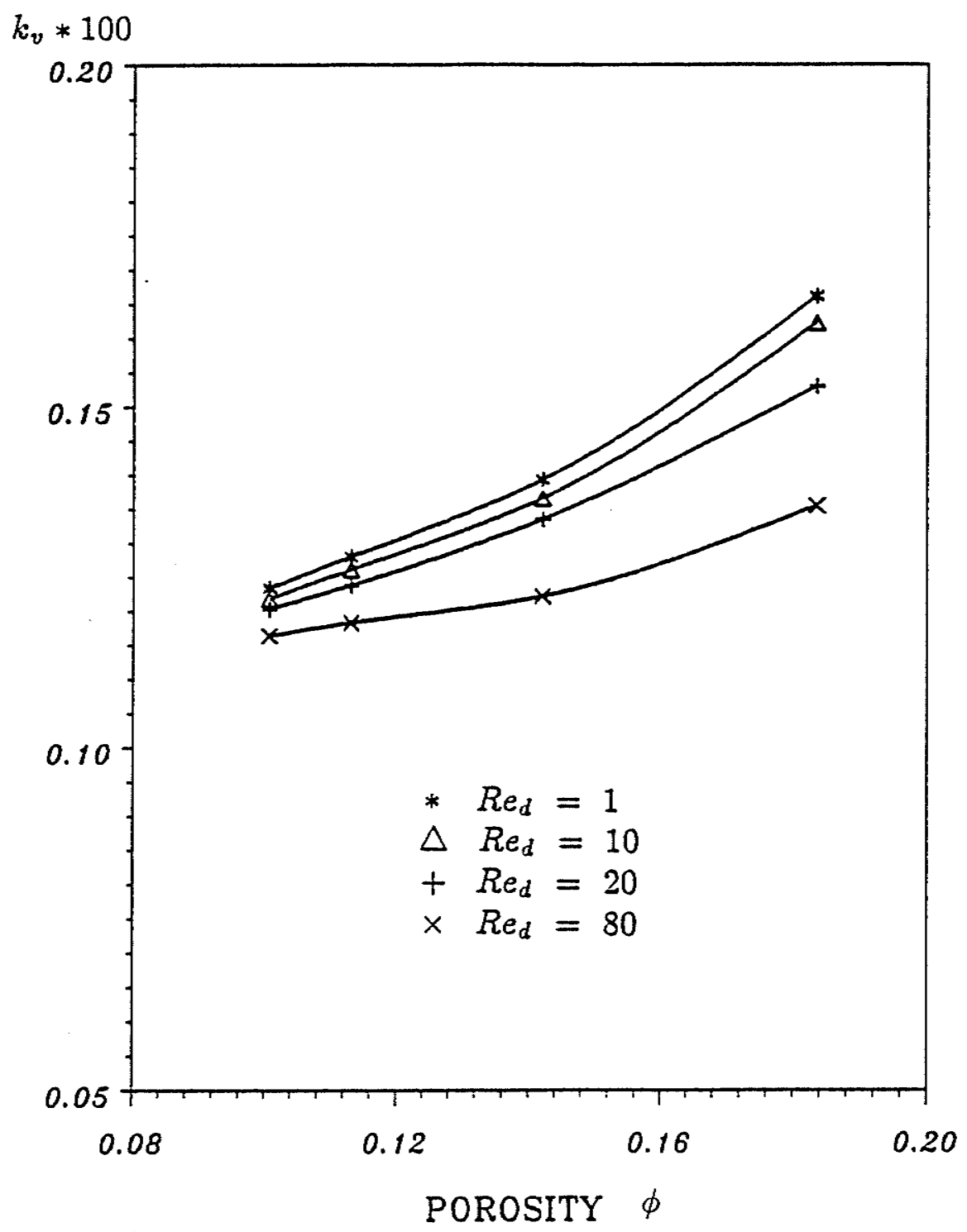

Fig. 8.27. Porosity effects on velocity-dependent permeability for $D / d=2, L / d=4.875$ and $A_{b}^{*}=4.5 * 4.5$. 


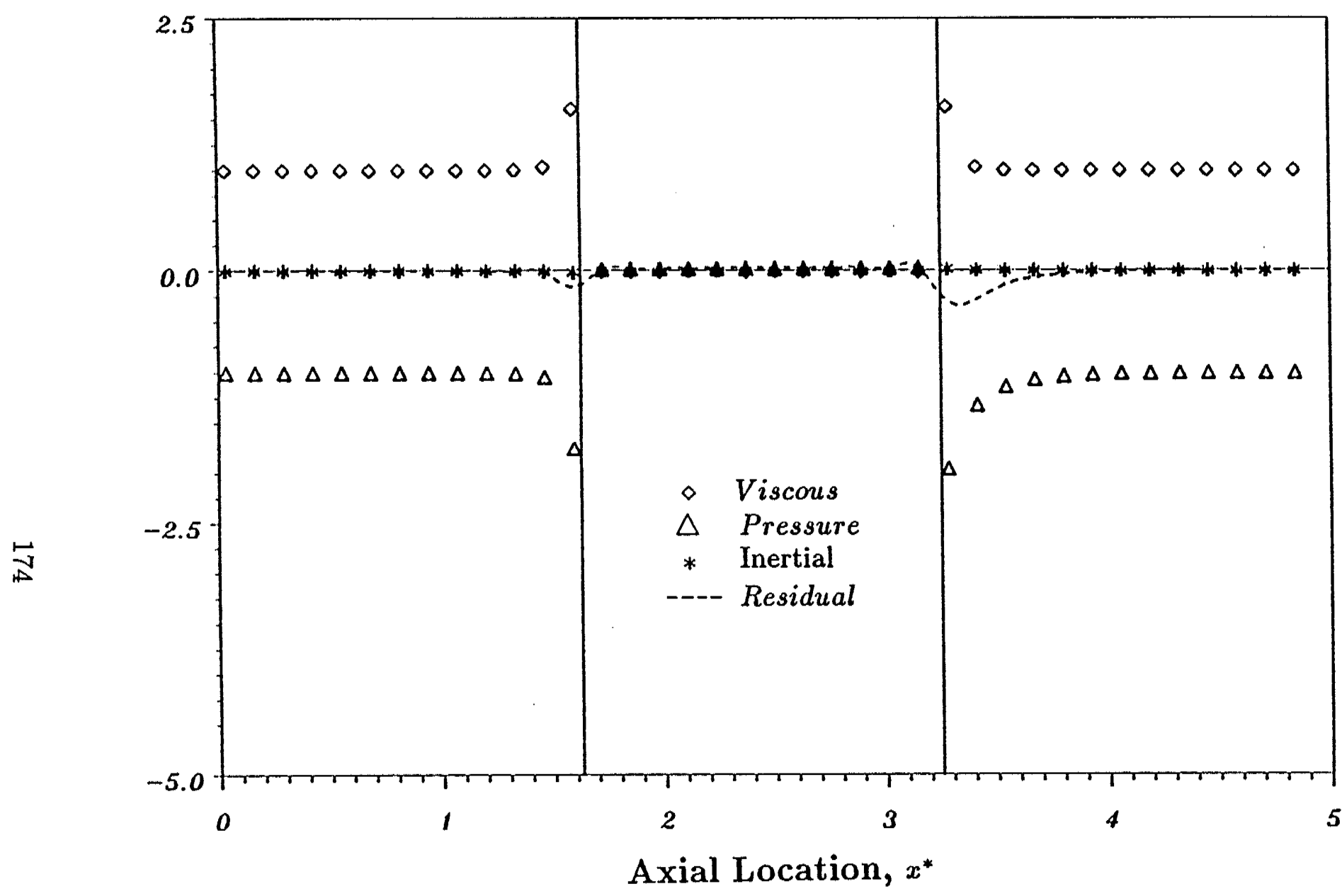

(A). $R e_{d}=0.5$

Fig. 8.28. Balance of the forces along the capillary for $D / d=2, L_{t} / L_{p}=2$ and $L / d=4.875$. 


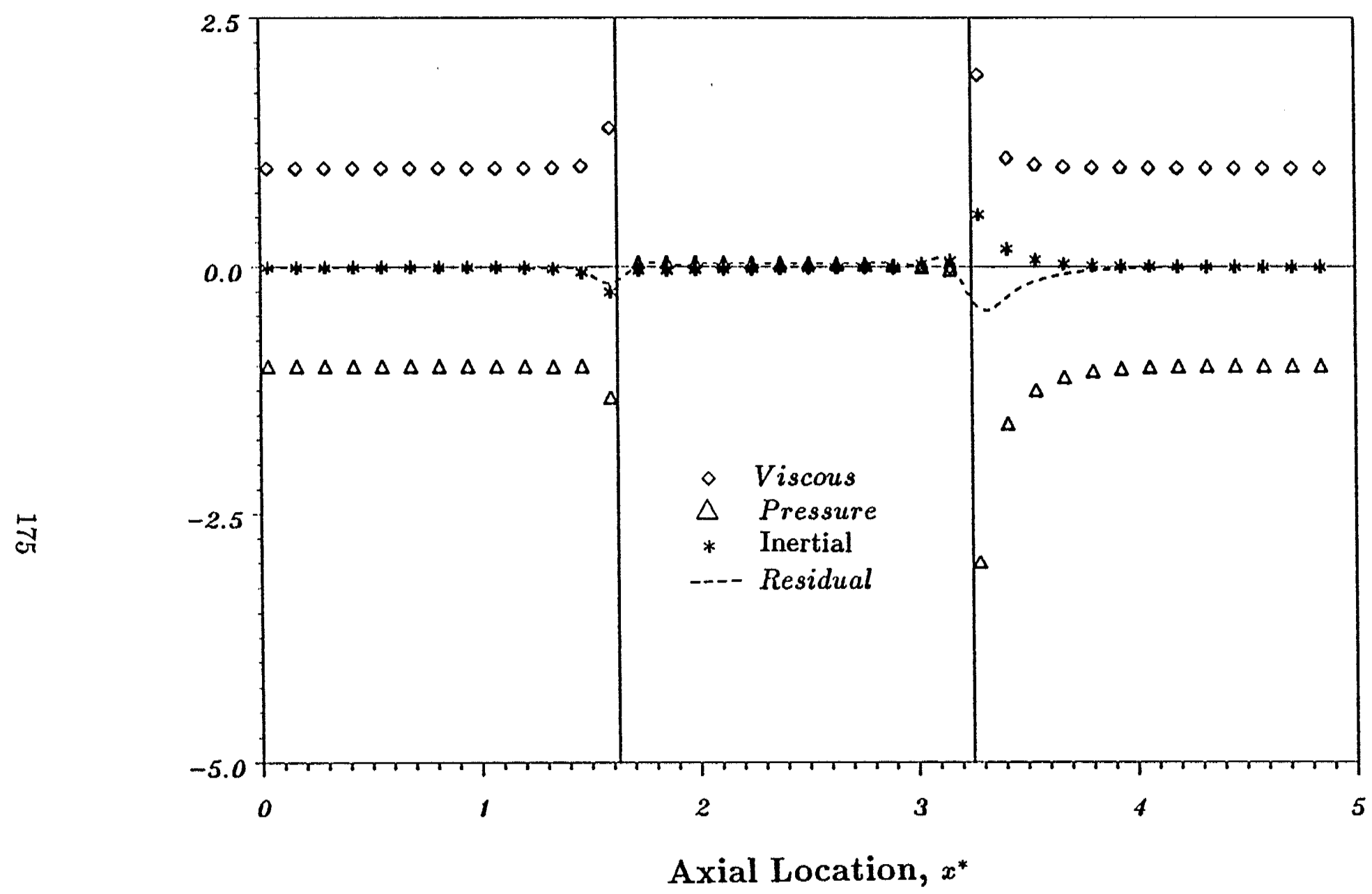

(B). $R e_{d}=13$

Fig. 8.28. Balance of the forces along the capillary for $D / d=2, L_{t} / L_{p}=2$ and $L / d=4.875$. 


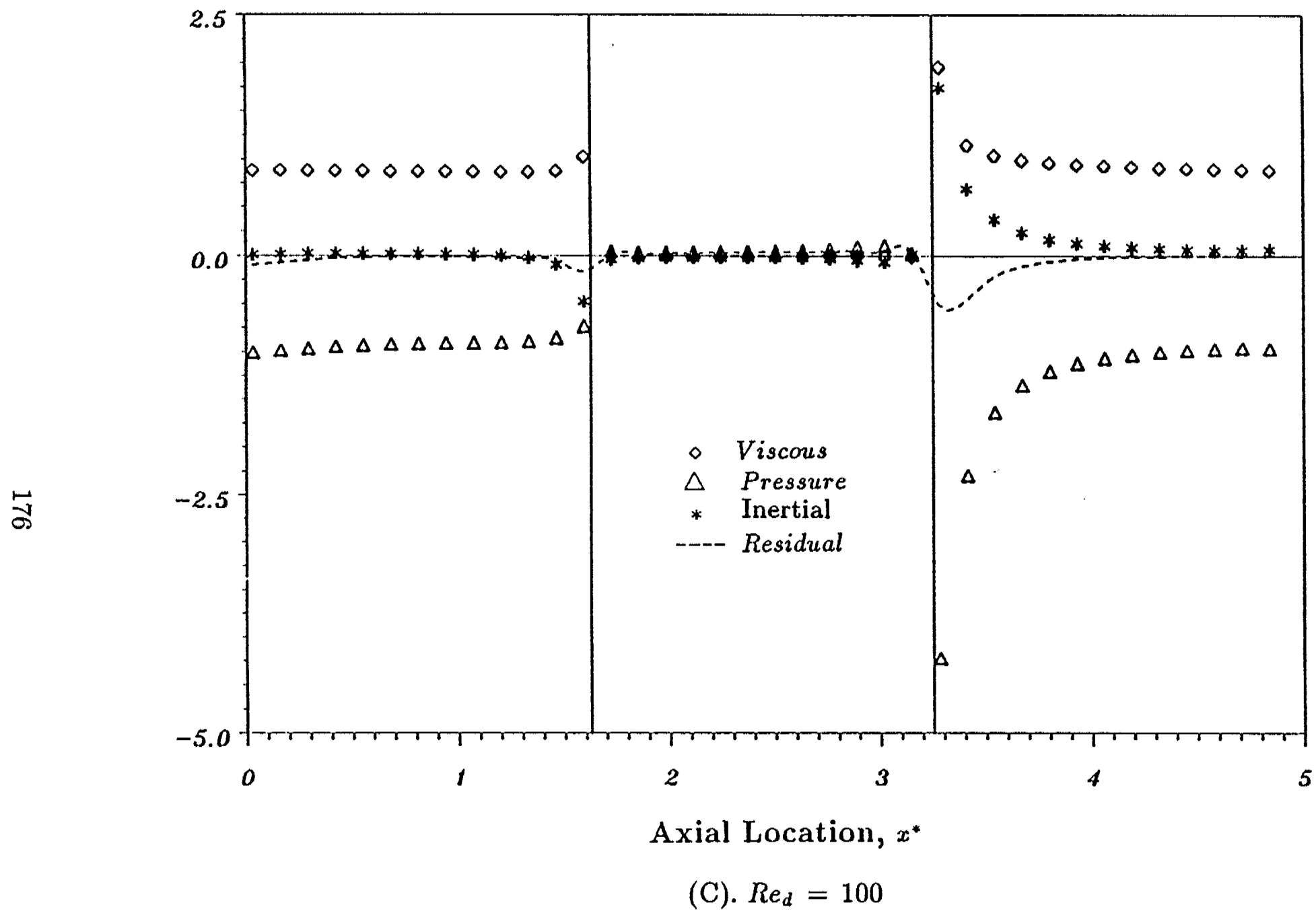

Fig. 8.28. Balance of the forces along the capillary for $D / d=2, L_{t} / L_{p}=2$ and $L / d=4.875$. 


\section{CHAPTER 9}

\section{CONCLUSIONS AND FUTURE}

WORK

This chapter will summarize the accomplishments of the present study on the microscopic analysis of high Forchheimer number flow in porous media by an explicit geometric model. Recommendations for future work are also presented.

\subsection{Accomplishments of the Present Research}

In order to achieve a better understanding and predictions of high $\mathrm{F}$-number flow in porous media, this research has concentrated on the quantitative analysis of the pore level flow and the macroscopic effects of the microscopic phenomena. The problem studied in this dissertation can be divided broadly into two area: (i) the development of the mathematical model for quantitative descriptions of the transport phenomena; (ii) the development of numerical schemes which provide reliable solutions for the subsequent analysis.

The quantitative relationship between the macroscopic effects and the microscopic phenomena was established by a new form of the Forchheimer equation through physical and mathematical considerations, using the volumetric averaging method. The formulation explicitly correlated the macroscopic coefficients with the microstructure and pore flow variables and clarifies the physical mechanism of high $\mathrm{F}-$ number flow in porous media. 
As another unique result, in the second area of this research, the numerical method for accurate predictions of microscopic flow is characterized by two new schemes which deal efficiently with the vorticity singularity and the pressure calculation. The whole solution procedure for the quantitative analysis of porous media flow has been proved to be efficient and reliable.

The main accomplishments of this work are presented below:

1. A computational method and the corresponding computer program have been developed for the numerical solution of the flow fields at the pore scale. The good agreement between the predicted results and the experimental data supports the applicability of this numerical procedure for domains with sudden geometric changes.

2. The pressure computational scheme developed in this study circumvents the uncertainty of the integration method and the divergence of the traditional differential method. Fast convergence and satisfactory accuracy are obtained.

3. A scheme for treating the vorticity singularity at sharp convex corners has been developed. This new and unified method (the vorticity-circulation method) can be applied at both low and high pore Reynolds numbers and exhibits good agreement with both experimental data and other numerical methods.

4. The microscopic flow mechanisms in the porous media have been systematically studied by using the diverging-converging RUC's of a spatially periodic homogeneous model. The numerical results reveal the development of fluid motion at different pore geometries and Reynolds numbers. The detailed information of the microscopic flow patterns served as a basis for the quantitative study of the phenomena on the macroscopic level. 
5. According to physical and mathematical principles, precise hydrodynamic definitions have been given to the macroscopic coefficients: Darcy's law permeability, inertial coefficient and Forchheimer number.

6. Through the quantitative comparisons of different terms in both the microscopic and the macroscopic momentum equations, the results have clearly shown that the fundamental reason for the onset of nonlinearity should be attributed to the microscopic inertial force which has been manifested in the interfacial drag force.

7. The macroscopic coefficients have been numerically calculated at various combinations of micro-geometry and flow rate (the pore Reynolds number), and graphically correlated with relevant microscopic parameters.

\subsection{Recommendations for Future Work}

As already reviewed in Chapter 2, although some of the advantages of the volumetric averaging method are obvious, this procedure gives rise to unclosed problems for the quantities which represent the effects of the microscopic configuration of interphase boundaries and the actual variations of the state variables within each phase. Even for the volumetric averaging method itself, there are still some unsolved practical problems, such as the justification of the assumptions which have been used in the development of the theory (Subsection 2.3.3) and the quantification of the closure forms (Subsection 2.3.4). In other words, a quantitative analysis of the averaging theory must be performed before pursuing any useful solutions by this approach. Regarding the numerical solution of the macroscopic momentum equations, the author recommends the following for future investigation: 
1. To gain an insight into the rôle of certain geometric parameters of porous media (such as the curvature and branching of flow channels, and the heterogeneity of a medium), a more general model should be introduced in the numerical simulation of pore flow;

2. For a more general geometric model, the information at the microscopic level can be employed to verify: (i) if the average theorem is quantitatively correct; (ii) how the average values are distributed within the REV; (iii) if the gradient of average quantities is negligible in the macroscopic momentum equation in a length dimension of the REV. One direct application of the above comparison is the deletion of nondominant terms in the macroscopic governing equations. Although order of magnitude analyses have been widely used in the study of porous media flow, this quantitative analysis undoubtedly provides a more sound basis for the scale assumptions.

3. The dispersion term, $<\tilde{u}_{i} \tilde{u}_{j}>^{f}$, and the interfacial integral terms in the macroscopic equation will not equal to zero in general. Their relative magnitudes and correlations with the geometric properties and averaged variables can be quantitatively achieved through a systematic numerical experiment. 


\section{APPENDIX A}

\section{AVERAGING OF THE VISCOUS TERM}

According to the averaging rule (3.9), the averaging of the viscous term in Equation (3.13) is

$$
\begin{aligned}
<\frac{\partial^{2} u_{i}}{\partial r_{j} \partial r_{j}}>^{f}= & <\frac{\partial}{\partial r_{j}}\left(\frac{\partial u_{i}}{\partial r_{j}}\right)>^{f} \\
= & \frac{\partial}{\partial X_{j}}\left(<\frac{\partial u_{i}}{\partial r_{j}}>^{f}\right)+\frac{1}{\phi V_{b}} \int_{A_{f}}\left(\frac{\partial u_{i}}{\partial r_{j}}\right) \cos \left(\mathbf{n}, \mathbf{e}_{j}\right) d A+ \\
& +<\frac{\partial u_{i}}{\partial r_{j}}>^{f} \frac{1}{\phi} \frac{\partial \phi}{\partial X_{j}} .
\end{aligned}
$$

The first term on RHS of Equation (A.1) can also be written as

$$
\begin{aligned}
\frac{\partial}{\partial X_{j}}\left(<\frac{\partial u_{i}}{\partial r_{j}}>^{f}\right)= & \frac{\partial}{\partial X_{j}}\left[\frac{\partial<u_{i}>^{f}}{\partial X_{j}}+\frac{1}{\phi V_{b}} \int_{A_{f s}} u_{i} \cos \left(\mathbf{n}, \mathbf{e}_{j}\right) d A+\frac{<u_{i}>^{f}}{\phi} \frac{\partial \phi}{\partial X_{j}}\right] \\
= & \frac{\partial^{2}<u_{i}>^{f}}{\partial X_{j} \partial X_{j}}+\frac{<u_{i}>^{f}}{\phi} \frac{\partial^{2} \phi}{\partial X_{j} \partial X_{j}}+\frac{\partial<u_{i}>^{f}}{\partial X_{j}} \frac{1}{\phi} \frac{\partial \phi}{\partial X_{j}}- \\
& -\frac{<u_{i}>^{f}}{\phi^{2}} \frac{\partial \phi}{\partial X_{j}} \frac{\partial \phi}{\partial X_{j}}
\end{aligned}
$$

The integral term in Equation (A.2) vanishes due to the no-slip condition.

The third term on RHS of Equation (A.1) can be expressed in the form

$$
\begin{aligned}
<\frac{\partial u_{i}}{\partial r_{j}}>^{f} \frac{1}{\phi} \frac{\partial \phi}{\partial X_{j}} & =\left[\frac{\partial<u_{i}>^{f}}{\partial X_{j}}+\frac{1}{\phi V_{b}} \int_{A_{f s}} u_{i} \cos \left(\mathbf{n}, \mathbf{e}_{j}\right) d A+\frac{<u_{i}>^{f}}{\phi} \frac{\partial \phi}{\partial X_{j}}\right] \frac{1}{\phi} \frac{\partial \phi}{\partial X_{j}} \\
& =\left[\frac{\partial<u_{i}>^{f}}{\partial X_{j}}+\frac{<u_{i}>^{f}}{\phi} \frac{\partial \phi}{\partial X_{j}}\right] \frac{1}{\phi} \frac{\partial \phi}{\partial X_{j}}
\end{aligned}
$$


Substituting Equations (A.2) and (A.3) into Equation (A.1), one may finally have

$$
\begin{aligned}
<\frac{\partial^{2} u_{i}}{\partial r_{j} \partial r_{j}}>^{f}= & \frac{\partial^{2}<u_{i}>^{f}}{\partial X_{j} \partial X_{j}}+\frac{1}{\phi V_{b}} \int_{A_{f s}}\left(\frac{\partial u_{i}}{\partial r_{j}}\right) \cos \left(\mathbf{n}, \mathbf{e}_{j}\right) d A \\
& +\frac{<u_{i}>^{f}}{\phi} \frac{\partial^{2} \phi}{\partial X_{j} \partial X_{j}}+2 \frac{\partial<u_{i}>^{f}}{\partial X_{j}} \frac{1}{\phi} \frac{\partial \phi}{\partial X_{j}} .
\end{aligned}
$$




\section{APPENDIX B}

\section{ANALYSIS OF THE DISPERSION TERM}

After averaging the microscopic momentum equation (3.12), a dispersion term, $\frac{\partial<\rho \tilde{u}_{i} \tilde{u}_{j}>^{\prime}}{\partial X_{j}}$, is obtained, where $u_{i}$ denote the fluid velocity. In the following, a derivation will be given for this term to clarify some confusion that exists in the analysis of nonlinear flow.

It is noted that each component of $\rho \tilde{u}_{i} \tilde{u}_{j}$ is continuous in $U_{f}$ and generally has a continuous gradient inside $U_{f}$ and on the boundary of $U_{f}$. By applying the definition of the average and the Gauss's theorem for the fluid phase within $U_{b}$, one may obtain

$$
\begin{gathered}
<\frac{\partial\left(\rho \tilde{u}_{i} \tilde{u}_{j}\right)}{\partial r_{j}}>^{f}=\frac{1}{V_{f}} \int_{U_{f}} \frac{\partial\left(\rho \tilde{u}_{i} \tilde{u}_{j}\right)}{\partial r_{j}} d U \\
\quad=\frac{1}{V_{f}} \int_{A_{f *}}\left(\rho \tilde{u}_{i} \tilde{u}_{j}\right) \cos \left(\mathbf{n}, \mathbf{e}_{j}\right) d A \\
\quad+\frac{1}{V_{f}} \int_{A_{f f}}\left(\rho \tilde{u}_{i} \tilde{u}_{j}\right) \cos \left(\mathbf{n}, \mathbf{e}_{j}\right) d A
\end{gathered}
$$

On the other hand, according to the averaging rule (3.9), the LHS of Equation (B.1) can also be expressed in the form

$$
\begin{aligned}
<\frac{\partial\left(\rho \tilde{u}_{i} \tilde{u}_{j}\right)}{\partial r_{j}}>^{f} & =\frac{\partial<\rho \tilde{u}_{i} \tilde{u}_{j}>^{f}}{\partial X_{j}} \\
& +\frac{1}{V_{f}} \int_{A_{f s}}\left(\rho \tilde{u}_{i} \tilde{u}_{j}\right) \cos \left(\mathbf{n}, \mathbf{e}_{j}\right) d A \\
& +\frac{<\rho \tilde{u}_{i} \tilde{u}_{j}>^{f}}{\phi} \frac{\partial \phi}{\partial X_{j}}
\end{aligned}
$$


The combination of Equations (B.1) and (B.2) gives

$$
\begin{gathered}
\frac{\partial<\rho \tilde{u}_{i} \tilde{u}_{j}>^{f}}{\partial X_{j}}=\frac{1}{V_{f}} \int_{A_{f f}}\left(\rho \tilde{u}_{i} \tilde{u}_{j}\right) \cos \left(\mathbf{n}, \mathbf{e}_{j}\right) d A \\
-\frac{<\rho \tilde{u}_{i} \tilde{u}_{j}>^{f}}{\phi} \frac{\partial \phi}{\partial X_{j}} .
\end{gathered}
$$

From Equation (B.3), it is evident that the dispersion term depends not only on the macroscopic properties, but also on the local flow conditions. In other words, the macroscopic change rate of $\left\langle\rho \tilde{u}_{i} \tilde{u}_{j}>^{f}\right.$ is closely related to the net flux of $\rho \tilde{u}_{i} \tilde{u}_{j}$ through the part of the surface bounding $U_{b}$ that intersects only the fluid phase, per unit volume of the fluid phase.

To be more specific, the relation, $\tilde{u}_{i}=u_{i}-<u_{i}>^{f}$, may be substituted into the integral term of Equation (B.3):

$$
\begin{gathered}
\frac{\partial<\rho \tilde{u}_{i} \tilde{u}_{j}>^{f}}{\partial X_{j}}=\frac{\rho}{V_{f}} \int_{A_{f f}} u_{i} u_{j} \cos \left(\mathbf{n}, \mathbf{e}_{j}\right) d A \\
-\frac{<u_{i}>^{f}}{V_{f}} \int_{A_{f f}} \rho u_{j} \cos \left(\mathbf{n}, \mathbf{e}_{j}\right) d A \\
-\frac{\rho<u_{j}>^{f}}{V_{f}} \int_{A_{f f}} u_{i} \cos \left(\mathbf{n}, \mathbf{e}_{j}\right) d A \\
+\frac{\rho<u_{i}>^{f}<u_{j}>^{f}}{V_{f}} \int_{A_{f f}} \cos \left(\mathbf{n}, \mathbf{e}_{j}\right) d A \\
-\frac{<\rho \tilde{u}_{i} \tilde{u}_{j}>^{f}}{\phi} \frac{\partial \phi}{\partial X_{j}} .
\end{gathered}
$$

For simplicity, the fluid phase has been assumed incompressible in the derivation of Equation (B.4). Physically, the first term represents the net momentum flux of unit volume of the fluid phase inside of $U_{b}$ through $A_{f f}$ due to the microscopic movement. The second integral represents the net mass flow rate over the boundary of $U_{b}$. For steady flow, it equals to zero. The third term represents the momentum flux of the microscopic flow due to the average movement. The fourth integral of Equation (B.4) is the projective areas of $A_{f f}$ on the $X_{i}$ directions, and the 
whole term represents the momentum flux of the average movement. The last term is associated with the heterogeneity of the porous media. For a homogeneous medium, this term equals to zero.

It is interesting to reduce the general expression (B.4) into a special form for the case of macroscopically one-dimensional flow through a homogeneous medium:

$$
\begin{gathered}
\frac{d<\rho \tilde{u}_{i} \tilde{u}_{1}>^{f}}{d X}=\frac{\rho}{V_{f}} \int_{A_{f f}} u_{i} u_{j} \cos \left(\mathbf{n}, \mathbf{e}_{j}\right) d A \\
-\frac{\rho<u_{1}>^{f}}{V_{f}} \int_{A_{f f}} u_{i} \cos \left(\mathbf{n}, \mathbf{e}_{1}\right) d A \\
+\frac{\rho<u_{i}>^{f}<u_{1}>^{f}}{V_{f}} \int_{A_{f f}} \cos \left(\mathbf{n}, \mathbf{e}_{1}\right) d A
\end{gathered}
$$

where $u_{1}$ is the component of the microscopic fluid velocity in the $\mathbf{e}_{1}$ direction; $X$ is the only macroscopic coordinate in the present case and is oriented in the direction of $\mathbf{e}_{1}$. For an uniform average flow through a homogeneous medium, the integrals in Equation (B.5) may not necessarily be equal to zero, thus the dispersion term arises. 


\section{APPENDIX C}

\section{DERIVATION OF THE PRESSURE TERM}

By applying the definition of the average and the Gauss's theorem for the fluid phase, one may have

$$
\begin{aligned}
<\frac{\partial p}{\partial r_{i}}>^{f} & =\frac{1}{\phi V_{b}} \int_{V_{f}}\left(\frac{\partial p}{\partial r_{i}}\right) d V \\
& =\frac{1}{\phi V_{b}} \int_{A_{f s}} p \cos \left(\mathbf{n}, \mathbf{e}_{i}\right) d A+\frac{1}{\phi V_{b}} \int_{A_{f f}} p \cos \left(\mathbf{n}, \mathbf{e}_{i}\right) d A
\end{aligned}
$$

On the other hand, according to the averaging rule (3.9), the LHS of Equation (C.1) can also be expressed in the form

$$
<\frac{\partial p}{\partial r_{i}}>^{f}=\frac{\partial<p>^{f}}{\partial X_{i}}+\frac{1}{\phi V_{b}} \int_{A_{f s}} p \cos \left(\mathbf{n}, \mathbf{e}_{i}\right) d A+\frac{<p>^{f}}{\phi} \frac{\partial \phi}{\partial X_{i}}
$$

The combination of Equations (C.1) and (C.2) gives

$$
\frac{\partial<p>^{f}}{\partial X_{i}}=\frac{1}{\phi V_{b}} \int_{A_{f f}} p \cos \left(\mathbf{n}, \mathbf{e}_{i}\right) d A-\frac{<p>^{f}}{\phi} \frac{\partial \phi}{\partial X_{i}}
$$

For the periodic diverging-converging RUC in Figure 3.3, Equation (C.3) becomes

$$
\begin{aligned}
\frac{d<p>^{f}}{\partial X} & =\frac{1}{\phi V_{b}} \int_{A_{f f}} p \cos \left(\mathbf{n}, \mathbf{e}_{i}\right) d A \\
& =\frac{\xi}{\phi L}\left[\frac{1}{A_{f}} \int_{A_{f f}} p \cos \left(\mathbf{n}, \mathbf{e}_{i}\right) d A\right] \\
& =\frac{\xi}{\phi}\left(\frac{\bar{P}_{e x}-\bar{P}_{e n}}{L}\right)
\end{aligned}
$$

where $\bar{P}_{e x}$ and $\bar{P}_{e n}$ are the mean pressures on the exit and entrance, respectively. 


\section{APPENDIX D}

\section{THE ITERATIVE COEFFICIENTS}

The corresponding coefficients $c_{1}, c_{2}, c_{3}, c_{4}, c_{u}$ and source term $s_{i, j}$ in the successive substitution formula (5.16) for each of the variables, $\Omega^{*}, \psi^{*}$ and $p^{*}$.

$\Omega^{*}-$ Equation

$$
\begin{aligned}
c_{1}= & \frac{1}{\Delta x_{i+1, j}^{*}\left(\Delta x_{i, j}^{*}+\Delta x_{i+1, j}^{*}\right)}\left[\frac{2}{G_{i}}+\left(R e_{d} u^{*}\right)_{i, j} \Delta x_{i, j}^{*}\right], \\
c_{2}= & \frac{1}{\Delta x_{i, j}^{*}\left(\Delta x_{i, j}^{*}+\Delta x_{i+1, j}^{*}\right)}\left[\frac{2}{G_{i}}+\left(R e_{d} u^{*}\right)_{i, j} \Delta x_{i+1, j}^{*}\right], \\
c_{3}= & \frac{1}{\Delta r_{i, j+1}^{*}\left(\Delta r_{i, j}^{*}+\Delta r_{i, j+1}^{*}\right)}\left[\frac{2}{G_{j}}+\left(R e_{d} v^{*}-\frac{1}{r^{*}}\right)_{i, j} \Delta r_{i, j}^{*}\right], \\
c_{4}= & \frac{1}{\Delta r_{i, j}^{*}\left(\Delta r_{i, j}^{*}+\Delta r_{i, j+1}^{*}\right)}\left[\frac{2}{G_{j}}+\left(R e_{d} v^{*}-\frac{1}{r^{*}}\right)_{i, j} \Delta r_{i, j+1}^{*}\right], \\
s_{i, j}= & 0, \\
c_{u}= & \frac{1}{\Delta x_{i, j}^{*} \Delta x_{i+1, j}^{*}}\left[\frac{2}{G_{i}}+\left(R e_{d} u^{*}\right)_{i, j}\left(\Delta x_{i+1, j}^{*}-\Delta x_{i, j}^{*}\right)\right]+ \\
& +\frac{1}{\Delta r_{i, j}^{*} \Delta r_{i, j+1}^{*}}\left[\frac{2}{G_{j}}+\left(R e_{d} v^{*}-\frac{1}{r^{*}}\right)_{i, j}\left(\Delta r_{i, j+1}^{*}-\Delta r_{i, j}^{*}\right)\right]- \\
& -\left(\frac{R e_{d} v^{*}}{r^{*}}-\frac{1}{r^{* 2}}\right)_{i, j}
\end{aligned}
$$




\section{$\psi^{*}-$ Equation}

$$
\begin{aligned}
& c_{1}=\frac{2}{\Delta x_{i+1, j}^{*}\left(\Delta x_{i, j}^{*}+\Delta x_{i+1, j}^{*}\right)}, \\
& c_{2}=\frac{2}{\Delta x_{i, j}^{*}\left(\Delta x_{i, j}^{*}+\Delta x_{i+1, j}^{*}\right)}, \\
& c_{3}=\frac{1}{\Delta r_{i, j+1}^{*}\left(\Delta r_{i, j}^{*}+\Delta r_{i, j+1}^{*}\right)}\left[2-\frac{\Delta r_{i, j}^{*}}{r_{i, j}^{*}}\right], \\
& c_{4}=\frac{1}{\Delta r_{i, j}^{*}\left(\Delta r_{i, j}^{*}+\Delta r_{i, j+1}^{*}\right)}\left[2+\frac{\Delta r_{i, j+1}^{*}}{r_{i, j}^{*}}\right], \\
& s_{i, j}=r_{i, j}^{*} \Omega_{i, j}^{*}, \\
& c_{u}=\frac{2}{\Delta x_{i, j}^{*} \Delta x_{i+1, j}^{*}}+\frac{1}{\Delta r_{i, j}^{*} \Delta r_{i, j+1}^{*}}\left[2+\frac{\Delta r_{i, j+1}^{*}-\Delta r_{i, j}^{*}}{r_{i, j}^{*}}\right] .
\end{aligned}
$$

$p^{*}-$ Equation

$$
\begin{aligned}
& c_{1}=\frac{2}{\Delta x_{i+1, j}^{*}\left(\Delta x_{i, j}^{*}+\Delta x_{i+1, j}^{*}\right)}, \\
& c_{2}=\frac{2}{\Delta x_{i, j}^{*}\left(\Delta x_{i, j}^{*}+\Delta x_{i+1, j}^{*}\right)}, \\
& c_{3}=\frac{1}{\Delta r_{i, j+1}^{*}\left(\Delta r_{i, j}^{*}+\Delta r_{i, j+1}^{*}\right)}\left[2-\frac{\Delta r_{i, j}^{*}}{r_{i, j}^{*}}\right], \\
& c_{4}=\frac{1}{\Delta r_{i, j}^{*}\left(\Delta r_{i, j}^{*}+\Delta r_{i, j+1}^{*}\right)}\left[2+\frac{\Delta r_{i, j+1}^{*}}{r_{i, j}^{*}}\right], \\
& s_{i, j}=-2\left[\left(V_{x}\right)_{i, j}\left(U_{r}\right)_{i, j}-\left(U_{x}\right)_{i, j}\left(V_{r}\right)_{i, j}+\frac{v_{i, j}^{*}}{r_{i, j}^{*}}\right], \\
& c_{u}=\frac{2}{\Delta x_{i, j}^{*} \Delta x_{i+1, j}^{*}}+\frac{1}{\Delta r_{i, j}^{*} \Delta r_{i, j+1}^{*}}\left[2+\frac{\Delta r_{i, j+1}^{*}-\Delta r_{i, j}^{*}}{r_{i, j}^{*}}\right] .
\end{aligned}
$$


where

$$
\begin{aligned}
& \left(V_{x}\right)_{i, j}=\frac{1}{\Delta x_{i, j}^{*}+\Delta x_{i+1, j}^{*}}\left(\frac{\Delta x_{i, j}^{*} v_{i+1, j}^{*}}{\Delta x_{i+1, j}^{*}}-\frac{\Delta x_{i+1, j}^{*} v_{i-1, j}^{*}}{\Delta x_{i, j}^{*}}\right)+\frac{\Delta x_{i+1, j}^{*}-\Delta x_{i, j}^{*}}{\Delta x_{i, j}^{*} \Delta x_{i+1, j}^{*}} v_{i, j}^{*}, \\
& \left(U_{r}\right)_{i, j}=\frac{1}{\Delta r_{i, j}^{*}+\Delta r_{i, j+1}^{*}}\left(\frac{\Delta r_{i, j}^{*} u_{i+1, j}^{*}}{\Delta r_{i, j+1}^{*}}-\frac{\Delta r_{i, j+1}^{*} u_{i, j-1}^{*}}{\Delta r_{i, j}^{*}}\right)+\frac{\Delta r_{i, j+1}^{*}-\Delta r_{i, j}^{*}}{\Delta r_{i, j}^{*} \Delta r_{i, j+1}^{*}} u_{i, j}^{*}, \\
& \left(V_{r}\right)_{i, j}=\frac{1}{\Delta r_{i, j}^{*}+\Delta r_{i, j+1}^{*}}\left(\frac{\Delta r_{i, j}^{*} v_{i, j+1}^{*}}{\Delta r_{i, j+1}^{*}}-\frac{\Delta r_{i, j+1}^{*} v_{i, j-1}^{*}}{\Delta r_{i, j}^{*}}\right)+\frac{\Delta r_{i, j+1}^{*}-\Delta r_{i, j}^{*}}{\Delta r_{i, j}^{*} \Delta r_{i, j+1}^{*}} v_{i, j}^{*}, \\
& \left(U_{x}\right)_{i, j}=\frac{1}{\Delta x_{i, j}^{*}+\Delta x_{i+1, j}^{*}}\left(\frac{\Delta x_{i, j}^{*} u_{i+1, j}^{*}}{\Delta x_{i+1, j}^{*}}-\frac{\Delta x_{i+1, j}^{*} u_{i-1, j}^{*}}{\Delta x_{i, j}^{*}}\right)+\frac{\Delta x_{i+1, j}^{*}-\Delta x_{i, j}^{*}}{\Delta x_{i, j}^{*} \Delta x_{i+1, j}^{*}} u_{i, j}^{*},
\end{aligned}
$$




\section{APPENDIX E \\ UNCERTAINTY IN REYNOLDS NUMBER}

The computing equation for Reynolds number is

$$
R e_{d}=\frac{\rho \bar{U}_{d} d}{\mu}=\frac{4 \dot{m}}{\pi \mu d}
$$

where $\dot{m}$ is the mass flow rate through the pipe. Error estimates for measured quantities in Equation (E.1) are:

$$
\begin{array}{ll}
\text { diameter of the pipe: } \quad \frac{\Delta d}{d}= \pm 0.04, \quad(20 \text { to } 1) \\
\text { mass flow rate: } \quad \frac{\Delta \dot{m}}{\dot{m}}= \pm 0.0126, \quad(20 \text { to } 1) \\
\text { dynamic viscosity: } \quad \frac{\Delta \mu}{\mu}= \pm 0.0068 . \quad(20 \text { to } 1)
\end{array}
$$

The overall uncertainty in determining Reynolds number may be estimated by

$$
\begin{aligned}
\frac{\Delta R e_{d}}{R e_{d}} & = \pm\left[\left(\frac{\dot{m}}{R e_{d}} \frac{\partial R e_{d}}{\partial \dot{m}} \frac{\Delta \dot{m}}{\dot{m}}\right)^{2}+\left(\frac{d}{R e_{d}} \frac{\partial R e_{d}}{\partial d} \frac{\Delta d}{d}\right)^{2}+\left(\frac{\mu}{R e_{d}} \frac{\partial R e_{d}}{\partial \mu} \frac{\Delta \mu}{\mu}\right)^{2}\right]^{1 / 2} \\
& = \pm\left[( \pm 0.0126)^{2}+( \pm 0.04)^{2}+( \pm 0.0068)^{2}\right]^{1 / 2}= \pm 0.042 \quad(20 \text { to } 1)
\end{aligned}
$$




\section{REFERENCES}

Anderson, D. A., Tannehill, J. C. and Pletcher, R. H., 1984, Computational Fluid Mechanics and Heat Transfer. Hemisphere, New York.

Astarita, G. and Greco, G., 1968, "Excess pressure drop in laminar flow through a sudden contraction". I+EC Fundamentals, 7(1), PP.27-31.

Azzam, M. I. S. and Dullien, F. A. L., 1977, "Flow in tubes with periodic step changes in diameter: a numerical solution". Chem. Eng. Sci., 32, PP.1445-1455.

Bachmat, Y. and Bear, J., 1986, "Macroscopic modelling of transport phenomena in porous media. 1: The continuum approach". Tranport in Porous Media, 1, PP.213-240.

Barak, A. Z., 1987, "Comments on 'High velocity flow in porous media' by Hassanizadeh and Gray". Transport in Porous Media, 2, PP.533-535.

Barak, A. Z. and Bear, J., 1981, "Flow at high Reynolds number through anisotropic porous media". Adv. Water Resour., 4, PP.54-66.

Bear, J., 1972, Dynamics of Fluid in Porous Media. Elsevier, New York.

Bear, J. and Bachmat, Y., 1986, "Macroscopic modelling of transport phenomena in porous media. 2: Applications to mass, momentum and energy transport". Transport in Porous Media, 1, PP.241-269.

Bear, J. and Bachmat, Y., 1991, Introduction to Modeling of Transport Phenomena in Porous Media. Kluwer Academic Publishers, Dordrecht, The Netherlands.

Blick, E. F., 1966, "Capillary orifice model for high speed flow through porous media". I \& EC, Process Design and Development, No.1, 5, PP.90-94.

Bramley, J. S. and Dennis, S. C. R., 1984, "The numerical solution of two-dimensional flow in a branching channel". Computers and Fluids, 12, PP.339-355. 
Brenner, H., 1980, "Dispersion resulting from flow through spatially periodic porous media". Phil. Trans. R. Soc. Lond. A, 297, PP.81-133.

Brenner, H. and Adler, P. M., 1982, "Dispersion resulting from flow through spatially periodic porous media 2: Surface and intraparticle transport". Phil. Trans. R. Soc. Lond. A, 307, PP.149-200.

Briley, W. R., 1974, "Numerical method for predicting three-dimensional flows in ducts". J. Comp. Phys., 14, 8-28.

Campbell, W. D., and Slattery, J. C., 1963, "Flow in the Entrance of a Tube". J. of Basic Engin., 3, PP.41-46.

Carbonell, R. G. and Whitaker, S., 1984, "Heat and mass transfer in porous media", in Fundamentals of Transport Phenomena in Porous Media (Edited by Bear, J. and Corapcioglu, Y.), Martinus Nijhoff, Brussels.

Carman, P. C., 1937, "Fluid flow through a granular bed". Trans. Inst. Chem. Eng. London, 15, PP.150-156.

Carman, P. C., 1938, "Determination of the specific surface of powders I". J. Soc. Chem. Indus., 57, PP.225-234.

Carman, P. C., 1956, Flow of Gases through Porous Media. Butterworths, London.

Carter, T. R., 1969, Laminar Fluid Flow from a Reservior Up to and Through a Tube-Entrance Region. Ph.D. Thesis, University of Utah, Salt Lake City.

Chien, J, C., 1977a, "A general finite-difference formulation with application to Navier-Stokes equations". Computers and Fluids, 5, PP.15-31.

Chien, J. C., 1977b, "Numerical computation of subsonic conical diffuser flow with nonuniform turbulent inlet conditions". $A E D C-T R-77-73$.

Christiansen, E. B., and Lemmon, H. E., 1965, "Entrance region flow". AIChE J., 9, PP.995-999. 
Collins, M., and Schowalter, W. R., 1963, "Behavior of non-Newtonian fluids in the entry region of a pipe". AIChE J. , 9, PP.804-809.

Coulaud, O., Morel, P. and Caltagirone, J. P., 1988, "Numerical modelling of nonlinear effects in laminar flow through a porous medium". J. Fluid Mech., 190, PP.393-407.

Cvetković, V. D., 1986, "A continuum approach to high velocity flow in porous media". Transport in Porous Media, 1, PP.63-97.

Dagan, G., 1990, Flow and Transport in Porous Formations. Springer-Verlag, New York.

Dagan, G., 1986, "Statistical theory of groundwater flow and transport: pore to laboratory, laboratory to formation, and formation to regional scale". Water Resour. Res., 22(9), PP.120s-134s.

Darcy, H. P. G., 1856, Les fontaines publiques de la ville de Dijon. Victor Dalmont, Paris, 647.

Dennis, S. C. R. and Smith, F. T., 1980, "Steady flow through a channel with a symmetrical contriction in the form of a step". Proc. R. Soc. Lond., A372, PP.393-414.

Dorsey, N. E., 1926, "The Flow of Liquids through Capillaries," Phys. Rev., 26, PP.833-845.

Dullien, F. A. L., 1979, Porous Media Fluid Transport and Pore Stracture. Academic Press, New York.

Dullien, F. A. L. and Azzam, M. I. S., 1973, "Flow rate-pressure gradient measurements in periodically nonuniform capillary tubes". AIChE J., $\mathbf{1 9}$, PP.222-229.

Du Plessis, J. Prieur and Masliyah J. H., 1988, "Mathematical modelling of flow through consolidated isotropic media". Transport in Porous Media, 3, PP.145-161. 
Du Plessis, J. Prieur and Masliyah J. H., 1991, "Flow through isotropic granular porous media". Transport in Porous Media, 6, PP.207-221.

Durst, F. and Loy, T., 1985, "Investigation of laminar flow in a pipe with sudden contractions of cross sectional area". Computers and Fluids, 4, PP.15-36.

Dybbs, A. and Edwards, R. V., 1982, "A new look at porous media fluid mechanicsDarcy to turbulent", in Fundamentals of Transport Phenomena in Porous Media (Edited by Bear, J.), Martinus Nijhoff, Brussels.

Ene, H. I. and Polisevski, D., 1987, Thermal Flow in Porous Media, D. Reidel Publishing Company.

Ergun, S., 1952, "Fluid flow through packed column". Chem. Eng. Prog., 48, PP.89-94.

Forchheimer, P. H., 1901, "Wasserbewegung durch boden". Zeit. Ver. Deutsch. Ing., 45, PP.1782-1788.

Fox, R. W. and McDonald, A. T., 1985, Introduction to Fluid Mechanics. John Wiley and Sons, Toronto.

Gelhar, L. W. and Axness, C. L., 1983, "Three dimensional stochastic analysis of macrodispersion in a quifers". Water Resour. Res., 19(1), PP.161-180.

Ghia, U., Ghia, K. N. and Goyal, R. K., 1979, "Three-dimensional viscous incompressible flow in curved polar ducts". AIAA Paper No. 79-1536.

Gray, W. G., 1975, "A derivation of the equations for multiphase transport". Chem. Eng. Sci., 30, PP.229-233.

Gray, W. G. and O'Neill, K., 1976, "On the general equations for flow in porous media and their reduction to Darcy's law". Water Resour. Res., 12, PP.148-154.

Greenspan, D., 1969, "Numerical studies of steady, viscous, incompressible flow in a channel with a step". J. of Eng. Math., 3, PP.21-28. 
Gupta, M. M., Manohar, R. P. and Noble, B., 1981, "Nature of viscous flows near sharp corners". Computers and Fluids, 4, PP.379-388.

Hannoura, A. A. and Barends, F. B. J., 1981, "Non-Darcy flow: state of art". Proc. Euromech, 143, Delft, PP.37-51.

Hassanizadeh, S. M. and Gray, W. G., 1979a, "General conservation equations for multiphase system: 1. Averaging procedure". Adv. Water Resour., 2, PP.131-144.

Hassanizadeh, S. M. and Gray, W. G., 1979b, "General conservation equations for multiphase system: 2. mass, momenta, energy and entropy equations". Adv. Water Resour., 2, PP.191-203.

Hassanizadeh, S. M. and Gray, W. G., 1980, "General conservation equations for multiphase system: 3 . constitutive theory for porous media flow". Adv. Water Resour., 3, PP.25-40.

Hassanizadeh, S. M. and Gray, W. G., 1987, "High velocity flow in porous media". Transport in Porous Media, 2, PP.521-531.

Holmes, D. B., 1967, Experimental Studies on Laminar Flows in Duct. Ph.D. Thesis, Technische Hogeschool Delft, Delft.

Holstein, $\mathrm{H}$ and Paddon, D. J., 1982, "A finite difference strategy for re-entrant corner flow", in Numerical Methods for Fluid Dynamics. Academic press, New York, PP.341-357.

Howes, F. A. and Whitaker, S., 1985, "The spatial averaging theorem revisited". Chem. Eng. Sci., 40, PP.1387-1392.

Hung, T. K. and E. O. Macagno, E. O., 1966, "Laminar eddies in a two-dimensional conduit expansion". La Houille Blanche, 21(4), PP.391-400.

Irmay, S., 1958, "On the theoretical derivation of Darcy and Forchheimer formulas". Trans. Amer. Geophys. Union, 4(39), PP.702-707. 
Kline, S. J., and McClintock, F. A., 1953, "Describing uncertainty in single-sample experiments," Mechanical Engineering, 75, PP.3-9.

Kozeny, J., 1927, "ت̈ber kapillare leitung des wassers im boden". Stizungsber. Akad. Wiss. Wien, 136, PP.271-306.

Kuwaguti, M., 1965, "Numerical solution of the Navier-Stokes equations for the flow in a channel with a step". MRC TSR 574, Mathematic Research Center, Madison, Wisconsin.

La Nieve, H. L., and Bogue, D. C., 1968, "Correlation of capillary entrance pressure drops with normal stress data". J. Appl. Polymer Sci., 12, PP.353-372.

Ma, H. and Ruth, D. W., 1991, "The numerical computation of the viscous flow in a sudden contraction". Proc. 13th Canadian Congress of Applied Mechanics, Winnipeg, June 2-6, PP.448-449.

Ma, H. and Ruth, D. W., 1993a, "The numerical prediction of pressure distributions in a sudden contraction". Submitted to J. Compu. Fluid Dynamics for publication.

Ma, H. and Ruth, D. W., 1993b, "A new scheme for vorticity computations near a sharp corner". In press, Computers and Fluids.

Ma, H. and Ruth, D. W. 1993c, "The experimental determination of the excess pressure loss in a sudden contraction". Submitted to J. Fluid Eng. for publication.

Macdonald, I. F., El-Sayed, M. S., Mow, K. and Dullien, F. A. L., 1979, "Flow through porous media-Ergun equation revisted". Ind. Eng. Chem. Fund., 18, PP.199-208.

Mei, C. C. and Auriault, J. L., 1991, "The effect of weak inertia on flow through a porous medium". J. Fluid Mech., 222, PP.647-663.

Miyakota, K., 1962, "Contribution to the numerical weather prediction-compu tation with finite difference". Japanese J. of Geophysics, 3, PP.75-190. 
Minkowycz, W. J., Sparrow, E. M., Schneider, G. E. and Pletcher, R. H., 1988, Handbook of Numerical Heat Transfer, John Wiley and Sons, INC..

Mls, R., 1987, "On the existence of the derivative of the volume average". Transport in Porous Media, 2, PP.615-621.

Moffatt, M. K., 1964, "Viscous and resistive eddies near a sharp corner". J. Fluid Mech., 18, PP.1-18.

Nallasamy, M., 1986, "Numerical solution of separating flow due to an obstruction". Computers and Fluids, 14, PP.59-68.

Patankar, S. V., Liu, C. H. and Sparrow, E. M., 1977, "Fully developed flow and heat transfer in ducts having streamwise-periodic variations of cross-sectional area". Transactions of the ASME, 99, PP.180-186.

Payatakes, A. C., Tien, C. and Turian R. M., 1973, "Part 2: Numerical solution of steady incompressible Newtonian flow through periodically constricted tubes". AIChE J., 19, PP.67-76.

Peric, M., Kessler, R. and Scheuerer, G., 1988, "Comparison of finite-volume numerical methods with staggered and colocated grid". Computers and Fluids, 16, PP.389-403.

Press, W. H., Flannery, B. P., Teukolsky, S. A. and Vetterling, W. T., 1987, Numerical Recipes: The Art of Scientific Computing, Cambridge University Press.

Quintard, M. and Whitaker, S., 1988, "Two-phase flow in heterogeneous porous media 1: the influence of large spatial and temporal gradients". Transport in Porous Media, 5, PP.341-379.

Richardson, L. F., 1910, "The approximate arithmetical solutions by finite difference of physical problems involving differential equations, with an application on the stresses in a masonry dam". Trans. of the Royal Soc. of London, Ser. A, 210, PP.307-357. 
Rieman, W., 1928, "The value of the Hagenbach factor in the determination of viscosity by efflux method". J. Am. Chem. Soc., 50, PP.46-55.

Roache, P. J., 1972, Computational Fluid Dynamics. Hermosa Publisers, N.M..

Roache, P. J. and Mueller, T. J., 1970, "Numerical solutions of laminar separation flow field". AIAA J., 8, PP.530-538.

Ruth, D. W. and Ma, H., 1992, "On the derivation of the Forchheimer equation by means of the averaging theorem". Transport in Porous Media, 7, PP.255-264

Sanchez-Palencia, E., 1980, "Non-homogeneous media and vibration theory". Lecture Notes in Physics, 127, Springer-Verlag.

Scheidegger, A.E., 1960, The Physics of Flow Through Porous Media, Macmillan, New York.

Shapiro, A. M., 1981, Fractured Porous Media: Equation Development and Parameter Identification. Ph.D. thesis, Princeton Univ..

Slattery, J. C., 1967, "Flow of viscoelastic fluids through porous media". AIChE J., 13, PP.1066-1071.

Slattery, J. C., 1969, "Single-phase flow through porous media". Am. Inst. Chem. Eng. J., 15, PP.866-872.

Slattery, J. C., 1981, Momentum, Energy, and Mass Transfer in Continua, Robert E. Krieger Publishing Company, Huntington, New York.

Sparrow, E. M., Lin, S. H., and Lundgren, T. S., 1964, "Flow development in the hydrodynamic entrance region of tubes and ducts". Phys. Fluids, 7, PP.338-347.

Stone, T. and Sawatzky, R, 1990, "Modelling flow in porous media using momentum equation". Proc. of Fundamentals of Fluid Transport in Porous Media, May 14-18, 1990, Arles, France. 
Sylvester, N. D. and Rosen, S. L., 1970, "Laminar flow in the entrance region of a cylindrical tube". AIChE J., 16(6), PP.964-966.

Thom, A. and Apelt, C. J., 1961, Field Computations in Engineering and Physics. C. Van Nostrand Company Ltd..

Veverka, V., 1981, "Theorem for the local average of a gradient revised". Chem. Eng. Sci., 36, PP.833-838.

Vrentas, J. S., Duda, J. L., and Bargeron, K. G., 1966, "Effect of axial diffusion of vorticity on flow development in circular conduit: part 1. numerical solutions". AIChE J., 12, PP.837-844.

Vrentas, J. S., and Duda, J. L., 1973, "Flow of a Newtonian fluid through a sudden contraction". Appl. Sci. Res., 28, PP.241-260.

Webster, M. F., 1982, "A technique to solve incompressible flow equations coupled with a stress equation", in Numerical Methods for Fluid Dynamics, Academic press, New York, PP.528-542.

Weissberg, H. L., 1962, "End correction for slow viscous flow through long tubes". Phys. Fluids, 5, PP.1033-1036.

Whitaker, S., 1967, "Diffusion and dispersion in porous media". AIChE J., 13, PP.420-427.

Whitaker, S., 1969, "Advances in the theory of fluid motion in porous media". Ind. Eng. Chem, 61, PP.14-28.

Whitaker, S., 1986, "Flow in porous media I: a derivation of Darcy's law". Transport in Porous Media, 1, PP.3-25.

Woods, L. C., 1954, "A note on the numerical solution of fourth order differential equations". Aeronautical Quarterly, 5, PP.176-184. 